\title{
Diffusion Processes in Membranes Containing Coexisting Domains \\ Investigated by Fluorescence Correlation Spectroscopy
}

\author{
Dissertation \\ zur Erlangung des Doktorgrades \\ der Mathematisch-Naturwissenschaftlichen Fakultäten \\ der Georg-August-Universität zu Göttingen
}

vorgelegt von

Agnieszka Hac

aus Zawiercie, Polen

Göttingen 2003 
D7

Referent: Prof. Dr. T. Salditt

Korreferent: Prof. Dr. T. Heimburg

Tage der mündlichen Prüfung: 17.12.2003 


\section{Acknowledgement}

I pay special thanks to Prof. Thomas Heimburg for his versatile support, also in difficult periods, and for inspiring discussions. He also suggested many interesting points and perspectives to me and encouraged me to think independently. Furthermore he skilfully revised my thesis.

I am very thankful to Heiko Seeger for very fruitful co-operative work on Monte Carlo simulations, his efforts to clarify my scientific considerations as well as wonderful sense of humour to lighten the work, which in itself I found very interesting.

I am deeply appreciate the help of Dr. Rainer Heintzmnn with his expert assistance and crucial discussions about many experimental, theoretical and computer details.

I learnt much from consultations with Prof. Claus Seidel and Dr. Ralf Kühnemuth, Dr. Enno Schweinberger \& Carl Sandhagen about many experimental and technical problems of FCS.

I would like to thank Dr. Rainer Böckmann and Dr. Helmut Grubmüller for the excellent co-operation and their friendly attitude.

Many thanks to the Mechanical Workshop for construction and development of many parts of this FCS setup thus making it working.

Martin Fechner and the IT \& Electronic Service I am grateful for their help with computer problems.

I thank most kindly Thomas Schlötzer for putting a lot of effort in improving my German, taking care about the social life of our group and helping me a lot with acclimatisation in this foreign country.

I like to thank Matthias Fidorra not only for creating beautiful GUVs but also for being such a nice office mate.

I am thankful to Inge Dreger for lending a helping hand with many bureaucracy problems.

I would just like to say many thanks to co-workers at MPI (Denis, Ivan, Vitaly, Jan, Peter, Vesselka and Coni, Tilmann and others) for a good time working with them, all their help and co-operations.

My friends: Ola, Hanja, Stasia, Magda, Samira, Ovidiu, David, Alistair, Matthias, Ralf, Michael \& Jan, Remi and others provided emotional support to me.

I gratefully acknowledge my parents for their support and for enabling me to study physics.

At last but not least I would like to thank Volkswagen Foundation for its financial support. 



\section{Contents}

1 Introduction 1

2 Theory 11

2.1 Diffusion . . . . . . . . . . . . . . . . . 11

2.1.1 Normal and anomalous diffusion . . . . . . . . . . . . . 12

2.1 .2 Hydrodynamic theories . . . . . . . . . . . . . . . . . 14

2.1 .3 "Free volume" theories . . . . . . . . . . . . . . . 16

2.1 .4 Corral diffusion . . . . . . . . . . . . . . . . . 18

2.2 Fluorescence Correlation Spectroscopy ～. . . . . . . . . . . . . . . . . 19

$2.2 .1 \quad$ FCS Theory . . . . . . . . . . . . . . . . . . . . . 19

2.2 .2 Labels . . . . . . . . . . . . . . . . . . . 25

2.3 Differential Scanning Calorimetry . . . . . . . . . . . . . . . 27

2.3 .1 Phase transition . . . . . . . . . . . . . . . . . . 28

2.3 .2 Theory of the phase diagram $\ldots \ldots \ldots$. . . . . . . . 30

2.3.2.1 The ideal solution theory . . . . . . . . . . 32

2.3.2.2 The regular solution theory . . . . . . . . . 35

2.4 Monte Carlo Simulations . . . . . . . . . . . . . . . . . . . . . 37

2.4.1 Lattice geometry, states and configuration . . . . . . . . . . . 37

2.4.2 Determination of the model parameters . . . . . . . . . . . . 41

2.4 .3 Steps in the Monte Carlo simulations . . . . . . . . . . . . . . . 43

2.4 .4 Simulation of FCS experiments . . . . . . . . . . . 46

3 Material and Methods 49

3.1 Fluorescence Correlation Spectroscopy . . . . . . . . . . . . . . . . . . 49

$3.1 .1 \quad$ FCS Setup . . . . . . . . . . . . . . . . . . . 49

3.1.1.1 Calculations for telescope lenses and pinhole . . . . . . . . 51

3.1 .1 .2 Focus size . . . . . . . . . . . . . . 54 
3.1.1.3 The excitation power of the laser . . . . . . . . . 56

$3.1 .2 \quad$ FCS properties . . . . . . . . . . . . . . . 57

3.2 Membrane preparation ...................... 61

$3.2 .1 \quad$ Label properties . . . . . . . . . . . . . . . . 63

4 Results 69

4.1 Single molecule measurements . . . . . . . . . . . . . . . . 70

4.2 Domain visualisation $\ldots \ldots \ldots \ldots$. . . . . . . . . . . . . . . . . . . .

4.2.1 The phase diagram . . . . . . . . . . . . . . 73

4.2.2 Monte Carlo snapshots . . . . . . . . . . . . . . 78

4.2 .3 Confocal Fluorescence Microscopy . . . . . . . . . . . . . . . 79

4.3 Diffusion in a DLPC:DPPC mixture . . . . . . . . . . . . . . . 81

4.4 Diffusion in a DMPC:DSPC mixture . . . . . . . . . . . . . . 90

$4.4 .1 \quad$ FCS results $\ldots \ldots \ldots \ldots$. . . . . . . . . . . . . . . 90

4.4 .2 Monte Carlo simulations . . . . . . . . . . . . . . . . . 95

4.5 Ionic strength dependence of diffusion $\ldots \ldots \ldots$. . . . . . . . . . 100

4.5.1 Calorimetric measurements . . . . . . . . . . . . . 101

$4.5 .2 \quad$ FCS experiment . . . . . . . . . . . . . . . . 103

4.5.3 Molecular Dynamic simulations . . . . . . . . . . . . . . . 109

$\begin{array}{llr}5 & \text { Discussion and Conclusions } & 115\end{array}$

$\begin{array}{lll}6 & \text { Outlook } & 123\end{array}$

\begin{tabular}{ll}
\hline Abbreviations & 125
\end{tabular}

$\begin{array}{ll}\text { Bibliography } & 138\end{array}$ 


\section{Chapter 1}

\section{Introduction}

The development of biological membranes was an important occurrence in the evolution of life. Every highly evolved cell is full of membranes (see Figure 1.1), since it is comprised of many functional units or organelles. Each of these units also contains one or more membranes.

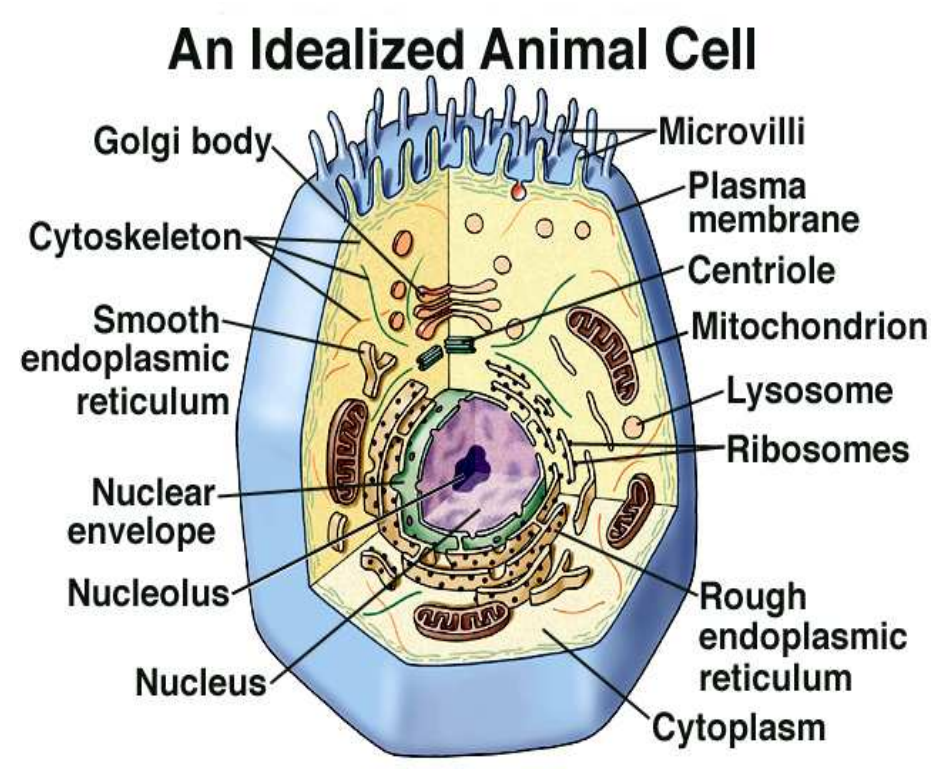

Figure 1.1: The illustration of variety of organelles in animal cell [55].

The cell membrane performs a number of important functions for the cell such as $[6 ; 55$; 95]:

- the separation of the cell from its outside environment, 
- controlling which molecules enter and leave the cell,

- providing the support and working environment for a great variety of enzymes, receptors and antigens and

- recognition of chemical signals.

All of the membranes inside the cell separate compartments to protect important processes and events.

Biological membranes contain an interesting array of molecules $[13 ; 17 ; 55 ; 72 ; 115]$. These include glycerophospholipids, sphingolipids, steroids (e.g., cholesterol), proteins, carbohydrates and others which are conjugates containing molecules from two or more of these groups. The lipid composition varies between different cells and organelles (refer to Table. 1.1). The primary structural requirement for a molecule to reside in a lipid bilayer is its

Table 1.1: The lipid composition in different membranes; those in bold type are the most common lipids [115].

\begin{tabular}{|l|c|c|l|l|}
\hline & \multicolumn{3}{|c|}{ Percentage of total composition in } \\
& $\begin{array}{c}\text { Human } \\
\text { Erythrocyte } \\
\text { Plasma } \\
\text { Lembrane }\end{array}$ & $\begin{array}{c}\text { Human } \\
\text { Myelin }\end{array}$ & $\begin{array}{l}\text { Beef heart } \\
\text { Mitochondria }\end{array}$ & $\begin{array}{l}\text { E. coli } \\
\text { Cell } \\
\text { membrane }\end{array}$ \\
\hline Phosphatidic acid & 1.5 & 0.5 & 0 & 0 \\
\hline Phosphatidylcholine & 19 & 10 & 39 & 0 \\
\hline Phosphatidylethanoloamine & 18 & 20 & 27 & 65 \\
\hline Phosphatidylglycerol & 0 & 0 & 0 & 18 \\
\hline Phosphatidyllinositol & 1 & 1 & 7 & 0 \\
\hline Phosphatidylserine & 8 & 8 & 0.5 & 0 \\
\hline Sphingomyelin & 17.5 & 8.5 & 0 & 0 \\
\hline Glycolipids & 10 & 26 & 0 & 0 \\
\hline Cholesterol & 25 & 26 & 3 & 0 \\
\hline Others & 0 & 0 & 23.5 & 17 \\
\hline
\end{tabular}

amphiphilic nature (i.e., part polar, part nonpolar). Because of this lipids form aggregates in an aqueous environment in which their hydrocarbon region is kept apart from water (see Figure 1.2).

Lipids are stabilised in aggregates by the hydrophobic interaction, van der Waal's interactions between the hydrocarbon chains, electrostatic interactions and hydrogen bonds between 

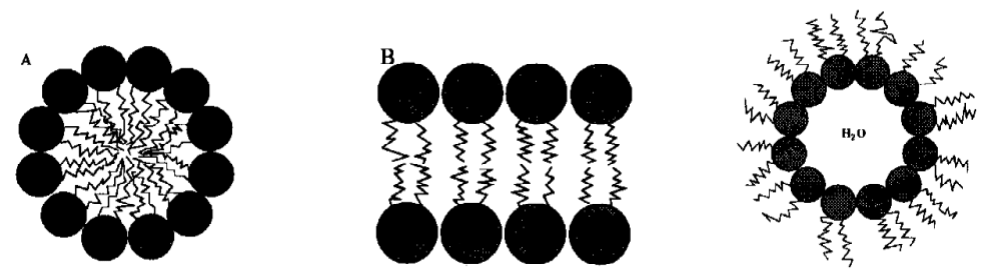

Figure 1.2: Examples of membrane structures: micelle, bilayer and inverse hexagonal phase [55].

the polar heads $[53 ; 55]$. The most relevant structure for the biological membrane is the lipid bilayer. The other molecules, e.g. proteins, are oriented within the lipid bilayer so that their nonpolar part is inserted into the nonpolar core of the bilayer. Similarly, the polar parts are incorporated into the polar headgroups of the lipids. There are extrinsic or peripheral proteins that are attached to the surfaces of membranes, while the other proteins (known as intrinsic or integral) interact with membranes so that their nonpolar units become inserted into the nonpolar part of the bilayer and the remaining polar parts continue to stick out [16; $32 ; 84$; 95; 115]. There are also transmembrane proteins, such as bacteriorhodopsin, that can pass through the lipid bilayer [32].

Phospholipids are one of the most common lipids (refer to Table 11). Most phospholipids are derived from glycerol to which two fatty acyl residues and a single phosphate, together with phosphate esters, are attached [55, 72]. Phospholipids consist of a polar headgroup and two apolar chains. The lipids differ in a chain length and number of unsaturated bonds. Figure 1.3 shows an example of a phospholipid.

The left tail is a straight fatty acid (saturated). The right one has a kink in the tail because of the cis double bond (unsaturated). This kink affects packing and movement in the lateral plane of the membrane.

One important property of a membrane is a melting transition at a characteristic temperature. Figure 1.4 shows the bilayer melting transition.

On the left part of Figure 1.3 and Figure 1.4 the lipid chains are presented in all-trans conformation, where they are parallel and extended fully. This is the order gel state and occurs when lipids are in their state of minimum of energy. With increasing temperature, a free rotation around each $\mathrm{C}-\mathrm{C}$ bond in the hydrocarbon chains occurs, this being responsible for lipid melting. This rotation of $\pm 120^{\circ}$ around the $\mathrm{C}$ - $\mathrm{C}$ bond changes the trans conformation 


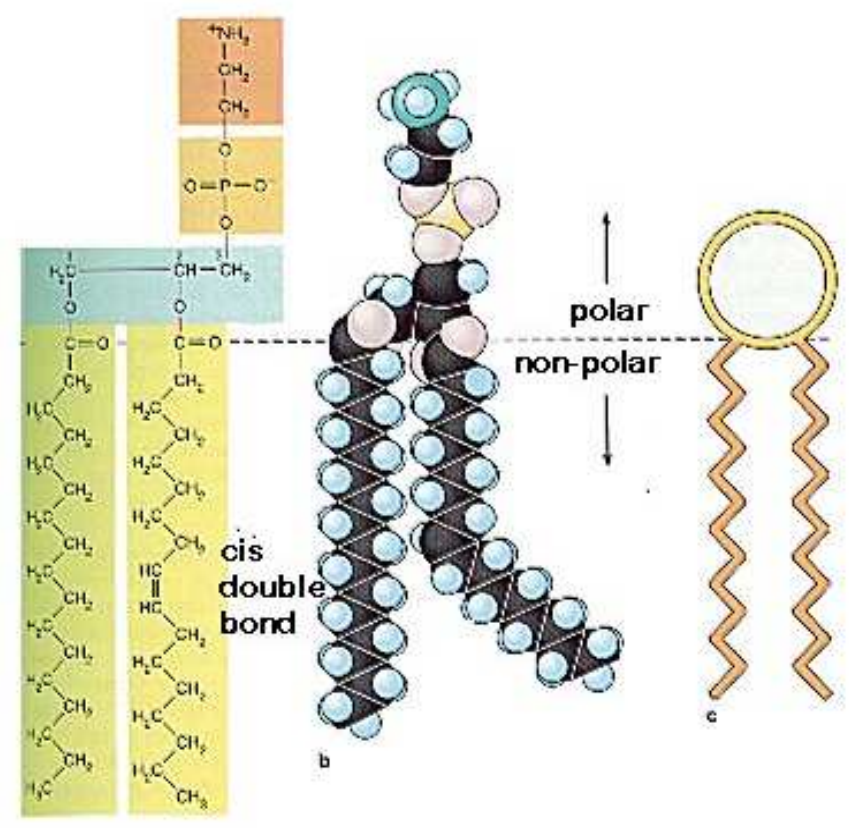

Figure 1.3: The example schema of a phospholipid [55].

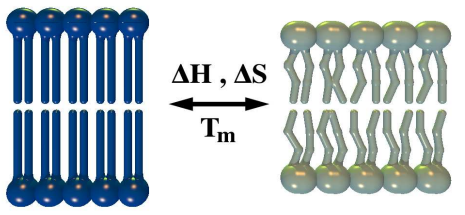

Figure 1.4: The scheme of the melting transition in a lipid bilayer.

into the gauche conformation $[35 ; 55 ; 72 ; 79 ; 89]$. Above the characteristic temperature, lipid chains are in a highly disordered fluid state where their orientation is less parallel. Then they are in any of a great number of excited gauche conformations.

The transition temperature itself depends on the length and saturation of the lipid chains, composition of the polar head group, ionic strength (ions), $\mathrm{pH}$, as well as the presence of other molecules such as proteins or cholesterol $[43 ; 40 ; 101 ; 84]$. Most biological lipids melt at a temperature below the physiological temperature $[16 ; 43]$. Above the melting temperature, lipids exist in a fluid, disordered state and below the melting temperature, in a gel ordered state. In going from the gel to the fluid state, the thickness of the bilayer decreases and the area per molecule increases. In the fluid state, a considerable amount of molecular motion is possible, whereas this is reduced in the gel state due to close molecular packing. Many basic cell processes like growth, division, fusion, secretion, endocytosis, exocytosis 
depend on membrane component movement [55].

The membrane structure is derived on the basis of diffraction analysis (x-rays, neutrons and electrons) [68], electron microscopy [65], Raman spectroscopy [14; 122] and spectroscopic approaches (NMR, EPR) [10; 65; 71]. There are several membrane models. One example is the fluid membrane mosaic model developed by Singer and Nicolson in 1972 [107]. In this, the membrane consists of two layers of lipids with proteins embedded within these layers (see Figure 1.5 (a)). This model takes into account the fluidity of the membrane, but not the variety of lipids and lipid states (gel and fluid) normally present in biological membranes. The "mattress model" proposed by Mouritsen and Bloom is based on a real two-component solution theory which allows for phase separation [82] (see Figure 1.5 (b)). With this model the heterogenities and interactions between proteins and lipids have been studied theoretically and experimentally. The "mattress model" was modified to better suit our needs (see Figure 1.5 (c) [43].

In recent years it has been established that the components of biological membranes (protein and lipids) are not distributed homogeneously within the membrane plane. They form different domains and, e.g., so-called "rafts" [2; 22; 106]. Rafts are microdomains rich in cholesterol, sphingolipids and some specific proteins. Cholesterol itself is a very interesting molecule. At temperatures below the melting temperature of a membrane it increases fluidity by preventing close packing of acyl chains, inhibiting crystallisation and destabilising the gel phase. Above the melting temperature cholesterol causes gel clusters to form and immobilise the surrounding few lipid molecules. More than $30 \mathrm{~mol} \%$ of cholesterol eliminates phase transition [4; 38].

However, even in simple pure lipid systems, macro- and micro-domains can be observed [6; 57; 59]. The formation of domains is related to the so-called "hydrophobic mismatch", which depicts interactions of lipid chains with water when the hydrophobic chains differ in length (see Figure 1.5 b) $[82 ; 81 ; 83]$. It is known that the disordered fluid chains are effectively shorter than the ordered gel chains [68]. Each lipid tends to be surrounded by lipids with similar chain lengths in order to protect the hydrophobic parts from the water.

Probably one of the first studies proposing domain formation in a gel and fluid coexistence phase was carried out by Jain [56]. He proposed the "plate model" of membrane structure with the organised (gel like) and disorganised (fluid like) regions viewed as plates (refer to Figure 1.6 a). 


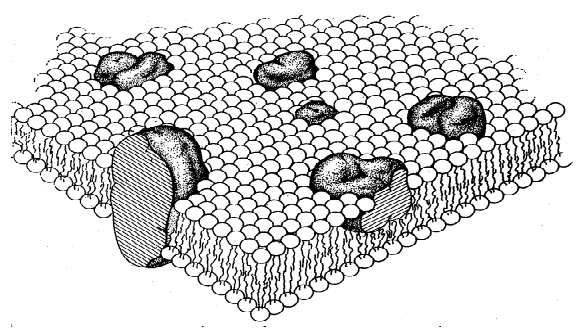

(a)

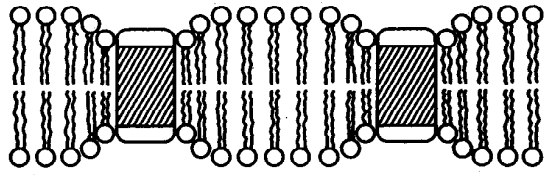

(b)

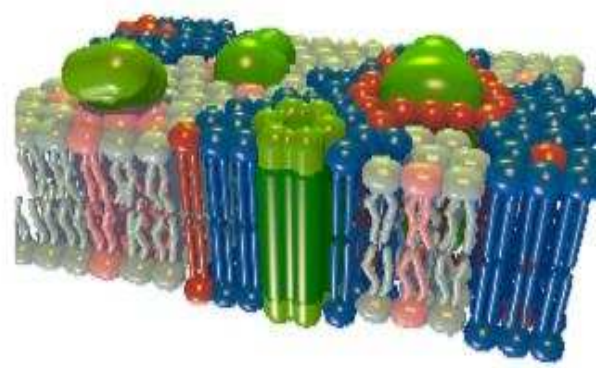

(c)

Figure 1.5: (a). The fluid mosaic model of Singer and Nicolson [107]. (b) The "mattress model" of membrane. Lipids are indicated as a circular polar head and two flexible acyl chains and amphiphilic impurities (proteins) as rod-shaped objects. The illustration of "hydrophobic mismatch" when the impurity is shorter than the lipid bilayer thickness [82]. (c) Schematic drawing of a biological membrane, showing different lipids in different states distributed inhomogeneously within the membrane plane. The dark colours (red and blue) correspond to the gel domains and the light ones to the fluid domains. Proteins (green) penetrate through the membrane or are bound to its surface [43].

The coexistence of gel and fluid domains has recently been visualised by Confocal Fluorescence Microscopy $\left(\mathrm{CFM}^{1} \backslash\right.$ shown in Figure $1.6(\mathrm{~b})[6 ; 22,59]$ and Atomic Force Microscopy $\left(\mathrm{AFM}^{2} \mathrm{~T}\right)$ as shown in Figure 1.ó (c) [42; 3.5]. Membrane heterogenity meanwhile has been detected experimentally in model membranes as well in biological membranes [7; 13. 100].

Theoretical studies based on statistical thermodynamic fluctuations try to describe lipid

\footnotetext{
${ }^{1}$ The principles of CFM are explained in Section 4.2.3.

${ }^{2}$ The sample is rastered with a sharp tip mounted on a cantilever, which moves with a constant force. In this way the sample is scanned, resulting in a topographical map of the scanned region.
} 


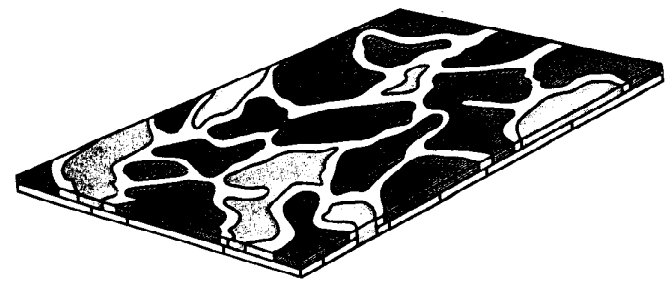

(a)

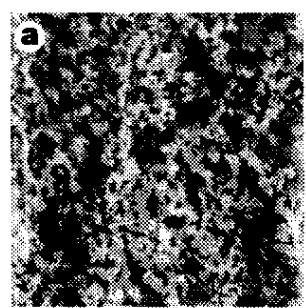

(c)

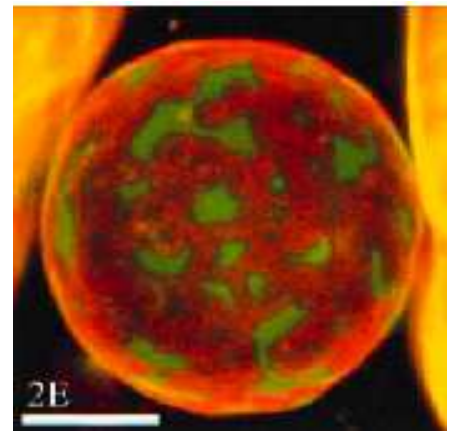

(b)

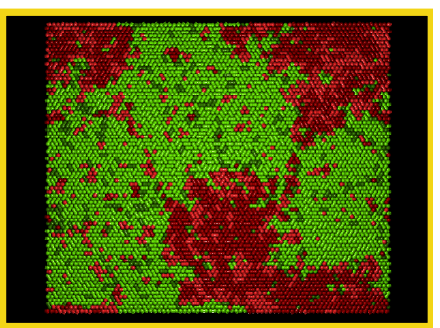

(d)

Figure 1.6: Examples of domains obtained or predicted by different techniques: (a) The "plate model" of membrane structure. The organised and disorganised regions are viewed as plates, each having characteristic system properties specified by its components [56]. (b) Confocal Fluorescence Microscopy image of DLPC:DPPC 20:80 GUV. It shows ordered gel regions in red and fluid phase in green [59]. (c) Atomic Force Microscopy map of DSPC monolayer at its melting temperature. The high difference between light and dark areas is about $0.5 \mathrm{~nm}$ [85]. (d) Monte Carlo snapshoot of a DMPC:DSPC 70:30 mixture at $304 \mathrm{~K}$. The red symbols correspond to the gel domains and the green symbols to the fluid domains.

melting and self-organisation of a multi-component system $[23 ; 12 ; 11 ; 19 ; 53 ; 69 ; 75 ; 81$; 80. 88; 89]. There are various methods of investigating the melting of lipids theoretically, two of these are mean-field theory and Monte Carlo simulations (MC). The mean-field theory model proposed by Marcelja takes average energies and entropies of lipid ensembles into account in calculating the lipid ordering chains [69]. It does not account for phase separation, unlike MC simulations. MC simulations are statistical mechanical lattice models including nearest-neighbour interactions. Usually Monte Carlo simulations can be done on the basis of the 10 -state Pink model $[14 ; 49 ; 81 ; 88 ; 89]$ or of the 2 -state Ising model $[42 ; 44 ; 76 ; 109$; 110; 111]. MC simulations postulate the formation of gel and fluid domains as a consequence of near neighbour interactions as in Figure 1.6 (d)) [42;44; 49; 57]. This is one of the very important predictions originating from the thermodynamical anaiysis of membrane properties. With MC the diffusion processes in membranes can also be simulated. 
The relevance of domain structure and dynamics is discussed highly as a putative control mechanism of diffusion processes in membranes [92] and thus of signalling cascades [13; 106]. In recent years the study of the diffusion of membrane components in model lipid membranes (GUVs, supported membranes) as well as in biological membranes has become of great interest [59; 100]. Furthermore, many theoretical studies try to describe diffusion behaviour in membranes, in order to develop physical principles ruling it $118 ; 34 ; 94 ; 96$; 97]. They suggest that obstacles (proteins) or heterogenities (domains) influence diffusion processes in membranes. There are several various experimental techniques to study diffusion, Fluorescence Recovery After Photobleaching (FRAP ${ }^{3}$ ) $[1 ;$ 86; 108; 120; 119], Fluorescence Correlation Spectroscopy (FCS) [27; 30; 46; 59; 61; 62; 93; 104], Single-Particle Tracking (SPT) [100; 103], Resonance Energy Transfer (RET) [60], the excimers technique [34], nuclear magnetic resonance (NMR) [10, 65] and electron spin resonance techniques (EPR) [71] and also neutron diffraction [87; 112].

In the research presented here Fluorescence Correlation Spectroscopy (FCS) has been used, where the fluorescence emitted from a very small focus volume $(1 f l)$ is correlated in time. This technique is based on the analysis of the fluctuations in concentration produced by the diffusion of diluted fluorescent species ( $\sim \mathrm{nm}$ concentrations) on the single molecule level. Autocorrelation was pioneered by Wiener as a powerful mathematical tool for noise reduction [127]. Early papers have presented the principle potential of this technique in early 1970's [30; 26; 67]. The development of the confocal illumination scheme by Rigler [93], pushed the sensitivity of this technique to a single molecule (SM) level and led to a renewed interest in FCS. The single molecule measurements are of great interest since they may give information about the physical and chemical properties of a single particle $[12 ; 37 ; 78 ; 100 ; 103$; 113.

FCS has a wide range of applications including the following: the determination of particle concentration [27], translational [30; 46; 59; 104] and rotational [3; 26; 37; 54; 98] mobility in two or three dimensions, chemical kinetics [27; 93; 124], the release of molecules from vesicles in membrane permeability studies [121] as well as the characteristic lifetime of the excited state [25; 126$]$.

\footnotetext{
${ }^{3}$ In FRAP, dyes are photobleached in the central spot by an intense laser light pulse. Because of the random motion, new markers from neighbouring areas diffuse into the bleached region. The rate of arrival in the photobleached spot of fluorescent labelled molecules is measured and the diffusion coefficient is then calculated from the known photobleached spot size and measured diffusion time.
} 
With this method the phase coexistence can be mapped indirectly. With FCS, the fluid and gel domains can be distinguished by the different values of their translational diffusion coefficient $D_{\tau}$. The translational diffusion coefficient in fluid domains $D_{\tau}=3 \cdot 10^{-8} \mathrm{~cm}^{2} / \mathrm{s}$ is faster by about two orders of magnitude in comparison to the diffusion coefficient in gel domains $D_{\tau}=2 \cdot 10^{-10} \mathrm{~cm}^{2} / \mathrm{s}$ [59]. Domain formation is obvious in diffusion experiments as well as in theoretical studies.

The aim of this study is to understand the physical phenomena of diffusion in supported model membranes of phospholipids. This can provide a basis for understanding diffusion processes in more complex biological membranes.

For this purpose a confocal fluorescence setup has been constructed by the author, which is able to record single molecules including fast data acquisition with hardware correlators. A more detailed description of the FCS setup is given in Section 3.1.1. In this thesis, the relation of domain formation to diffusion behaviour in artificial planar membranes using Fluorescence Correlation Spectroscopy and Monte Carlo simulations is presented. The shape of crosscorrelation profiles in the gel and fluid coexistence region suggests that there is not a macroscopic phase separation but gel and fluid microdomains may exist inside large fluid and gel domains. The MC simulations were done in collaboration with Seeger, from the Membrane Thermodynamics and Biophysics Group of MPI for biophysical Chemistry. With Monte Carlo technique the presence of micro- and macrodomains in two-component lipid bilayers could be predicted. The experimental FCS curves are consistent with theoreticallypredicted correlation curves, thus confirming the existence of gel and fluid microdomains inside bigger fluid and gel domains.

The links between the thermodynamic analysis of membrane fluctuations and single molecule experiments provide information on micro-, meso- and nanoscopic domain ${ }^{4}$ formation originating from nearest neighbour interactions in artificial lipid membranes. This study might play an important role in investigating the relationships between the physical properties of membranes and their physiological function.

\footnotetext{
${ }^{4}$ The distinction between the micro- and nanoscopic domains is as follows: microscopic length scale $\leq 1 \mu \mathrm{m}$ : mesoscopic in the range $\approx 10 \mathrm{~nm}-100 \mathrm{~nm}$; nanoscopic length scale $\leq 10 \mathrm{~nm}$.
} 


\section{Chapter 2}

\section{Theory}

\subsection{Diffusion}

Diffusion is a fundamental process by which particles move. It is thus important in biology and medicine, chemistry and geology, engineering and physics and in almost every aspect of our lives. Diffusion is a consequence of the random thermal motion of atoms, molecules, and particles, resulting in a migration of them from areas of high to low concentration. Thus the end result of diffusion is a constant concentration. The speed of diffusing particles depends on the temperature, on the size (mass) of the diffusing particles and on the viscosity of the environment. The energy due to movement is the average kinetic energy, which is itself proportional to temperature.

Einstein has shown that diffusion can be understood as the independent Brownian motion of many particles. The quantitative features of Brownian motion were first described by Robert Brown in 1827. Under the microscope he observed rapid movements of pollen grains suspended in water. This motion is due to the collisions of pollen grains with water molecules, which causing the particles to undergo a random-walk.

Diffusion processes are of great importance in understanding cellular processes. Translational diffusion of lipid molecules in biological membranes became accessible to experimental study some years ago. There are several kinds of possible movements in a lipid bilayer (Figure 2.1).

In lateral diffusion, molecules move laterally within the plane of the membrane. By contrast, in transverse diffusion, which is much less frequent, a molecule moves from one side of the lipid bilayer to the other. In this thesis, the focus will be on lateral diffusion of lipids in a membrane plane. The diffusion process in a lipid membrane is described by the 


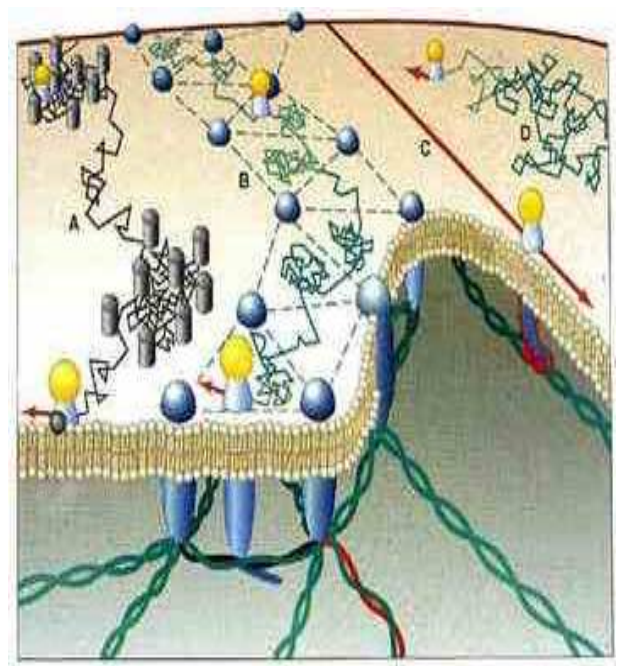

Figure 2.1: Lateral transport modes on the cell surface: A-diffusion restricted by obstacle clusters, B- diffusion restricted by the cytoskeleton (Corral diffusion), C-directed motion and D- free random diffusion [52].

theories presented in the following sub-sections.

\subsubsection{Normal and anomalous diffusion}

The mathematical description of a diffusion process as changes in a concentration is based on Fick's first and second laws, these comprising two differential equations which were known before Einstein. Fick's first law says how quickly particles move from areas of high to areas of low concentration. 1 [1]:

$$
j_{x}=-D_{\tau} \cdot \operatorname{grad} C
$$

where: $j_{x}$ is the current of diffusing particles, equivalent to how many particles are crossing an area of $1 \mathrm{~m}^{2}$ during $1 \mathrm{~s}$,

$D_{\tau}$ is the diffusion coefficient and

$C$ is the concentration.

Fick's second law says how the concentration of particles changes with time at a given point ${ }^{2}$ [103]:

$$
\frac{\partial}{\partial t} C(\vec{r}, t)=D_{\tau} \cdot \nabla^{2} C(\vec{r}, t)
$$

$$
\begin{aligned}
& { }^{1} \operatorname{grad}=\left(\frac{\partial}{\partial x}, \frac{\partial}{\partial y}, \frac{\partial}{\partial z}\right) \\
& { }^{2} \nabla^{2}=\left(\frac{\partial^{2}}{\partial x^{2}}, \frac{\partial^{2}}{\partial y^{2}}, \frac{\partial^{2}}{\partial z^{2}}\right) \text { the Laplacian operator }
\end{aligned}
$$


Assuming that the solvent is isotropic and that diffusion is symmetric in all directions, the probability $P(\vec{r}, t)$ that the molecule under consideration is located at distance $r$ from the origin at time $\mathrm{t}$ for one dimension is equal to:

$$
P(\vec{r}, t)=\frac{1}{\sqrt{4 \pi D_{\tau} t}} \cdot \exp \left(-\frac{r^{2}}{4 D_{\tau} t}\right) .
$$

This latter equation was derived by Einstein. In his Ph.D. work and two subsequent papers [28; 29], Einstein established the theory of Brownian motion. He modelled the Brownian motion according to molecular thermodynamic theory, and connected the macroscopic process of diffusion with the microscopic concept of thermal motion of individual molecules. Extrapolating Eq. 2.3 for $\mathrm{n}$ dimensions gives:

$$
P(\vec{r}, t)=\frac{1}{\left(4 \pi D_{\tau} t\right)^{\frac{n}{2}}} \cdot \exp \left(-\frac{\left|\overrightarrow{r^{2}}\right|}{4 D_{\tau} t}\right) .
$$

Whenever the probability density $P(\vec{r}, t)$ is a Gaussian distribution at long times (large distances), the diffusion coefficient is a well-defined quantity. Determining $D_{\tau}$ depends on used experimental method. It has been common to speak about a macroscopic $D_{\tau}$ and then a normal diffusion, where diffusion is measured over large distances, or about a microscopic $D_{\tau}$ and then anomalous diffusion, where diffusion is measured over short distances. Normal and anomalous diffusion are defined by the dependence of the mean square displacement on time. In homogeneous, two-dimensional systems $(n=2)$ the mean square displacement MSD of a tracer moving randomly is defined by the Einstein-Smoluchowski relation:

$$
\left\langle r(t)^{2}\right\rangle=2 n D_{\tau} t=4 D_{\tau} t
$$

where $\left\langle r(t)^{2}\right\rangle=M S D=\left\langle|r(t+\tau)-r(t)|^{2}\right\rangle$ is the average value of the square of the distance travelled between beginning and end of an experiment.

The average displacement over a time of a measurement $\langle r(t)\rangle=0$, i.e. there is no preferred direction for the random walk. In the case of a normal diffusion, the MSD is proportional to the time $t$. In the case of anomalous diffusion (short distances), the random walk has no more Gaussian distribution and the MSD is no longer linear but is given by [86, 104]:

$$
\left\langle r(t)^{2}\right\rangle=\Gamma \cdot t^{\alpha}
$$


where: $\Gamma$ is the transport coefficient and

$\alpha$ is the temporal exponent $0<\alpha<1$ and $\alpha>1$.

The anomalous diffusion coefficient is defined as:

$$
D_{\text {ano }}=\frac{1}{4} \Gamma \cdot t^{\alpha-1} .
$$

There is also another parameter, the coherence length, which was introduced in two phase systems to distinguish between normal and anomalous diffusion [1]. The coherence length $(\xi)$ is defined as the essential length scale of the typical domains of the minor phase ${ }^{3}$. Diffusion is normal whenever the movement is measured over distances much larger than the correlation length in the system.

\subsubsection{Hydrodynamic theories}

A continuum hydrodynamic model describes diffusion of particles much larger in size than that of the solvent. It is applicable e.g. for lateral diffusion of proteins in a membrane. The hydrodynamic model was applied by Saffman \& Delbrück to analyse Brownian motion of proteins in biological membranes [94]. In this model the membrane is represented as a viscous fluid plane sheet with a viscosity $\mu$ and a very small thickness $h$. It is surrounded by infinite regions of liquids of much smaller viscosity $\mu^{\prime}\left(\mu^{\prime}<<\mu\right)$. The protein molecule is treated as a cylinder with radius $a$, with its axis perpendicular to the plane of the sheet (membrane) (as in Figure 2.2).

Diffusion of a particle due to Brownian motion is described by translation and rotation according to the Einstein equations:

$$
\begin{gathered}
\left\langle r^{2}\right\rangle=4 D_{T} t, \quad D_{T}=k_{B} T b_{T} \quad \text { Translation and } \\
\left\langle\Theta^{2}\right\rangle=2 D_{R} t, \quad D_{R}=k_{B} T b_{R} \quad \text { Rotation, }
\end{gathered}
$$

where: $\left\langle r^{2}\right\rangle$ is the mean square displacement,

$D_{T}, D_{R}$ are the translational and rotational diffusion coefficients respectively,

$k_{B}$ is the Boltzmann constant,

$T$ is the absolute temperature,

\footnotetext{
${ }^{3} \mathrm{~A}$ fluid phase is established as a minor phase which has less mining, a gel phase is established as a major phase.
} 
$b_{T}=\frac{1}{6 \pi \mu a}, \quad b_{R}=\frac{1}{4 \pi \mu a^{2} h}$ are the translational and rotational mobilities respectively [29] and

$\left\langle\Theta^{2}\right\rangle$ is the mean angular rotation in time $t$.

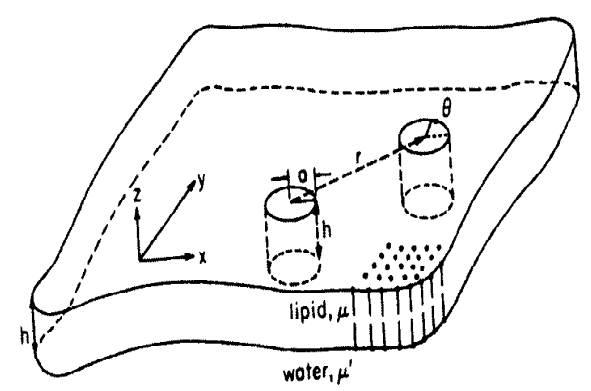

Figure 2.2: The hydrodynamic model. A cylindrical particle embedded in a lipid bilayer membrane bounded by aqueous phases on both sides. The particle is permitted to move laterally in the $\mathrm{x}-\mathrm{y}$ plane and to rotate around the $\mathrm{z}$-axis [94].

The diffusion coefficients are found by assuming finite viscosity of the outer liquid $\left(\mu^{\prime}<<\mu\right)$ and using the frequently-used boundary condition of no slip on the surface of the particle as equal to:

$$
\begin{gathered}
D_{T}=\frac{\left\langle r^{2}\right\rangle}{4 t}=\frac{k_{B} T}{4 \pi \mu h} \cdot\left(\log \frac{\mu h}{\mu^{\prime} a}-\gamma\right) \text { and } \\
D_{R}=\frac{\left\langle\Theta^{2}\right\rangle}{2 t}=\frac{k_{B} T}{4 \pi \mu a^{2} h}
\end{gathered}
$$

where: $\gamma=0.5772$ is Euler's constant.

Hughes has extended Saffman's model for any ratio of the aqueous and membrane viscosities and characterised the system by the dimensionless parameter $\varepsilon=\frac{a}{h} \frac{\mu^{\prime}+\mu_{2}^{\prime}}{\mu} \stackrel{4}{\leftarrow}$. The solution given by Saffman and Delbrück is valid only for $\varepsilon \leq 1$. This is appropriate for model membranes surrounded by water or dilute salt solutions, but may not be valid for biological systems where the bounding solutions are often highly viscous $e . g$. cytoplasm or glycocalix [1]. As mentioned earlier, this model is useful for describing the protein diffusion in model membranes. Vaz et al. $[120 ; 18]$ has tried to apply it to lateral lipid diffusion, but they 
have arrived at the wrong diffusion coefficients. There is another model however, which is applicable for the lateral lipids diffusion in a membrane, this being "free volume theory".

\subsection{3 "Free volume" theories}

A free volume theory was introduced by Cohen \& Turnbull to describe three-dimensional diffusion processes in fluid and glass-formic materials [19]. It has been adopted and changed by Galla for two dimensional translation of lipids in the lipid bilayer [34]. A free volume model takes into account the discreteness of the lipid bilayer. Lipids are placed on a hexagonal lattice where the distance between two lattice points is the average diameter of a lipid molecule. In the free volume model for diffusion in a lipid bilayer, translational diffusion of a lipid molecule occurs when a free volume (area) appears next to the test particle (See Figure 2.3) [66; 119].

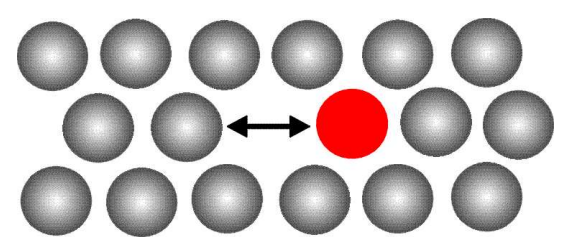

Figure 2.3: Pictorial depiction of displacement in terms of the free-volume model for diffusion in a lipid bilayer. The lipid molecules are shown as spheres in a hexagonal lattice. The unoccupied lattice space represents the free volume and the red lipid molecule the test particle. The double-headed arrow is used to indicate the possible motions of the test particle during the lifetime of the free volume in the lattice space shown [119].

The free volume area should be greater than a certain critical size and the occurrence of free volumes is a result of random density fluctuations in the lipid bilayer. The test particle can move into the vacant space, thus leaving free the space that it occupied originally. The test particle may jump back into its original position, then there will be no "effective displacement". When another lipid molecule moves into the position occupied originally by the test particle this results in "effective displacement" of the test particle. Effective displacements are viewed as discrete jumps in a random walk on a lattice whose characteristic lengths are about the same as the diameter of a lipid molecule. In two-dimensions the average diffusion coefficient is expressed as:

$$
\langle D\rangle=\int_{v^{*}}^{\infty} D(v) p(v) d v
$$

where: $D(v)$ is the diffusion constant inside a free volume $v$,

$p(v)$ is the probability of finding the free volume of size $v$ between $v$ and $v+d v$ and 
$v^{*}$ is the critical free volume.

The probability density $p(v)$ depends exponentially on the free area:

$$
p(v)=\frac{\gamma}{v_{f}} \cdot \exp \left(-\frac{\gamma \cdot v}{v_{f}}\right)
$$

where: $\gamma$ is the geometric factor that corrects for overlap of free-areas, $\frac{1}{2}<\gamma \leq 1$ and

$v_{f}=v_{t}-v^{*}$ is the average free volume in the system, $v_{t}$ being the total average volume per molecule ${ }^{5}$.

The probability of finding the critical free volume $v^{*}$ is:

$$
p(v *)=\exp \left(-\frac{\gamma \cdot v *}{v_{f}}\right) .
$$

Areas below $v^{*}$ are useless for diffusion, i.e. $D(v)=0$ for $v<v^{*}$ and $D(v)=D\left(v^{*}\right)$ for $v>v^{*}$, therefore the expression for the diffusion coefficient becomes:

$$
\langle D\rangle=D\left(v^{*}\right) \cdot p(v *)=D\left(v^{*}\right) \cdot \exp \left(-\frac{\gamma \cdot v *}{v_{f}}\right) .
$$

Consider that the particle loses its sense of direction and, at thermal equilibrium, has an average velocity $u=\sqrt{\frac{2 \cdot k_{B} \cdot T}{m}}$ in a random direction, where $m$ is the mass of the molecule. The time necessary for a jump is equal to $\tau=\frac{\lambda\left(v^{*}\right)}{u}$, where $\lambda\left(v^{*}\right)$ is the molecular diameter (and average "hopping distance"). The jump frequency is equal to $f_{j}=\frac{1}{\tau}=\frac{u}{\lambda\left(v^{*}\right)}$. The diffusion constant is thus given by [34]:

$$
D\left(v^{*}\right)=g \cdot \lambda\left(v^{*}\right) \cdot u=g \cdot \lambda\left(v^{*}\right)^{2} \cdot f_{j},
$$

where $g$ is a geometrical factor $g \cong \frac{1}{4}$ for two-dimensional lattice.

The formation of a free volume is a consequence of the movements of chain segments that are induced thermally. Therefore the jump frequency can be related to the hopping frequency, $f_{h}$, of the defects along the hydrocarbon chains via [34]:

$$
f_{j}=\frac{2 d_{h}^{2}}{L^{2}} f_{h},
$$

where: $d_{h}$ is the lateral displacement of the hydrocarbon chain and

\footnotetext{
${ }^{5} v^{*}$ is a constant, but $v_{t}$ and therefore $v_{f}$ are in general functions of the temperature $v_{f}=\left\langle v_{0}\right\rangle \alpha\left(T-T_{0}\right)$, where $\left\langle v_{0}\right\rangle$ is the mean van der Waals volume equivalent to the free volume, $\alpha$ is the coefficient of thermal expansion and $T_{0}$ is the temperature at which free volume disappears [19].
} 
$L$ is the length of the diffusing molecule.

The hopping frequency is estimated from the activation energy $E_{a}$ of the defects along the hydrocarbon chains that are involved in the formation of the free volume:

$$
f_{h} \approx f \cdot \exp \left(-\frac{E_{a}}{k_{B} T}\right)
$$

where $f$ is the $\mathrm{CH}_{2}$-rocking frequency.

Substitution of Eq. 2.16 into Eq. 2.15 and taking into account the activation energy gives [18]:

$$
\langle D\rangle=A_{0} D^{\prime}\left(v^{*}\right) \cdot \exp \left(-\frac{\gamma \cdot v^{*}}{v_{f}}-\frac{E_{a}}{k_{B} T}\right)
$$

where: $A_{0}$ is a constant dependent only on temperature and

$E_{a}$ is the activation energy per molecule associated with diffusion.

In the case of a lipid bilayer, the activation energy takes into account the interactions of a lipid molecule with its neighbours in the bilayer, the interaction with the bounding fluid and also the energy required to create a hole next to the diffusing molecule, whenever this event is locally associated with an energy change.

\subsubsection{Corral diffusion}

The corral diffusion describes lipid diffusion in a membrane as a random walk in the presence of obstacles e.g. proteins, gel lipid domains around proteins. It has been also called "diffusion in an Archipelago" by Saxton [1; 96; 97]. The presence of an obstacle modifies the diffusion trace of a lipid. The lipid molecule cannot move across proteins and its diffusion coefficient is reduced. Saxton has studied restricted diffusion in a lattice by Monte Carlo simulations. In his study the mobile species (traces) and obstacles occupied sites on the lattice, later being mobile or immobile. Saxton specified the diffusion constant of lipids dependant on the obstacle concentration, size and mobility. When the obstacle size increases, then the diffusion coefficient of a tracer increases. It means that smaller obstacles reduced the diffusion coefficient by less. The mean square displacement, in the case of immobile obstacles, is expressed by the equation: 


$$
\left\langle r^{2}\right\rangle=\left\langle r_{c}^{2}\right\rangle\left[1-A_{1} \cdot \exp \left(-\frac{4 A_{2} D t}{\left\langle r_{c}^{2}\right\rangle}\right)\right]
$$

where: $\left\langle r_{c}\right\rangle$ is the corral size and

$A_{1}, A_{2}$ are geometrical constants of the corral.

In the case of the motion with diffusion, the mean square displacement is given by the equation:

$$
\left\langle r^{2}\right\rangle=4 D t+(v t)^{2}
$$

where $v$ denotes the velocity of the diffusing protein or the velocity of the moving motor in cytoskeleton to which lipids are connected.

As presented here, all of the obstuclers: the proteins and gel-fluid domains, influence the diffusion processes in membranes.

During this work it is attend to understand diffusion processes in a simple artificial lipid mixture containing two lipids with methods from statistical physics. To make the mixture more complicated we would like to introduce additional lipids, e.g., cholesterol, then add proteins. Ultimately we would like to look at diffusion in a living cell.

\subsection{Fluorescence Correlation Spectroscopy}

\subsubsection{FCS Theory}

The fluorescent signal can be separated from the background noise by correlating fluctuations in the intensity of the fluorescent light belonging to the emission from discrete molecules. The method is called Fluorescence Correlation Spectroscopy (FCS) and its concepts have formulated in early 1970s [30; 26; 67]. However autocorrelation was pioneered by Wiener already in 1950s as a powerful mathematical tool for noise reduction [127].

Fluorescence correlation spectroscopy is an elegant and extremely sensitive method which allows single molecule detection, providing information on important molecular properties such as translational $[30 ; 46 ; 59 ; 104]$ and rotational diffusion $[3 ; 26 ; 37 ; 54 ; 98]$, chemical kinetics [27; 93; 124], binding reactions [27; 67], as well as the characteristic lifetime of the excited state [25; 126] (Section 2.2.2).

The usual way to record a fluorescence signal is to illuminate a small volume element (about 
$1 \mathrm{fl}$ ) in order to have only single particles, as shown in Figure $2.4^{6}$ [27]. In work presented here an assumed ellipsoidal 3D-Gaussian observation volume element with a radius of $377 \mathrm{~nm}$ and length of $2020 \mathrm{~nm}$ was illuminated. An average fluorescence intensity consists of events produced by single particles as well as by background noise. Here, the fluctuation of the signals was recorded in discrete time intervals with a temporal resolution.

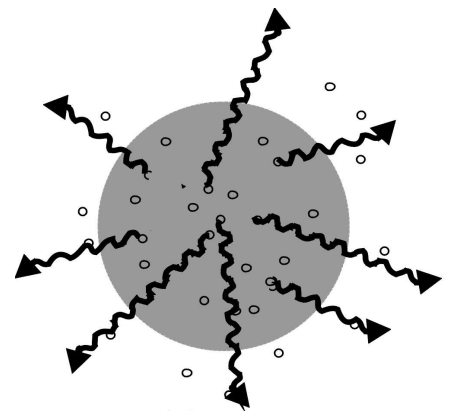

(a)

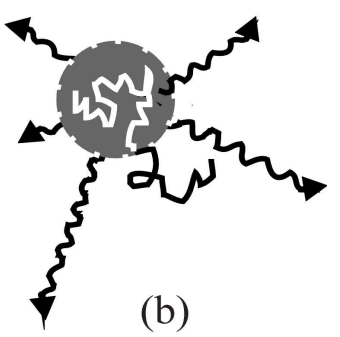

(b)

Figure 2.4: Detecting the fluorescence of excited molecules. In (a), the focal volume $V_{\text {foc }}$ is much larger than the volume of the particle territory $V_{T}$, as is required for obtaining large mean intensities, $V_{f o c}>>V_{T}$. In (b), $V_{f o c}<<V_{T}$, as is required for producing large fluctuations.

When a dye- molecule passes through the focus, it absorbs laser light (changing to the excited state) and after a characteristic time, e.g. $3.9 \mathrm{~ns}$ for Rhodamine R6G, emits a burst of fluorescent light at a different wavelength (see Figure 2.5). The molecule then returns to its original state.

The corresponding signal can be discriminated from the background noise by auto- or cross-correlating the fluorescent light intensity fluctuations $\delta \mathrm{F}(\mathrm{t})$ belonging to the emission from discrete molecules. The intensity recorded at time $t$ is correlated with that recorded at time $(\mathrm{t}+\tau)$. The product is integrated and normalised [27; 104; 123$]$ :

$$
\begin{aligned}
G(\tau) & =\frac{\langle F(t) \cdot F(t+\tau)\rangle}{\langle F(t)\rangle^{2}}=\frac{\langle[\langle F(t)\rangle+\delta F(t)] \cdot[\langle F(t)\rangle+\delta F(t+\tau)]\rangle}{\langle F(t)\rangle^{2}}= \\
& =\frac{\langle F(t)\rangle^{2}+\langle\delta F(t) \cdot \delta F(t+\tau)\rangle}{\langle F(t)\rangle^{2}}=1+\frac{\langle\delta F(t) \cdot \delta F(t+\tau)\rangle}{\langle F(t)\rangle^{2}},
\end{aligned}
$$

where: $\delta F(t)=F(t)-\langle F(t)\rangle$ the fluorescent light intensity fluctuations around the average intensity $\left\langle F\left(t^{\prime}\right)\right\rangle$ and $\langle\delta F(t) \cdot\langle F(t)\rangle\rangle=\langle\langle F(t)\rangle \cdot \delta F(t)\rangle=0$, since $\langle\delta F(t)\rangle=0$.

\footnotetext{
${ }^{6}$ This "particle territory" is the reciprocal of the particle concentration, e.g. for $10^{-9} \mathrm{M}$ solution we have
} $10^{15}$ particles per litre, and thus a particle territory of $10^{-15}$ litre $(1 \mathrm{fl})$. 


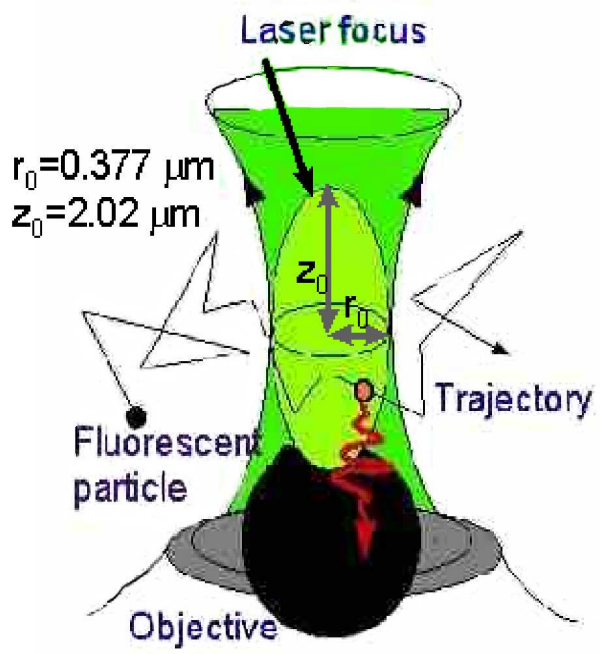

Figure 2.5: Schematic of the laser focus and diffusing particles through it.

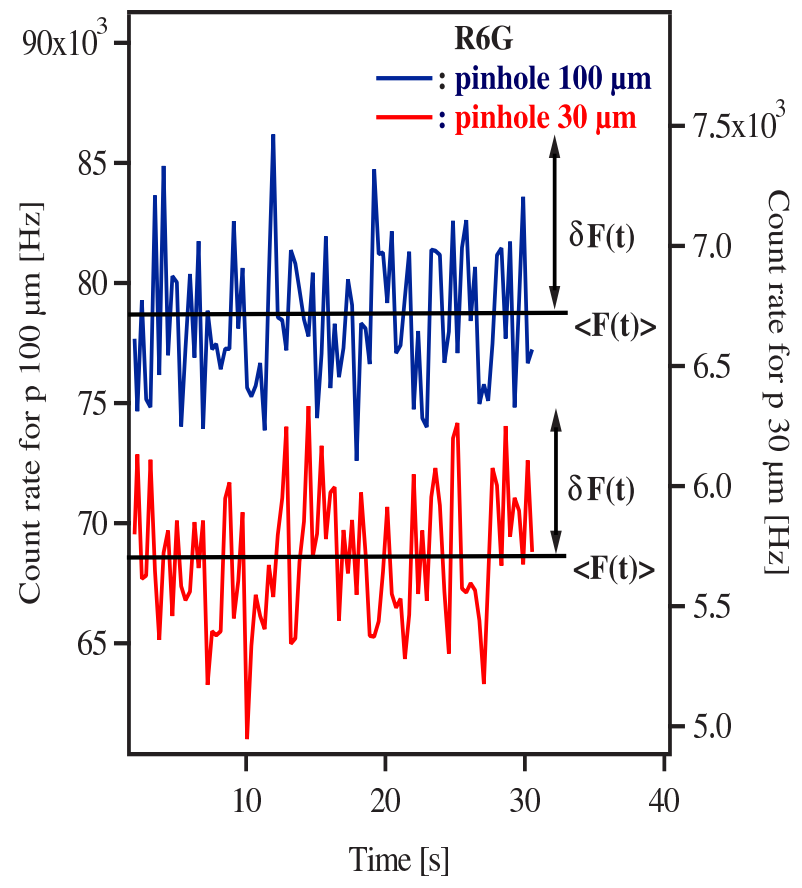

Figure 2.6: Fluorescence intensity fluctuations, $\delta F(t)$, from the average fluorescence, $\langle F(t)\rangle$, of rhodamine $6 \mathrm{G}$ diffusing in bidistilated water at $296.2 \mathrm{~K}$ performed with $100 \mu \mathrm{m}$ (blue colour) and $30 \mu \mathrm{m}$ (red colour) pinhole (p).

Correlation of these both signals shows how much self-similarity they have. If the correlation time $\tau$ is sufficiently small, then the correlation function is close to one, i.e. there is a lot of similarity between a signal recorded at time $t$ and that recorded at time $(t+\tau)$. When 
the correlation time $\tau$ increases, $G(\tau)$ gets values between one and zero, until the correlation time $\tau$ is so long that the both functions have no any common part and than $G(\tau)$ is equal to zero.

From a physical point of view, correlating the fluorescence intensity $F(t)$ is very similar to correlating the fluorescent light intensity fluctuations $\delta F(t)$. The product differs only by 1 , as result of normalisation. The correlation function for the fluorescent light intensity fluctuations $G_{\text {fluct }}(\tau)$ is defined as:

$$
G_{\text {fluct }}(\tau)=G(\tau)-1=\frac{\langle\delta F(t) \cdot \delta F(t+\tau)\rangle}{\langle F(t)\rangle^{2}} .
$$

The fluorescence intensity $F(t)$ is assumed to be proportional to the concentration of fluorescent particles $C(\vec{r}, t)$ that are inside the fixed illuminated volume at that time element $\mathrm{V}$ and to the excitation intensity $I(\vec{r})$, thus:

$$
F(t)=q \cdot Q \int_{V} C E F(\vec{r}) \cdot I(\vec{r}) \cdot C(\vec{r}, t) d r^{3},
$$

where: $q$ signifies the detection quantum efficiency of the detectors and the attenuation of the fluorescence in the passage from the sample to the detector area,

$Q=\sigma_{a b s} \cdot \Phi_{f}$, with: $\sigma_{a b s}$ the excitation cross section of the fluorescent molecules under study and $\Phi_{f}$ their fluorescence quantum yield.

$C E F(\vec{r})$ the collection efficiency function, defined as the fraction of emitted fluorescence at a certain position $\vec{r}$, which passes the pinhole in the image plane [93].

For simplicity, $I_{e m}=C E F(\vec{r}) \cdot I(\vec{r})$ yields:

$$
F(t)=q \cdot Q \int_{V} I_{e m}(\vec{r}) \cdot C(\vec{r}, t) d r^{3} .
$$

Mathematically, the fluorescent light intensity fluctuations $\delta F(t)$ can be expressed as the concentration fluctuations $\delta C(\vec{r}, t)=C(\vec{r}, t)-\langle C\rangle$, where $\langle C\rangle$ is the equilibrium concentration:

$$
\delta F(t)=q \cdot Q \int_{V} I_{e m}(\vec{r}) \cdot \delta C(\vec{r}, t) d r^{3} .
$$

Substituting Eq. 2.25 and Eq. 2.26 into Eq. 2.23 yields:

$$
G_{\text {fluct }}(\tau)=\frac{\langle\delta F(t) \cdot \delta F(t+\tau)\rangle}{\langle F(t)\rangle^{2}}=
$$




$$
=\frac{\int_{V} \int_{V^{\prime}} I_{e m}\left(\vec{r}^{\prime}\right) \cdot I_{e m}\left(\vec{r}^{\prime}\right) \cdot\left\langle\delta C(\vec{r}, t) \cdot \delta C\left(\vec{r}^{\prime}, t+\tau\right)\right\rangle d r^{3} d r^{\prime 3}}{\left(\int_{V} I_{e m}(\vec{r}) \cdot \delta C(\vec{r}, t) d r^{3}\right)^{2}} .
$$

The space-time dependence of the concentration fluctuation $\delta C(\vec{r}, t)$ is given by Fick's second diffusion equation $[28]^{7}$ :

$$
\frac{d}{d t} \delta C(\vec{r}, t)=D_{\tau} \cdot \nabla^{2} \delta C(\vec{r}, t)
$$

here $D_{\tau}$ is the diffusion coefficient of the fluorescent molecules.

The concentration correlation function can be expressed as [123]:

$$
g\left(\vec{r}, \vec{r}^{\prime}, \tau\right)=\left\langle\delta C(\vec{r}, t) \cdot \delta C\left(\vec{r}^{\prime}, t+\tau\right)\right\rangle=\frac{\langle C\rangle}{(4 \pi D \tau)^{\frac{3}{2}}} \cdot \exp \left(-\frac{\left(\vec{r}-\overrightarrow{r^{\prime}}\right)^{2}}{4 D_{\tau} \tau}\right)
$$

The laser focus is assumed to be of Gaussian shape along the axial as well as the radial directions:

$$
I_{e m}=q \cdot Q \cdot I_{0} \cdot \exp \left(-2 \cdot \frac{x^{2}+y^{2}}{r_{0}^{2}}\right) \cdot \exp \left(-\frac{2 z^{2}}{z_{0}^{2}}\right)
$$

where $r_{0}$ and $z_{0}$ denote the distance from the centre of the sample volume element in the radial and axial dimensions respectively, at which the detected fluorescence has dropped by a factor $e^{-2}$ (see Figure 2.5).

The size of the ellipsoidal Gaussian focal volume is given by $V_{f o c}=\frac{4}{3} \pi^{3} \cdot r_{0}^{2} \cdot z_{0}$.

Last years Hess and Webb mentioned that the standard assumption of a three-dimensional Gaussian focal volume is not an accurate approximation [46; 47]. That focal volume has a different shape occurs in the FCS curve in additional exponential component with significant (>30 \%) amplitude and shifts the measured diffusion time as much as $\sim 80 \%$. To minimise these effects and to keep a more nearly Gaussian observation volume, they suggested using

$$
{ }^{7} \nabla^{2}=\left(\frac{\partial^{2}}{\partial x^{2}}, \frac{\partial^{2}}{\partial y^{2}}, \frac{\partial^{2}}{\partial z^{2}}\right) \text { the Laplacian operator }
$$


a small confocal detector aperture and an underfilled objective back-aperture ${ }^{8}$, or two photon excitation or correcting autocorrelation function.

Substituting Eq. 2.29 and Eq. 2.30 into Eq. 2.27, and solving it numerically gives:

$$
G_{\text {fluct }}^{3 D}(\tau)=\frac{1}{V_{f o c} \cdot\langle C\rangle} \cdot\left(\frac{1}{1+\frac{\tau}{\tau_{D}}}\right) \cdot\left(\frac{1}{\sqrt{1+\frac{r_{0}^{2} \tau}{z_{0}^{2} \tau_{D}}}}\right)
$$

with $\tau_{D}$ being the characteristic diffusion time during which a molecule resides in the observation volume of radius $r_{0}$ and length $z_{0}$, given by $\tau_{D}=\frac{r_{0}^{2}}{4 D_{\tau}}$ and the mean number of molecules in the sample volume element is $\langle N\rangle=V_{\text {foc }} \cdot\langle C\rangle$.

In the standard case of an assumption of an ellipsoidal, Gaussian observation volume in the absence of chemical kinetics, the correlation function for the 3-dimensional diffusion (e.g. R6G diffusing freely in water) has the following analytical form:

$$
G_{\text {fluct }}^{3 D}(\tau)=\frac{1}{\langle N\rangle} \cdot\left(\frac{1}{1+\frac{\tau}{\tau_{D}}}\right) \cdot\left(\frac{1}{\sqrt{1+\frac{r_{0}^{2} \tau}{z_{0}^{2} \tau_{D}}}}\right) .
$$

The thickness of the lipid bilayer $(\mathrm{d} \approx 5 \mathrm{~nm})$ is 3 orders of magnitude smaller than the typical axial length of the FCS observation volume $\left(z_{0} \approx 1 \mu \mathrm{m}\right)$, and therefore, the diffusion of a fluorescent label in the membrane can be treated as two-dimensional. For 2-dimensional diffusion, where the focus high $z_{0}>>d$ the correlation function has the following analytical form [104]:

$$
G_{\text {fluct }}^{2 D}(\tau)=\frac{1}{\langle N\rangle} \cdot\left(\frac{1}{1+\frac{\tau}{\tau_{D}}}\right) .
$$

The fluorescence intensity, analysed via the correlation function $G(\tau)$, gives information about:

- the focus size $\left(r_{0}, z_{0}\right)$, which is determined from Rhodamine R6G reference measurements,

- the translational diffusion coefficient $D_{\tau}$ and diffusion times $\tau_{D}$, the half amplitude of the correlation function,

${ }^{8}$ Underfilled objective is characterised by the underfilling factor $\beta>1$, which is the ratio of the back-aperture radius to the beam radius. 
- the mean number of fluorescent molecules $\langle N\rangle$, at concentration $\langle C\rangle$, from the initial correlation amplitude, in a defined observation volume $V_{f o c}$ (see Figure 2.7).

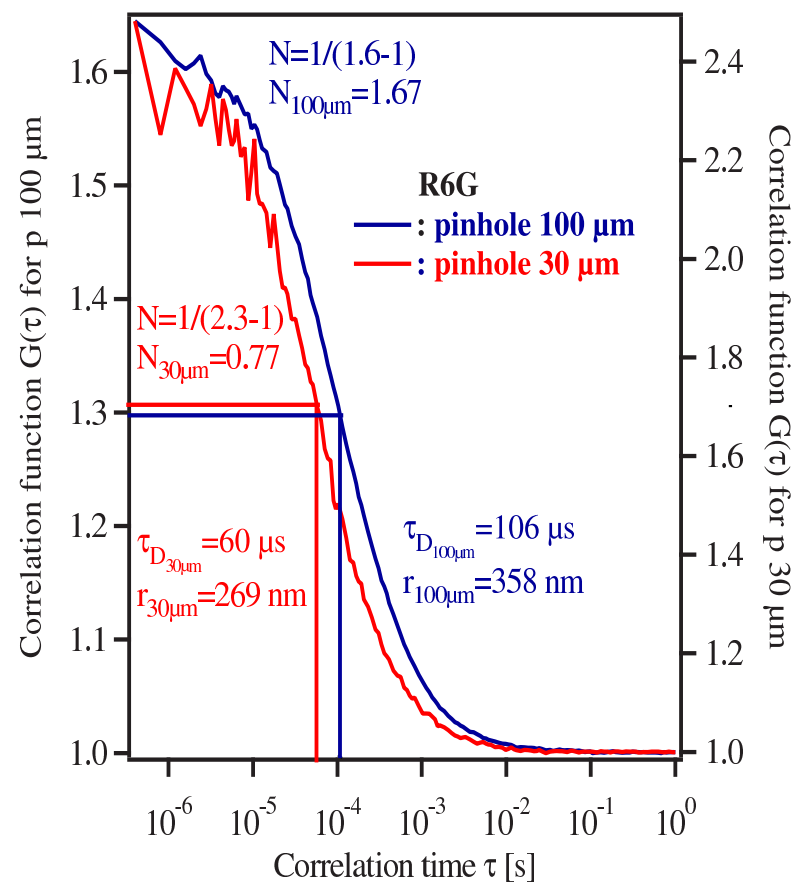

Figure 2.7: Cross-correlation profiles of rhodamine 6G diffusing in bidistilated water saturated with oxygen at $296.2 \mathrm{~K}$ done with $100 \mu \mathrm{m}$ (blue colour) and $30 \mu \mathrm{m}$ (red colour) pinhole (p) corresponding to the fluorescence intensity fluctuations from the Figure 2.6.

It can be seen that the relative fluctuations become smaller with increasing number of molecules measured. The number of molecules in the focal volume can be reduced by diluting the solution or by using a smaller pinhole. However, the fluorescence signal should be still higher than the background signal.

\subsubsection{Labels}

A characteristic property of a dye is the ability to absorb some light in the visible region. Important requirements for dyes are a high absorbing power, a high fluorescence quantum yield as well as low phosphorescence quantum yield and low rates of radiationless decay of triplets. The important radiative transitions appear between two singlet electronic levels, $S_{0}$ and $S_{1}$ (see Figure $2 . \overline{8}$ ) [15; 123$]$. 


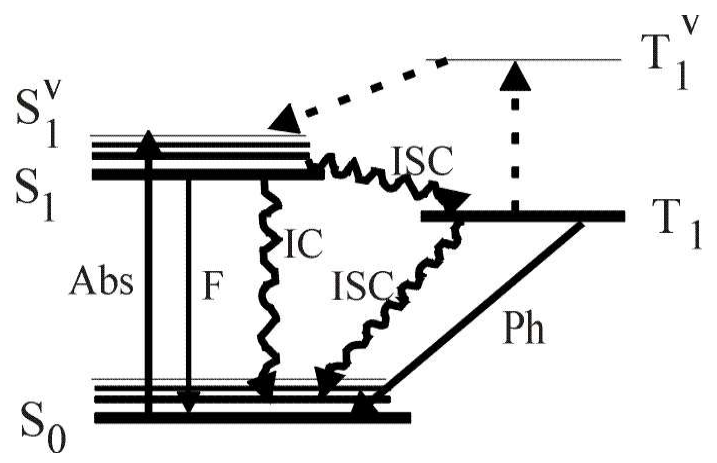

Figure 2.8: Energy level scheme for a dye molecule. Abbreaviations: Abs-absorption, F-Fluorescence, $\mathrm{Ph}-$ Phosphorescence, IC-Internal Conversion, ISC-Intersystem Crossing, S-Singlet state, T-Triplet state [15].

Absorption of a laser photon raises the dye molecule from the ground state $S_{0}$ to its first vibrational excited singlet state $S_{1}^{v}$. First of all the molecule is deactivated very rapidly to the zero-th vibrational level $S_{1}$. Then the excited molecule can release its energy by one of a few processes, the most probably of which is fluorescence, where a dye molecule comes quickly back to the vibrational ground state $S_{0}$ with emission of a photon, $S_{1} \rightarrow S_{0}+h v_{1}$. For rhodamine R6G the fluorescence lifetime is $3.9 \mathrm{~ns}$ [25]. In general $10^{4}$ fluorescence photons can be detected from one label before it bleaches. The other processes cause a less efficient fluorescence emission. The first of them is a radiationless internal conversion (IC), where a label goes to a highly vibrationally-excited ground state $S_{0}^{v}, S_{1} \rightsquigarrow S_{0}^{v}$. The second is a radiationless intersystem crossing (ISC), where energy is transferred to the metastable triplet state $T_{1}^{v}$, lying just below the $S_{1}$ state in energy $\left(S_{1} \rightsquigarrow T_{1}\right)$. One of three processes is possible. The first of them is radiative phosphorescence, where a dye molecule relaxes to the vibrational ground state $S_{0}, T_{1} \rightarrow S_{0}+h v_{2}$ over a longer time scale. For rhodamine $6 \mathrm{G}$ the triplet lifetime is $8 \pm 2 \mu \mathrm{s}[74,100,126]$, whereas for the other labels it can be even milliseconds [15]. The molecule can be also raised to $T_{1}^{v}$ and pass back to the $S_{1}$ state, or can go non-radiative intersystem crossing (ISC) to the highly vibrationally excited singlet state $S_{0}, T_{1} \rightsquigarrow S_{0}$.

For FCS measurements it is important to reduce phosphorescence as much as possible, this being visible on the FCS curves for short time scales. Use of a low laser power prevents a conversion to triplet state, the likelihood of this being a function of excitation power. It is also possible to quench the triplet state by introducing a quencher e.g. oxygen into the system.

For calibration of our system we have used a very common dye, Rhodamine $6 \mathrm{G}$ chloride (R:634), which has a known diffusion constant of $D=3 \cdot 10^{-6} \frac{\mathrm{cm}^{2}}{\mathrm{~s}}$ at $276 \mathrm{~K}$. This aromatic molecule (see Figure 2.9) was purchased from Molecular Probes ${ }^{9}$. The absorption and emis-

\footnotetext{
${ }^{9}$ Eugene/OR, USA
} 
sion spectra of the dye are shown in Figure 2.10. This dye absorbs strongly in the blue-green region, with a maximum at $528 \mathrm{~nm}$, emitting red-shifted light with a maximum at $551 \mathrm{~nm}$ [128].<smiles>CCNc1cc2oc3cc(=[NH+]CC)c(C)cc-3c(-c3ccccc3C(=O)OCC)c2cc1C</smiles>

Figure 2.9: The chemical structure of R6G [128].

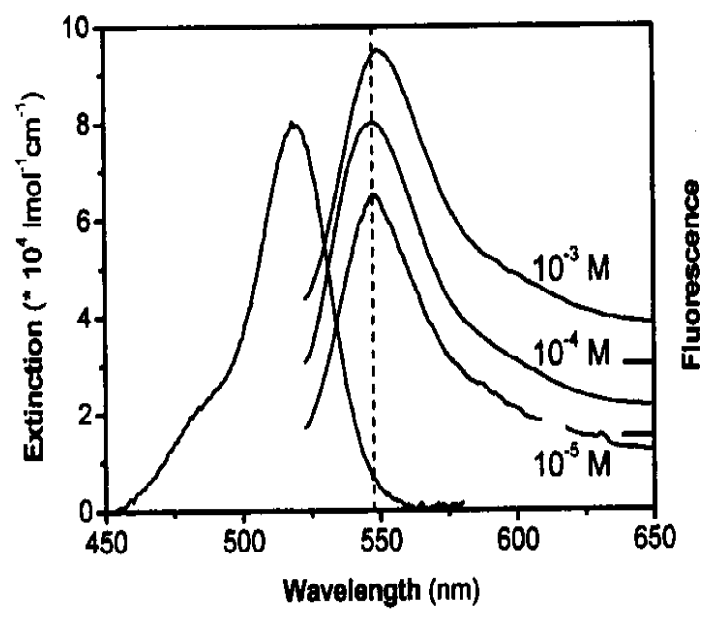

Figure 2.10: Absorption spectrum (on the left) and emission spectra (on the right) at various concentrations of rhodamine $6 \mathrm{G}[128]$.

\subsection{Differential Scanning Calorimetry}

The thermodynamical properties of lipid mixtures such as heat capacities, melting points and melting entropies were examined by Differential Scanning Calorimetry (DSC). The VP$\mathrm{DSC}^{10}$ calorimeter used is a very sensitive instrument, with which it is possible to measure very dilute aqueous suspensions of lipids such as less then $1 \mathrm{mg} / \mathrm{ml}$ of approximately $0.5 \mathrm{ml}$ 
sample volume [90]. A differential scanning calorimeter consists of a sample cell (with a lipid solution) and a reference cell (with a corresponding solvent) as shown in Figure 2.11) placed in an adiabatic shield. In calorimetric measurements we heat the sample and reference cells

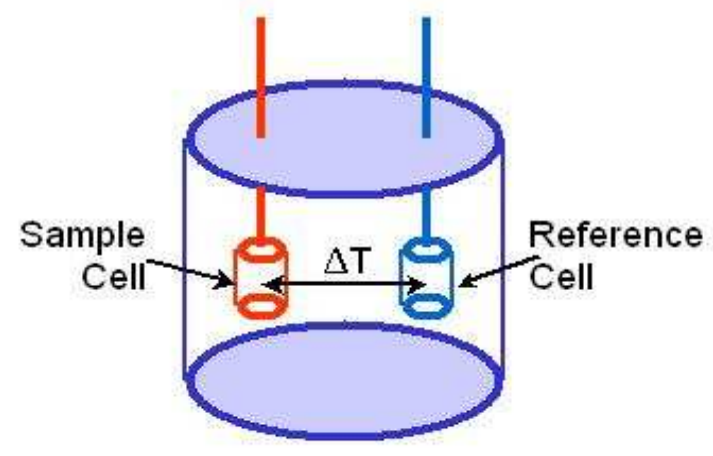

Figure 2.11: Schematic of a differential scanning calorimeter (DSC).

with a constant scan rate $\left(\frac{\Delta T}{\Delta t}\right.$, where $\Delta t$ is the time increment) and keep the temperature difference between them zero. The device records the excess power, $\Delta P=P_{\text {probe }}-P_{\text {reference }}$, at time $t$ and the temporal temperature $\widehat{T}$. Then the excess heat, $\Delta Q$, for small $\Delta t$ is computed as:

$$
\Delta Q=\int_{t}^{t+\Delta t} \Delta P d t \approx \Delta P \cdot \Delta t .
$$

The difference in power which must be supplied to a sample cell and hence the excess heat, $\Delta Q$, at a constant pressure $p$, is proportional to the heat capacity difference $\Delta c_{p}$ :

$$
\left(\frac{d H}{d T}\right)_{p}=\Delta c_{p}=\left(\frac{\partial Q}{\partial T}\right)_{p} \approx \frac{\Delta Q}{\Delta T} \approx \frac{\Delta P}{\frac{\Delta T}{\Delta t}},
$$

where $H$ is the enthalpy.

The data output of the calorimeter is the heat capacity difference as a function of the temperature. Below or above the melting point there is similar rate of heat absorption by both cells, thus resulting in a small difference between lipids and water. During the melting process, the probe absorbs heat at a higher rate than water, resulting in a peak in heat capacity profiles.

\subsubsection{Phase transition}

Differential scanning calorimetry (DSC) makes it possible to determine: 
- $T_{m}$; the phase transition temperature is the temperature at which $50 \%$ of the membrane lipids are transformed from a gel-like structure to a fluid-like structure.

- $\Delta H$; the transition enthalpy is the actual heat required for the entire transition normalised per mole or per unit weight.

- $c_{p}$; the heat capacity is the amount of heat (per mole or per unit weight) which is required to increase the probe's temperature by a given temperature increment (usually, it is defined for $1 \mathrm{~K}$ ).

The chain-melting transition of a one component lipid membrane is a highly co-operative process where the main transition has a half width of $\sim 0.05 K[41]$. The transition temperature itself depends on the lipid species. Membrane lipids with shorter fatty acids or unsaturated fatty acid chains have lower transition temperatures. In the case of shorter lipid chains, the melting transition is broader [101]. $T_{m}$ increases by $15-20 \mathrm{~K}$ for each two carbon atoms extra in the chain length [70]. Moreover, the various polar head groups or the presence of ions can have a dramatic effect [17; 70; 116]. Organisms have membrane compositions consistent with transition temperatures that are somewhat below their lowest body temperature [13; 16, 43]. Lower temperatures tend to decrease membrane fluidity because the molecules of the lipid bilayer tend to form regular, more gel-like structures. As mentioned in Chapter 1 the molecular motion is reduced, i.e. very slow diffusion and little intrachain motion. At higher temperatures, lipids form less regular, more fluid-like structures and the molecules are packed relatively loosely. For this reason there are lots of molecular motions on a faster time scale in comparison with a gel phase. In going from the low temperature (highly ordered acyl chains) to the high temperature (highly disordered acyl chains), the thickness of the bilayer decreases (volume decreases by about $4 \%)^{11}$ as chain melting occurs and the area per molecule increases by about $25 \%{ }^{11}$ (see Figure 2.12) [40; 53; 84.

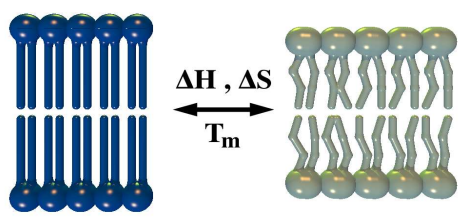

Figure 2.12: The scheme of the phase transition of a gel membrane into a fluid one.

\footnotetext{
${ }^{11}$ Data for DPPC, from [40.
} 


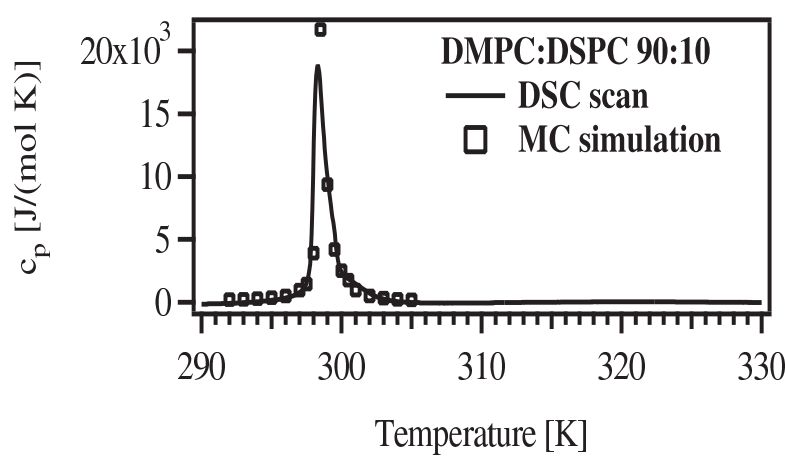

(a)

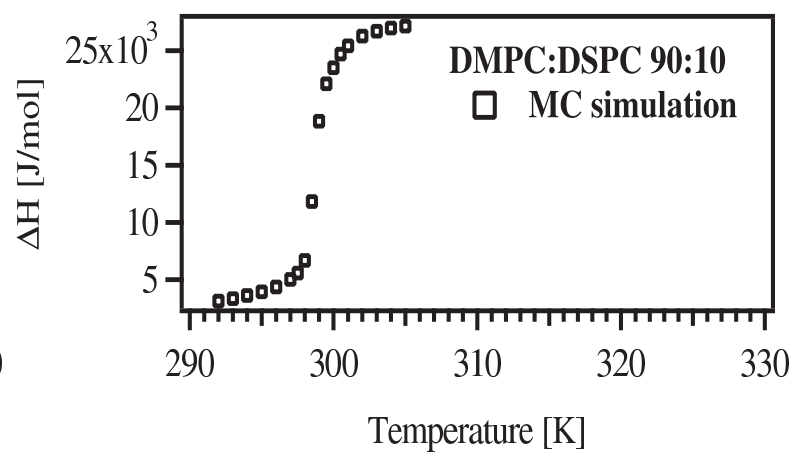

(b)

Figure 2.13: (a): An example of a heat capacity profile for MLVs of DMPC:DSPC 90:10. Scan rate $5 \frac{K}{h}$, $10 \mathrm{mM}$ solution. (b): The corresponding example of enthalpy change calculated according to the fluctuationdissipation theorem.

The main phase transition of lipid vesicles has been considered to be a second-order transition [109]. It means that the extensive thermodynamic variables of the lipid system change continously throughout the whole temperature range (Figure 2.13 (a) and (b)). The extensive variables such as internal energy $U$, enthalpy $H$ and mean number of lipid state chains are variables which depend on the size of the system.

\subsubsection{Theory of the phase diagram}

From a thermodynamical point of view, every substance can exist in one of the following aggregation phases: solid, fluid or gas. Every substance can undergo a phase transformation at given pressures $p$ and temperatures $T$ of the environment. The properties of a mixture of substances in these phases are presented in the form of a phase diagram.

Phase diagrams are applicable also to lipid mixtures since lipids can exist in two phases: a gel (solid) or a fluid one. The phase is represented as a generally homogeneous macroscopic volume inside the system limited by the phase boundaries, however in big systems, the influence of the phase boundaries is neglected. The coexistence of two or more phases in the system is possible. The phase is characterised by a chemical potential $\mu(p, T)$, which has its minimum at the thermodynamical equilibrium. The chemical potential of a one component system is the same at two different phases:

$$
\mu(p, T)=\mu^{\prime}(p, T) .
$$


Eq. 2.36 can be extrapolated for more phases and for more components, e.g. in the case of lipid mixtures, as:

$$
\begin{gathered}
\mu_{1}=\mu_{1}^{\prime}=\cdots=\mu_{1}^{(m-1)} \\
\mu_{2}=\mu_{2}^{\prime}=\cdots=\mu_{2}^{(m-1)} \\
\vdots \\
\mu_{n}=\mu_{n}^{\prime}=\cdots=\mu_{n}^{(m-1)},
\end{gathered}
$$

where $n$ yields the number of components and $m$ yields the number of phases.

There are $n$ different chemical potentials, that make, together with pressures $p$ and temperatures $T, n+2$ variables. The degree of freedom $f$ describing the number of independent variables is given by Gibb's rule as equal to:

$$
f=n-m+2
$$

At constant pressure (as in calorimetric measurements) the constant 2 is reduced to 1 and hence ${ }^{12}$ :

$$
f=n-m+1
$$

For each component a transformation from one phase to the other is possible. The class (order) of transformation is defined as the partial derivative of the chemical potential over temperature. The $n$th order transition yields:

$$
\left(\frac{\partial^{m}}{\partial^{m} T}\right)_{p}\left(\mu^{(i)}-\mu^{(j)}\right) \quad\left\{\begin{array}{l}
=0(m<n) \\
\neq 0(m=n)
\end{array}\right.
$$

where $\mu^{(i)}$ and $\mu^{(j)}$ are the chemical potentials of the phases $i$ and $j$ respectively.

\footnotetext{
${ }^{12}$ For a two component system, at the fluid phase $m=1, n=2$ and $f=2$ so both variables $\mu^{l}$ and $T$ can vary independently and the whole system stays at the fluid phase, at gel and fluid coexistence phase $m=2, n=2$ and $f=1$ so the variable $\mu^{l}$ varies with $T$ and phase separation can be seen. For the gel phase again $m=1$, $n=2$ and $f=2$.
} 
In the case of $1^{\text {st }}$ order transition, the phases are separated so well that there are no phase boundaries from a thermodynamical point of view.

\subsubsection{The ideal solution theory}

Lee tried to describe the phase diagram phenomenologically with an ideal solution theory [63]. Considering an ideal solution of lipids the ruling principles can be defined. In that case two unlike molecules interact in the same way as two similar ones. This means that it does not take into account molecular interaction, even though the phase diagram of an ideal mixture may show phase separation also. Molecules are in the gel state at temperatures lower than the lower limit of the phase coexistence area and in a fluid state at temperatures higher than the upper limit of the phase coexistence area. There is gel and fluid coexistence phase in the lens shaped region in Figure 2.14. The lipid system is at its equilibrium at any temperature,

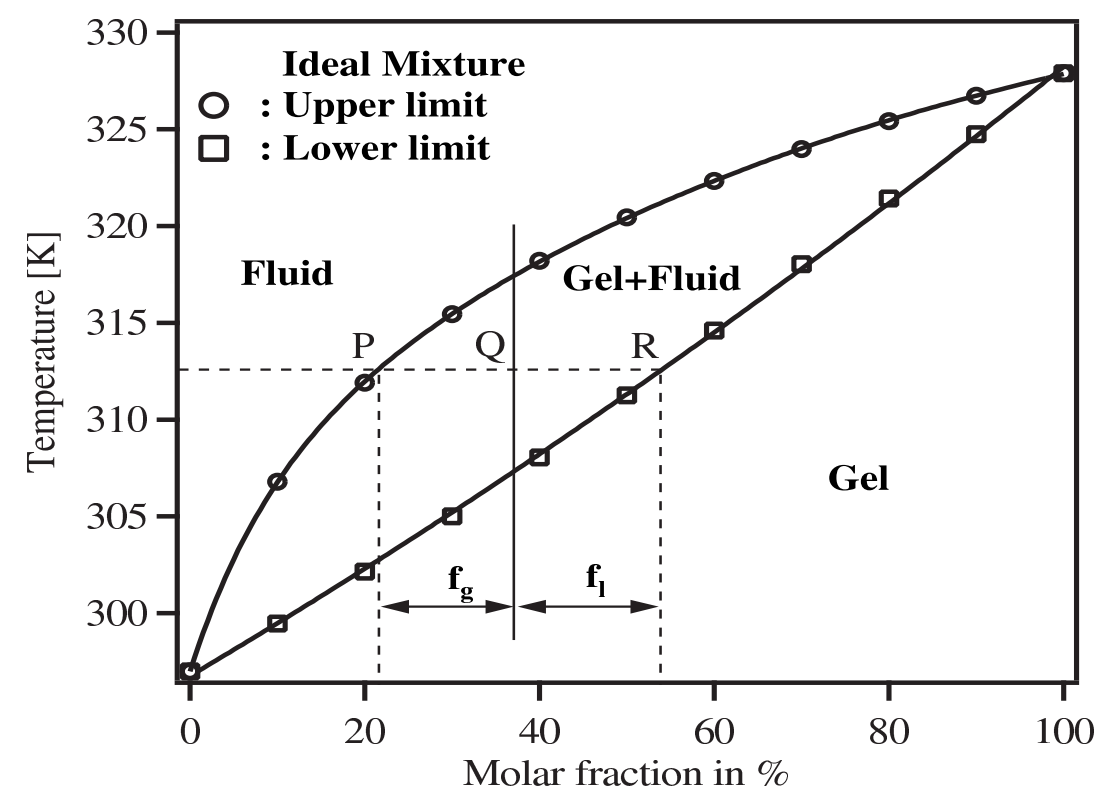

Figure 2.14: The phase diagram of an ideal mixture of the two components (A and B) system.

i.e., the chemical potential is equal for both states of component $\mathrm{A}$ and component $\mathrm{B}$ in phase $l$ and $g$ :

$$
\begin{gathered}
\mu_{A}^{(l)}=\mu_{A}^{(g)} \quad \text { and } \\
\mu_{B}^{(l)}=\mu_{B}^{(g)} .
\end{gathered}
$$

The chemical potential of component $\mathrm{A}$ is given by the equations: 


$$
\begin{gathered}
\mu_{A}^{(g)}=\mu_{A}^{(g)_{0}}+R T \cdot \ln x_{A}^{(g)} \text { and } \\
\mu_{A}^{(l)}=\mu_{A}^{(l)_{0}}+R T \cdot \ln x_{A}^{(l)},
\end{gathered}
$$

where: $\mu_{A}^{(g)_{0}}, \mu_{A}^{(l)_{0}}$ are standard potentials (the standard Gibbs free energy) of one mole of the pure component $\mathrm{A}$ at temperature $T$ under standard conditions (pressure, $p=1 \mathrm{bar}$ ),

$R$ is the ideal gas constant and

$x_{A}^{(g)}, x_{A}^{(l)}$ are the molar fractions of component $\mathrm{A}$ in the gel state and in the fluid state.

The same equations are valid for component B. There is a relationship between molar fractions of the species at equilibrium:

$$
\ln \frac{x_{A}^{(l)}}{x_{A}^{(g)}}=\frac{\mu_{A}^{(g)_{0}}-\mu_{A}^{(l)_{0}}}{R T} .
$$

The partial derivative Eq. 2.45 over temperature shows connections between molar fractions and enthalpy:

$$
\frac{\partial \ln \frac{x_{A}^{(l)}}{x_{A}^{(g)}}}{\partial T}=\frac{H_{A}^{(l)_{0}}-H_{A}^{(g)_{0}}}{R T^{2}} .
$$

This is the van't Hoff equation [4]. The general assumption concerning $c_{p}$ when evaluating the integral of Eq. 2.46 between $T_{A}$ and $T$, is that there is no difference in the heat capacity between both phases. A change $\Delta c_{p}=0$ results in:

$$
\ln \frac{x_{A}^{(l)}}{x_{A}^{(g)}}=-\frac{\Delta H_{A}}{R} \cdot\left(\frac{1}{T}-\frac{1}{T_{A}}\right),
$$

with $\Delta H_{A}=H_{A}^{(l)_{0}}-H_{A}^{(g)_{0}}$.

The transformed Eq. 2.47 yields:

$$
\frac{x_{A}^{(l)}}{x_{A}^{(g)}}=\exp \left[-\frac{\Delta H_{A}}{R} \cdot\left(\frac{1}{T}-\frac{1}{T_{A}}\right)\right] \cong \exp \left(-\mathbf{H}_{\mathbf{A}}\right) .
$$

Similarly for component B:

$$
\frac{x_{B}^{(l)}}{x_{B}^{(g)}}=\exp \left[-\frac{\Delta H_{B}}{R} \cdot\left(\frac{1}{T}-\frac{1}{T_{B}}\right)\right] \cong \exp \left(-\mathbf{H}_{\mathbf{B}}\right) .
$$

The sum of the molar fractions for the same states is equal to one: 


$$
\begin{gathered}
x_{A}^{(g)}+x_{B}^{(g)}=1 \quad \text { and } \\
x_{A}^{(l)}+x_{B}^{(l)}=1 .
\end{gathered}
$$

Transforming Eq. 2.48 and Eq. 2.49 and substituting into Eq. 2.50 yields [63]:

$$
\begin{gathered}
x_{A}^{(l)}=1-x_{B}^{(l)}=\left(1-x_{B}^{(g)}\right) \cdot \exp \left(-\mathbf{H}_{\mathbf{A}}\right) \quad \text { and } \\
x_{B}^{(g)}=\frac{x_{B}^{(l)}}{\exp \left(-\mathbf{H}_{\mathbf{B}}\right)} .
\end{gathered}
$$

Hence the fluid molar fractions are:

$$
\begin{gathered}
x_{A}^{(l)}=\frac{\exp \left(-\mathbf{H}_{\mathbf{A}}\right)\left(\exp \left(-\mathbf{H}_{\mathbf{B}}\right)-1\right)}{\exp \left(-\mathbf{H}_{\mathbf{B}}\right)-\exp \left(-\mathbf{H}_{\mathbf{A}}\right)} \text { and } \\
x_{B}^{(l)}=\frac{\exp \left(-\mathbf{H}_{\mathbf{B}}\right)\left(\exp \left(-\mathbf{H}_{\mathbf{A}}\right)-1\right)}{\exp \left(-\mathbf{H}_{\mathbf{A}}\right)-\exp \left(-\mathbf{H}_{\mathbf{B}}\right)} .
\end{gathered}
$$

The gel molar fractions are:

$$
\begin{gathered}
x_{A}^{(g)}=\frac{\left(\exp \left(-\mathbf{H}_{\mathbf{B}}\right)-1\right)}{\exp \left(-\mathbf{H}_{\mathbf{B}}\right)-\exp \left(-\mathbf{H}_{\mathbf{A}}\right)} \text { and } \\
x_{B}^{(g)}=\frac{\left(\exp \left(-\mathbf{H}_{\mathbf{A}}\right)-1\right)}{\exp \left(-\mathbf{H}_{\mathbf{A}}\right)-\exp \left(-\mathbf{H}_{\mathbf{B}}\right)} .
\end{gathered}
$$

The phase diagram for an ideal mixture is established according to Eq. 2.54, Eq. 2.55, Eq. 2.56 and Eq. 2.57, which are valid only in the phase coexistence region. The values $x_{A}^{(l)}$, $x_{B}^{(l)}, x_{A}^{(g)}$ and $x_{B}^{(g)}$ depend on the temperature. At a given temperature, the ratio of molecules in a fluid phase, $f_{l}$, to molecules in a gel phase, $f_{g}$, is equal to [63]:

$$
\frac{f_{l}}{f_{l}+f_{g}}=\frac{|R-Q|}{|R-P|} .
$$

The points $P, Q$, and $R$ are taken from the phase diagram on Figure 2.14. These points depend on the temperature, however, $Q$ depends also on the ratio of molar fractions of both components. At the temperature $T_{1}$, lying on the upper limit, the ratio of molar fractions of both components is equal to $\frac{x_{A}^{(l)}}{x_{B}^{(l)}}$. By cooling the mixture to temperature $T_{2}$, on the bottom 
limit, the ratio of molar fractions reaches $\frac{x_{A}^{(g)}}{x_{B}^{(g)}}$.

\subsubsection{The regular solution theory}

With an ideal solution only relatively simple phase diagrams can be described. It has been considered that, in general, lipid mixtures should be treated as "regular solutions", instead of an "ideal solutions". The regular solution takes various interaction energies between unlike molecules into account, i.e. $E_{A A} \neq E_{A B} \neq E_{B B}$. The chemical potential of component $\mathrm{A}$ is given by the equation [63]:

$$
\mu_{A}=\mu_{A}^{0}+R T \cdot \ln \left(x_{A} \cdot j_{A}\right),
$$

where $j_{A}$ is the activity coefficient describing how non-ideal the mixture is.

This concept is expressed by an additional term, which is the excess chemical potential $\mu_{A}^{R}$ :

$$
\mu_{A}^{R}=R T \cdot \ln \left(j_{A}\right) .
$$

Hence the excess free energy of the interactions for the mixture is:

$$
G^{R}=x_{A} \cdot \mu_{A}^{R}+x_{B} \cdot \mu_{B}^{R}
$$

There is a trick to find expressions for excess quantities which assumes that the entropy of mixing is like that for the ideal solution:

$$
\Delta S=R\left[x_{A} \cdot \ln \left(x_{A}\right)+\left(1-x_{A}\right) \cdot \ln \left(1-x_{A}\right)\right]
$$

and the enthalpy of the interactions is no longer zero, but is given by:

$$
\Delta H^{R}=\rho_{0}^{(j)} \cdot x_{A} \cdot x_{B}
$$

where $\rho_{0}^{(j)}=z \cdot\left(2 E_{A B}^{(j)}-E_{A A}^{(j)}-E_{B B}^{(j)}\right)$ is the interaction energy parameter with $j \in\{g, l\}$, and $z$ the coordination number of the lipids [63].

Then the following relations, tested by substituting Eq-s. 2.64 and 2.65 into Eq. 2.61, is valid [63]:

$$
\mu_{A}^{R}=\rho_{0}^{(j)} \cdot x_{B}^{2} \quad \text { and }
$$




$$
\mu_{B}^{R}=\rho_{0}^{(j)} \cdot x_{A}^{2}
$$

Substituting Eq-s. 2.48 and 2.49 into Eq-s. 2.60 and 2.59 respectively yields:

$$
\begin{gathered}
\mu_{A}^{(l)}=\mu_{A}^{(l)_{0}}+R T \cdot \ln x_{A}^{(l)}+\rho_{0}^{(l)}\left(1-x_{A}^{(l)}\right)^{2} \text { and } \\
\mu_{A}^{(g)}=\mu_{A}^{(g)_{0}}+R T \cdot \ln x_{A}^{(g)}+\rho_{0}^{(g)}\left(1-x_{A}^{(g)}\right)^{2}
\end{gathered}
$$

Corresponding equations are valid for component B. At thermodynamical equilibrium $\mu_{A}^{(g)}=$ $\mu_{A}^{(l)}$ and $\mu_{B}^{(g)}=\mu_{B}^{(l)}$ are valid:

$$
\begin{array}{r}
\ln \frac{x_{A}^{(l)}}{x_{A}^{(g)}}+\frac{\rho_{0}^{(l)}\left(1-x_{A}^{(l)}\right)^{2}-\rho_{0}^{(g)}\left(1-x_{A}^{(g)}\right)^{2}}{R T}=\frac{-\Delta H_{A}}{R} \cdot\left(\frac{1}{T}-\frac{1}{T_{A}}\right) \quad \text { and } \\
\ln \frac{1-x_{A}^{(l)}}{1-x_{A}^{(g)}}+\frac{\rho_{0}^{(l)}\left(x_{A}^{(l)}\right)^{2}-\rho_{0}^{(g)}\left(x_{A}^{(g)}\right)^{2}}{R T}=\frac{-\Delta H_{B}}{R} \cdot\left(\frac{1}{T}-\frac{1}{T_{B}}\right)
\end{array}
$$

Equations 2.68 and 2.69 can be solved numerically and give values for $x_{A}^{(l)}, x_{A}^{(g)}, x_{B}^{(l)}$ and $x_{B}^{(g)}$ as a function of $x_{A}$ and temperature $T$.

The regular solution theory has disadvantages also, because the free energy $G$ is not defined corrected. It does not take interaction energies between different species of a different state $\left(E_{A B}^{g l}, E_{A B}^{l g}\right)$ into account. Instead, it assumes that species within phases are mixed homogeneously, meaning that no any interfaces between phases exist. These phenomenological theories predict macroscopic phase separation, but not domains formation. Since it is obvious that the structure of membranes is not homogenous, but includes gel and fluid domains [56] it was necessary to find physical principles ruling it. The lipids interactions tried to be described over the complete range of interaction energies, thus including cooperativity parameters between different lipids in a different phases $\left(\omega_{12}^{g l}, \omega_{12}^{l g}\right)$, that corresponds to $E_{A B}^{g l}, E_{A B}^{l g}$. For these purpose Monte Carlo simulations which make use of the thermodynamical properties of lipid mixtures have been done. 


\subsection{Monte Carlo Simulations}

There are several models available for the study of lipid melting and lateral organisation of membranes. On one hand mean-field theories take average energies and entropies of lipid ensembles into account [31]. In this theory only the properties of one, "central" molecule, are treated in details and they are extrapolated on neighbouring molecules. On the other hand, there are numerical methods, i.e. Monte Carlo (MC) simulations. MC simulations give information about fluctuation in membranes, calculate membrane properties like melting enthalpy and entropy and relate them to experimental data. MC simulations have the advantage that they can describe not only the phase diagram, but also provide information on, for example, the magnitude of volume and enthalpy fluctuations. MC simulations can provide a solution to a complex system or problem. Here so-called "coarse-grained" model have been used. This model takes cooperativity parameters between lipids into account and replaced lipids as points, either in a gel or a fluid state, connected with line. The time scales of the simulated processes play no role. The time of the simulated processes can be inferred by comparison of MC simulations with experimental data. MC can be done on a basis of lattice models, either on Ising's two-state, or on Pink's multi-state model, these models being used generally for describing lipid systems. In Pink's model, a lipid can exist in one of ten conformational states. The first is the fully-ordered, all-trans, the last is the fluid conformational state and the other eight are intermediate states $[14 ; 53 ; 81 ; 88$; 89].

\subsubsection{Lattice geometry, states and configuration}

In our case MC simulations were performed using an Ising model ${ }^{13}$ as a basis, proposed by Doniach [23]. This approach has been used also by Sugar et al. [109; 111; 110]. In Sugar's model lipids form a triangular lattice with $N$ lattice points (see Figure 2.15 (a)). Each lattice point is occupied by one chain of either DMPC ${ }^{14}$ or DSPC ${ }^{15}$ respectively. Every lattice point has an individual state, i.e. can exist in either a gel (ordered) or a fluid (disordered) state (see Figure 2.15 (b)). At low temperatures the lipid chains are in the gel ordered phase, whereas at high temperatures the lipid chain order is lost and lipids are in the liquid disordered phase. In this model, each hydrocarbon chain is surrounded by six nearest neighbours (the co-ordination number is $\mathrm{z}=6$ ). The lipid interactions between all species are modelled

\footnotetext{
${ }^{13}$ Ising proposed a magnetic analogy for the representation of the state of the phospholipid molecule: the gel state is a "spin down" state, the fluid state is a "spin-up" state [50].

14 14:0-14:0 1,2-dimyristoyl-sn-Glycero-3-phosphocholine.

15 18:0-18:0 1,2-dimyristoyl-sn-Glycero-3-phosphocholine.
} 
through the interaction energy of the nearest neighbours. The interaction energies include interaction parameters which are given in Eq. 2.79.

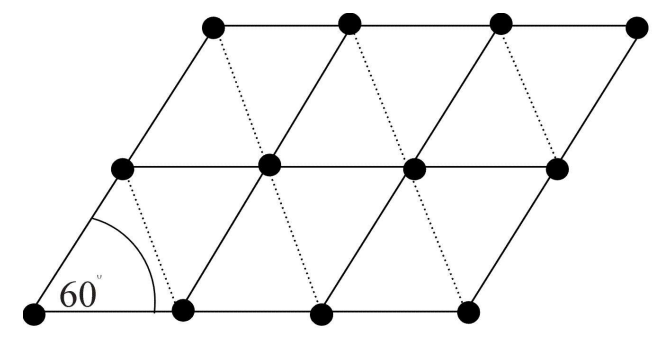

(a)

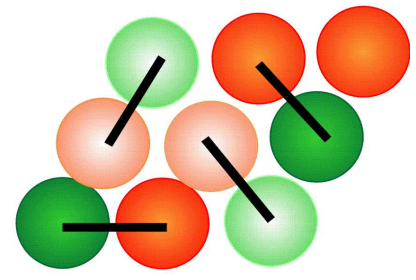

(b)

Figure 2.15: (a) Schema of a triangular matrix. Every lattice point corresponds to a one lipid chain (black symbols). (b) The red symbols represent a gel phase and the green symbols a fluid phase. The dark symbols correspond to DSPC and the light symbols to DMPC. The black lines correspond to the chemical bond.

In the case presented here the triangular lattice is represented by a square matrix $\mathbf{S}$ with $N$ elements, where each matrix element corresponds to a lattice point. There are $N$ lipid chains, where $N_{i}$ is the number of the lipid component $i=1$ (DMPC) or $i=2$ (DSPC) and $N^{m}$ is the number of the lipid component in the state $m=g$ (gel) or $m=l$ (liquid). $N_{i}^{m}$ says how many lipid chains of the $i$ th component are in the $m$ th state. It gives [105]:

$$
\begin{gathered}
N=N_{1}+N_{2}, \\
N=N^{g}+N^{l}, \\
N_{1}=N_{1}^{g}+N_{1}^{l} \quad \text { and } \\
N_{2}=N_{2}^{g}+N_{2}^{l} .
\end{gathered}
$$

The number of pairs of nearest neighbour lipid chains, where one of the chains is of component $i$ in state $m$ and the second chain is of component $j$ in state $n$, is $N_{i j}^{m n}$. The total number of possible pairs of nearest neighbour lipid chains for a two-component system is then $N_{11}^{g g}$, $N_{11}^{g f}, N_{11}^{f f}, N_{22}^{g g}, N_{22}^{g f}$, etc. In the case of periodic boundary conditions, which eliminate the effects of the lattice edge, the following relationships are valid: 


$$
\begin{gathered}
\frac{z}{2} N=N_{11}^{g g}+N_{11}^{g l}+N_{11}^{l l}+N_{12}^{g g}+N_{12}^{g l}+N_{12}^{l g}+N_{12}^{l l}+N_{22}^{g g}+N_{22}^{g l}+N_{22}^{l l} \quad \text { and } \\
z N_{i}^{m}=2 \cdot N_{i i}^{m m}+N_{i i}^{m n}+N_{i j}^{m m}+N_{i j}^{m n}
\end{gathered}
$$

where $i, j \in\{1,2\},(i \neq j)$ and $m, n \in\{g, l\},(m \neq n)$.

It is assumed that lipids interact via van der Waal's interactions which are short-range. That is why only nearest-neighbour interactions between the lattice points are taken into account. Sugar et al. has also tried to incorporate long-range dipole-dipole interactions [111]. Doing this he found out that these interactions do not contribute much to the heat capacity profiles. The matrix configuration $\mathbf{S}$ gives information on the location and the state of lipid species. $E_{i}^{m}$ yields the energies of a lipid chain of a component $i$ in the state $m$ and the energy degeneracy level of component $i$ in the same state is given by $f_{i}^{m}$ in the following definitions. The energies $E_{i}^{m}$ are independent of location and orientation of the rotational isomers in the lipid chains. The $E_{i j}^{m n}$ are the near-neighbour interaction energies between the lipid chain $i$ in state $m$ and the lipid chain $j$ in state $n$, with a degeneracy $f_{i j}^{m n}$. The total free energy of one layer of the bilayer in the matrix configuration $\mathbf{S}$ yields [105; 111]:

$$
G(\mathbf{S})=\sum_{i=1}^{2} \sum_{m=g}^{l} E_{i}^{m} N_{i}^{m}+\sum_{m=g}^{l} \sum_{n=g}^{l} \sum_{i=1}^{2} \sum_{j=1}^{2} E_{i j}^{m n} N_{i j}^{m n} .
$$

Because of various possibilities for the locations and orientations of the rotational isomers in a lipid chain, the total degeneracy is considered as:

$$
f(\mathbf{S})=\prod_{i=1}^{2} \prod_{m=g}^{l}\left(f_{i}^{m}\right)^{N_{i}^{m}} \cdot \prod_{m=g}^{l} \prod_{n=g}^{l} \prod_{i=1}^{2} \prod_{j=1}^{2}\left(f_{i j}^{m n}\right)^{N_{i j}^{m n}} .
$$

For a one component system the excess free energy is given by:

$$
\Delta G(\mathbf{S})=N^{l}(\Delta H-T \Delta S)+N_{11}^{g l} \omega_{11}^{g l}
$$

and for a two component system the excess free energy is given by:

$$
\Delta G(\mathbf{S})=N_{1}^{l}\left(\Delta H_{1}-T \Delta S_{1}\right)+N_{2}^{l}\left(\Delta H_{2}-T \Delta S_{2}\right)
$$




$$
+N_{11}^{g l} \omega_{11}^{g l}+N_{12}^{g g} \omega_{12}^{g g}+N_{12}^{g l} \omega_{12}^{g l}+N_{12}^{l l} \omega_{12}^{l l}+N_{12}^{l g} \omega_{12}^{l g}+N_{22}^{g l} \omega_{22}^{g l},
$$

where:

$$
\begin{gathered}
\Delta H_{i}=\left\{E_{i}^{l}+\frac{z}{2} E_{i i}^{l l}\right\}-\left\{E_{i}^{g}+\frac{z}{2} E_{i i}^{g g}\right\} \quad \text { Enthalpy change, } \\
\Delta S_{i}=k_{B} T \cdot \ln f_{i}^{l}-k_{B} T \cdot \ln f_{i}^{g}-\frac{z}{2} k_{B} T \cdot \ln \left(\frac{f_{i i}^{g g}}{f_{i i}^{l l}}\right) \quad \text { Entropy change, } \\
\omega_{i j}^{m n}=\left[E_{i j}^{m n}-\frac{E_{i i}^{m m}+E_{j j}^{m m}}{2}-k_{B} T \cdot \ln \frac{f_{i j}^{m n}}{\sqrt{f_{i i}^{m m} f_{j j}^{n n}}}\right] \quad \text { Cooperativity parameters. }
\end{gathered}
$$

The probability of finding the configuration $\mathbf{S}$ is given by:

$$
P(\mathbf{S})=\frac{f(\mathbf{S}) \cdot \exp \left(-\frac{\Delta G(\mathbf{S})}{k_{B} T}\right)}{Q\left(N_{1}, N_{2}, T, V\right)},
$$

where: $k_{B}$ is the Boltzmann constant,

$T$ the absolute temperature and

$Q\left(N_{1}, N_{2}, T, V\right)$ is the partition function of the canonical ensemble of the lattice model.

The lipid system is described by ten parameters: two of the enthalpy changes $\Delta H$, two of the entropy changes $\Delta S$ from gel to fluid state and six cooperativity parameters $\omega_{i j}^{m n}$. In the case of an one component system, the magnitude of $\omega_{i i}^{m n}$ defines the cooperativity of the gel-fluid transition. For a sharp transition, the cooperativity is high thus resulting in a high value of $\omega_{i i}^{m n}$. For non-cooperative transition, where each lipid melts independly, the width of the transition is very broad thus resulting in a small value of $\omega_{i i}^{m n}$. In the case of a two component system the influence, of the interaction parameters is much more complicated [4]. 


\subsubsection{Determination of the model parameters}

All model parameters are derived from differential scanning calorimetric experiments on multilamellar vesicles (MLVs, see Figure 2.16). Since diffusion processes on stacks of membranes have been investigated by FCS, parameters were determined from multilamellar vesicles. MLVs consist of several ( 5 or more) bilayers separated by $3 \mathrm{~nm}$ water layer [9]. The following six parameters: the enthalpy changes $\Delta H_{1}, \Delta H_{2}$, melting temperatures $T_{m_{1}}, T_{m_{2}}$ for both lipids, as well as cooperativity parameters $\omega_{11}^{g l}, \omega_{22}^{g l}$ are estimated from two pure systems, either a DMPC or DSPC ${ }^{16}$. The other four cooperativity parameters $\omega_{12}^{m n}$ are delivered from measurements on various mixtures of DMPC:DSPC.

The enthalpy change $\Delta H$ is defined as the mean of the integral of the complete heat capacity profile over temperature.

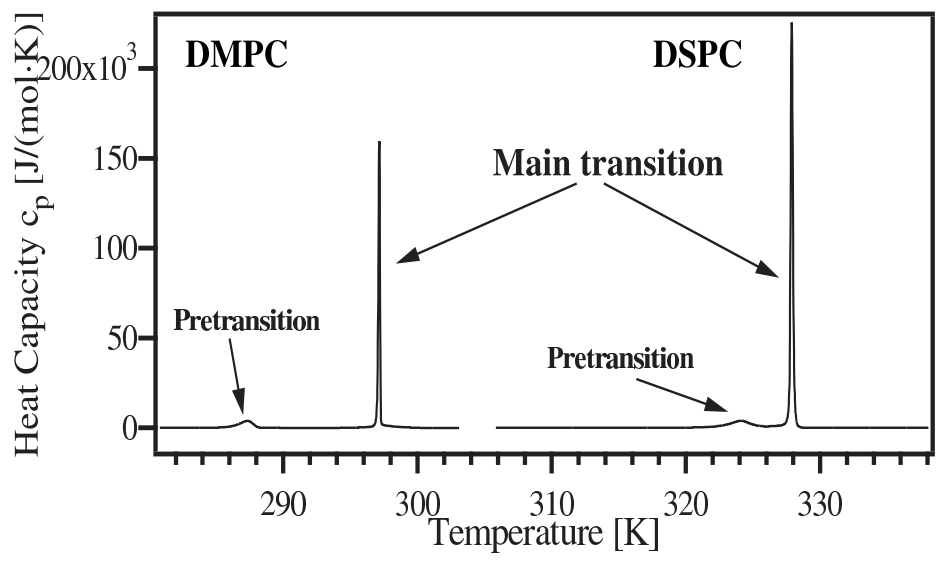

Figure 2.16: Heat capacity curves of MLVs of pure DMPC and DSPC respectively. The pre- and maintransition are shown. The enthalpy change per lipid for DMPC is equal to $\triangle H_{D M P C}=26342 \frac{\mathrm{J}}{\mathrm{mol} \cdot \mathrm{K}}$ and the melting temperature is $T_{m_{D M P C}}=297 \mathrm{~K}$. For DSPC $\Delta H_{D S P C}=50740 \frac{\mathrm{J}}{\mathrm{mol} \cdot \mathrm{K}}$ and $T_{m_{D M P C}}=327.9 \mathrm{~K}$.

The entropy change is determined from the energy change and temperature at the position of the maximum value of the heat capacity curve. At the melting point, the excess free energy (Gibbs free enthalpy) has its minimum [4; 9; 45]:

$$
\Delta G_{i}\left(T_{m_{i}}\right)=\Delta H_{i}-T_{m_{i}} \cdot \Delta S_{i}=0 .
$$

Hence the entropy change is given by:

$$
\Delta S_{i}=\frac{\Delta H_{i}}{T_{m_{i}}}
$$

\footnotetext{
${ }^{16}$ DMPC: 14:0-14:0 1,2-dimyristoyl-sn-lycero-3-phosphocholine, DSPC: 18:0-18:0 1,2-dimyristoyl-sn-lycero-3-phosphocholine,
} 
The cooperativity parameters $\omega_{i j}^{m n}$ are obtained by a comparison of experimental heat capacity curves with simulated ones (see Figure 2.17). The model parameters are listed in Table. 2.1.

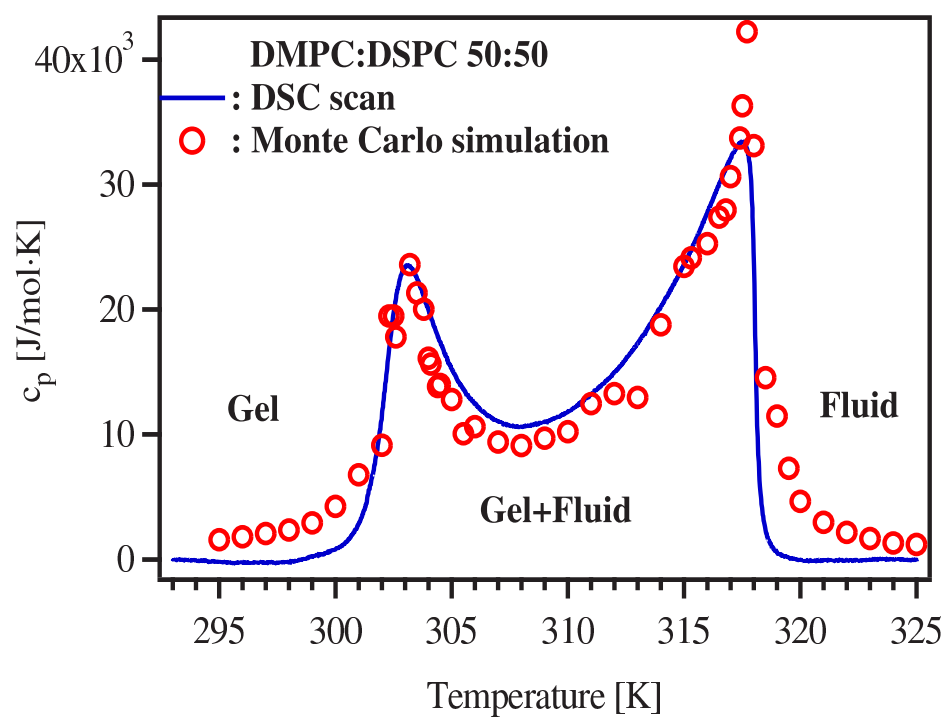

Figure 2.17: Experimental (line) and calculated (points) excess heat capacity curves of DMPC:DSPC 50:50 mixture.

Table 2.1: Model parameters of the two-state two-component DMPC:DSPC bilayer model

\begin{tabular}{|c|c|}
\hline Parameter & $\frac{c a l}{\text { mol-lipidchain }}$ \\
\hline$\Delta H_{1}$ & 3146.5 \\
\hline$\Delta H_{2}$ & 6063.6 \\
\hline$\Delta S_{1}$ & 10.59 \\
\hline$\Delta S_{2}$ & 18.49 \\
\hline$\omega_{11}^{g l}$ & 323.45 \\
\hline$\omega_{22}^{g l}$ & 352.32 \\
\hline$\omega_{12}^{g g}$ & 145 \\
\hline$\omega_{12}^{l l}$ & 60 \\
\hline$\omega_{12}^{g l}$ & 370 \\
\hline$\omega_{12}^{l g}$ & 410 \\
\hline
\end{tabular}

The simulated $c_{p}$-profiles are in quantitative agreement with the measured heat capacity curves over a broad temperature range. The heat capacity curves are reproduced for the mole fraction of the DMPC:DSPC mixture from $10 \%$ to $100 \%$ DSPC adequately (see Figure 2.18 ). 


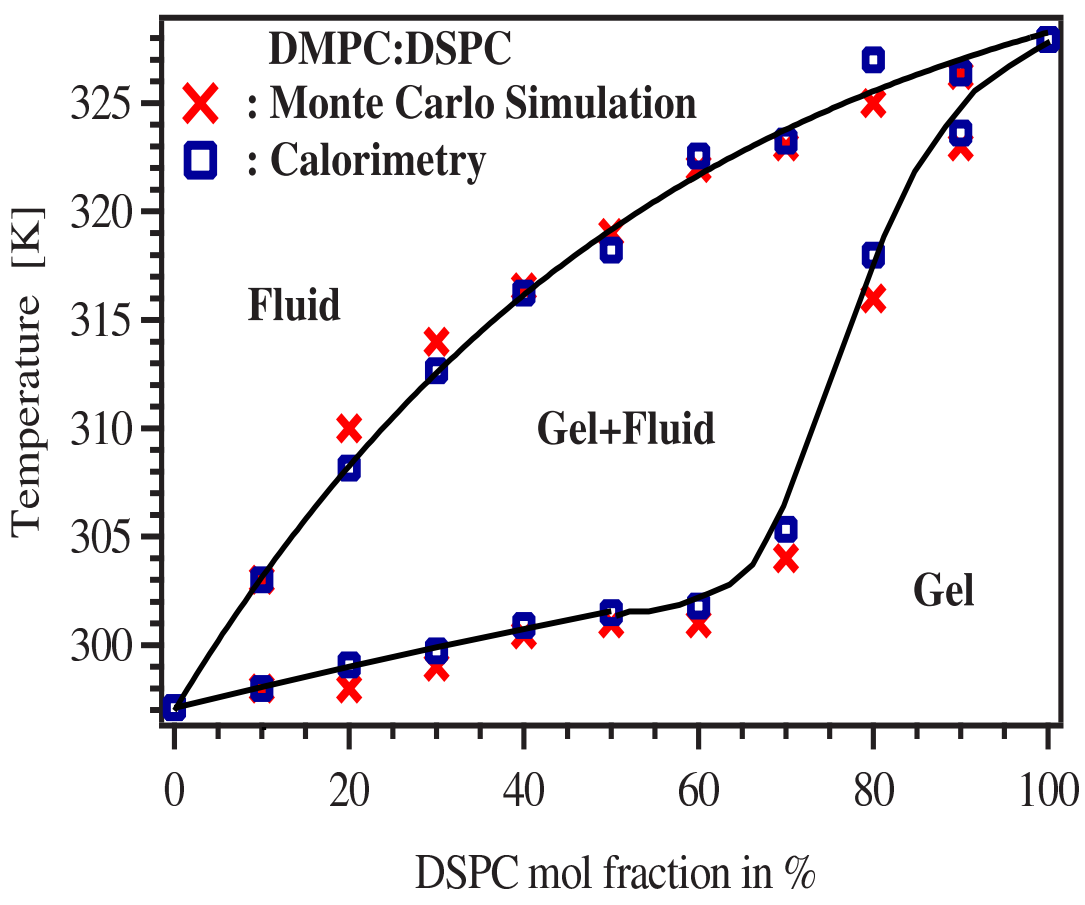

Figure 2.18: DMPC:DSPC phase diagrams. The crosses and squares represent the calculated and experimental data respectively. The points correspond to the melting temperatures at different DSPC mole fractions.

\subsubsection{Steps in the Monte Carlo simulations}

Monte Carlo cycles are performed on the basis of Glauber and Kawasaki algorithms [23]. Initial simulations start from the lattice, where the configurations of the lipid chains' states are evaluated creating a probability $P=\exp -\left(\frac{\Delta H}{k_{B} T}\right)$ which contains information about how many lipids are in the gel ordered phase or in the fluid disordered phase. During one cycle, a chain is picked randomly and its state is changed. The conformation obtained at the end of the first cycle is the initial conformation for the second one. This is repeated consecutively $N$ times, until the system achieves thermodynamic equilibrium. This equilibrium is reached when the enthalpy begins to fluctuate around a mean value. Each cycle is done according to the following order:

- Select a lattice point at random.

- Change its state either from gel to fluid or from fluid to gel.

- Calculate the Gibbs energy difference between the new trial, $\mathbf{S}_{\text {trial }}$, and the old matrix conformation, $\mathrm{S}_{\text {orig, }}$ :

$$
\Delta G=\Delta G\left(\mathbf{S}_{\text {trial }}\right)-\Delta G\left(\mathbf{S}_{\text {orig }}\right)
$$


- Determine the Boltzmannn factor, $K$ :

$$
K=\exp \left(-\frac{\Delta G}{k_{B} T}\right)
$$

- Calculate the probability for the individual lipid to change from the gel state to the fluid state:

$$
P=\left(\frac{K}{1+K}\right)
$$

This is Glauber's algorithm.

- Generate a random number RAN ${ }^{17}$

- Make a decision (YES or NO) on writes to accept the new configuration by comparison RAN with the calculated probability:

$$
\begin{aligned}
& R A N \leq P \rightarrow Y E S \\
& R A N>P \rightarrow N O
\end{aligned}
$$

If the decision is YES, the new trial configuration, $\mathbf{S}_{\text {trial }}$, will be acceptable. If it is NO, the original old configuration, $\mathbf{S}_{\text {orig }}$, will be retained. The decision making is thus the most important step in the whole simulation. The whole process brings the system into thermodynamic equilibrium independently of the choice of the initial conformation (see Figure 2.19). This method of proceedings was first proposed by Metropolis et al [75]. After the equilibrium state is reached, data are accumulated and analysed.

MC cycles simulate thermal fluctuations of the lipid monolayer. The highest fluctuations in the numbers of gel and fluid lipids are in the contacts ${ }^{18}$ between phases and at the melting temperatures. Also, fluctuations in a pure fluid phase are higher than in a pure gel phase. These fluctuations are very useful products of the MC simulation since many important thermodynamics parameters, such as heat capacity and relaxation times, may be derived from them. There are two numerical methods to calculate the $c_{p}$ values from the simulated data. The first uses the partial differential of the mean enthalpy:

\footnotetext{
${ }^{17} \mathrm{RAN}$ is a pseudo random number in the interval from 0 to 1.

${ }^{18}$ There are no borders in the case of phase separation as well as by ideal and regular solution.
} 


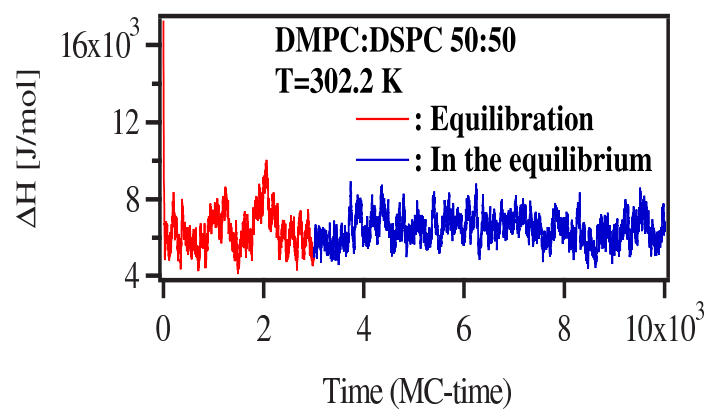

(a)

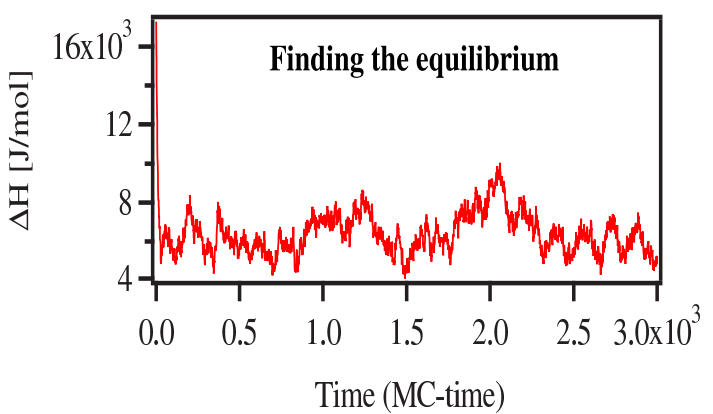

(b)

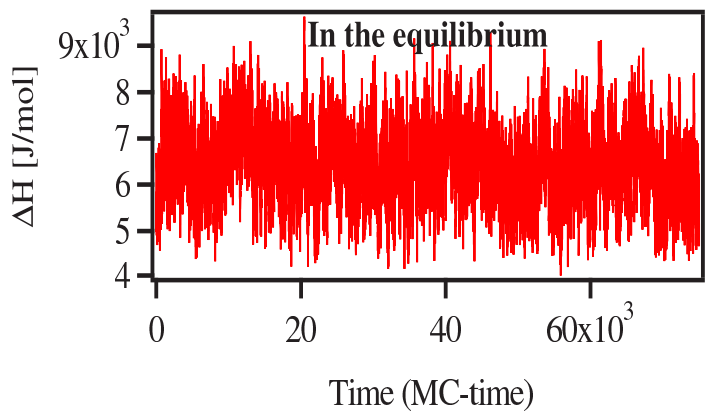

(c)

Figure 2.19: (a) An example of heat capacity performed for DMPC:DSPC 50:50 mixture on 60x60 matrix at $302.2 \mathrm{~K}$. (b) $c_{p}$ fluctuations in going to the thermodynamical equilibrium for the first 3000 cycles and (c) in equilibrium.

$$
c_{p}=\left(\frac{\partial\langle H\rangle}{\partial T}\right)_{p} .
$$

The second uses the fluctuation-dissipation theorem from statistical mechanics to calculate the heat capacity of the system [45]:

$$
c_{p}=\frac{\left\langle H^{2}\right\rangle-\langle H\rangle^{2}}{R T^{2}},
$$

where: $\left\langle H^{2}\right\rangle$ is average of the mean square enthalpy,

$\langle H\rangle$ is the mean enthalpy,

$R=1.987 \frac{\mathrm{cal}}{\mathrm{mol} \cdot \mathrm{deg}}$ the ideal gas constant and

$T$ the absolute temperature. 


\subsubsection{Simulation of FCS experiments}

The aim of this work is to describe the measured Fluorescence Correlation Spectroscopy (FCS) curves of DMPC:DSPC mixtures with a theoretical model. This is intended to leads to a better understanding of diffusion processes, two of which are responsible for the dynamics of the lipid system, swapping and rotation. Swapping is done as an exchange between two molecules selected at random. That means that they do not have to be neighbours, nor do they have to be in the same state or of the same species. This is the Kawasaki algorithm. The achievement of equilibrium in the lateral distribution of molecules is accelerated if no exchange of nearest neighbours occurs [111]. A rotation by $\pm 60^{\circ}$ occurs if the randomlyselected, nearest neighbour chains form a rhombus. Then one of the chains and the chain on the opposite node are exchanged (refer to Figure 2.20). Both processes contribute to the diffusion. Experimental FCS curves are used to estimate the time scales of the simulated diffusion processes. It is known from experiment that lipids in an gel environment diffuse approximately 70 times slower than in a fluid environment. In the FCS experiment a label

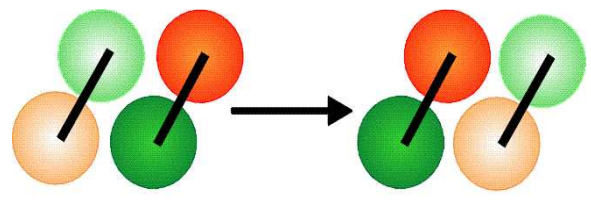

(a)

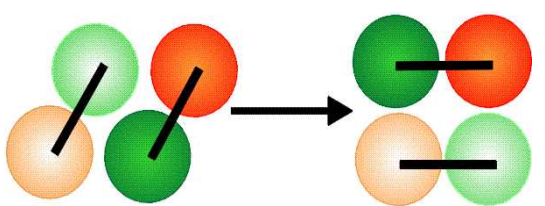

(b)

Figure 2.20: (a) An example of exchange between two lipids i.e. four chains. (b) A schematic of a rotation by $\pm 60^{\circ}$.

diffuses through the laser focus. We can simulate this process by Monte Carlo simulation. After equilibrium (3000 cycles) lipid chains are labelled randomly (see Figure 2.21). The marked chains are assumed to be able of emitting fluorescent light. The state of all neighbours

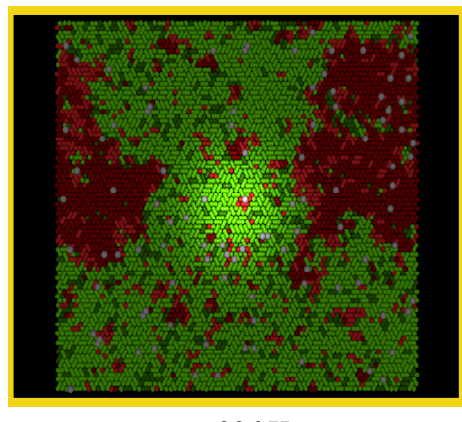

$306 \mathrm{~K}$

Figure 2.21: The lipid snapshot $70 x 70$ of 70:30 DMPC:DSPC mixture at $306 \mathrm{~K}$ with a Gaussian shape laser focus indicated as a light spherical shadow and labels (white spheres). 
of the selected four lipid chains are examined (refer to Figure 2.20). Then the total number of lipid chains $n_{\text {total }}$, plus the number of lipid chains in the gel state $n_{g}$ are counted. The diffusion probability is then calculated according to the equation:

$$
P_{d}=\exp \left(-\frac{n_{g}}{n_{\text {total }}} \cdot \frac{\Delta G_{d}}{k_{B} T}\right)=\exp \left(-\frac{n_{g}}{n_{\text {total }}} \cdot 4.25\right) .
$$

The number $\frac{\Delta G_{d}}{k_{B} T}=4.25$ comes from the distinction between the diffusion time in a gel and in a fluid phase, compared over many FCS correlation profiles ${ }^{19}$.

This probability is chosen in order of getting a difference between the diffusion in a gel and a fluid environment. If $P_{d} \geq R A N$ then one starts calculations with Eq. 2.84 and Eq. 2.80 . If $P_{d} \leq R A N$ then lipids rotate or swap. The first $P_{d}$ needs to be done for the following reasons:

- assuming a pure DMPC gel environment using just Eq. 2.80 would lead to $P=\frac{1}{2}$,

- assuming a pure DMPC fluid environment you would get $P=\frac{1}{2}$ as well. Therefore the same diffusion probabilities would be obtained.

To minimise edge effects, here the square lattice is surrounded by eight replicas. All calculations are done in the central ("original") one. In this simulation, a marked chain can emit one photon immediately after absorption. The predicted fluorescent intensities are analysed after every diffusion step (lipid exchanges and rotations). Intensities are calculated according to a Gaussian profile of the laser focus. Every marked chain gets a certain two dimensional vector $(n, m)$ which says in which matrix the focus sees the label. For example:

$$
\begin{aligned}
& (0,0): \text { original matrix } I=I_{0} \cdot \exp \left[-\frac{\left(x_{P o s}-x_{F o c}\right)^{2}+\left(y_{P o s}-y_{F o c}\right)^{2}}{e x t}\right] \\
& (1,1): \quad I=I_{0} \cdot \exp \left[-\frac{\left(x_{P o s}+L-x_{F o c}\right)^{2}+\left(y_{P o s}+L-y_{F o c}\right)^{2}}{e x t}\right] \text { and } \\
& (n, m): \quad I=I_{0} \cdot \exp \left[-\frac{\left(x_{P o s}+n L-x_{F o c}\right)^{2}+\left(y_{P o s}+m L-y_{F o c}\right)^{2}}{e x t}\right]
\end{aligned}
$$

\footnotetext{
${ }^{19}$ For diffusion in a fluid phase $n_{g}=0 \rightarrow P_{d}=1$; in a gel phase $n_{g}=n_{\text {total }} \rightarrow P_{d}=\exp (-4.25) \cong 0.014$, since diffusion in a gel phase is approximately 70 times slower then in a fluid one.
} 
where: $I_{0}$ is assumed to be equal to 1 ,

$\left(x_{P o s}, y_{P o s}\right)$ are coordinates of the marked chain in the "original" matrix,

$\left(x_{F o c}, y_{F o c}\right)$ are coordinates of the central point of the focus,

ext is the focus extension and

$L$ is matrix size.

Each marked chain has the following intensity:

$$
I_{i}=\exp \left[-\frac{\left(x_{P o s}+n L-x_{F o c}\right)^{2}+\left(y_{P o s}+m L-y_{F o c}\right)^{2}}{e x t}\right] .
$$

Hence the total fluorescent intensity is:

$$
I_{\text {total }}=\sum_{i} I_{i}
$$

The Monte Carlo simulations were performed predominantly on a $60 \times 60$ lattice. Calculations on a larger lattice sizes $(70 \times 70,80 \times 80$ and $200 \times 200)$ were found to give the same results within statistical error of $15 \%$. 


\section{Chapter 3}

\section{Material and Methods}

\subsection{Fluorescence Correlation Spectroscopy}

\subsubsection{FCS Setup}

An important part of this thesis was to construct FCS setup for investigating diffusion processes in membranes, as mentioned in introduction. Therefore the optics principle are described here in a little bit more details. The experimental setup is presented schematically in Figure 3.1 and a brief description is given below. This confocal fluorescence setup consists of a 532-nm green Nd:Yag laser (LASER $2000^{1}$, power $5 \mathrm{~mW}$, beam diameter 0,36 mm) running in $\mathrm{TEM}_{00}$ mode $^{2}$. The excitation light is polarispolarisationed linearly (>200:1 i.e. that less then one photon per 200 will have a perpendicular polarisation). The laser power is regulated by inserting one of five neutral filters (OWIS ${ }^{3}$ ) with the following optical density: 3, 2, 1, 0.6 and 0.2 .

$$
O D=\log _{10}\left(\frac{1}{T}\right)=\log _{10} \frac{\text { Intensity }}{\text { Intensity after OD filter }}
$$

where $\mathrm{T}$ is transmittance.

The calculated transmittances and laser powers with the neutral density filters are shown in Table 3.1. A telescope consisting of two quartz lenses with the focal length $5 \mathrm{~mm}$ and $100 \mathrm{~mm}\left(\operatorname{Linos}^{4}\right)$, magnifies the beam diameter to $7.2 \mathrm{~mm}$. The probe is mounted on an

\footnotetext{
${ }^{1}$ Reno/NV, USA.

${ }^{2} \mathrm{TEM}_{00}$ means a single mode laser, with no mode-hopping.

${ }^{3}$ Staufen, Germany.

${ }^{4}$ Goettingen, Germany.
} 


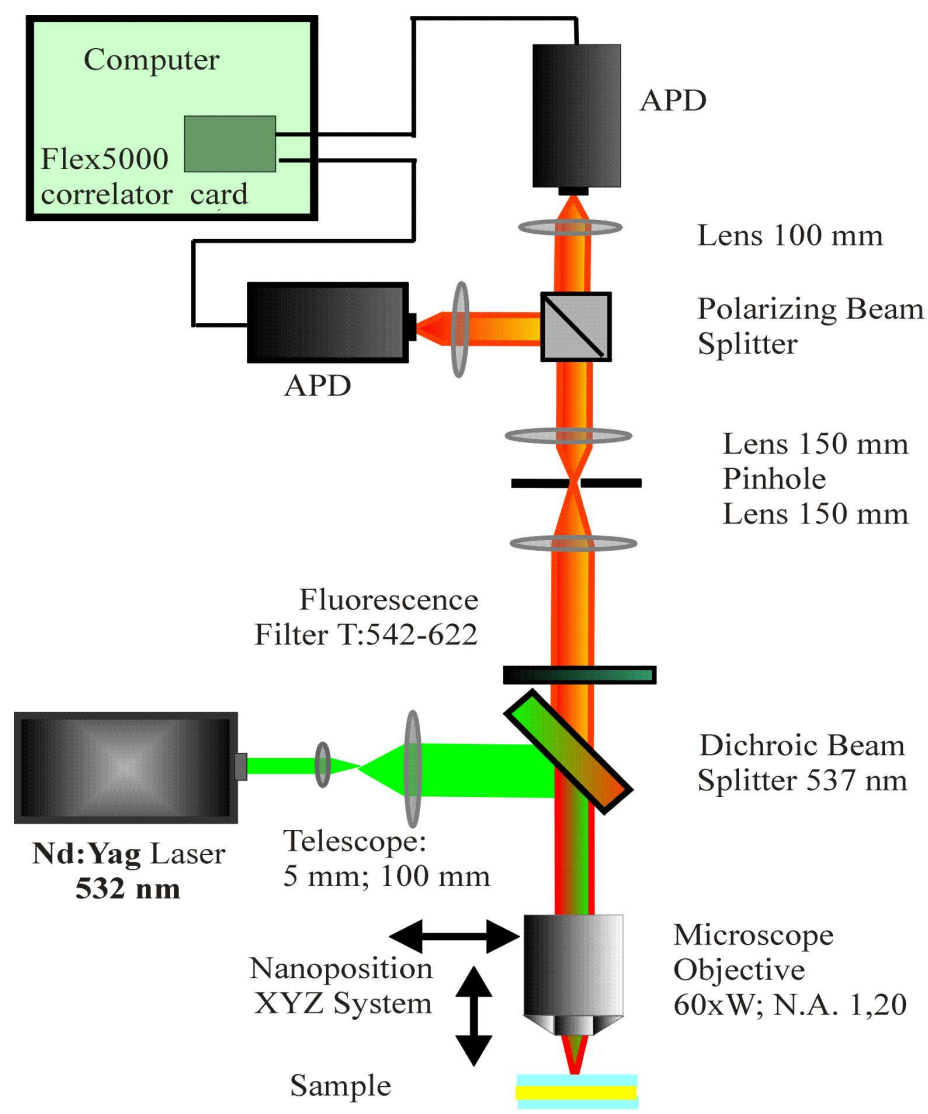

Figure 3.1: The schema of our FCS setup.

Table 3.1: Optical Density Filters

\begin{tabular}{|c|c|c|}
\hline OD Filter & Transmission, - & P-Laser Power, $\mathrm{mW}$ \\
\hline 0 & 1 & 5 \\
\hline 0.2 & 1.58 & 3.16 \\
\hline 0.6 & 3.98 & 1.26 \\
\hline 1 & 10 & 0.5 \\
\hline 2 & 100 & 0.05 \\
\hline 3 & 1000 & 0.005 \\
\hline
\end{tabular}

optical table, equipped with a water immersion objective (Olympus Optical Com ${ }^{5}$, UPLAPO 60xW; N.A.: 1.20; W.D.: $0.25 \mu \mathrm{m}$; F.N.: 26.5; C.C.: 0.13-0.21 $\mu \mathrm{m}$; objective focal length $3 \mathrm{~mm}$ ) and an objective nanoposition XYZ-system ( Fa. Physik Instrumente $\mathrm{GmbH} \& \mathrm{Co}^{6}$, expansion $\leq 100 \mu \mathrm{m})$.

The red fluorescent light is passed through a beamsplitter with a dichroic mirror Q537LP

\footnotetext{
${ }^{5}$ Hamburg, Germany.

${ }^{6}$ Karlsruhe, Germany.
} 
and emission filters (AHF Analysentechnik AG ${ }^{7}$, Transmission: 542-642 nm and 542-622 $\mathrm{nm})$. These band-pass filters discriminate scattered laser light at the excitation wavelength and minimise background from Raman scattered light (Anti-Stokes: 443-455 nm and Stokes: 639-665 nm, [21]) of the water molecules in the sample volume element. The fluorescence is collected through collimating quartz lenses with a $150 \mathrm{~mm}$ focal length (OWIS). In the image plane, a pinhole (OWIS, with diameter $100 \mu \mathrm{m}, 50 \mu \mathrm{m}$ or $30 \mu \mathrm{m}$; large enough to collect virtually all fluorescence excited by the laser illumination in its focal plane) defined the confocal volume element (e.g. for $100 \mu \mathrm{m}$ pinhole, the focus diameter is $0.54 \mu \mathrm{m}$ ).

The polarising beam splitter (Linos; SBB/450-1100 nm) divides the fluorescent light in parallel and perpendicularly polarised light. The light is focused by quartz lenses with a $100 \mathrm{~mm}$ focal length (OWIS). The fluorescence signal is detected by 2 avalanche photo diodes (APD, LASER COMPONENTS GmbH ${ }^{8}$, SPCM-AQR-13) and it is correlated on-line with an FLEX 5000/FAST correlator card (CORRELATOR.com ${ }^{9}$ ). By the use of two detectors we can eliminate all inherent noise due to the detectors (dark current) as long as the detectors themselves are not correlated (e.g. by using cross-correlation mode where the signal from one detector is correlated with the signal from the second one).

\subsubsection{Calculations for telescope lenses and pinhole}

For the construction of an FCS setup, firstly the appreciate optics system (lenses, mirrors, filers, pinholes) should be decided upon. For this it is necessary to calculate the focal lengths of the various lenses needed for the system. Using a standard OLYMPUS objective lens with a focal length of $180 \mathrm{~mm}$, the following calculations were made:

$$
\text { Objective focal length }=\frac{\text { tube lens focal length }}{\text { magnification }}=\frac{180}{60}=3 \mathrm{~mm} \text { and }
$$

Diameter of the back focal plane (diameter of laser beam coming into objective) $=$

$$
=2 \cdot \text { Numerical aperture } \cdot \text { focal length }=2 \cdot 1.2 \cdot 3=7.2 \mathrm{~mm} \text {. }
$$

The objective must have a high transmission efficiency, must not contribute fluorescence from its internal parts, should have a high light collection efficiency and good resolution. These criteria are satisfied with an objective having a high numerical aperture (N.A.). The numerical

\footnotetext{
${ }^{7}$ Tubingen, Germany.

${ }^{8}$ Olching, Germany.

${ }^{9}$ Bridgewater/NJ, USA.
} 
aperture of a lens is defined as the effective collection angle of a lens, a lens collecting light from a sample, through air, can only capture that portion of light limited by the reflection angle (Snell's law) [61]:

$$
N . A .=n \cdot \sin \Theta \text {, }
$$

where: $n$ is the reflective index of intervening media for air $n=1$, for water $n=1.33$, for glycerol $\mathrm{n}=1.47$ and for microscope immersion oil $\mathrm{n}=1.52$ and

$\Theta$ the half collection angle.

For the water immersion objective used in that setup, the half collection angle is equal to $65^{\circ}$, which gives better light collection and a greater field of view compared to the objective with air as the medium. For the isotropic light emission, only one-third of the emitted light will be collected $\left(\frac{1}{3} \cdot 4 \cdot \pi=\frac{1}{3} \cdot 4 \cdot 90^{\circ}=120^{\circ}\right)$ as shown in Figure 3.2. [61].

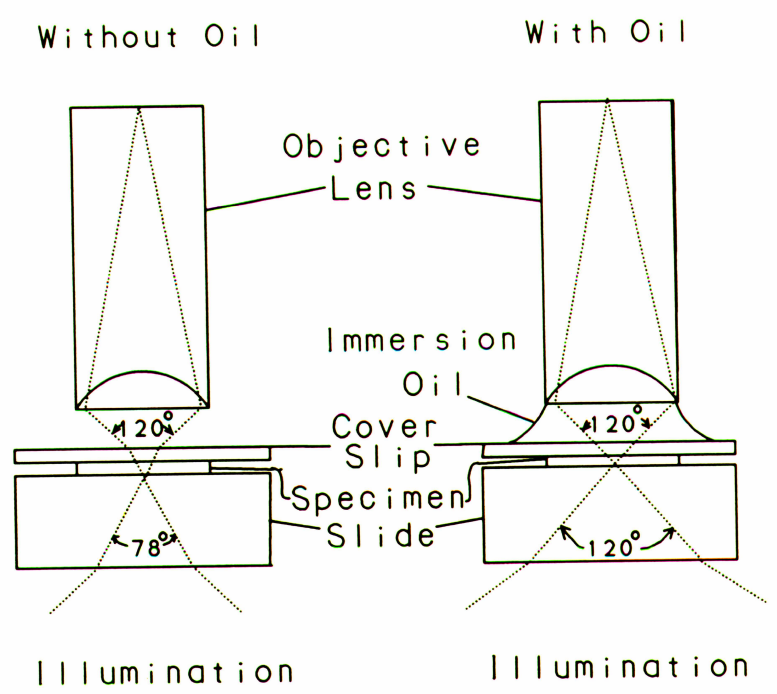

Figure 3.2: The effective acceptance angle at the objective lens with and without immersion oil [61].

The collection efficiency $\psi$ is given by the equation:

$$
\psi=\frac{1}{2} \cdot(1-\cos \Theta)=0.29
$$

It means that the objective collects only $29 \%$ of all photons emitted in whole space $(4 \pi)$. At the laser beam exit, the diameter of the beam is $0.36 \mathrm{~mm}$. The back focal plane of the objective is equal to $7.2 \mathrm{~mm}$. The beam expander was placed between laser and objective to increase the beam diameter. The focal lengths of the beam expansion lenses have the 
following values:

$$
\begin{gathered}
f(\text { lens } 1)=\frac{0.36 \mathrm{~mm}}{7.2 \mathrm{~mm}} \cdot f(\text { lens } 2) \text { and } \\
f(\text { lens } 1)=\frac{1}{20} \cdot f(\text { lens } 2) .
\end{gathered}
$$

Lenses with focal lengths of $5 \mathrm{~mm}$ and $100 \mathrm{~mm}$ respectively were chosen. The focal diameter (Rayleigh distance) of an objective is equal to:

$$
\frac{1.22 \cdot \lambda_{\text {laser }}}{N . A .}=\frac{1.22 \cdot 532 \mathrm{~nm}}{1.2} \cong 0.541 \mu \mathrm{m}
$$

The pinhole diameter corresponding to an air disc was calculated as being equal to:

$$
\text { Focal diameter of an objective } \cdot \text { magnification }=0.541 \mu \mathrm{m} \cdot 60 \cong 33 \mu \mathrm{m} \text {. }
$$

A point inside the focal volume of a half high $z_{0}$ emits a light in all directions. The emitted light was collected through the objective and in the pinhole plane, the point was represented as an circular shaped image with a radius $R$. The sharpest image and the smallest radius, $R(z=0)=R_{0}$, belong to the points placed in the focal plane $\left(z_{0}=0\right)$ (see Figure 3.3) [24]. The pinhole in the image plane blocks any fluorescent light not coming from the focal region thus providing better axial resolution.

Is that image small enough that the whole fluorescence signal is focused on an active area of APD, which is equal to $176 \mu \mathrm{m} \times 176 \mu \mathrm{m}$ ? Lenses focusing light on a pinhole have a focal diameter of $150 \mathrm{~mm}$, and focusing light on an APD have focal lengths of $100 \mathrm{~mm}$ or $20 \mathrm{~mm}$. The image diameter was calculated according to the equation:

$$
\text { pinhole diameter } \cdot \frac{\text { focal length of the APD lens }}{\text { focal length of the pinhole lens }}
$$

for a $100 \mu \mathrm{m}$ pinhole:

$$
\begin{gathered}
100 \mu \mathrm{m} \cdot \frac{100 \mathrm{~mm}}{150 \mathrm{~mm}}=100 \mu \mathrm{m} \cdot 0.667=67 \mu \mathrm{m} \quad \text { and } \\
100 \mu \mathrm{m} \cdot \frac{20 \mathrm{~mm}}{150 \mathrm{~mm}}=100 \mu \mathrm{m} \cdot 0.133=13 \mu \mathrm{m}
\end{gathered}
$$



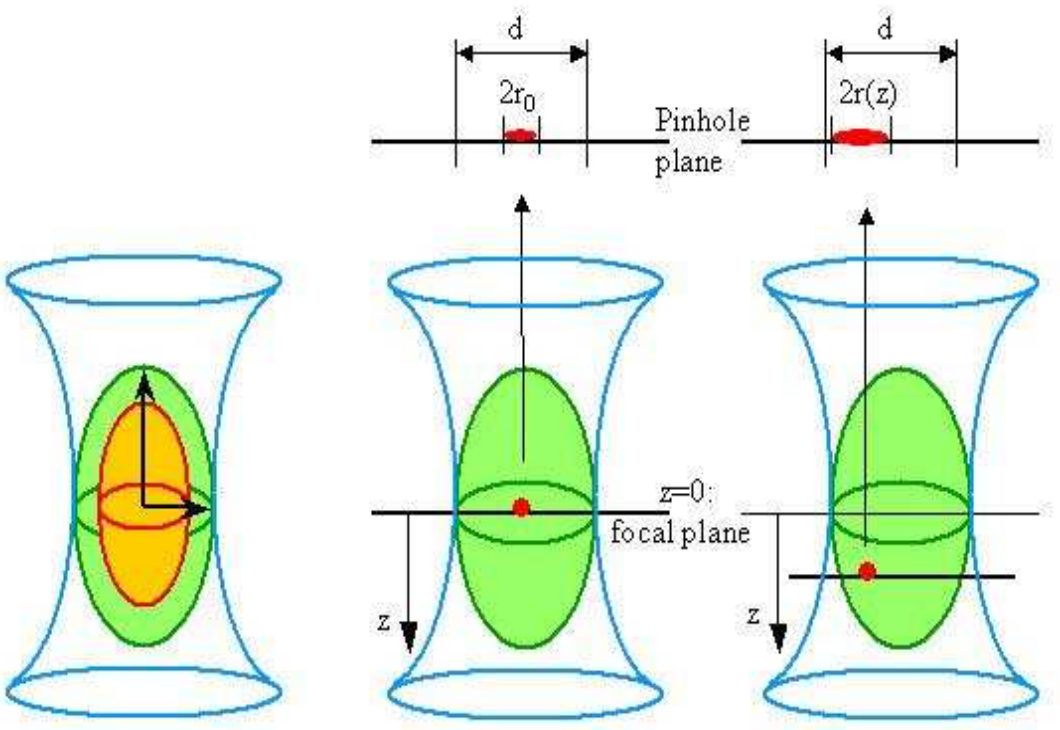

Figure 3.3: The function of the pinhole.

For a $30 \mu \mathrm{m}$ pinhole, the image diameters are equal to $20 \mu \mathrm{m}$ and $4 \mu \mathrm{m}$ for a $100 \mathrm{~mm}$ and $20 \mathrm{~mm}$ lens respectively. So all of the fluorescent light is focused on the active area of the photodiode.

\subsubsection{Focus size}

The dimensions of the laser beam focus, the diameter of the pinhole in the image plane of the confocal setup, the magnification and the numerical aperture of the microscope objective define the sample volume from which fluorescent light is collected. The radius of focus, $r_{0}$, and the mean molecule number, $\langle N\rangle$, are determined from the translational diffusion time of $10 \mathrm{nM}$ rhodamine $6 \mathrm{G}$ solution (with a known diffusion coefficient equal to $D_{\tau}=3 \cdot 10^{6} \mathrm{~cm}^{2} / \mathrm{s}$ at $296 \mathrm{~K}$ [33]), obtained from FCS measurements as follow:

$$
D_{\tau}=\frac{r_{0}^{2}}{4 \cdot \tau_{D}} \Rightarrow r_{0}=\sqrt{4 \cdot D_{\tau} \cdot \tau_{D}}
$$

The radius was taken as the mean value of the radii measured by both avalanche photodiodes (A and B) $\left\langle r_{0}\right\rangle=\frac{r_{A}+r_{B}}{2}$ and its standard deviation is given by $\sigma_{\left\langle r_{0}\right\rangle}=\sqrt{\frac{\sigma_{r_{A}} \cdot \sigma_{r_{A}}+\sigma_{r_{B}} \cdot \sigma_{r_{B}}}{2}}$. The mean values of the radius $\left\langle r_{0}\right\rangle_{0}$ and their standard deviations $\sigma_{\left\langle r_{0}\right\rangle}$ for different optical density filters and both pinholes are presented in Table 3.2 .

Then, the mole number in a focus is estimated. Since 1 Mole has $6.023 \cdot 10^{-23}$ molecules, 
Table 3.2: The mean radius $\left\langle r_{0}\right\rangle_{0}$ and its standard deviations $\sigma_{\left\langle r_{0}\right\rangle}$ for $100 \mu \mathrm{m}$ and $30 \mu \mathrm{m}$ pinholes.

\begin{tabular}{|c|c|c|}
\hline $\begin{array}{l}\text { Pinhole } \\
\text { OD Filters }\end{array}$ & $\begin{array}{c}\left\langle r_{0}\right\rangle \\
\cdot 10^{-7} \mathrm{~m}\end{array}$ & $\begin{array}{c}\sigma_{\left\langle r_{0}\right\rangle} \\
\cdot 10^{-7} \mathrm{~m}\end{array}$ \\
\hline $100 \mu \mathrm{m} ; 2$ & 3.62 & 0.10 \\
\hline $100 \mu \mathrm{m} ; 1$ & 3.77 & 0.16 \\
\hline $100 \mu \mathrm{m} ; 0.6$ & 4.28 & 0.32 \\
\hline $100 \mu \mathrm{m} ; 0.2$ & 5.81 & 0.39 \\
\hline $30 \mu \mathrm{m} ; 2$ & 2.60 & 0.25 \\
\hline $30 \mu \mathrm{m} ; 1$ & 2.86 & 0.21 \\
\hline $30 \mu \mathrm{m} ; 0.6$ & 3.39 & 0.31 \\
\hline
\end{tabular}

than the mean molecule number, $\langle N\rangle$, determined from R6G measurement corresponds to a mole number:

$$
\text { mole number }=\frac{1 \text { mole } \cdot\langle N\rangle}{6.023 \cdot 10^{-23}} \text {. }
$$

From the calculated mole number and from the known rhodamine R6 solution of $10 \mathrm{nM}$ per litre e a focal volume, $V$, is estimated as being equal to:

$$
V=\frac{\text { mole number } \cdot 10^{-3} \mathrm{~m}^{3}}{10 n M} .
$$

It is assumed that the focal volume, for the highest intensity, has an elliptical shape:

$$
V=\frac{4}{3} \pi r_{0}^{2} z_{0} \Rightarrow z_{0}=\frac{3 V}{4 \pi r_{0}^{2}}
$$

The mean half lengths $\left\langle z_{0}\right\rangle$ of the focal volume and their standard deviations $\sigma_{\left\langle z_{0}\right\rangle}$ for different optical density filters and both pinholes are presented in the Table 3.3 .

A small volume element $(\approx 1 f l)$ facilitates a fast exchange of fluorophores in and out the sample volume element. Due to the short passage times, higher excitation intensities can be applied without photobleaching the molecules, thus allowing higher count rates per molecule. Another important factor is the strong reduction in the background noise. The scattering is proportional to the sample volume, i.e. the number of solvent molecules and to the excitation intensity. When the excitation intensity is high, the background intensity consists predominantly of Raman scattering from the solvent. At lower excitation levels, the background count consists mainly of the dark current from the detectors $(\cong 200 \mathrm{cps})^{10}$ (see

\footnotetext{
${ }^{10} \mathrm{cps}=$ count per second
} 
Table 3.3: $\mathrm{b}$ The mean half length $\left\langle z_{0}\right\rangle$ of the focal volume obtained from particle number and concentration of Rhodamine $6 \mathrm{G}$ via mole number for $100 \mu \mathrm{m}$ and $30 \mu \mathrm{m}$ pinholes. $\sigma_{\left\langle z_{0}\right\rangle}$ is its standard deviations.

\begin{tabular}{|c|c|c|c|c|c|}
\hline $\begin{array}{c}\text { Pinhole } \\
\text { OD Filters }\end{array}$ & $\begin{array}{c}\text { Molecule } \\
\text { number }\end{array}$ & $\begin{array}{c}\text { Mole number } \\
\cdot 10^{-23}\end{array}$ & $\begin{array}{c}\text { Volume } \\
\cdot 10^{-18} \mathrm{~m}^{3}\end{array}$ & $\begin{array}{c}\left\langle z_{0}\right\rangle \\
\cdot 10^{-6} \mathrm{~m}\end{array}$ & $\begin{array}{c}\sigma_{\left\langle z_{0}\right\rangle} \\
\cdot 10^{-6} \mathrm{~m}\end{array}$ \\
\hline $100 \mu \mathrm{m} ; 2$ & 4.22 & 0.70 & 0.70 & 1.28 & 0.01 \\
\hline $100 \mu \mathrm{m} ; 1$ & 7.23 & 1.20 & 1.20 & 2.02 & 0.02 \\
\hline $100 \mu \mathrm{m} ; 0.6$ & 10.09 & 1.68 & 1.68 & 2.21 & 0.04 \\
\hline $100 \mu \mathrm{m} ; 0.2$ & 19.12 & 3.17 & 3.17 & 2.24 & 0.03 \\
\hline $30 \mu \mathrm{m} ; 2$ & 3.30 & "0.55 & 0.55 & 1.94 & "0.04 \\
\hline $30 \mu \mathrm{m} ; 1$ & 4.31 & 0.72 & 0.72 & 2.10 & 0.03 \\
\hline $30 \mu \mathrm{m} ; 0.6$ & 7.86 & 1.30 & 1.30 & 2.70 & 0.03 \\
\hline
\end{tabular}

Fig 3.4) and corresponds to about one thousands times less than the emission rate detectable from a single rhodamine molecule ( $\cong 1000 \mathrm{kcps}$ ).

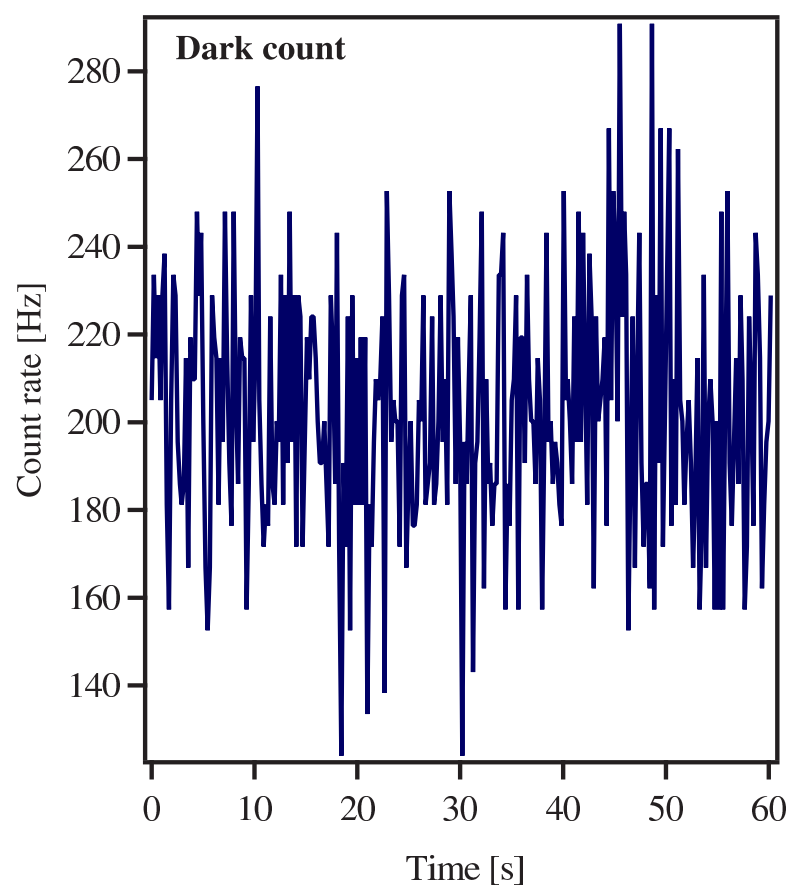

Figure 3.4: The dark count of the photodiodes.

\subsubsection{The excitation power of the laser}

The excitation power of the laser $P$ was measured with a Powermeter (Fieldmaster) from the company Coherent Model LM-2 (up to $50 \mathrm{~mW}$ ). The output power was measured as equal to $5.05 \mathrm{~mW}$. The measured excitation power of the laser and the percent of transmission are 
shown in Table 3.4. More than $60 \%$ of the laser light passes through our optical system

Table 3.4: The measured excitation power $\mathrm{P}$ of the laser.

\begin{tabular}{|c|c|c|c|c|c|}
\hline $\begin{array}{c}\text { OD } \\
\text { Filters }\end{array}$ & $\begin{array}{c}\text { After } \\
\text { OD }\end{array}$ & $\begin{array}{c}\text { After } \\
\text { the beamsplitter }\end{array}$ & $\begin{array}{c}\text { After } \\
\text { the mirror }\end{array}$ & $\begin{array}{c}\text { After } \\
\text { the objective }\end{array}$ & $\begin{array}{c}\text { Transmission } \\
\%\end{array}$ \\
\hline 3 & $3.94 \mu \mathrm{W}$ & $3.0 \mathrm{~W}$ & $2.91 \mu \mathrm{W}$ & $2.41 \mu \mathrm{W}$ & 61.2 \\
\hline 2 & $41.3 \mu \mathrm{W}$ & $31.2 \mu \mathrm{W}$ & $30.3 \mu \mathrm{W}$ & $25 \mu \mathrm{W}$ & 60.5 \\
\hline 1 & $406 \mu \mathrm{W}$ & $309 \mu \mathrm{W}$ & $300 \mu \mathrm{W}$ & $247 \mu \mathrm{W}$ & 60.8 \\
\hline 0.6 & $1.13 \mathrm{~mW}$ & $868 \mu \mathrm{W}$ & $839 \mu \mathrm{W}$ & $692 \mu \mathrm{W}$ & 61 \\
\hline 0.2 & $3.14 \mathrm{~mW}$ & $2.81 \mathrm{~mW}$ & $2.60 \mathrm{~mW}$ & $2.18 \mathrm{~mW}$ & 69 \\
\hline 0 & $4.47 \mathrm{~mW}$ & $3.48 \mathrm{~mW}$ & $3.38 \mathrm{~mW}$ & $2.78 \mathrm{~mW}$ & 62 \\
\hline
\end{tabular}

including the objective. The rest of the light is lost because some of it is partially reflected by the surface of lenses and optical system, some is lost in the imperfect transmission through optic components (absorption of the glues and coatings), and some is scattered by dust and surface imperfections on lenses and on the optical system. Really the actual power at the sample is lower still due to losses at the coverslip and water.

\subsubsection{FCS properties}

The photophysical and photochemical aspects of dyes are of great importance for the application of fluorescence correlation spectroscopy. For a high sensitivity it is required to have a high count rate per molecule and low background noise, i.e. a high signal-to-background ratio. It is possible to achieve this by using higher dye concentrations, resulting in lower intensity fluctuations, although longer measurement times are then necessary to analyse the lower amplitudes of the correlation curve. Nowadays one uses very small detection volumes, objectives with high apertures and highly selective band-pass filters. Nevertheless photophysical properties of the fluorescent label like its absorption coefficient, as well as its fluorescence, triplet and photobleaching quantum yields, are fundamental limits for the sensitivity and correct performance of FCS measurements [125].

A triplet state (see Figure 2.8 in Section 2.2.2) is a metastable state laying just below the lowest excited singlet state. It returns to the ground state by long-lived phosphorescence. In this case phosphorescence is an unwanted process which disturbs the FCS curve on a short time scale (see Figure 3.5 (a)). Oxygen is used to try to quench the triplet state, this being a very small molecule and therefore can penetrate into the membrane well, and it has a high, constant rate for quenching $\left(k \approx 10^{10}\right.$ litres $/($ mole $\left.\cdot s e c)\right)[15]$ (see Figure $\left.3.5(b)\right)$. The 
measurements were performed in fast crosscorrelation mode ${ }^{11}$. Various mechanisms for the transfer of excitation energy from the triplet state of a donor (a wide variety of dye molecules) to an acceptor (oxygen) have been suggested. The transfer occurs as a triplet-singlet transition in the donor and as the excitation of oxygen from its ground state to its low-lying singlet state or, as a singlet-triplet transition in oxygen to be relaxed. Oxygen can be involved in the formation of a charge-transfer complex with the excited molecule $\left(A^{*+} \cdot O_{2}^{-}\right)$, which releases the excitation energy through internal conversion processes [15]. The photochemical process in which triplet energy transfer occurs is called photoquenching. Another unwelcome

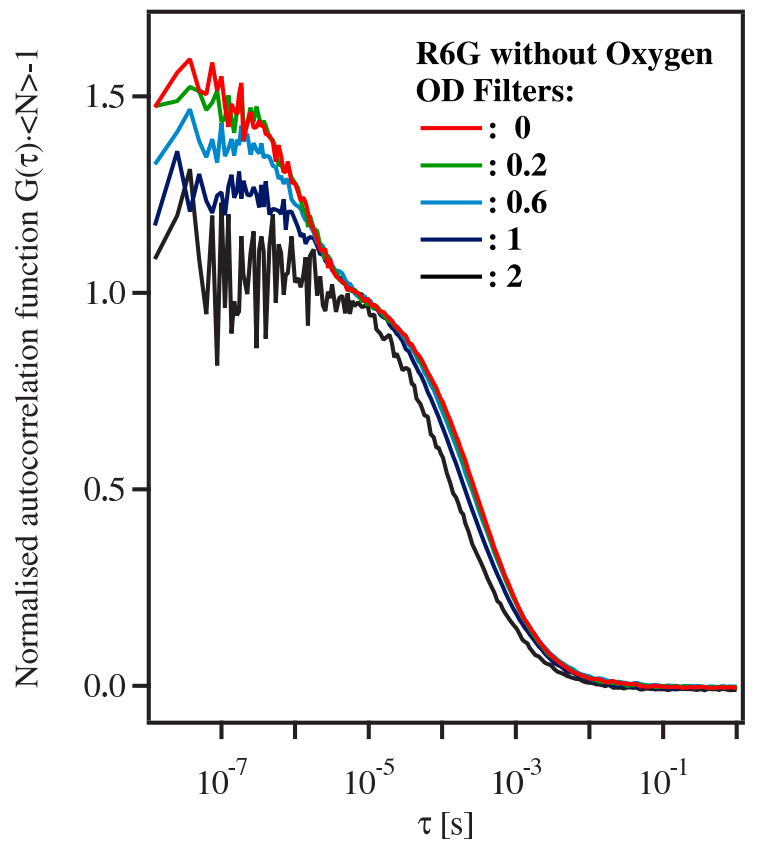

(a)

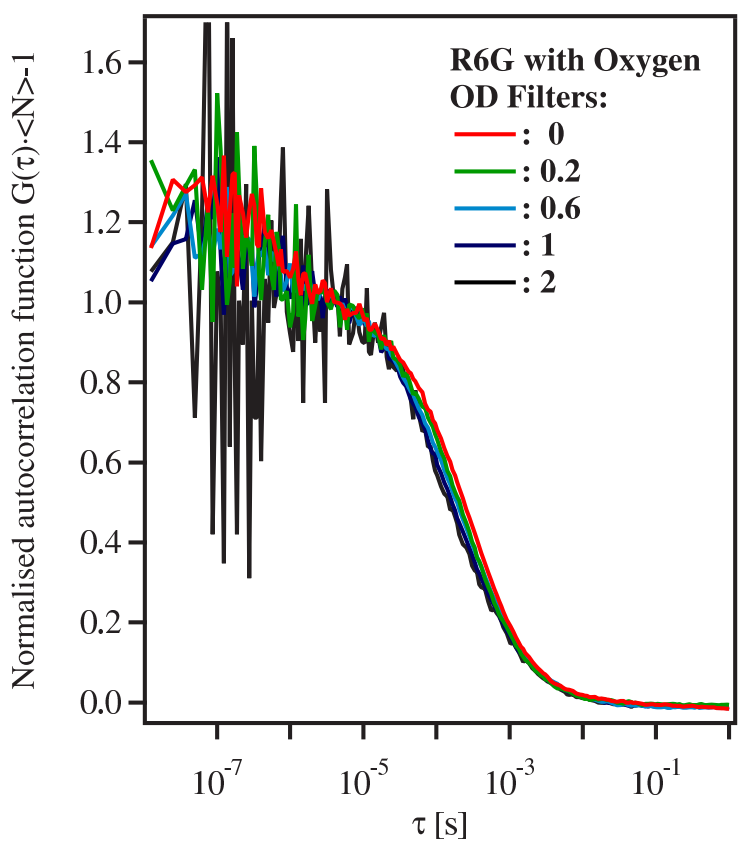

(b)

Figure 3.5: (a) FCS correlation curves of rhodamine 6G without oxygen. (b) FCS correlation curves of rhodamine $6 \mathrm{G}$ with oxygen as a quencher. All of measurements were performed with different laser power in Single Cross Fast mode (SCF).

side effect is quenching of the fluorescence. It occurs when dye molecules interact with each other thus forming complexes which release the excitation energy in a non-radiative way. It results in a lower fluorescence intensity and in a lower mean label molecule number.

One of the most important processes, that define the image quality and number of photons detected from the sample is photobleaching, this being an irreversible process of photo-

\footnotetext{
${ }^{11}$ Fast crosscorrelation mode means that the fluorescent signal from one avalanche photodiode (APD) at time $t$ is correlated with the signal from the same one at time $t+\tau$, where $\tau$ is very small thus resulting in correlation over fast time scale.
} 
degradation of a fluorescent molecule. It occurs at higher excitation intensities, where the excitation rate is on the same level as the decay of the excited singlet states of labels. Then the excited singlet state is saturated such that the molecule can never come back to the ground state by fluorescence. The saturation is observed at higher laser power (see Table 3.5, ODs 0.2 and 0), resulting in decreasing count rate per molecule (see Figure 3.5). Photobleaching is related to the triplet state, the probability of which increases with increasing excitation irradiance. The triplet state releases its excitation energy in non-radiative processes: intersystem crossing (ISC) and internal conversion (IC) which are assumed to be photobleaching reactions. Photoblinking is a case of a reversible photo-degradation, where a dye particle, can after a while, emit fluorescent light again.

The level of photobleaching has been determined according to Eggeling [124]. The calculated radius of the laser focus from rhodamine $6 \mathrm{G}$ measurements was the same for both photodiodes $\mathrm{A}$ and $\mathrm{B}(\mathrm{rA}=\mathrm{rB})$. The area in the plane of focus is:

$$
S=\pi \cdot r^{2}
$$

The focal excitation irradiance is [25]:

$$
I_{0}=\frac{P}{0.5 \cdot \pi \cdot r^{2}}
$$

where $P$ is the measured laser power.

The power of the laser per $\mathrm{cm}^{2}$ in focus is expressed by Eq. 3.8 and its values are shown in Table 3.5:

$$
\frac{I_{0}}{2}=\frac{P}{\pi \cdot r^{2}} \quad\left(\frac{W}{c m^{2}}\right)
$$

The probability of photobleaching at each excitation event for Rhodamine R6G is a constant level of $1.2 \cdot 10^{-6}$ for the laser power below $10^{4} \mathrm{~W} / \mathrm{cm}^{2}$ (refer to Figure 3.6) [25]. Increasing the laser intensity above $10^{4} \mathrm{~W} / \mathrm{cm}^{2}$ results in a decreasing count rate per molecule, especially for the lower density filters e.g. 0.2 and 0 . This means that for such high intensities, photobleaching and saturation of labels occur and consequently it was decided to use a filter with an optical density of 1 for the R6G measurements. The diffusion time of Rhodamine through $377 \mathrm{~nm}$ radius of the focus is approximately equal to $100 \mu$ s for pinhole $100 \mu \mathrm{m}$. The diffusion time of a label in a fluid membrane is about $10 \mathrm{~ms}$ and in a gel membrane about $200 \mathrm{~ms}$. So the label in fluid membranes needs 100 times more time for diffusing through the 
Table 3.5: The irradiance in the focus.

\begin{tabular}{|c|c|c|c|c|c|}
\hline $\begin{array}{c}\text { OD } \\
\text { Filters }\end{array}$ & $\begin{array}{c}\text { Molecules } \\
\text { number }\end{array}$ & $\begin{array}{c}\text { Count } \\
\text { rate, } k H z\end{array}$ & $\begin{array}{c}\text { Radius } \\
\cdot 10^{-5} \mathrm{~cm}\end{array}$ & $\begin{array}{c}\mathrm{I}_{0} / 2 \\
\cdot 10^{4} \frac{\mathrm{W}}{\mathrm{cm}^{2}}\end{array}$ & $\begin{array}{c}\text { Count rate } \\
\text { per molecule, } k H z\end{array}$ \\
\hline 3 & 7.29 & 4.3 & 4.13 & 0.022 & 0.59 \\
\hline 2 & 4.22 & 25 & 3.62 & 0.304 & 5.92 \\
\hline 1 & 7.23 & 172 & 3.77 & 2.767 & 23.79 \\
\hline 0.6 & 10.09 & 241 & 4.28 & 6.015 & 23.89 \\
\hline 0.2 & 19.12 & 366 & 5.81 & 10.28 & 19.14 \\
\hline 0 & 17.57 & 317 & 5.91 & 12.67 & 18.04 \\
\hline
\end{tabular}

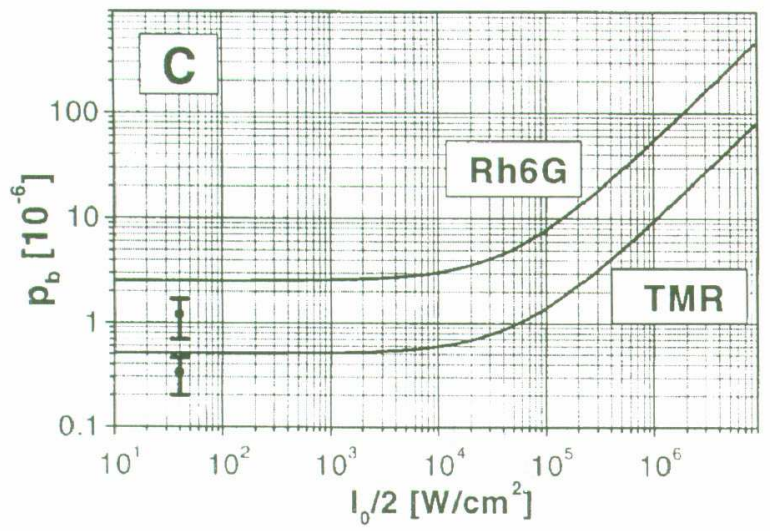

Figure 3.6: Probability of photobleaching for Rhodamine R6G [25].

laser focus than a dye in solution (in gel membranes 2000 times more time). It means, that for keeping photobleaching processes on a neglible level, one should reduce the power of the laser (by 100 times in fluid membranes and 2000 times in gel membranes) by using higher OD filters, e.g. OD 3, when measuring membranes. This is a rough approximation, because the probability of photobleaching differs for various labels.

Another important parameter for single molecule detection is signal-to-background ratio [27]. The background is composed of scattered laser light, Raman scattering from the solvent, as well as of the own solvent and optical fluorescence. The Raman scatering from water was blocked with band-pass fluorescent filters (refer to Section 3.1.1). Signal-to-background ratios are kept above a limit of 1000:1 for rhodamine $6 \mathrm{G}$ in solution. 


\subsection{Membrane preparation}

Hydrated, multilamellar membranes of mixtures of various phospholipids (diacyl phosphatidylocholines) with different chain lengths were investigated. Membranes were supported on quartz coverslips (Firma Plano ${ }^{12}$ ) or on glass coverslips. The lipids were purchased from Avanti Polar Lipids, Inc ${ }^{13}$ :

- DLPC: 12:0-12:0 1,2-dilauroyl-sn- glycero-3-phosphocholine,

- DMPC: 14:0-14:0 1,2-dimyristoyl-sn-Glycero-3-phosphocholine,

- DPPC: 16:0-16:0 1,2-dipalmitoyl-sn-glycero-3-phosphocholine,

- DSPC: 18:0-18:0 1,2-distearoyl-sn-glycero-3-phosphocholine,

- POPC: 16:0-18:1 PC, 1-palmitoyl-2-oleoyl-sn-glycero-3-phosphocholine.

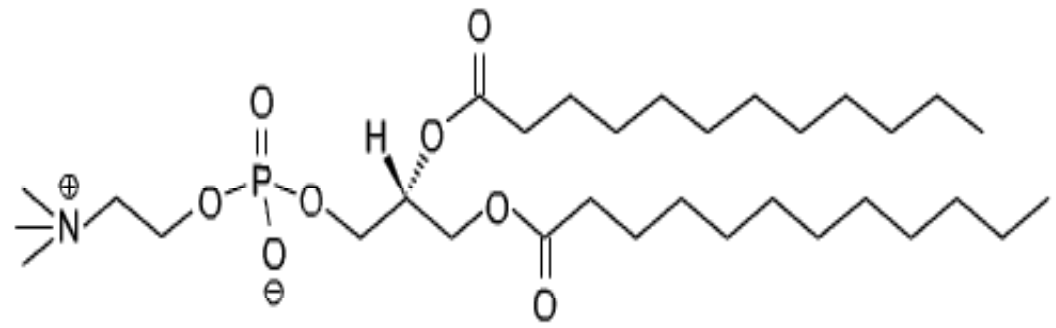

Figure 3.7: An example of the chemical structure of DLPC.

DLPC, DMPC, DPPC, DSPC are saturated phospholipids, where POPC is an unsaturated phospholipid with one double $C=C$ bond in the longer chain. As a label we have used one of the following fluorescent lipid analogues: TRITC DHPE, DiIC18 and BODIPY-C12-HPE. Before the sample preparation, the quartz glass was washed with a solution of 2:1 Dichloromethane : Methanol, then in an ultrasound bath with acetone and afterwards in bidistilled water. Firstly lipids and labels were dissolved separately in Dichloromethane : Methanol (2:1) or TFE (2,2,2 - Trifluoroethanol). The solvent TFE has the advantage that it reduces surface tension and a drop of lipid solutions lies flatter on a slide. In the case of FCS experiments, $1 \mathrm{ml}$ of $10 \mathrm{mM}$ lipid mixture was mixed with a $25 \mu \mathrm{l}$ of a $10 \mathrm{nM}$ dye solution.

${ }^{12}$ Wetzlar, Germany

${ }^{13}$ Alabaster, AL, USA. 
Fluorescent labels were purchased from Molecular Probes (Leiden, The Netherlands). The ratio of lipid molecules to fluorescent label molecules is $1: 4 \cdot 10^{7}$, resulting in only a few dye molecules being in a focus during an FCS experiment. For single molecule measurements $1 \mathrm{ml}$ of $10 \mathrm{mM}$ lipid solution was mixed with a $5 \mu \mathrm{l}$ of $10 \mathrm{nM}$ dye solution, which makes the ratio of lipid molecules to fluorescent labels molecules equal to $2 \cdot 10^{8}$. Then two $10 \mu \mathrm{l}$ drops of this solution was put on a quartz glass (or on a glass coverslip in the case of the TFE solvent). The solvent was evaporated under a stream of nitrogen and left for some hours in a desiccator (vacuum) in order the rest of the organic solvent was removed. A drop of bidistilled water $(30 \mu \mathrm{l})$, saturated with oxygen, was put on the dried lipid and the sample was heated at a temperature above the melting point for approximately 2 minutes. Oxygen reduces the noise on fast time scales because it quenches the triplet state (see Section 3.1.2.). The loss of water during experiments because of heating was thus compensated. If the sample was dried out accidentally, a new one was substituted immediately.

In the present of water, lipid bilayers are formed, these being approximately $5 \mathrm{~nm}$ in thickness and there is an aqueous layer of approximately $2 \mathrm{~nm}$ thickness as in Figure 3.8, [84; 68]. In the case presented here many multilamellars (stacks) were used. The formation of stacks is well known from FCS experiments via the mean number of days in the focus ${ }^{14}$, as well as from neutron and X-ray scattering experiments [91]. The sample was covered with a second coverslip in order to avoid increasing the thickness of a water layer, which might result in vesicles forming as well as bilayers swimming in and out of focus. Using a stock of bilayers allows to avoid an influence of a quartz on diffusion processes in membranes. It will influence diffusion mainly in the first monolayer, therefore the mean number bilayers into focus in the range of 10 to 100 what in that case the influence of the quartz support can be neglected.

Temperature can be changed in this setup. For this purpose water was heated or cooled in a water bath (RKS 20, ggw $^{15}$ ), and pumped through a heating/cooling system. This system consists of specially constructed sleeves placed around the objective and the sample. However the pumping system was switched off during the measurement periods (120-300 s), because it was a source of mechanical vibrations that would otherwise have disturbed the measurement. Temperatures were measured in a drop of water on a covering coverslip with an ultra-thin thermocouple in the beginning and the end of measurements. The difference in

\footnotetext{
${ }^{14}$ The concentration of lipids and labels is known, so the ratio of lipid molecules to labels is known also. From FCS the mean number of dyes (from the amplitude of correlation function) and the size of the focus is computed. From this information the mean number of bilayers can be established.

${ }^{15}$ Lauda, Germany.
} 


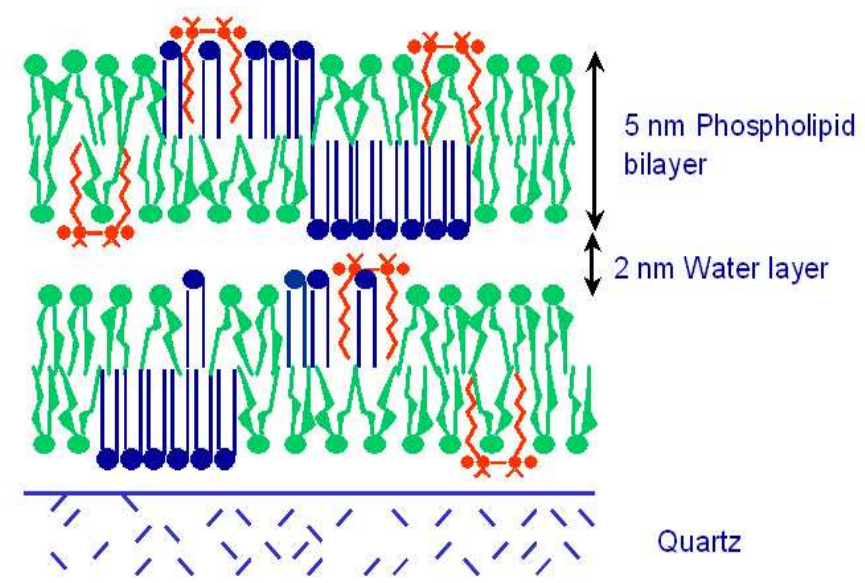

Figure 3.8: Schema of a stack of labelled bilayers supported on a quartz coverslip. The green colours corresponds to lipids in a fluid state, the blue ones in a gel state, and the red colours represents a DiI label.

temperatures between beginning and end of measurement period was typically $\pm 0.2 \mathrm{~K}$ (with a measured maximum change of $\pm 0.5 \mathrm{~K}$ ).

\subsubsection{Label properties}

The fluorescent signal comes from a dye diffusing through the laser focus. Different labels with absorption maxima lying as close as possible to the $532 \mathrm{~nm}$ wavelength of the laser were used. These large polyatomic organic molecules dissolved in solution have broad absorption and emission spectra due to the large number of vibrational transitions (refer to Figure 3.11). Such a broad spectrum has the advantage that even when the absorption maximum and the laser wavelengths differ for some nanomiters, it is still possible to have a high probability of absorption of a photon. As diffusing particles in the membranes one of the following fluorescent lipid analogues, purchased from Molecular Probes ${ }^{16}$ were used:

- T-1391: TRITC DHPE; N-(6- tetramethylrhodaminethiocarbamoyl)-1,2- dihexadecanoylsn-glycero-3- phosphoethanolamine, triethylammonium salt. This has a fluorescent particle connected to the head and carries a negative charge in the head group (see Figure 3.9). It has an absorption maximum at $540 \mathrm{~nm}$ and an emission maximum at $566 \mathrm{~nm}$.

- DiI dyes: D-384: DiIC16; 1,1'-dihexadecyl-3,3,3',3'- tetramethylindocarbocyanine

\footnotetext{
${ }^{16}$ Alabaster, AL, USA.
} 


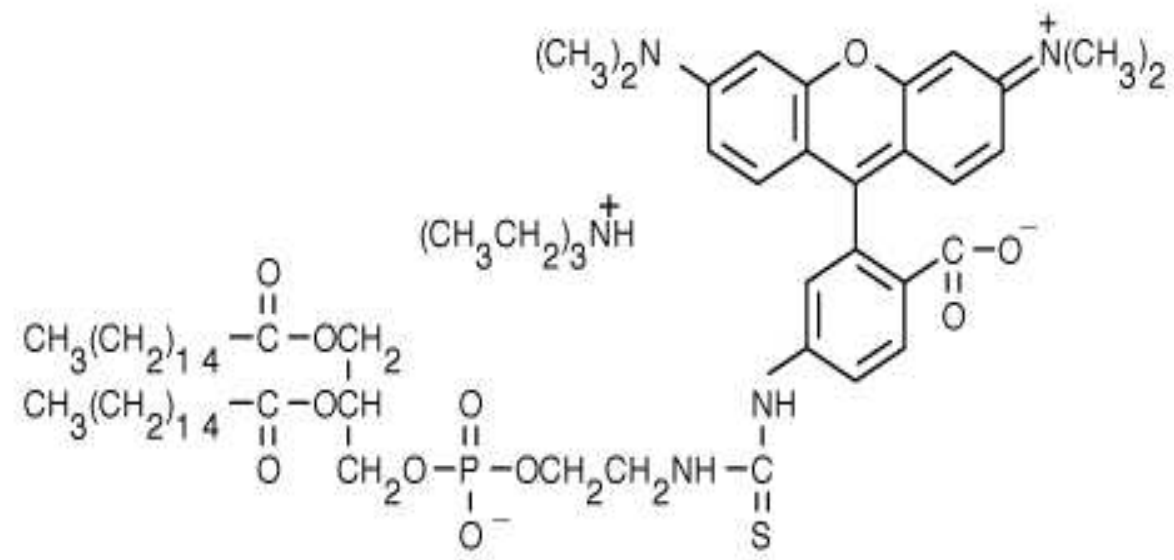

Figure 3.9: The chemical structure of TRITC DHPE.

perchlorate and D-282: DiIC18; 1,1'-dioctadecyl-3,3,3',3'- tetramethylindocarbocyanine perchlorate. They have a fluorescent particle built into the head group (refer to Figure 3.10). They have the same photochemical properties, but they differ in chain length for two carbon atoms. They both absorb strongly at $549 \mathrm{~nm}$ and have emission maxima at $565 \mathrm{~nm}$ (see Figure 3.11 (a)). DiI dyes incorporate a lipid molecule between their chains thus they are more likely to mimic the properties of natural lipids. They might also be used for rotation measurements of single lipids.

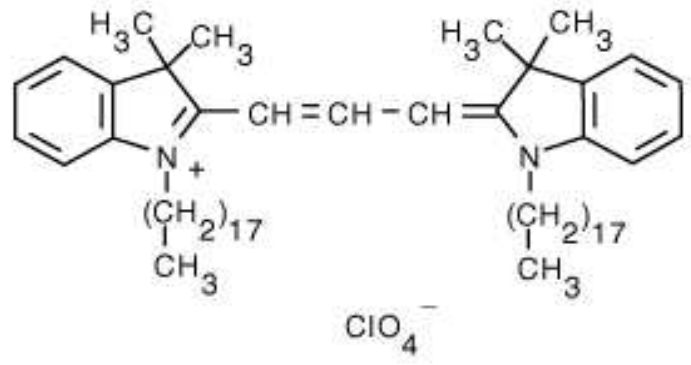

Figure 3.10: The chemical structure of DiIC18.

- D-3813: $\beta$-BODIPY(r) 530/550 C12-HPE; 2-(4,4-difluoro-5,7-diphenyl-4-bora-3a, 4adiaza-s-indacene-3-dodecanoyl)-1-hexadecanoyl-sn-glycero-3- phosphoethanolamine, which has a fluorescently labeled acyl chain (see Figure 3.12). BODIPY has an absorption maximum at $534 \mathrm{~nm}$ and an emission maximum at $551 \mathrm{~nm}$ (see Figure 3.11 (b)). 


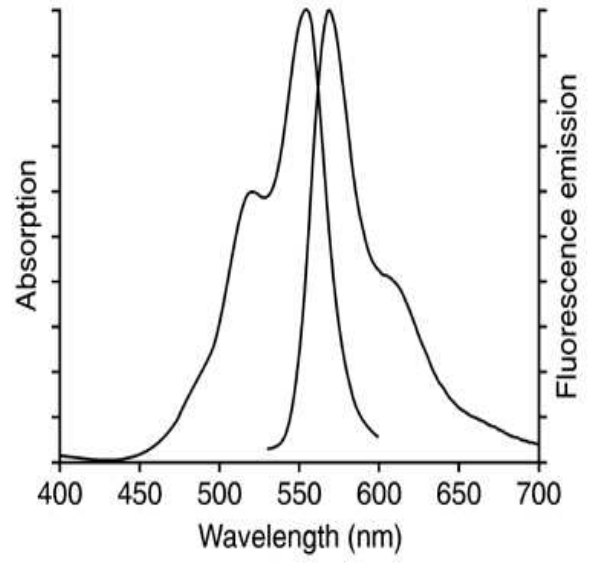

(a)

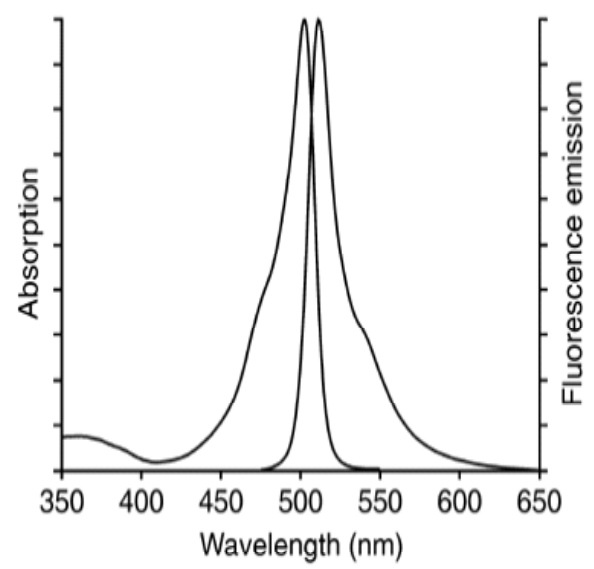

(b)

Figure 3.11: An example of an absorption (left curve) and an emission (right curve) spectra for (a) DiIC18 and for (b) $\beta$-BODIPY C12-HPE.

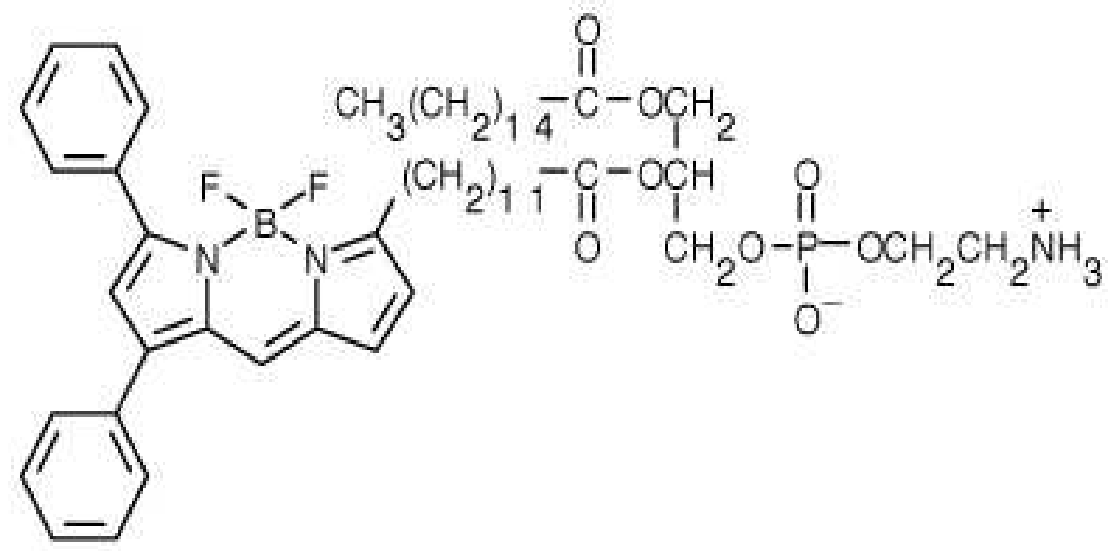

Figure 3.12: The chemical structure of BODIPY C12-HPE.

D-3813 is intrinsically lipophilic, unlike most other long-wavelength dyes. BODIPY fluorophores have a high photostability, strong absorbance and are largely insensitive to their environment, but in high concentrations aggregation may result in self-quenching. It has the advantages that it does not carry any charges, so it can be used for salt measurements, nor will it interact with the ions.

The purpose of this work was to investigate diffusion behavior in membranes and the label has therefore to be distributed randomly throughout the whole system. Therefore calorimetric 
studies has been performed to find out the optimal label which will partition equally well in a gel and in a fluid phase. For these studies, $5 \mathrm{mM}$ lipid solutions were mixed with various dyes separately so those mixtures contain $1 \%$ labels. The transition enthalpy was corrected for a $10 \mathrm{nM}$ lipid solution. The calorimetric melting profiles for DLPC, DPPC, DMPC, DSPC multilamellar vesicles with dyes obtained at a scan rate of $5 \mathrm{~K} / \mathrm{hr}$ are shown in Figure 3.13. The same measurements were then repeated at a scan rate of $1 \mathrm{~K} / \mathrm{hr}$ over a shorter temperature range. DLPC has a melting temperature just below the freezing point of water, therefore it has been measured in a capillary cell with a smaller volume immersed in a 40:60 ethylenglycol:water mass \% solution. Since it has a very broad transition it was difficult to see the influence of a label.

In the presence of various dyes, the maxima of the heat capacity curves are shifted progressively to lower temperatures. This indicates that these labels prefer fluid-like domains. The partitioning into a fluid or a gel phase depends on the relative chain lengths of the label acyl chains and the phospholipids acyl chains. When the dye chain lengths are close those of the lipids, packing is efficient and thus stronger interchain interactions in the gel provides favourable partitioning into that phase. However the chain length is not the only parameter for maximum partitioning into the gel phase. That is why it is necessary to perform calorimetric measurements in order to know the partitioning of labels into gel and fluid domains. The dye DiIC18 seems to have almost the same distribution in a fluid phase as well as in a gel one in DMPC and DSPC membranes, this being found also by Feigenson [108]. TRITC DHPE prefers slightly a fluid phase in the mixture DLPC:DPPC, although it has been used here because of its high photostability. 


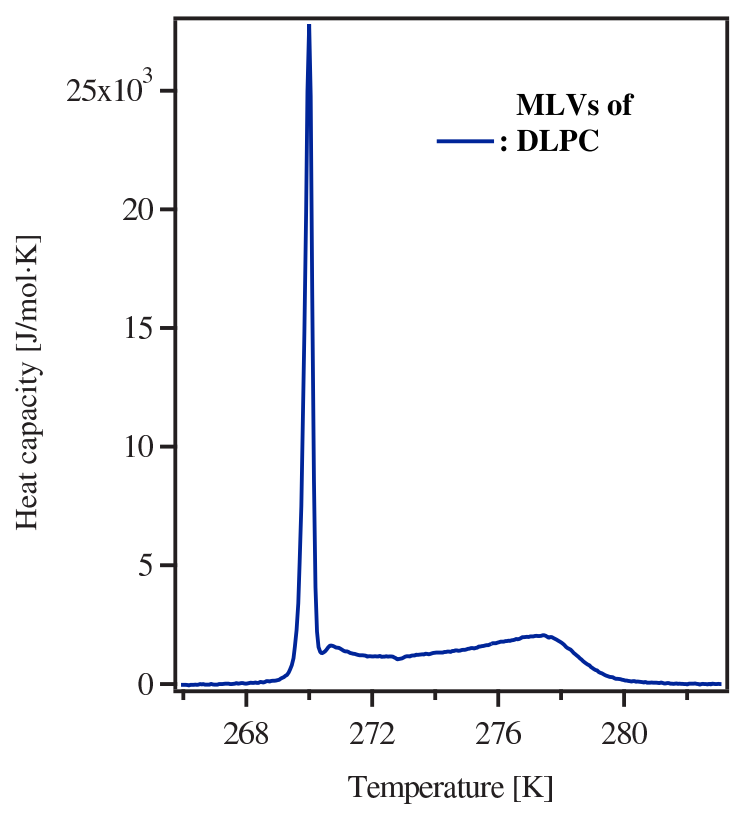

(a)

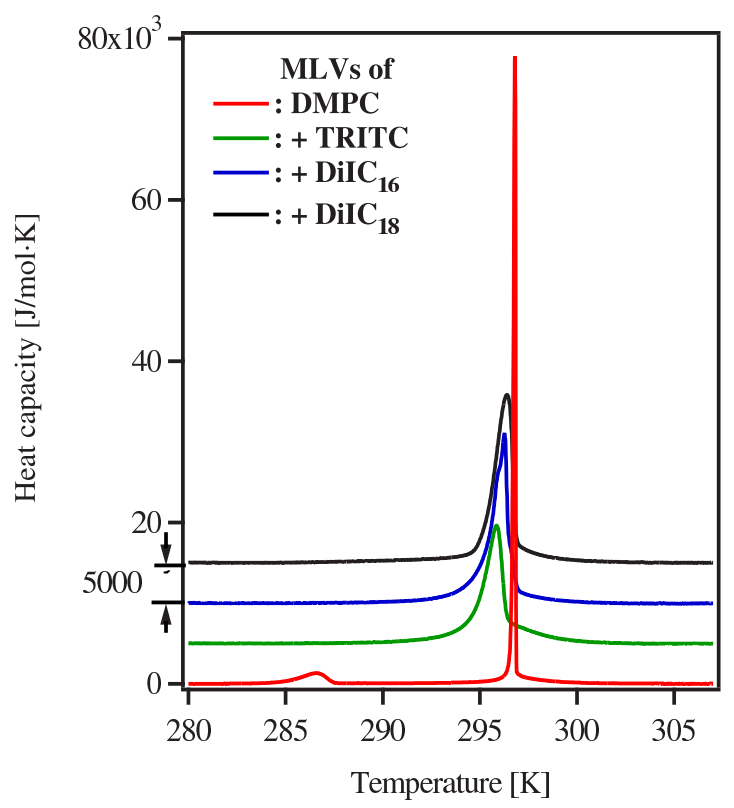

(c)

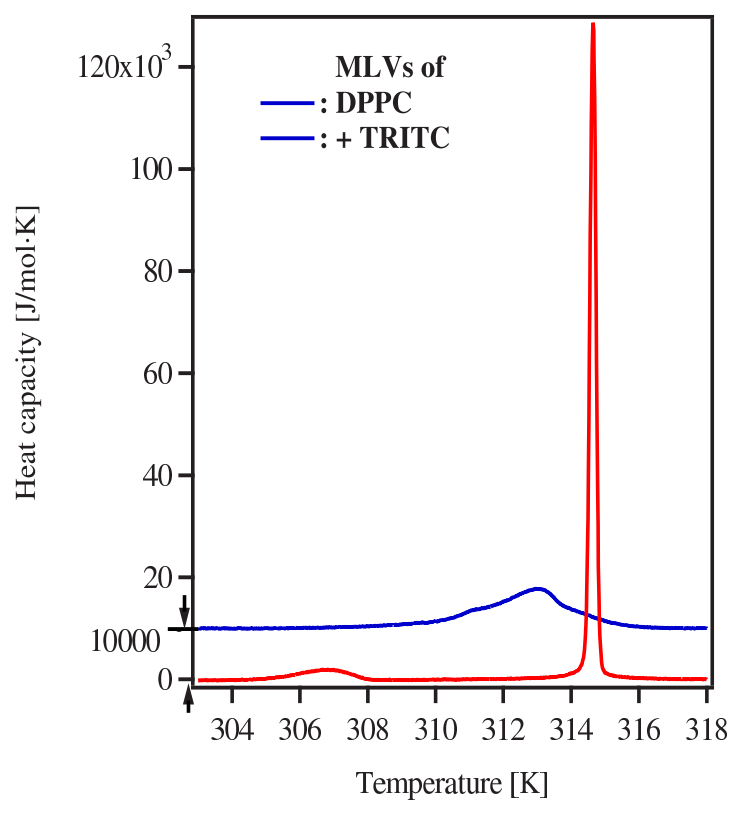

(b)

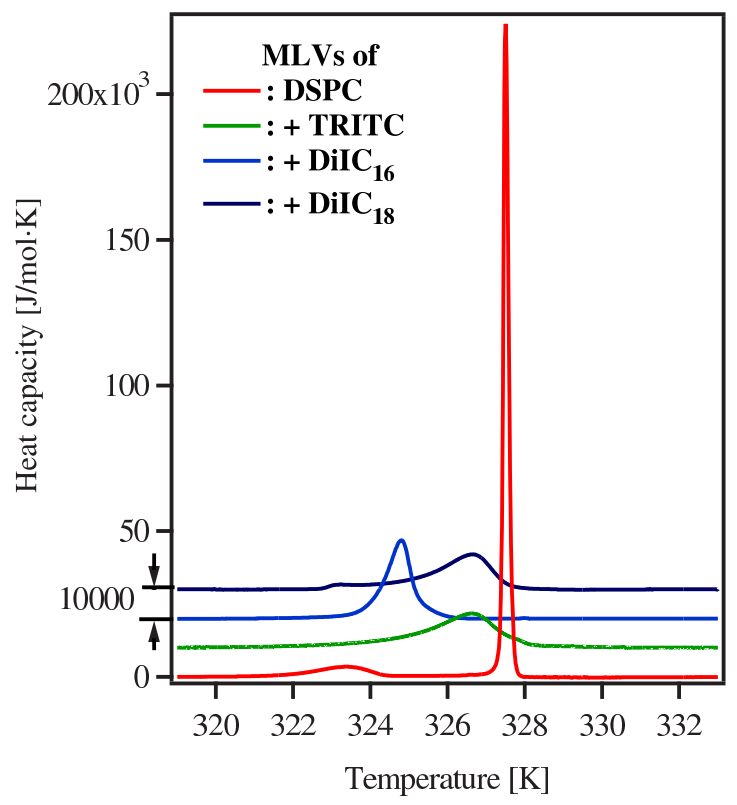

(d)

Figure 3.13: (a) Heat capacity curves of MLVs of DLPC. (b) Heat capacity curves of MLVs of DPPC with $1 \%$ of labels. (c) Heat capacity curves of MLVs of DMPC with $1 \%$ of labels. (d) Heat capacity curves of MLVs of DSPC with $1 \%$ of labels. In both cases, the scan rate was $5 \mathrm{~K} / \mathrm{hr}$. 


\section{Chapter 4}

\section{Results}

This section presents the results of diffusion measurements using FCS and their interpretation with the help of Monte Carlo simulations.

The diffusion processes in artificial lipid membranes at various temperatures were investigated using Fluorescence Correlation Spectroscopy (FCS). Our systems consist of hydrated multilamellar planar membranes supported on coverslips made from mixtures of various phospholipids with different chain lengths (DLPC:DPPC, DMPC:DSPC ${ }^{1}$ ). Approximately 20 measurements of time length 120-300 s were measured and then averaged. Time scales were calibrated with a Rhodamine R6G (10 nM) solution at $296 \mathrm{~K}$ with a known diffusion constant of $3 \cdot 10^{-6} \mathrm{~cm}^{2} / \mathrm{s}$ [33]. Most of the measurements on membranes were recorded with a $100 \mu \mathrm{m}$ pinhole and optical density (OD) filter 3 which reduces the irradiance in a focus to $0.022 \cdot 10^{-4} \mathrm{~W} / \mathrm{cm}^{2}$ in either crosscorrelation or autocorrelation mode. Crosscorrelation mode means that the fluorescent signal from one avalanche photodiode (APD), which detects parallel polarised fluorescent light at the time $t$, is correlated with the signal from the second APD, which detects perpendicular polarised fluorescent light, at time $t+\tau$. This method of correlation results in a reduction of the electronical noise of both photodiodes. Autocorrelation mode means that the fluorescent signal from one APD at the time $t$ is autocorrelated with the signal from the same APD at the time $t+\tau$. In the case of autocorrelation mode, there are much more electronic artifacts on fast time scales due to the dead time of the photodiode (30 ns). The data were analysed using a software written by the author. This software calculates the diffusion time and the focal radius for rhodamine $6 \mathrm{G}$ measurements and the diffusion time and diffusion coefficient in the case of membranes.

${ }^{1}$ DLPC: 12:0-12:0 1,2-dilauroyl-sn- glycero-3-phosphocholine, DMPC: 14:0-14:0 1,2-dimyristoyl-sn-Glycero-3-phosphocholine, DPPC: 16:0-16:0 1,2-dipalmitoyl-sn-glycero-3-phosphocholine,

DSPC: 18:0-18:0 1,2-distearoyl-sn-glycero-3-phosphocholine, 


\subsection{Single molecule measurements}

For detection of a single molecule (SM), the ratio of lipid molecules to fluorescent label molecules was reduced to $2 \cdot 10^{8}$. This results in less than two dye molecules in the detected volume of $\sim 0.7 \mathrm{fl}$ (on average, 1.7 molecules in the fluid phase and 1.65 molecules in the gel phase). The average number of molecules was established as being equal to the inverse of the amplitude of the correlation function at a correlation time of zero. This study was performed on the stack of DMPC membranes labelled with TRITC DHPE ${ }^{2}$ at two different temperatures: one at which the lipids were in the fluid phase $(301 \mathrm{~K})$ and one at which the lipids were in the gel phase $(282 \mathrm{~K})$. The diluted rhodamine solution had a concentration of $5 \mathrm{nM}$ and it was measured at $296 \mathrm{~K}$. These measurements were recorded at a bin width ${ }^{3}$ of $10 \mu$ s with $10^{5}$ intervals and $100 \mu$ s with $10^{4}$ intervals, so each measurement was $1 \mathrm{~s}$ long. Afterwards, the fluorescent traces for the rhodamine $6 \mathrm{G}$ and for fluid and gel domains were integrated with different bin widths. The count rate per single molecule ranged from 1.2 to $2 \mathrm{kHz}$ with OD filter 3 ( $\approx 22$ to $26 \mathrm{kHz}$ for rhodamine $6 \mathrm{G}$ with OD filter 1 ). The background noise from water was equal to $500 \mathrm{~Hz}$ with OD filter 3 and $2 \mathrm{kHz}$ with OD filter 1 (refer to Figure 4.1). Count rates differed by a factor of four although there a factor of one hundred in filter transmission exists. This means that the fluorescent band-pass filters does not totally exclude the additional scattered laser light on optics, Raman scattering from the water and optical fluorescence.

The fluorescent intensity fluctuations were performed in two ways. Firstly the detected photons were registered by a multichannel counter (CIO-CTR05 $5^{4}$ ). With this multichannel counter it is possible to detect each single photon separately. The stored data were analysed later. The fluorescent trace of a marker reflects the environment of the label within a given domain. In Figure 4.2 the fluorescent light intensity fluctuations coming out from very fast diffusing soluble molecules in water can be seen along slower diffusing labels in fluid membranes and very slow diffusion in gel membranes. The diffusion time was established by the duration of the burst which, from definition, is the number of detected photons associated with a transit of a single molecule through the probe volume. Since a lot of randomly diffusing molecules cross only the edges of the detection volume, a short diffusion time results. Also only a few molecules traverse the centre of the focal volume. One can further see this on

\footnotetext{
${ }^{2} \mathrm{~T}-1391, \quad \mathrm{~N}-(6-\quad$ tetramethylrhodaminethiocarbamoyl)-1,2- dihexadecanoyl-sn-glycero-3- phosphoethanolamine, triethylammonium salt.

${ }^{3}$ The bin width is the integration time for a single photon.

${ }^{4}$ PLUG-IN Electronic GMBH, Eichenau, Germany.
} 
the fluorescent traces as detected photons at both shorter and longer times. Single molecule events that have a long pathway through the focal volume were chosen in order to compare these results with the average diffusion times detected using a FLEX 5000/FAST correlator card from the FCS setup. This correlator card has the advantage that the fluorescent signal is correlated online, but the disadvantage is that it stores the fluorescent events integrated over $100 \mu$ s so one can not see the real fluorescent traces.

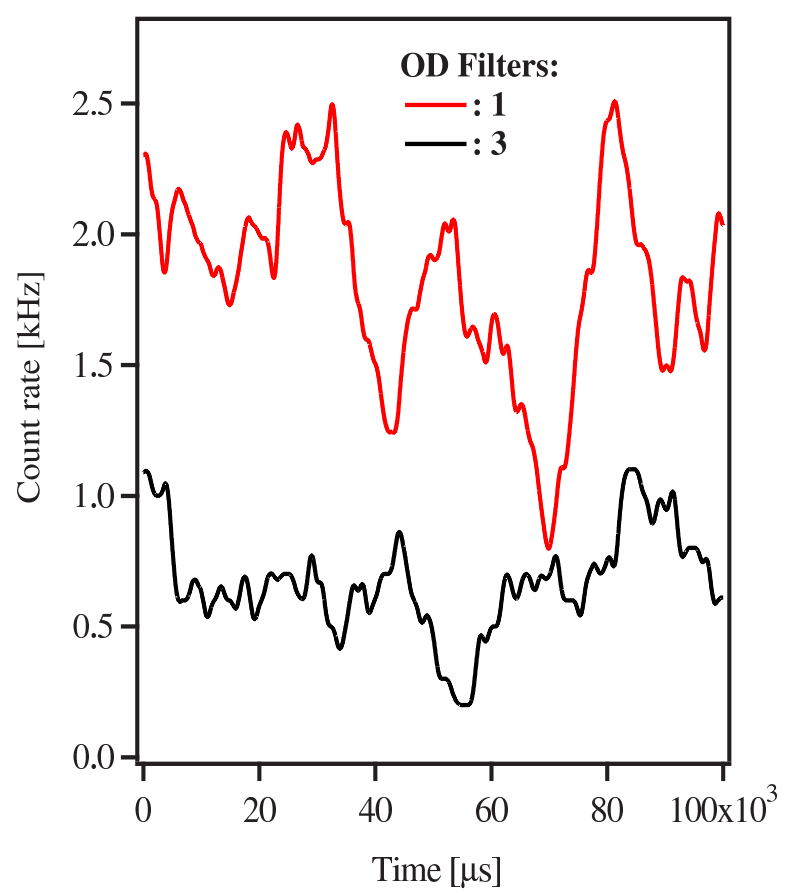

Figure 4.1: The background noise from water for OD 1 and OD 3 recorded with a multichannel counter. The fluorescence traces were integrated with a bin width of $10^{4}$ intervals.

Simultaneously correlation functions in crosscorrelation mode were recorded and calculated. With FCS, one can also distinguish between the time scales of diffusion processes. The mobility of dye strongly depends on the environment. The half-value decay time of these curves determine a diffusion time. Its value varies by several orders of magnitude between the small rhodamine 6G $(108 \mu \mathrm{s})$ molecule in water and the large dye molecule in gel membranes (211 ms, see Figure 4.3). The corresponding diffusion coefficients can also be calculated.

The results obtained with a multichannel counter are consistent with the results obtained with the correlator card. In contrast to the Flex card, the multichannel counter detects each single photon separately. Thus one can see the different movements of a single molecule in gel and fluid domains. However it is time consuming to record a lot of fluorescent traces in 


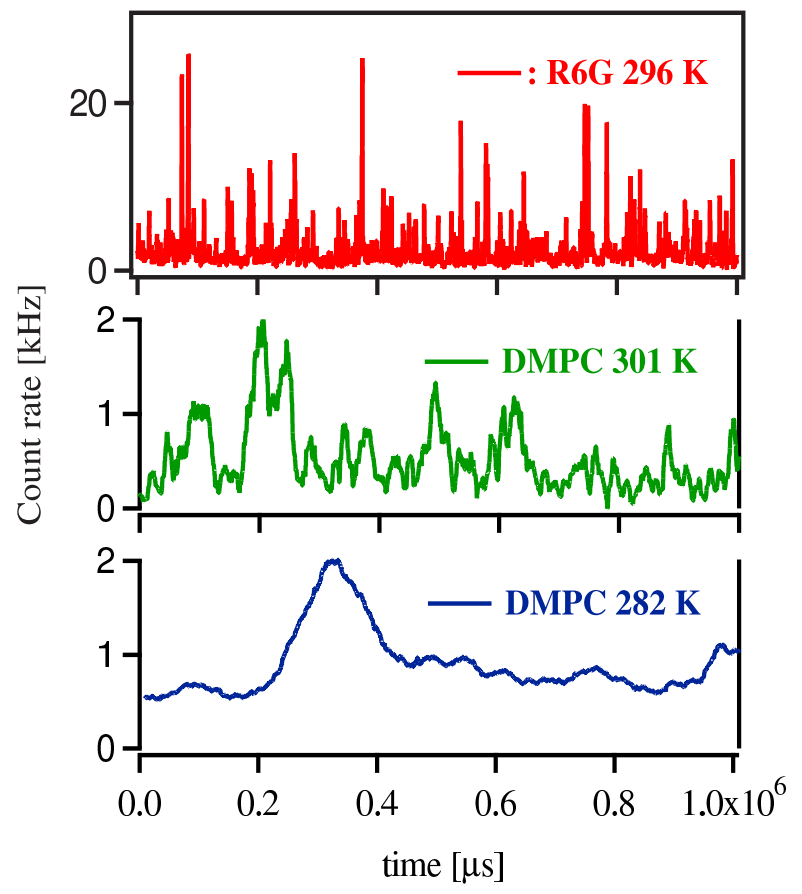

Figure 4.2: The fluorescent traces of rhodamine 6G diffusing in water (OD 1) and a dye TRITC DHPE diffusing in fluid and gel DMPC membranes (OD 3) recorded with a multichannel counter and then integrated with different bin widths.

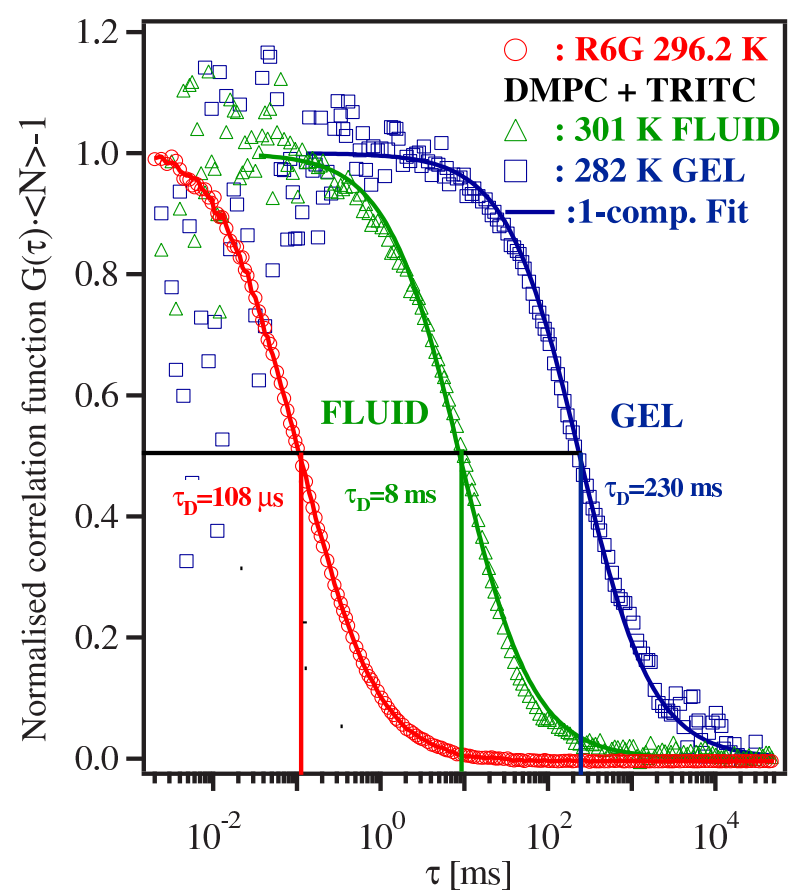

Figure 4.3: The corresponding normalised correlation function for rhodamine 6G diffusing in water (OD 1) and the dye TRITC DHPE diffusing in fluid and gel DMPC membranes (OD 3) calculated with a correlator card. 
order to have sufficient data points for statistical analysis. Although the correlator card has the advantage that the fluorescent signal is correlated online, the disadvantage is that it stores the fluorescent events integrated over $100 \mu$ s so one can not see the real fluorescent traces. However, with both cards one can study extremely small fractions of fluorescent labels and the time scales of diffusion processes.

\subsection{Domain visualisation}

\subsubsection{The phase diagram}

Heat capacity profiles of various DLPC:DPPC and DMPC:DSPC mole fractions allow the creation of phase diagrams which depict interactions between adjacent lipids. These interactions are expressed by the cooperativity parameter $\omega_{i j}^{m n}$. The highest value, $\omega_{12}^{l g}=$ $410 \mathrm{cal} /(\mathrm{mol} \cdot$ lipidchain), is for interactions between one molecule of DMPC in a fluid state and one DSPC molecule in a gel sate. The smallest value, $\omega_{12}^{l l}=60 \mathrm{cal} /$ ( mol - lipid chain), is for fluid-fluid interactions and $\omega_{12}^{g g}=145 \mathrm{cal} /(\mathrm{mol} \cdot$ lipid chain), for gel-gel interactions between DMPC and DSPC lipid molecules. The other cooperativity parameters have values between $\omega_{12}^{l g}$ and $\omega_{12}^{g g}$ (refer to Table 2.1). These values differ due to a difference in chain length. Mouritsen explains these parameters by "hydrophobic mismatch" which depicts the interaction of hydrophobic lipid tails with water when near neighbour molecules have different chains length. "Hydrophobic mismatch" suggests that the highest value of the cooperativity parameter, $\omega_{i j}^{m n}$, occurs for interactions of short fluid-like DMPC chains with long gel-like DSPC chains.

Differential scanning calorimetry (DSC) measurements for DMPC:DSPC lipid mixtures were performed by Schlötzer of the Membrane Thermodynamics and Biophysics Group of MPI for biophysical Chemistry [99]. In going from lower temperatures to higher temperatures, the lipid mixture starts to melt at the certain temperature. The lipids undergo a transition from a gel to a fluid state. There are two temperatures which determine the lower and upper end of transition and there is a region with a coexistence of gel and fluid phases (see Figure $4.4 \mathrm{a}$ ). The lower end of transition represents a temperature at which mainly the lipids, with a lower melting temperature (shorter acid chains), are melted. The upper end of transition represents mainly the melting of lipids with a longer acid chain length since those with a shorter chain length are melted earlier. Usually, one establish the phase limit as the intersection of the tangent of the $c_{p}$ profile with a base line [76]. These temperature points from various mixtures 
are used to construct the phase diagram.

A phase diagram is proposed by the regular solution theory which assumes macroscopic phase separation. In that case, heat capacity profiles show sharp defined transition temperatures (refer to Figure 4.4 b). However, very often there is no macroscopic phase separation when the real lipid systems melt. Lipid mixtures show a second order transition. The continuous nature of the melting causes the lateral separation of lipids in different states into microand macroscopic domains as discussed in Section 4.2.2 and by Mouritson in [81]. The "tangent approximation" makes the real $c_{p}$ profiles closer to the regular solution heat capacity profiles. Even finding the phase limits precisely for real lipid mixtures is problematic [76, 105] the determination of the phase diagram is possible. The phase diagrams (refer to Fig-

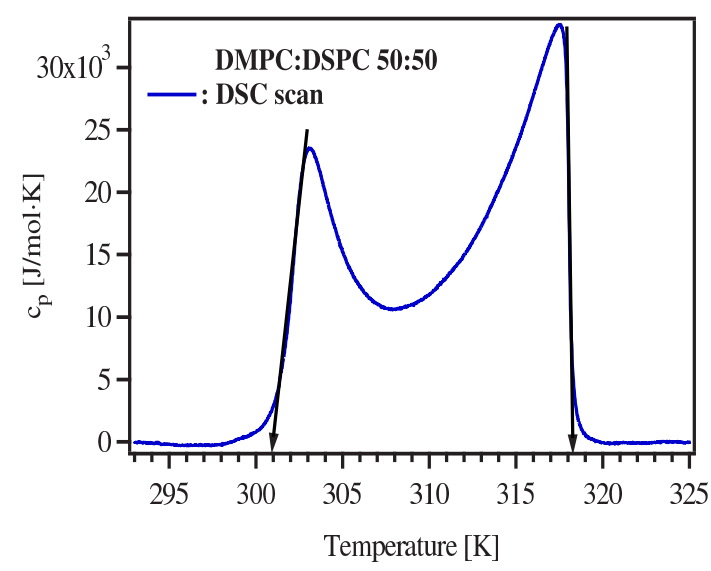

(a)

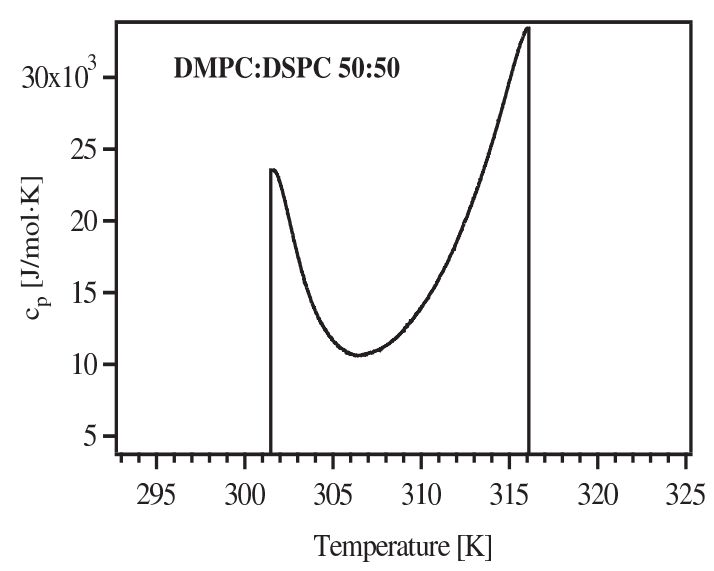

(b)

Figure 4.4: (a) The heat capacity curve of MLVs of 50:50 DMPC:DSPC mixture. Scan rate $5 K / h r$. (b) The heat capacity profile of a regular mixture. The transition begins exactly at the sharp temperature and also ends exactly at the sharp temperature.

ure 4.5 and Figure 4.6) show that the gel and fluid phases can coexist over a large temperature range. There are again regions in the gel phase (below the lower line), in the fluid phase (above the upper line) and between the two is a region in which the fluid and gel phase coexist (see Figure 4.5 and Figure 4.6). Phase diagram provides macroscopic phase separation.

With such a phase diagram, the number of lipids in a fluid and the number in a gel state can be estimated more precisely. For a 50:50 DMPC:DSPC mixture, the molar fraction of DSPC is taken as $Q=50$ at a given temperature. Then, the molar fraction of DSPC in gel phase is taken as $R$, and the molar fraction of DSPC in fluid phase is taken as $P$ (refer to Figure 4.6). Thus, the total fraction of both lipid species in a gel state is given by, 


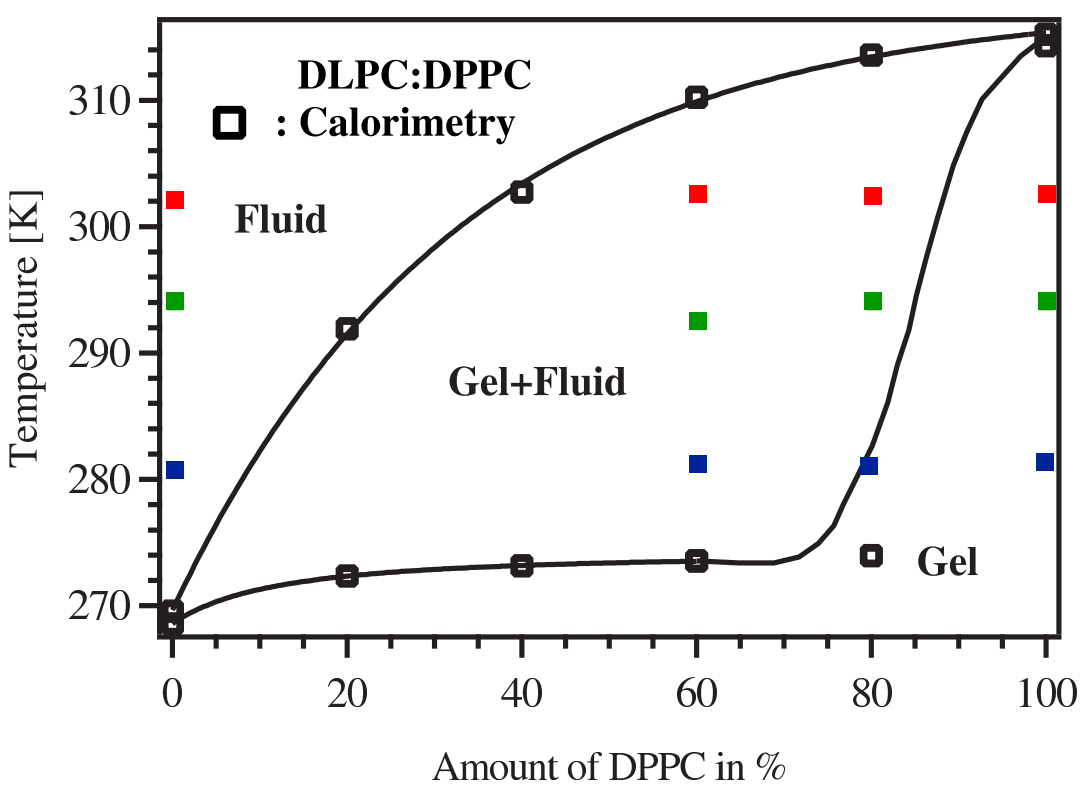

Figure 4.5: Phase diagram for a DLPC:DPPC mixture. Markers represent mixtures and temperatures at which FCS measurements were performed. The upper line is a limit between the fluid phase and the gel and fluid coexistence region, whereas the lower line between the gel phase and the gel and fluid coexistence region. The filled squares correspond to FCS experiments at a given temperature and for a given molar fraction of DPPC.

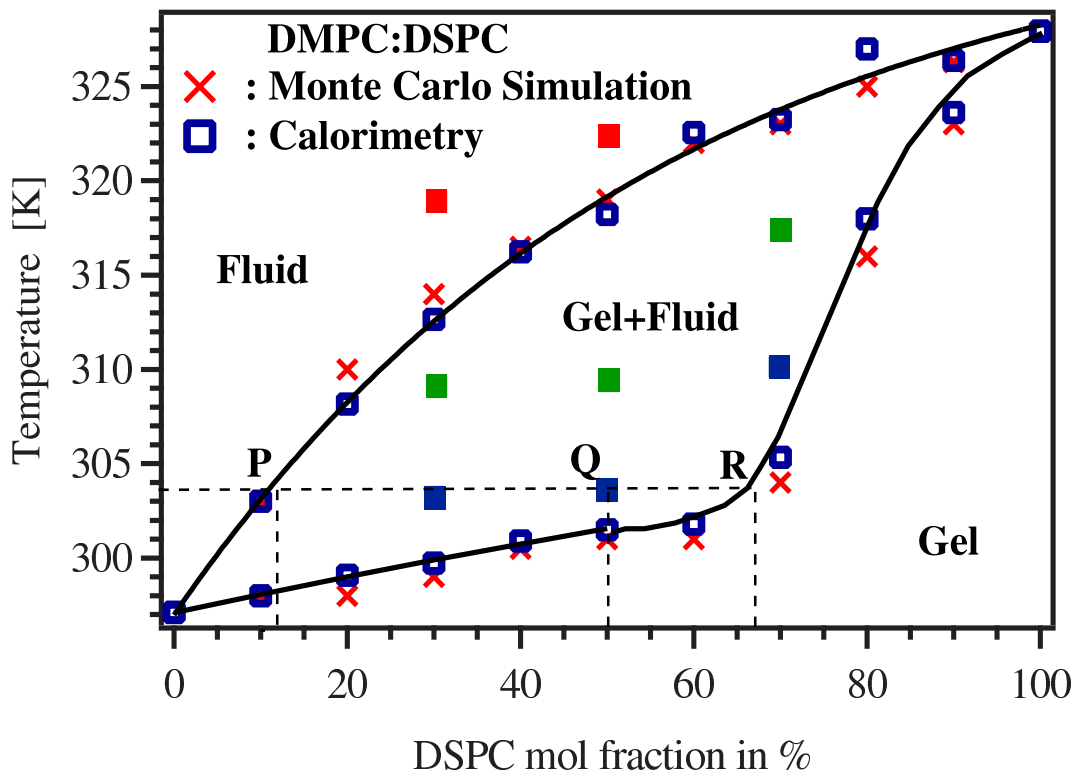

Figure 4.6: Phase diagram for a DMPC:DSPC mixture. The upper line is a limit between the fluid phase and the gel and fluid coexistence region, whereas the lower line between the gel phase and the gel and fluid coexistence region. The filled squares correspond to FCS experiments at a given temperature and for a given molar fraction of DSPC.

$$
f_{g}=\frac{Q-P}{R-P} .
$$


The total fraction of both lipid species in fluid state is given by,

$$
f_{l}=1-f_{g}=\frac{R-Q}{R-P}
$$

The expected $f_{\text {gel }}$ and measured $f_{\text {slow }}$ gel fractions and their standard deviations $\sigma_{f_{\text {slow }}}$ are shown in Table 4.1 for DLPC:DPPC lipid mixtures and in table 4.2 for DMPC:DSPC mixtures.

Table 4.1: The expected $\left(f_{\text {gel }}\right)$ and established $\left(f_{\text {slow }}\right)$ gel fractions from the FCS experiments (recorded in crosscorrelation mode) in DLPC:DPPC lipid mixtures with TRITC DHPE at various temperatures. $\sigma_{f_{s l o w}}$ is the standard deviation of The measured data with FCS slow fraction.

\begin{tabular}{|c|c|c|c|c|}
\hline $\begin{array}{c}\text { DLPC:DPPC } \\
\text { a molar fraction }\end{array}$ & $\begin{array}{c}\text { Temp. } \\
\mathrm{K}\end{array}$ & $\begin{array}{c}f_{\text {gel }} \\
\text { from DSC, } \%\end{array}$ & $\begin{array}{c}f_{\text {slow }} \\
\text { from FCS, \% }\end{array}$ & $\begin{array}{c}\sigma_{f_{\text {slow }}} \\
\text { from FCS, \% }\end{array}$ \\
\hline \multirow{3}{*}{$40: 60$} & 281.4 & 72 & 100 & - \\
& 292.8 & 61 & 44 & 13.4 \\
& 302.9 & 42 & 28.1 & 3.8 \\
\hline \multirow{3}{*}{$20: 80$} & 281.2 & 100 & 100 & - \\
& 294.3 & 90 & 62.8 & 8.13 \\
& 302.8 & 82 & 28.8 & 9.0 \\
\hline
\end{tabular}

The aim of the FCS experiments was to determine how many species are in the fluid phase (thus having a fast diffusion coefficient) and how many are in the gel phase (with a slow diffusion coefficient). For achieving this goal, a two component fitting procedure which assumes macroscopic phase separation (big gel and fluid domains) was used. Some deviations between the expected gel fractions from the phase diagram and those found in FCS experiments exist. In the FCS experiments the detected gel fraction depends on the partition of labels in the fluid and gel domains. Usually, dyes prefer a fluid phase as seen in the differential calorimetric measurements DSC (refer to Figure 3.13). In the presence of a label, the melting point is shifted to a lower temperature. This results in smaller values of detected gel fractions in FCS experiments, especially for DLPC:DPPC mixtures and for the DMPC:DSPC 30:70 mixture. The second explanation of lower detected gel fractions is photobleaching or photoquenching of slowly diffusing fluorescent dyes in gel domains. In the case of DMPC:DSPC mixtures, a good agreement between calorimetric and FCS measurements was obtained, whereas the even partition of the DiIC18 in gel and in fluid domains with DSC was observed. These values differ by maximally $18 \%$ of the expected gel fractions from DSC. Even for mixtures, DMPC:DSPC 50:50 and 70:30, higher values of the gel fractions in FCS experiments were obtained like in the calorimetric experiments. The gel fraction in pure DMPC and pure DSPC close to their melting temperatures were also measured. In 
Table 4.2: The expected $\left(f_{\text {gel }}\right)$ and established $\left(f_{\text {slow }}\right)$ gel fractions from FCS experiments (recorded in crosscorrelation mode) in DMPC:DSPC lipid mixtures with DiIC18 at various temperatures. $\sigma_{f_{s l o w}}$ is the standard deviation of the measured data with FCS slow fraction.

\begin{tabular}{|c|c|c|c|c|}
\hline $\begin{array}{c}\text { DMPC:DSPC } \\
\text { a molar fraction }\end{array}$ & $\begin{array}{c}\text { Temp. } \\
\mathrm{K}\end{array}$ & $\begin{array}{c}f_{\text {gel }} \\
\text { from DSC, } \%\end{array}$ & $\begin{array}{c}f_{\text {slow }} \\
\text { from FCS, \% }\end{array}$ & $\begin{array}{c}\sigma_{f_{\text {slow }}} \\
\text { from FCS, \% }\end{array}$ \\
\hline $100: 0$ & 296.6 & 50 & 76.5 & 9.1 \\
\hline \multirow{3}{*}{$30: 70$} & 291 & 100 & 100 & - \\
& 310.1 & 92 & 75.7 & 13.8 \\
& 317.6 & 68 & 57.5 & 11.9 \\
& 330 & 0 & 0 & - \\
\hline $50: 50$ & 290.5 & 100 & 100 & - \\
& 303.6 & 68 & 69.8 & 3.4 \\
& 309.7 & 51 & 65 & 2.2 \\
& 322.5 & 26 & 0 & - \\
\hline \multirow{3}{*}{$50: 30$} & 289.3 & 100 & 100 & - \\
& 303 & 40.4 & 44.9 & 20.5 \\
& 309.2 & 24 & 26.6 & 2.4 \\
\hline $0: 100$ & 319.2 & 0 & 0 & - \\
\hline & 328.2 & 50 & 56.5 & 22.5 \\
\hline
\end{tabular}

the case of DMPC, these values differ by $53 \%$ at its melting temperature. It is hypothesised that this may be due to the accuracy of the temperature measurement in a sample in the FCS experiments $( \pm 0.1 \mathrm{~K})$, since the melting transition has a very narrow half width of $\sim 0.05 \mathrm{~K}$ [45].

However, these deviations suggest that the fitting procedure, based on macroscopic phase separation may not be the adequate for estimating gel and fluid fractions. From the shape of correlation profiles in a coexistence region of gel and fluid phases, it is hypothesised that gel and fluid microstructures exist inside big domains. Microdomains have been visualised on Monte Carlo snapshots which include thermodynamical properties of lipid mixtures (Section 4.2.2. and 4.4.2). Thus, the fitting procedure requires revision based on this finding. 


\subsubsection{Monte Carlo snapshots}

With Monte Carlo (MC) simulations, heat capacity profiles over a broad temperature range can be simulated. On the basis of statistical thermodynamics a phase diagram can be constructed (see Section 2.2.2). From these simulations one can deduce the matrix snapshots. The MC snapshot is a picture of a matrix after the end of a Monte Carlo cycle. MC snapshots taken in the gel, in gel and fluid coexistence phases and in the pure fluid phase are presented in Figure 4.7. The dark symbols correspond to DSPC and the light symbols to DMPC.

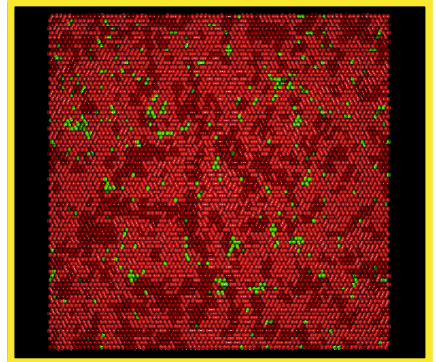

$289.3 \mathrm{~K}$

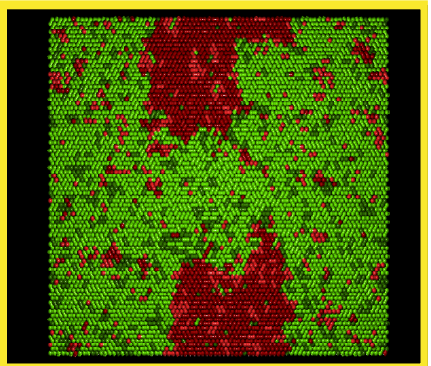

$308 \mathrm{~K}$

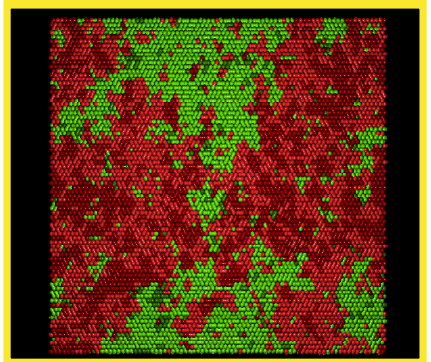

$301 \mathrm{~K}$

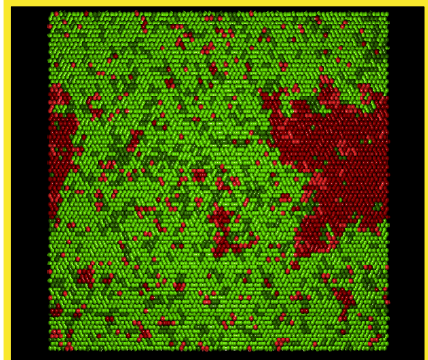

$310 \mathrm{~K}$

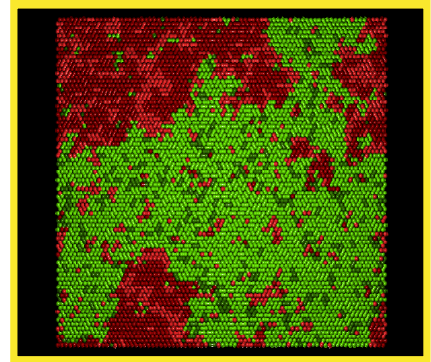

$305 \mathrm{~K}$

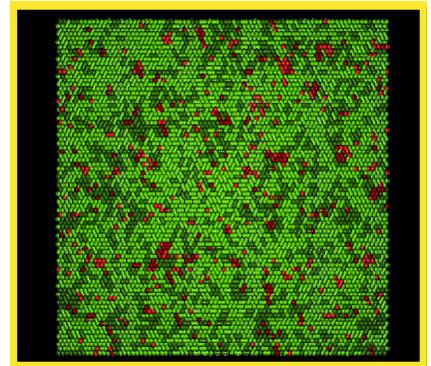

$319.2 \mathrm{~K}$

Figure 4.7: The Monte Carlo snapshots at 70 $\times 70$ of a DMPC:DSPC 70:30 mixture at different temperatures $\left(305 \mathrm{~K}, f_{g}=28 \% ; 308 \mathrm{~K}, f_{g}=19.6 \%\right)$. The first heat capacity maximum is at a temperature of $\mathrm{T}=301 \mathrm{~K}$ $\left(f_{g}=57 \%\right)$ and the second one at $T=310 K\left(f_{g}=13.2 \%\right)$. For the phase diagram, the temperatures $T=300 K$ and $T=313 \mathrm{~K}$ were determined according to the "tangent approximation".

The connection between points represents the chemical bond. The red symbols represent a gel phase and the green symbols a fluid phase. Gel and fluid macroscopic and microscopic domains are shown on the snapshots. At temperatures below the lower limit of the phase transition $(T=300 \mathrm{~K})$, almost all of lipids are in the gel state. There are only a few fluid micro-clusters. With increasing temperature, the amount of lipids in a fluid state increases as well as the size of fluid domains. In a gel and fluid coexistence region there is a macroscopic domain formation (i.e. huge gel and fluid domains). However, one can see the microscopic clusters of gel and fluid lipids at temperatures above $300 \mathrm{~K}$ and below $313 \mathrm{~K}$ [76; 105]. The number of fluid and gel chains increase with increasing matrix size in a way sim- 
ilar to the increasing in the mean size of the macroscopic clusters, whereas the mean size of microscopic clusters stays mainly constant. At higher temperatures, the number of gel-like lipids decreases and the size of gel macro-domains also decreases [110]. At temperatures above the upper limit of the phase transition $(T=313 \mathrm{~K})$, almost all of lipids are in a fluid phase whereas some gel microdomains exist.

\subsubsection{Confocal Fluorescence Microscopy}

The existence of domains, predicted through statistical thermodynamics, has been verified using Confocal Fluorescence Microscopy (CFM) and Atomic Force Microscopy (AFM) [42; 85]. With AFM, Mouritson has been able to detect domain formation directly in the transition region of DMPC and DSPC monolayers [85]. There are several papers of Bagatolli [6; 5; 22 that present domains in giant unilamellar vesicles (GUVs) of DLPC:DPPC, DMPC:DSPC and DMPE/DMPC mixtures at temperatures corresponding to the fluid-gel phase coexistence using CFM.

Fidorra and Schlötzer [99] of the Membrane Thermodynamics and Biophysics Group of MPI for biophysical Chemistry have observed domain formation in DLPC:DPPC, DMPC:DSPC mixtures using CFM. GUVs were prepared using the electro-formation method [6]. In that case, lipid-label solutions was spread between two ITO coverslips. The ITO coverslip was coated with Indium-Titanium-Oxide on one side in order to be electrically conductive as well as transmit light in the visible spectrum. Approximately, $2 \mu \mathrm{l}$ of $0.8 \mathrm{mg} / \mathrm{ml}$ concentration of the lipid-dye dissolved in $\mathrm{TFE}^{5}$ was spilled on the conducting side of one coverslip. The ratio, label to lipid, was kept on the level of 1 to 500. The dye absorbs light either at $488 \mathrm{~nm}$ or at $633 \mathrm{~nm}$. Lipids are labelled with two different dyes. The first dye prefers to partition mainly in a fluid phase and absorbs in a green wavelength while the second dye prefers to partition mainly in a gel phase and absorbs light at a red wavelength. The partitioning of labels into either gel or fluid domains was investigated using differential scanning calorimetry. The principle of these measurements is analogous to that described in a Section 3.2.1. A BODIPY dye, D-3803 ${ }^{6}$ with absorption maximum at $503 \mathrm{~nm}$ and emission maximum at $512 \mathrm{~nm}$ for a fluid phase and a DiD dye, D-77577 with absorption at $644 \mathrm{~nm}$ and emission at $663 \mathrm{~nm}$ for a gel phase in both mixtures were used. The solvent was evaporated under a stream of

\footnotetext{
${ }^{5} 2,2,2$ - Trifluoroethanol

6 2-(4,4-difluoro-5,7-dimethyl-4-bora-3a, 4a-diaza-s-indacene-3-pentanoyl)-1-hexadecanoyl-sn-glycero-3phosphocholine.

${ }^{7}$ 1,1'-dioctadecyl-3,3,3',3'- tetramethylindodicarbocyanine, 4-chlorobenzenesulfonate salt.
} 
nitrogen gas and then the ITO was left in a desiccator for more than 3 hours. Afterwards, a drop of water was added between the two coverslips, with their conducting sides facing each other, $1.5 \mathrm{~mm}$ apart. The water was heated to temperatures above melting transitions. Then an electrical AC-field as a sinusoidal wave function was applied with an amplitude of $3 \mathrm{~V}$ and frequency of $10 \mathrm{~Hz}$ for 10 to 30 minutes. In the presence of an electrical field, GUVs are formed. They grow from $5 \mu \mathrm{m}$ to $200 \mu \mathrm{m}$ in a diameter. The mean size of GUVs was approximately $30 \mu \mathrm{m}$. A scan rate of $200 \mu \mathrm{s}$ was used to acquire an image every $1 \mu \mathrm{m}$ for both dyes. The superposition of the GUV images, taken with the red laser and with the green laser, was performed. Domains where gel and fluid phases coexist are shown in Figure 4.8 for the DLPC:DPPC mixture, and in Figure 4.9 for the DMPC:DSPC mixture [unpublished results].

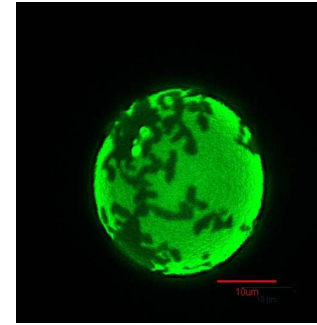

(a)

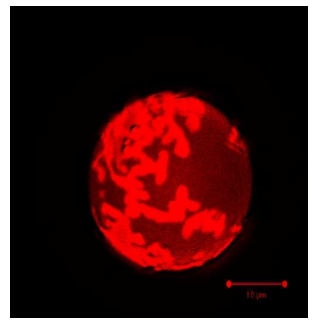

(b)

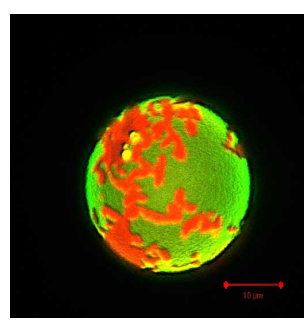

(c)

Figure 4.8: An example of domain formation in the DLPC:DPPC 30:70 mixture at room temperature. (a) The green fluorescence of BODIPY dye partitioning in a fluid phase. (b) The red fluorescence of DiD dye partitioning in a gel phase. (c) The superposition of fluid and gel domains. Bars correspond to $10 \mu \mathrm{m}$. (Courtesy Fidorra from Membrane Thermodynamics and Biophysics Group of MPI for biophysical Chemistry.)

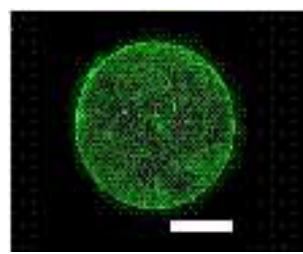

(a)

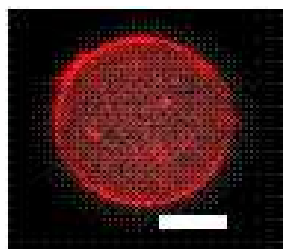

(b)

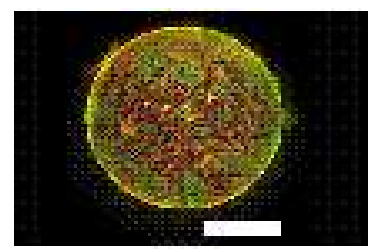

(c)

Figure 4.9: An example of domain formation in the DMPC:DSPC 30:70 mixture at $298 \mathrm{~K}$. (a). The green fluorescence of BODIPY dye partitioning in a fluid phase. (b) The red fluorescence of DiD dye partitioning in a gel phase. (c) The superposition of fluid and gel domains. Bars correspond to $10 \mu \mathrm{m}$. (Courtesy Fidorra from Membrane Thermodynamics and Biophysics Group of MPI for biophysical Chemistry.)

Different shapes of domains in the gel-fluid phase coexistence region were observed. The domain shape depends on the phospholipid composition and on the temperature. In the case 
of the DLPC:DPPC 30:70 mixture, the gel domains are presented as many of thin but long domains connected with each other and surrounding vesicles. In the case of the DMPC:DSPC mixtures, the gel domains show a "dendritic shape". Recent studies observed that with decreasing temperature gel domains expand and migrate around vesicles [36; 92].

Giant unilamellar vesicles are large, thereby they have a small radius of curvature and their surface can be treated as a flat area. These "cell size" vesicles are objects of interest for good cell membranes model systems and for investigating membrane behaviour [6; 22]. The advantage of GUVs is that they represent a free unilamellar membrane without wall contact. However, there are undulations on the surface which can be interpreted as diffusion [5]. For this reason, multilamellar membranes supported on a coverslip were used. Another reason to reject GUVs for diffusion measurements was that their generation was not reproducible.

\subsection{Diffusion in a DLPC:DPPC mixture}

It was obvious from calorimetric measurements and from Confocal Fluorescence Microscopy (CFM) that there is a phase coexistence region which can influence diffusion processes in membranes. Diffusion processes and domain formation indirectly by Fluorescence Correlation Spectroscopy (FCS) were investigated.

Firstly the diffusion processes in a DLPC:DPPC mixture was studied since it has gel and fluid coexistence phase already at room temperature ( $\approx 298 \mathrm{~K})$. Cooperation with the Biophysics group of MPI for biophysical Chemistry led by Prof. Schwille was sought for the investigation of diffusion in GUVs. The values of diffusion coefficients coming from both experiments are in a reasonable agreement [58].

Multilamellar DLPC:DPPC membranes on a quartz support by FCS were investigated. The used phospholipids differ in chain length by four methylene groups. The fluorescent signal was detected by two avalanche photodiodes (APD) and correlated on-line in crosscorrelation or autocorrelation mode. Lipids were labelled with a fluorescence marker: TRITC-DHPE and hydrated with bidistilled water saturated with oxygen. Using the crosscorrelation mode and oxygen gas, one reduces noise on short time scales $(\mu \mathrm{s})$, whereas crosscorrelation mode eliminates the electronical noise of photodiodes and the oxygen quenches the triplet state (see Section 3.1.2.). Some experiments with a $30 \mu \mathrm{m}$ pinhole were performed to show that the diffusion coefficient does not depend on the focus size (see Figure 4.10). In that case, time scales were calibrated with rhodamine $6 \mathrm{G}$ and were measured with a $30 \mu \mathrm{m}$ pinhole. 
The presented correlation curves were normalised by the multiplication with a mean number of molecules $\langle N\rangle$ and the subtraction of the value of a base line $B$ which varies around 1. Measurements for pure DLPC, DLPC:DPPC 40:60, DLPC:DPPC 20:80 and pure DPPC,

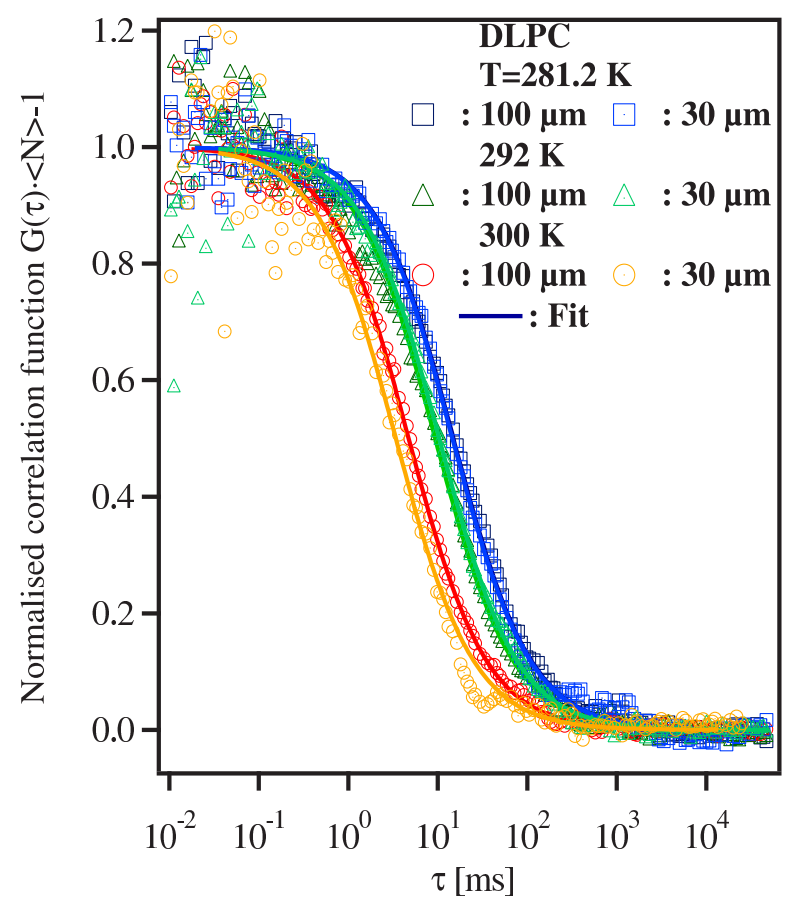

Figure 4.10: Normalised crosscorrelation profiles of TRITC DHPE diffusing in a DLPC membranes done with $100 \mu \mathrm{m}$ (dark red, green, blue) and $30 \mu \mathrm{m}$ (light red, gree, blue colours) pinhole. Markers are from FCS measurements, solid lines represent one component fits.

each at three-four different temperatures corresponding to the fluid phase, the gel and fluid coexistence phase and the gel phase respectively were also performed. The amount of a gel phase was regulated by changing the molar fraction of DPPC and by changing temperature. At every temperature, several samples at several different regions were measured, resulting in approximately 20 measurements with a duration of $120 \mathrm{~s}$ (fluid phase) or $300 \mathrm{~s}$ (gel phase). The fluorescent signal from the gel phase was recorded for longer times in order to have a good quality of correlation curve, since label diffusion occurs slower. Count rates were typically between 5 and $200 \mathrm{kHz}$ and the background noise was less than $2 \mathrm{kHz}$. The count rate per molecule was 1.2 to $2 \mathrm{kHz}$.

In a pure fluid and in a pure gel phase, it was hypothesised that lipids are in one of the phases: either in a gel or in a fluid phase. Therefore, pure fluid and pure gel phases were fitted with a one component diffusion coefficient fit using the following equation, 


$$
G(\tau)=B+\frac{1}{\langle N\rangle} \cdot\left(\frac{1}{1+\frac{\tau}{\tau_{D}}}\right),
$$

where: $B$ is the value of a base line which was around 1 ,

$\langle N\rangle$ is the mean number of dye in a focus and

$\tau_{D}$ is the diffusing time of label through the focus.

The diffusion coefficient, in both cases, was calculated using Eq. 2.5 and the standard deviation according to the equation,

$$
\sigma_{\left\langle D_{\tau}\right\rangle}=\sqrt{\frac{2 r_{0} \cdot \sigma_{r_{0}}}{\left(4 \tau_{D}\right)^{2}}+\frac{r_{0}^{2} \cdot \sigma_{\tau_{D}}}{\left(4 \tau_{D}\right)^{2}}},
$$

where: $r_{0}, \sigma_{r_{0}}$ are the focus radius and its standard deviation and

$\sigma_{\tau_{0}}$ is the standard deviation of the diffusing time.

The representative normalised correlation profiles for DLPC in a fluid phase and for DPPC in a gel phase are graphically depicted in Figure 4.11 and Figure 4.12.

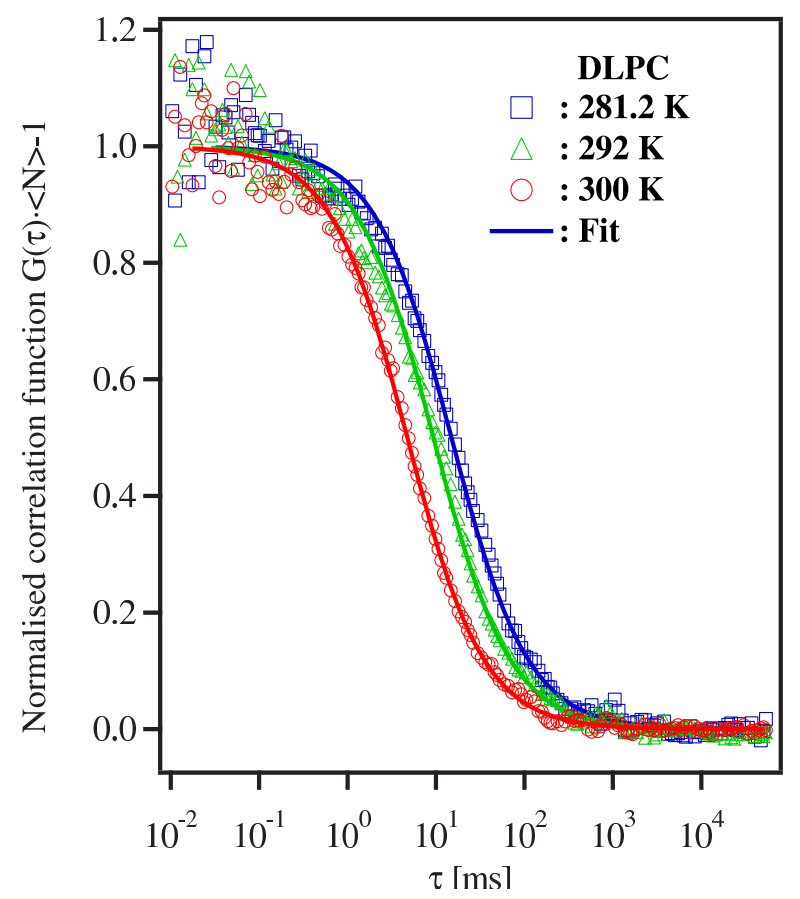

Figure 4.11: Normalised cross-correlation profiles of TRITC DHPE diffusing in DLPC membranes at various temperatures. Markers represent FCS measurements, solid lines represent one component fits. 


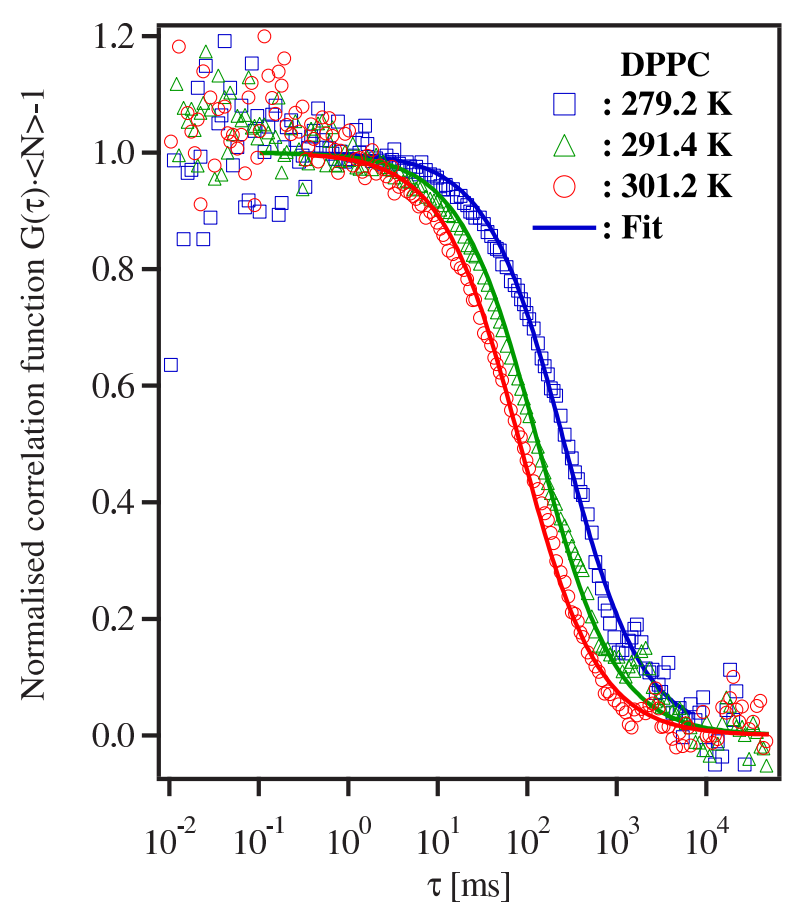

Figure 4.12: Normalised cross-correlation profiles of TRITC DHPE diffusing in DPPC membranes at various temperatures. Markers represent FCS measurements, solid lines represent one component fits.

For mixtures with gel and fluid coexistence phases, a simple diffusion model was not sufficient to describe the correlation profiles. Therefore, a two component diffusion coefficient fit, assuming superposition of slow and fast diffusing species in a gel and a fluid domain respectively, was used. The data were fitted according to,

$$
G(\tau)=B+\frac{1}{\langle N\rangle} \cdot\left(\frac{f_{\text {slow }}}{1+\frac{\tau}{\tau_{D_{\text {slow }}}}}\right)+\left(\frac{1-f_{\text {slow }}}{1+\frac{\tau}{\tau_{D_{\text {fast }}}}}\right),
$$

where: $f_{\text {slow }}, 1-f_{\text {slow }}$ is the expected fraction with either a slow or a fast diffusion constant respectively and,

$\tau_{D_{\text {slow }}}, \tau_{D_{\text {fast }}} 8$ is the diffusing time of label through the focus of a slow or a fast component respectively.

With the two component fit, two macroscopic phases are assumed: big gel and big fluid ones (but no microstructure).

In Figure 4.13 and Figure 4.14 , the representative normalised curves for every mixture at

\footnotetext{
${ }^{8}$ We called them a slow and a fast diffusing component since there is not macroscopic phase separation ( two big pure gel and pure fluid phases) but there are micro-heterogenities, see also discussion in chapter 4.2.2. and 4.4
} 
each temperature are shown.

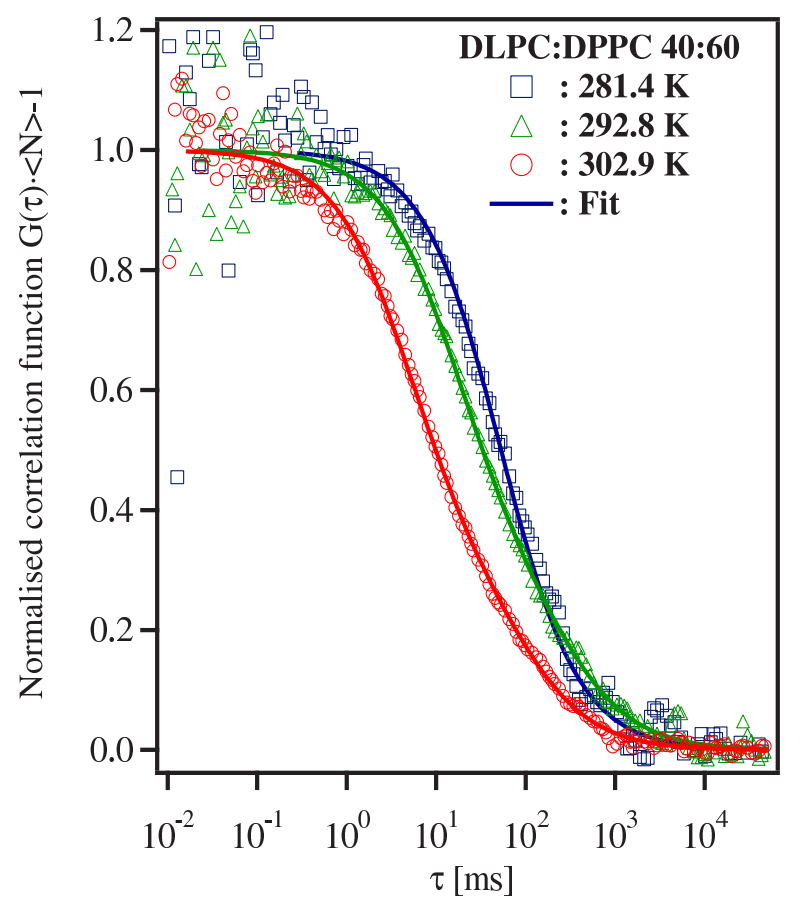

Figure 4.13: Normalised cross-correlation profiles of TRITC DHPE diffusing in a DLPC:DPPC 40:60 mixture at various temperatures. Markers represent FCS measurements, solid lines represent one component fits at $281.4 \mathrm{~K}$ and at $302.9 \mathrm{~K}$, or a two component fit at $292.8 \mathrm{~K}$.

The average values of translational diffusion coefficients, $\left\langle D_{\tau}\right\rangle=\frac{D_{\tau}}{n}$, over $n$ measurements and their standard deviations $\sigma_{\left\langle D_{\tau}\right\rangle}$, are shown in Table 4.5 and in Table 4.4. The relative standard deviation varies with the sample preparation and makes up a maximum of $27 \%$ of the measured diffusion coefficient what is a good result for measurements on soft material samples [77].

The FCS curves from each measurement were repeatable to within $25 \%$. In the cases of gel and fluid phase coexistence, they were more scattered that sometimes a one component or both components at one temperature overlap FCS curves at slightly different temperatures. However, the change for different conditions can clearly be seen (see Figure 4.15).

The measurements performed in crosscorrelation mode give the same results within the standard deviation, similar to the measurements in autocorrelation mode, see Figure . 4.15.

In both modes a fast diffusion coefficient in fluid domains at high temperature, e.g. DLPC at $302 \mathrm{~K}: D_{\tau}=6.72 \cdot 10^{-8} \mathrm{~cm}^{2} / \mathrm{s}$ was observed in FCS experiments in comparison to those in gel domains at low temperature e.g. DPPC, $281.7 \mathrm{~K}: D_{\tau}=2.08 \cdot 10^{-9} \mathrm{~cm}^{2} / \mathrm{s}$.

Different diffusion behaviour in the gel and fluid coexistence region (like in pure fluid and 


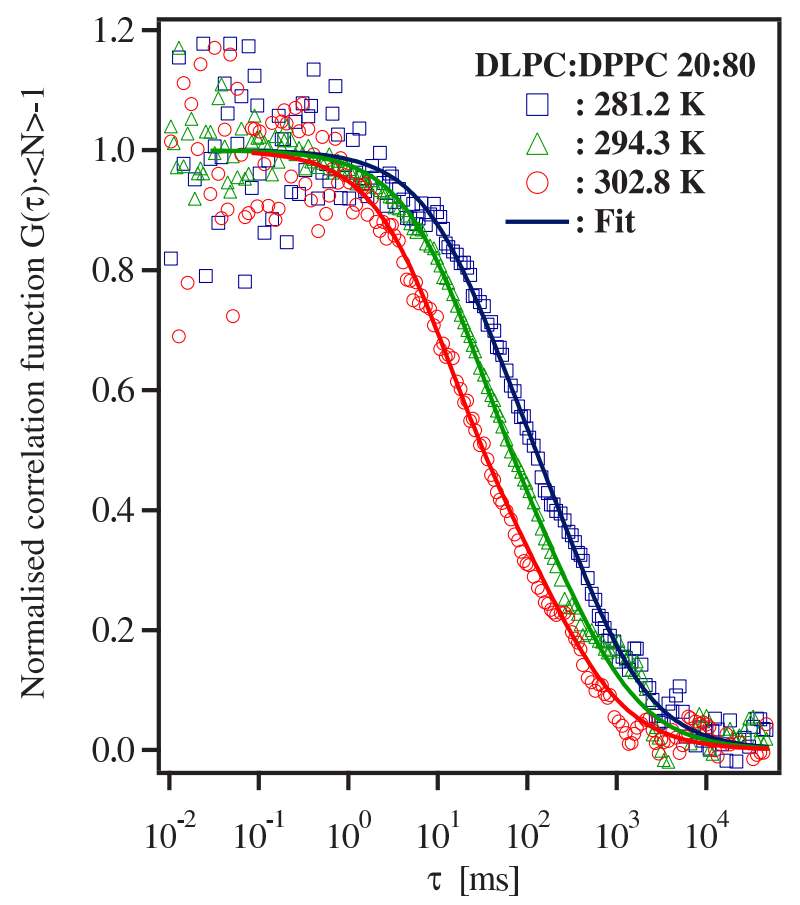

Figure 4.14: Normalised cross-correlation profiles of TRITC DHPE diffusing in a DLPC:DPPC 20:80 mixture at various temperatures. Markers represent FCS measurements, solid lines represent one component fits at $281.2 \mathrm{~K}$ and $302.8 \mathrm{~K}$, or a two component fit at $294.3 \mathrm{~K}$.

Table 4.4: Average translational diffusion coefficients of a slow component, $\left\langle D_{\tau_{\text {slow }}}\right\rangle$, and of a fast component, $\left\langle D_{\tau_{f a s t}}\right\rangle$, in DLPC:DPPC lipid mixtures at various temperature measured by FCS. $\sigma_{\left\langle D_{\tau}\right\rangle}$ is the standard deviation of an average $\left\langle D_{\tau}\right\rangle$. Experiments were recorded in crosscorrelation mode. (-) means that at a given temperature, a gel or a fluid phase does not exist.

\begin{tabular}{|c|c|c|c|c|c|}
\hline $\begin{array}{c}\text { DLPC:DPPC } \\
\text { a molar fraction }\end{array}$ & $\begin{array}{c}\text { Temp. } \\
\text { K }\end{array}$ & $\begin{array}{c}\left\langle D_{\tau_{\text {fast }}}\right\rangle \\
\cdot 10^{-8 \frac{\mathrm{cm}^{2}}{\mathrm{~s}}}\end{array}$ & $\begin{array}{c}\sigma_{\left\langle D_{\tau_{\text {fast }}}\right\rangle} \\
\cdot 10^{-8} \frac{\mathrm{cm}^{2}}{\mathrm{~s}}\end{array}$ & $\begin{array}{c}\left\langle D_{\tau_{\text {slow }}}\right\rangle \\
\cdot 10^{-9} \frac{\mathrm{cm}^{2}}{\mathrm{~s}}\end{array}$ & $\begin{array}{c}\sigma\left\langle D_{\tau_{\text {slow }}}\right\rangle \\
\cdot 10^{-9} \frac{\mathrm{cm}^{2}}{\mathrm{~s}}\end{array}$ \\
\hline \multirow[t]{3}{*}{$100: 0$} & 281.2 & $2.27^{s}$ & $0.62^{s}$ & - & - \\
\hline & 294.0 & 3.21 & 0.46 & - & - \\
\hline & 302.0 & 6.72 & 0.12 & - & - \\
\hline \multirow{3}{*}{$40: 60$} & 281.4 & - & - & 8.09 & 0.15 \\
\hline & 292.8 & 2.48 & 0.68 & 2.48 & 0.11 \\
\hline & 302.9 & 6.68 & 0.81 & 3.64 & 0.71 \\
\hline \multirow{3}{*}{$20: 80$} & 281.2 & - & - & 2.25 & 0.17 \\
\hline & 294.3 & 3.79 & 0.12 & 2.31 & 0.14 \\
\hline & 302.8 & 4.23 & 0.19 & 2.69 & 0.66 \\
\hline \multirow{3}{*}{$0: 100$} & 281.7 & - & - & 2.08 & 0.55 \\
\hline & 293.4 & - & - & 1.83 & 0.15 \\
\hline & 302.8 & - & - & 2.53 & 0.13 \\
\hline
\end{tabular}


Table 4.5: Average translational diffusion coefficients of a slow component, $\left\langle D_{\tau_{s l o w}}\right\rangle$, and of a fast component, $\left\langle D_{\tau_{\text {fast }}}\right\rangle$, in DLPC:DPPC lipid mixtures at various temperature measured by FCS. $\sigma_{\left\langle D_{\tau}\right\rangle}$ is the standard deviation of an average $\left\langle D_{\tau}\right\rangle$. Experiments were recorded in autocorrelation mode. (-) means that at a given temperature a gel or a fluid phase does not exist.

\begin{tabular}{|c|c|c|c|c|c|}
\hline $\begin{array}{c}\text { DLPC:DPPC } \\
\text { a molar fraction }\end{array}$ & $\begin{array}{c}\text { Temp. } \\
\text { K }\end{array}$ & $\begin{array}{c}\left\langle D_{\tau_{\text {fast }}}\right\rangle \\
\cdot 10^{-8} \frac{\mathrm{cm}^{2}}{\mathrm{~s}}\end{array}$ & $\begin{array}{l}\left.\sigma_{\left\langle D_{\tau_{\text {fast }}}\right\rangle}\right\rangle \\
\cdot 10^{-8} \frac{\mathrm{cm}^{2}}{s}\end{array}$ & $\begin{array}{c}\left\langle D_{\tau_{\text {slow }}}\right\rangle \\
\cdot 10^{-9} \frac{\mathrm{cm}^{2}}{\mathrm{~s}}\end{array}$ & $\begin{array}{l}\sigma\left\langle D_{\tau_{\text {slow }}}\right\rangle \\
\cdot 10^{-9} \frac{\mathrm{cm}^{2}}{\mathrm{~s}}\end{array}$ \\
\hline \multirow{3}{*}{ 100:0 } & 281.2 & $2.37^{\circ}$ & 0.52 & - & - \\
\hline & 294.0 & 3.22 & 0.54 & - & - \\
\hline & 302.0 & 7.79 & 0.81 & - & - \\
\hline \multirow{3}{*}{$40: 60$} & 281.4 & - & - & 7.62 & 0.53 \\
\hline & 292.8 & 2.86 & 0.54 & 2.29 & 0.11 \\
\hline & 302.9 & 4.62 & 0.11 & 3.01 & 0.17 \\
\hline \multirow{3}{*}{$20: 80$} & 281.2 & $\begin{array}{ll}- & -1\end{array}$ & - & 3.52 & 0.29 \\
\hline & 294.3 & 3.19 & 0.86 & 2.28 & 0.14 \\
\hline & 302.8 & 4.14 & 0.24 & 3.03 & 0.29 \\
\hline \multirow{3}{*}{$0: 100$} & 281.7 & - & - & 2.22 & 0.35 \\
\hline & 293.4 & - & - & 1.94 & 0.31 \\
\hline & 302.8 & - & - & 2.31 & 0.11 \\
\hline
\end{tabular}

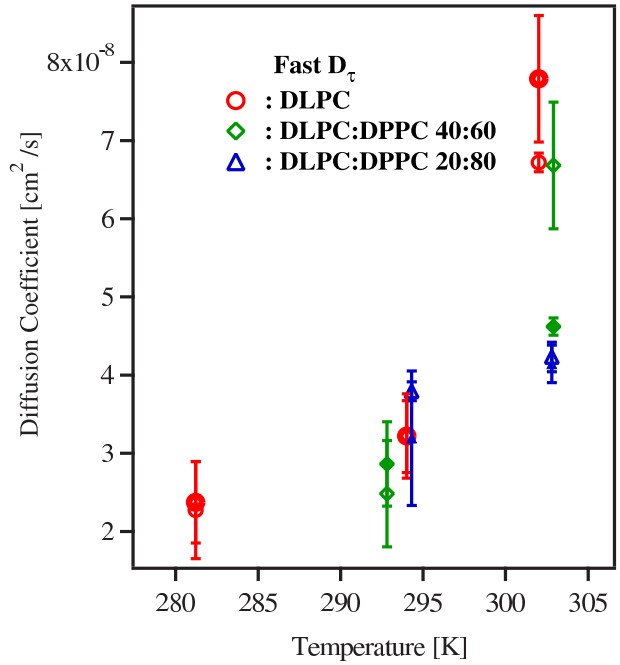

(a)

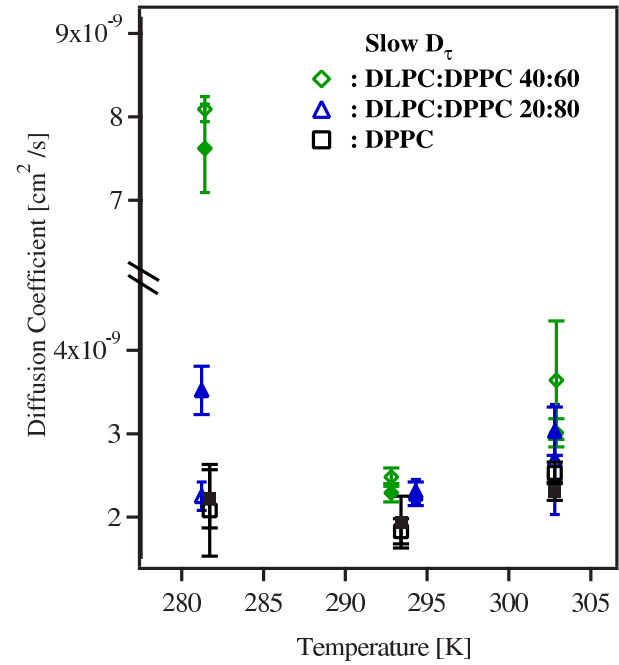

(b)

Figure 4.15: Diffusion coefficient of TRITC DHPE in various DLPC:DPPC mixtures of a fast component (a) and a slow component (b) as a function of temperature. The open symbols represent measurements performed in the croscorrelation mode, whereas the filled symbols in the autocorrelation mode.

pure gel phases) were noted. Lipid mixtures at temperatures corresponding to the coexistence phases were fitted assuming superposition of two diffusion coefficients, as a result of the gel 
and fluid domain coexistence.

The diffusion coefficient of the slow component, (e.g. $D_{\tau}=2.48 \cdot 10^{-8} \mathrm{~cm}^{2} / \mathrm{s}$ for DLPC:DPPC 40:60 at $\left.292.8 \mathrm{~K}, f_{g}=61 \%\right)$ is faster than $D_{\tau}$ in a pure gel phase $\left(\right.$ e.g. $D_{\tau}=2.48 \cdot 10^{-9} \mathrm{~cm}^{2} / \mathrm{s}$ for DPPC at $293.4 \mathrm{~K}$ ) at a corresponding temperature (see Table 4.4 and 4.5 ). The diffusion of a fast component (e.g. $4.23 \cdot 10^{-8} \mathrm{~cm}^{2} / s$ for DLPC:DPPC 20:80 at $302.8 \mathrm{~K}$, $f_{l}=82 \%$ ) is slower than the diffusion in a pure fluid phase e.g. $6.72 \cdot 10^{-8} \mathrm{~cm}^{2} / \mathrm{s}$ for DLPC at $302 \mathrm{~K}$ at the same temperature (see Figure 4.13, Figure 4.14).

This may indicate that there are gel microdomains inside huge fluid domains, and fluid microdomains inside big gel domains.

Microdomains have been seen on Monte Carlo snapshots of DMPC:DSPC mixtures therefore such a membrane picture would be now introduced. In Figure 4.16 is showed an example of a MC snapshoot where micro- and macrodomians occur is presented. The gel microdomains

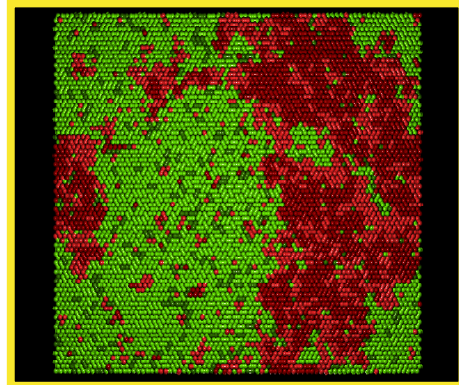

$303 \mathrm{~K}$

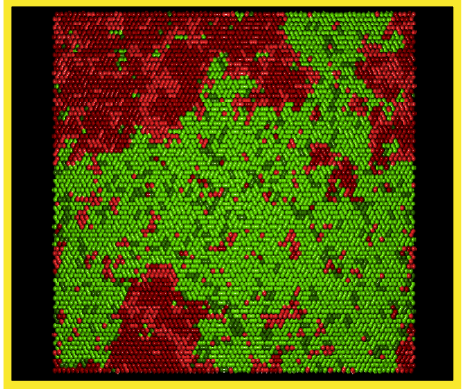

$305 \mathrm{~K}$

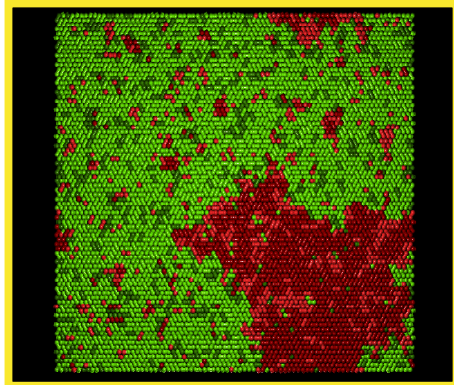

$307 \mathrm{~K}$

Figure 4.16: The Monte Carlo snapshots $70 \times 70$ of DMPC:DSPC 70:30 mixture at temperatures corresponding to the gel and fluid coexistence phase $\left(303 \mathrm{~K}, f_{g}=40.4 \% ; 305 \mathrm{~K}, f_{g}=28 \% ; 307 \mathrm{~K}, f_{g}=26 \%\right)$. The dark symbols correspond to DSPC and the light symbols to DMPC. The red symbols represent lipids in a gel phase and the green symbols in a fluid phase. The connection between points represents the chemical bond.

contain only a few DMPC and DSPC lipids in a gel state. The fluid microdomains contain only few lipids with a shorter and with a longer chain length in a fluid state. The presence of such microdomains requires revision of the two component fitting procedure for coexistence phases. The best example for failure using this fitting procedure is the mixture of DLPC:DPPC 40:60 at 281.4 K (refer to Tables 4.4 and 4.5). From the phase diagram, approximately $30 \%$ of the lipids should be in a fluid phase (refer to Table 4.1). It was not possible to mark off the fluid and gel parts with two component fits. However, the mean diffusion coefficient, equal to $8.09 \cdot 10^{-9} \mathrm{~cm}^{2} / \mathrm{s}$, is almost four times faster than the $D_{\tau}$ in a gel phase at the corresponding temperature $2.08 \cdot 10^{-9} \mathrm{~cm}^{2} / \mathrm{s}$. It is almost three times slower than $D_{\tau}$ in a fluid phase at the same temperature $\left(2.27 \cdot 10^{-8} \mathrm{~cm}^{2} / \mathrm{s}\right)$.

This may indicate that there is no macroscopic phase separation. On the other hand, it may predict that there are microscopic fluid and gel domains because the diffusion coefficient has 
values between $D_{\tau}$ in a fluid phase and $D_{\tau}$ in a gel phase. The size of these domains may be smaller than the diameter of the focus $(754 \mathrm{~nm})$, thus they cannot be separated by the fitting procedure.

Another source of deviations is the curves fitting procedure which assumes a Gaussian shape for the focus. Hess and Webb found that if the focal volume has a different shape, then it occurs in the FCS curve as an additional exponential component, as discussed in Section 2.2.1 [46; 47]. Both assumptions (macroscopic phase separation and the Gaussian profile of the focus) results in small misinterpretations of the correlation curves.

Obviously, the shape of the correlation profile reflects the coexistence of gel and fluid domains. A decrease in temperature results in a lower mean diffusion coefficient caused by the relative increase in the gel domain area with respect to the fluid domains (see Figure 4.17). To understand better diffusion processes, microdomain and domain formation in lipid mem-

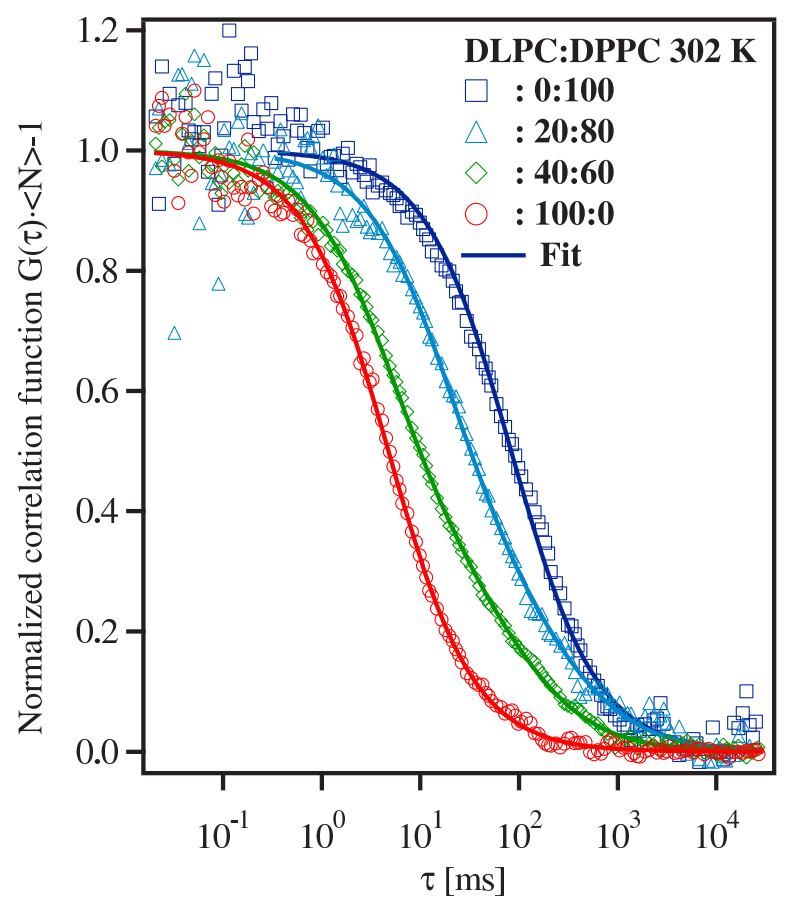

Figure 4.17: Normalised cross-correlation profiles of TRITC DHPE diffusing in DLPC, DLPC:DPPC 40:60, DLPC:DPPC 20:80 and DPPC stocks of membranes at $302 \mathrm{~K}$. Symbols are from FCS measurements, solid lines represent one component fits for DLPC and DSPC, and two component fits for mixtures.

branes, it was decided to perform Monte Carlo simulations on the basis of the thermodynamical properties of the lipid system. 


\subsection{Diffusion in a DMPC:DSPC mixture}

Since a control of the temperature during measurements over a wide temperature range $(253 \mathrm{~K}$ to $353 \mathrm{~K})$ is possible the diffusion processes in different mixtures that have the gel and fluid coexistence phase at temperatures other than room temperature can be studied. Therefore, DMPC:DSPC mixtures were investigated. The main reason for choosing a DMPC:DSPC membrane is the large knowledge about this system. Already, Sugar has analysed this system with a Monte Carlo simulation similar to the model currently employed [110; 111 .

\subsubsection{FCS results}

The diffusion in artificial hydrated multilamellar membranes supported on a coverslip were investigated. Mixtures of different DMPC and DSPC molar fractions labelled with the fluorescence marker DiIC18 were used. These phospholipids differ in length by four carbon atoms. The temperature of the experiment was regulated to vary the amount of a gel phase in every DMPC:DSPC mixture. Approximately 20 measurements were carried out over times of $120 \mathrm{~s}$ (fluid phase) or $300 \mathrm{~s}$ (gel phase). For each sample and each temperature, a series of measurements was made. The area under investigation was also changed. Count rates were between 2 and $50 \mathrm{kHz}$, the background noise was less than $2 \mathrm{kHz}$. The count rate per molecule was equal to $1 \mathrm{kHz}$ in all measurements.

Normalised ${ }^{9}$ crosscorrelation profiles of DiIC18 diffusing in planar DMPC and DSPC bilayers are graphically depicted (see Figure 4.18, and Figure 4.19). The measured correlation curves were fitted according to a one-component diffusion coefficient fit for a pure gel and a pure fluid phase (refer to Eq.4.3) and with a two-component diffusion coefficient fit for a gel and fluid coexistence phase (refer to Eq. 4.5).

The average values of the translational diffusion coefficient, $\left\langle D_{\tau}\right\rangle=\frac{D_{\tau}}{n}$, over $n$ measurements and their standard deviations, $\sigma_{\left\langle D_{\tau}\right\rangle}$ are shown in Table 4.6 and Table 4.7. The relative standard deviation in determination of $\left\langle D_{\tau}\right\rangle$ is a maximum of $40 \%$ of the measured diffusion coefficient.

For both autocorrelation and crosscorrelation modes, good agreement within standard deviation of the diffusion coefficient values was obtained (compare Table 4.6 with Table 4.7 ,

\footnotetext{
${ }^{9}$ Normalisation means the multiplication with a mean number of molecules $\langle N\rangle$ and the subtraction of the value of a base line $B$ which varies around 1 .
} 


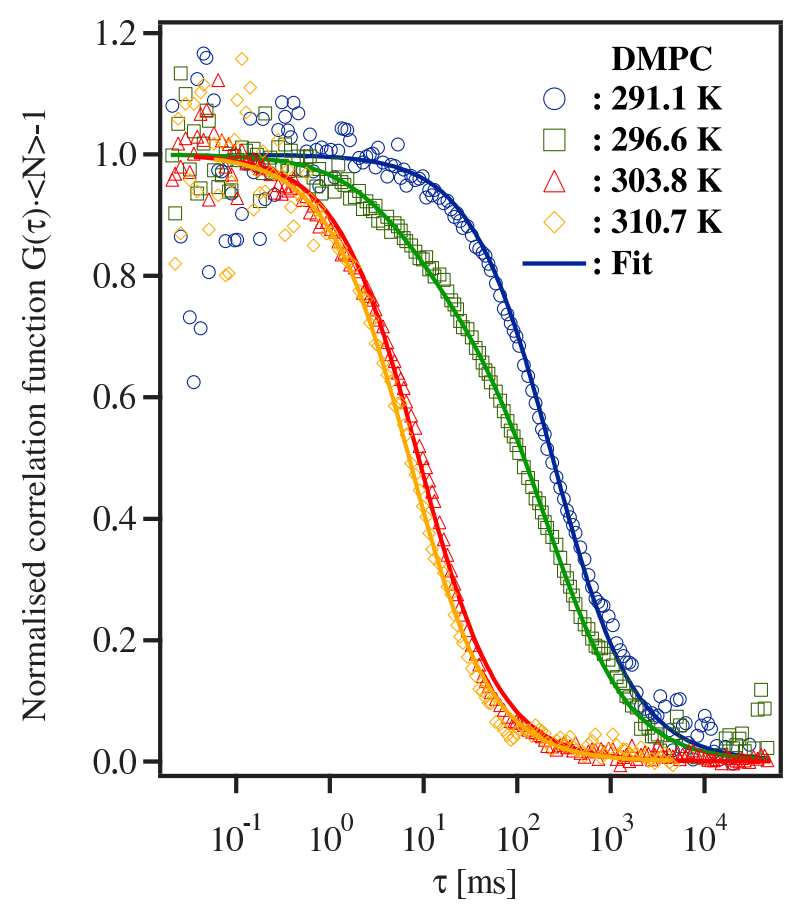

Figure 4.18: Normalised cross-correlation profiles of DiIC18 diffusing in DMPC membranes at various temperatures. Markers represent FCS measurements, solid lines represent a two component fit at $296.6 \mathrm{~K}$ whereas at the other temperatures represents one component fits.

see Figure 4.20). Since this was seen it in the DLPC:DPPC mixtures, it was decided to perform measurements only in crosscorrelation mode.

The FCS profiles from each measurement were highly reproducible (see Figure 4.21). It was difficult to make a detailed error analysis, because the standard deviation in measured FCS curves depended also on how the probes are prepared (e.g. hydration rate) [77]. Figure 4.21 gives an impression of the typical scatter of the FCS profiles in a gel phase, a gel and fluid coexistence phase and a fluid phase. Although the FCS profiles are spread out, it can been seen that experiments performed on the different conditions (temperatures) are clearly separate from each other and the trends are visualised easily. The middle curve at every temperature has been chosen as the most representative curve (red curves) for comparison with MC simulations (see Figure 4.21).

In a pure gel, the diffusion coefficient, $D_{\tau}$, is equal to $0.86 \cdot 10^{-9} \mathrm{~cm}^{2} / \mathrm{s}$ (DSPC at $290.6 \mathrm{~K}$ ), whereas in a pure fluid it is equal to $5.04 \cdot 10^{-8} \mathrm{~cm}^{2} / \mathrm{s}$ (DSPC at $330.4 \mathrm{~K}$,).

For DMPC membranes in a pure gel, $D_{\tau}$ is equal to $1.48 \cdot 10^{-9} \mathrm{~cm}^{2} / \mathrm{s}$ (DMPC at $291.1 \mathrm{~K}$ ), whereas in a pure fluid it is equal to $4.84 \cdot 10^{-8} \mathrm{~cm}^{2} / \mathrm{s}$ (DMPC at $310.7 \mathrm{~K}$ ). 


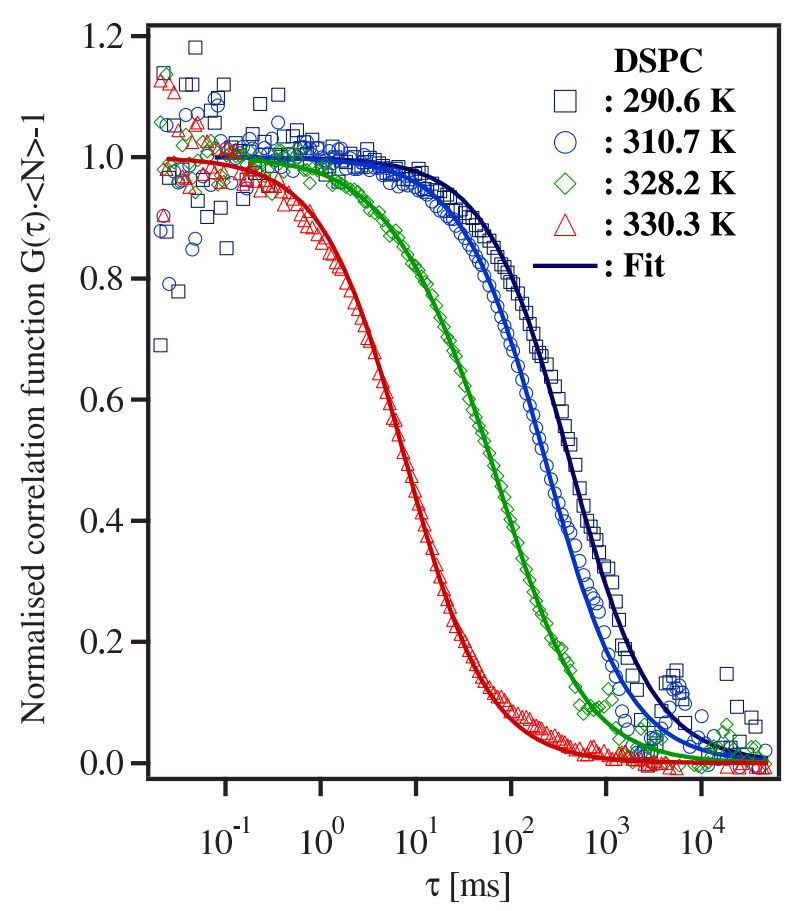

Figure 4.19: Normalised crosscorrelation profiles of DiIC18 diffusing in DSPC membranes at various temperatures. Markers represent FCS measurements, solid lines represent a two component fit at $328.2 \mathrm{~K}$ whereas at the other temperatures represent one component fits.

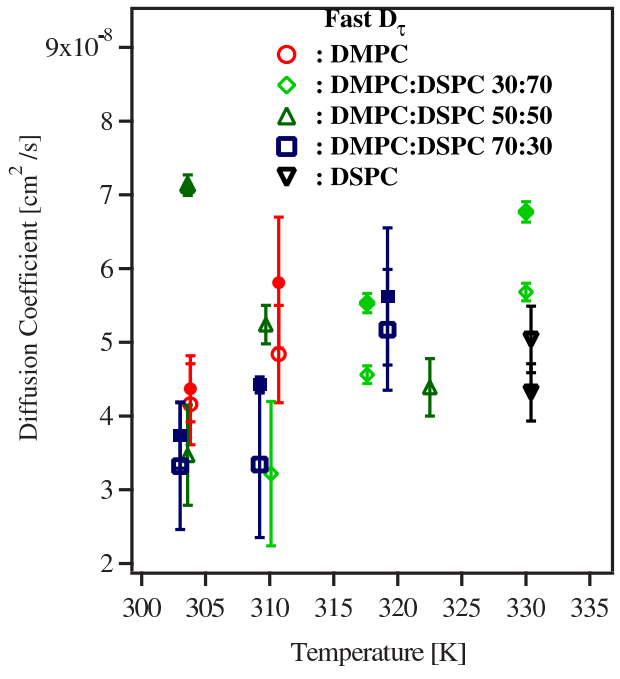

(a)

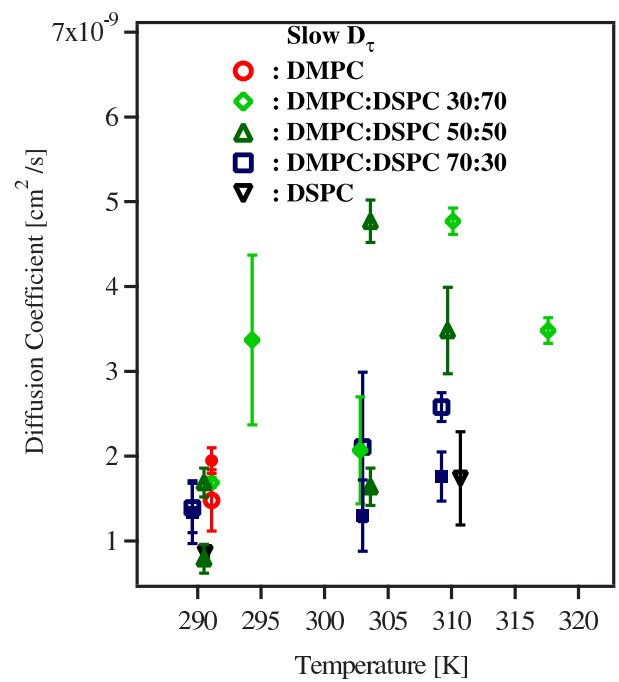

(b)

Figure 4.20: Diffusion coefficient of DiIC18 in various DMPC:DSPC mixtures of a fast component (a) and a slow component (b) as a function of temperature. The open symbols represent measurements performed in the croscorrelation mode, whereas the filled symbols in the autocorrelation mode. 
Table 4.6: Average translational diffusion coefficients of a slow component, $\left\langle D_{\tau_{\text {slow }}}\right\rangle$, and of a fast component, $\left\langle D_{\tau_{\text {fast }}}\right\rangle$, in DMPC:DSPC lipid mixtures at various temperature measured by FCS. $\sigma_{\left\langle D_{\tau}\right\rangle}$ is the standard deviation of an average $\left\langle D_{\tau}\right\rangle$. Experiments were recorded in crosscorrelation mode. (-) means that at given temperature, a gel or a fluid phase does not exist. Temperatures with $*$ are close to the melting temperatures of DMPC and DSPC.

\begin{tabular}{|c|c|c|c|c|c|}
\hline DMPC:DSPC & Temp. & $\left\langle D_{\tau_{\text {fast }}}\right\rangle$ & $\sigma_{\left\langle D_{\tau_{\text {fast }}}\right\rangle}$ & $\left\langle D_{\tau_{\text {slow }}}\right\rangle$ & $\sigma_{\left\langle D_{\tau_{\text {slow }}}\right\rangle}$ \\
a molar fraction & $\mathrm{K}$ & $\cdot 10^{-8} \frac{\mathrm{cm}}{\mathrm{s}}$ & $\cdot 10^{-8} \frac{\mathrm{cm}}{\mathrm{s}}$ & $\cdot 10^{-9} \frac{\mathrm{cm}^{2}}{\mathrm{~s}}$ & $\cdot 10^{-9} \frac{\mathrm{cm}}{\mathrm{s}}$ \\
\hline \multirow{3}{*}{$100: 0$} & 291.1 & - & - & 1.48 & 0.36 \\
& $296.6^{*}$ & 5.64 & 1.86 & 1.87 & 0.46 \\
& 303.8 & 4.16 & 0.55 & - & - \\
& 310.7 & 4.84 & 0.66 & - & - \\
\hline \multirow{3}{*}{$30: 70$} & 291.0 & - & - & 1.69 & 0.58 \\
& 310.1 & 3.22 & 0.98 & 4.77 & 1.55 \\
& 317.6 & 4.56 & 0.12 & 3.48 & 0.15 \\
& 330.0 & 5.68 & 0.12 & - & - \\
\hline \multirow{5}{*}{$50: 50$} & 290.5 & - & - & 0.79 & 0.17 \\
& 303.6 & 3.47 & 0.68 & 0.83 & 0.25 \\
& 309.7 & 5.24 & 0.26 & 1.03 & 0.51 \\
& 322.5 & 4.39 & 0.39 & - & - \\
\hline \multirow{3}{*}{$70: 30$} & 289.3 & - & - & 1.39 & 0.29 \\
& 303.0 & 3.32 & 0.86 & 2.11 & 0.88 \\
& 309.2 & 3.34 & 0.99 & 2.58 & 0.17 \\
& 319.2 & 5.17 & 0.82 & - & - \\
\hline \multirow{3}{*}{$0: 100$} & 290.6 & - & - & 0.86 & 0.05 \\
& 310.7 & - & - & 1.74 & 0.55 \\
& $328.2 *$ & 3.76 & 2.54 & 2.5 & 0.12 \\
& 330.4 & 5.04 & 4.52 & - & - \\
\hline
\end{tabular}

Slower translational diffusion coefficient was observed as the amount of gel increases (see Figure 4.18, Figure 4.19 and Figure 4.20).

The shape of the cross-correlation profiles for phase coexistent regions differs from those performed in a pure gel or a pure fluid phase.

In a gel-fluid coexistence region, diffusion of the fast component decreases (e.g. 3.22 . $10^{-8} \mathrm{~cm}^{2} / s$ for DMPC:DSPC 30:70 at $310.1 \mathrm{~K}, f_{l}=18 \%$ ) and diffusion of the slow component increases (e.g. $4.77 \cdot 10^{-9} \mathrm{~cm}^{2} / \mathrm{s}$ for DMPC:DSPC $30: 70$ at $310.1 \mathrm{~K}, f_{g}=92 \%$ ) in comparison to a pure fluid $\left(4.84 \cdot 10^{-8} \mathrm{~cm}^{2} / \mathrm{s}\right.$ for DMPC at $310.7 \mathrm{~K}$ ) and a pure gel $\left(1.74 \cdot 10^{-9} \mathrm{~cm}^{2} / \mathrm{s}\right.$ for DSPC at $\left.310.7 \mathrm{~K}\right)$ diffusion coefficient at the same temperature.

Lowering of a fast diffusion constant and increasing of a slow diffusion constant was ob- 
Table 4.7: Average translational diffusion coefficients of a slow component, $\left\langle D_{\tau_{\text {slow }}}\right\rangle$, and of a fast component, $\left\langle D_{\tau_{\text {fast }}}\right\rangle$, in DMPC:DSPC lipid mixtures at various temperature measured by FCS. $\sigma_{\left\langle D_{\tau}\right\rangle}$ is the standard deviation of an average $\left\langle D_{\tau}\right\rangle$. Experiments were recorded in autocorrelation mode. (-) means that at given temperature, a gel or a fluid phase does not exist. Temperatures with $*$ are close to the melting temperatures of DMPC and DSPC.

\begin{tabular}{|c|c|c|c|c|c|}
\hline DMPC:DSPC & Temp. & $\left\langle D_{\tau_{\text {fast }}}\right\rangle$ & $\sigma_{\left\langle D_{\tau_{\text {fast }}}\right\rangle}$ & $\left\langle D_{\tau_{\text {slow }}}\right\rangle$ & $\sigma_{\left\langle D_{\tau_{\text {slow }}}\right\rangle}$ \\
a molar fraction & $\mathrm{K}$ & $\cdot 10^{-8} \frac{\mathrm{cm}^{2}}{\mathrm{~s}}$ & $\cdot 10^{-8} \frac{\mathrm{cm}}{\mathrm{s}}$ & $\cdot 10^{-9} \frac{\mathrm{cm}}{\mathrm{s}}$ & $\cdot 10^{-9} \frac{\mathrm{cm}}{\mathrm{s}}$ \\
\hline \multirow{3}{*}{$100: 0$} & 291.1 & - & - & 1.95 & 0.15 \\
& $296.6^{*}$ & 4.93 & 0.25 & 1.60 & 0.65 \\
& 303.8 & 4.37 & 0.45 & - & - \\
& 310.7 & 5.81 & 0.89 & - & - \\
\hline \multirow{3}{*}{$30: 70$} & 291.0 & - & - & 2.49 & 0.67 \\
& 310.1 & 4.55 & 0.76 & 3.37 & 1.00 \\
& 317.6 & 5.53 & 0.13 & 2.07 & 0.63 \\
& 330.0 & 6.77 & 0.14 & - & - \\
\hline \multirow{3}{*}{$50: 50$} & 290.5 & - & - & 0.79 & 0.17 \\
& 303.6 & 7.13 & 0.14 & 1.64 & 0.22 \\
& 309.7 & not measured & not measured & not measured & not measured \\
& 322.5 & not measured & not measured & - & - \\
\hline \multirow{3}{*}{$70: 30$} & 289.3 & - & - & 1.34 & 0.37 \\
& 303.0 & 3.74 & 0.45 & 1.30 & 0.42 \\
& 309.2 & 4.42 & 0.11 & 1.76 & 0.29 \\
& 319.2 & 5.62 & 0.93 & - & - \\
\hline \multirow{3}{*}{$0: 100$} & 290.6 & - & - & not measured & not measured \\
& 310.7 & - & - & not measured & not measured \\
& $328.2 *$ & 3.76 & 0.25 & 2.5 & 0.12 \\
& 330.4 & 4.32 & 0.39 & - & - \\
\hline
\end{tabular}

served for every mixture when changing the temperature (refer to Table 4.6 and Figure 4.18, Figure 4.25, Figure 4.26, Figure 4.27 and Fig 4.19). This may indicate that there are gel microdomains inside fluid domains, and fluid microdomains inside gel domains. To check this hypothesis a quantitatively comparison of FCS data with Monte Carlo simulations was performed.

When calculating the diffusion coefficient, there are several sources of errors. The diffusion coefficient of a lipid can be only calculated by comparison to an experiment with rhodamine $6 \mathrm{G}$. The focal radius and the diffusion time of rhodamine $6 \mathrm{G}$ varies between individual measurements but averages a value of $377 \mathrm{~nm}$. Thus, the error for the diffusion coefficient of lipids accumulates the error from both experiments: rhodamine and lipids (refer to Eq. 4.4). 


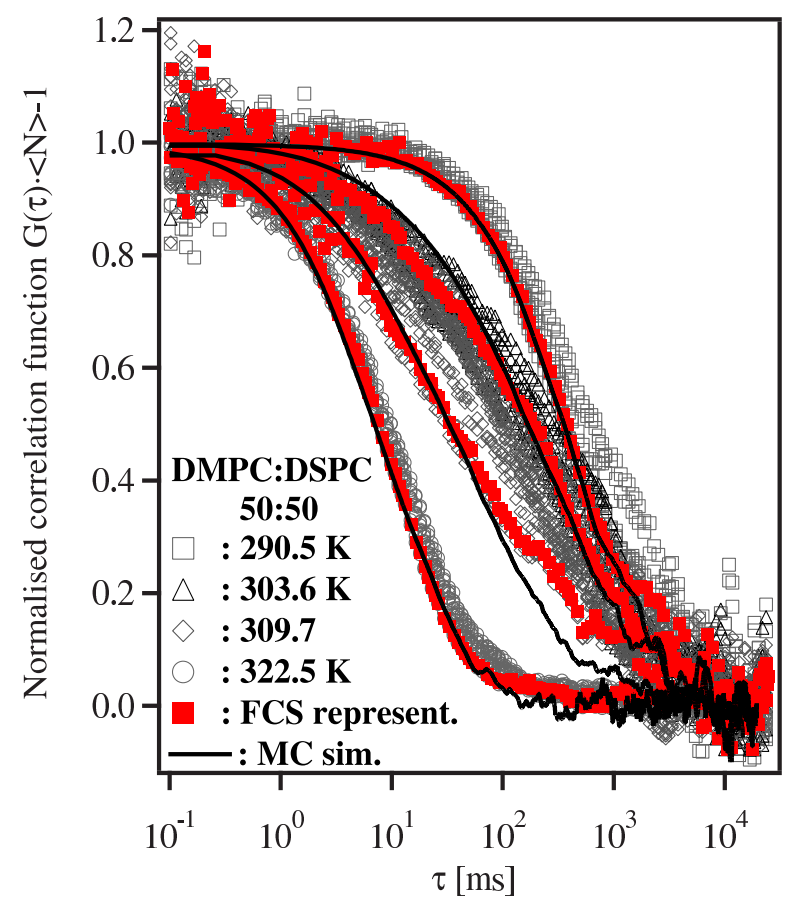

Figure 4.21: Normalised crosscorrelation profiles of DiIC18 diffusing in DMPC:DSPC 70:30 membranes at various temperatures. Gray symbols represent FCS measurements, red filled symbols represent curves which were compared with MC simulations (black curves).

This is why, for the comparison of FCS data with MC simulations, the diffusion coefficients for lipids were corrected with the mean value of the focal radius.

\subsubsection{Monte Carlo simulations}

We wanted to understand domain formation with the help of Monte Carlo simulations based on the two-state Ising model [76, 105, 110, 109, 111]. Therefore, FCS results were analysed with Monte Carlo simulations which make use of thermodynamical properties of the lipid system. All of MC simulations were performed with exactly the same input parameters. Monte Carlo simulations led to the simulation of diffusion processes in membranes (see Section 2.4.4) (refer to Figure 4.22). The Monte Carlo simulations were done on a $70 \times 70$ lattice. Calculations on larger lattice sizes $(80 \times 80,100 \times 100$ and $200 \times 200)$ were found to give the same results within statistical error of $15 \%$.

In Figure 4.23, MC snapshots in the gel phase, the gel and fluid coexistence phase and the fluid phase can be seen.

The red symbols correspond to the gel domains and the green symbols to the fluid do- 


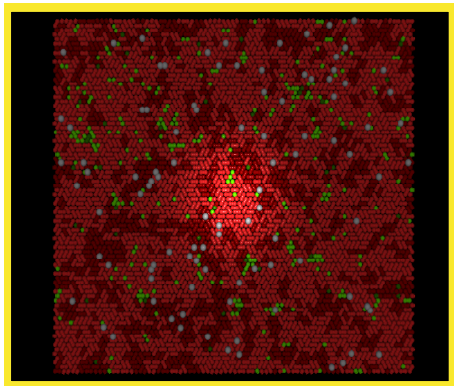

$289.3 \mathrm{~K}$

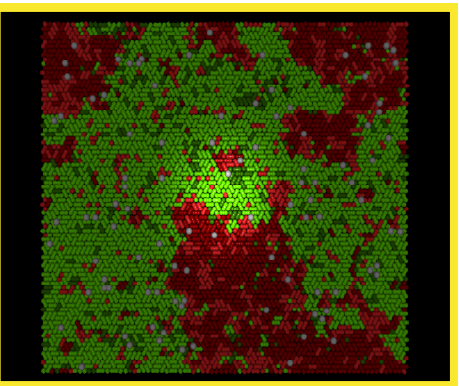

$304 \mathrm{~K}$

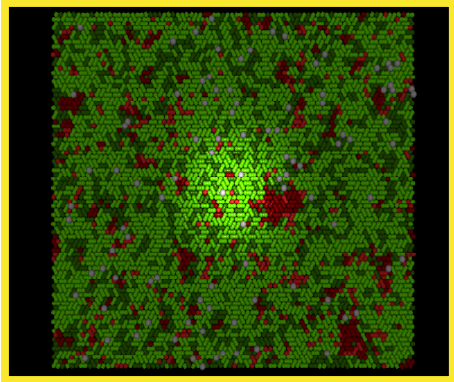

$312 \mathrm{~K}$

Figure 4.22: MC snapshots performed using a DMPC:DSPC 70:30 mixture at various temperatures (289.3 K, $304 \mathrm{~K}, f_{g}=33 \% ; 312 \mathrm{~K}$ ). The red symbols correspond to the gel domains and the green symbols to the fluid domains. The light green and red symbols correspond to the DMPC and the dark green and red symbols to DSPC. The connection between points represents the chemical bond. 100 chains were marked, which are indicated with white spheres. The focus is indicated as a light spherical shadow. The matrix size was $70 \times 70$.

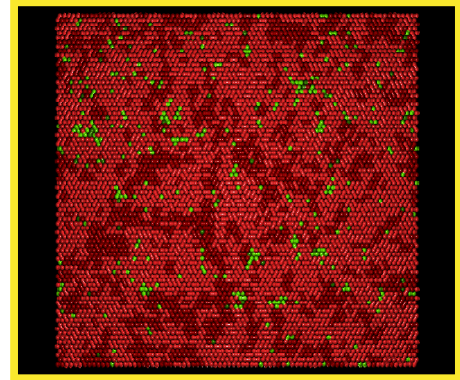

$289.3 \mathrm{~K}$

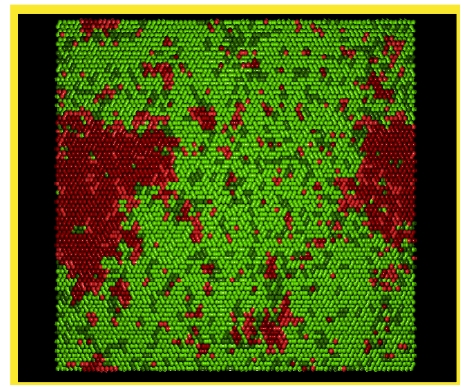

$309.2 \mathrm{~K}$

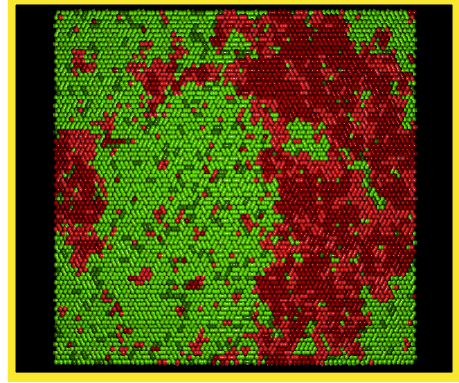

$303 \mathrm{~K}$

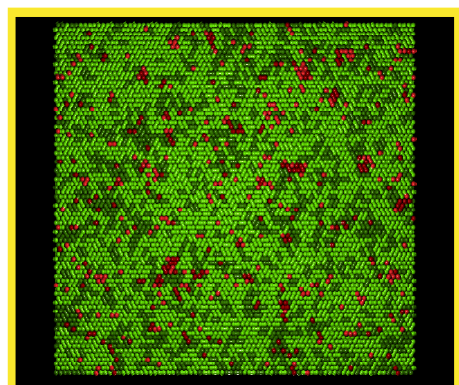

$319.2 \mathrm{~K}$

Figure 4.23: MC snapshots performed using a DMPC:DSPC 70:30 mixture in the gel phase (289.2 K), or in the gel and fluid coexistence regime (303 K, $\left.f_{g}=40.4 \% ; 309.2 \mathrm{~K}, f_{g}=24 \%\right)$ and in the fluid phase $(319.2 \mathrm{~K})$ at temperatures corresponding to temperatures at which FCS measurements were performed. The red symbols correspond to the gel domains and the green symbols to the fluid domains. The light green and red symbols correspond to the DMPC and the dark green and red symbols to DSPC. The connection between points represents the chemical bond. 100 chains were marked, which are indicated with white spheres. The matrix size was $70 \times 70$.

mains. The lighter shades of each colour correspond to the shorter chain lipid species (DMPC) and the darker shades to the longer lipid chain (DSPC). Huge gel and fluid domains which are not homogenous can be seen. The MC simulations of the fluorescent traces of single labelled 
chains show fluid microdomains inside gel parts and vice versa.

Seeger, from Membrane Thermodynamics and Biophysics Group of MPI for biophysical Chemistry has performed Monte Carlo simulations at the same temperatures as the experiments were performed. With MC, simulation of the emission of fluorescence by a marked lipid chain and thereby diffusion can be performed. The MC simulations have $5 \cdot 10^{5}$ steps. The various Monte Carlo fluorescence traces at different temperatures are graphically depicted in a Figure 4.24.

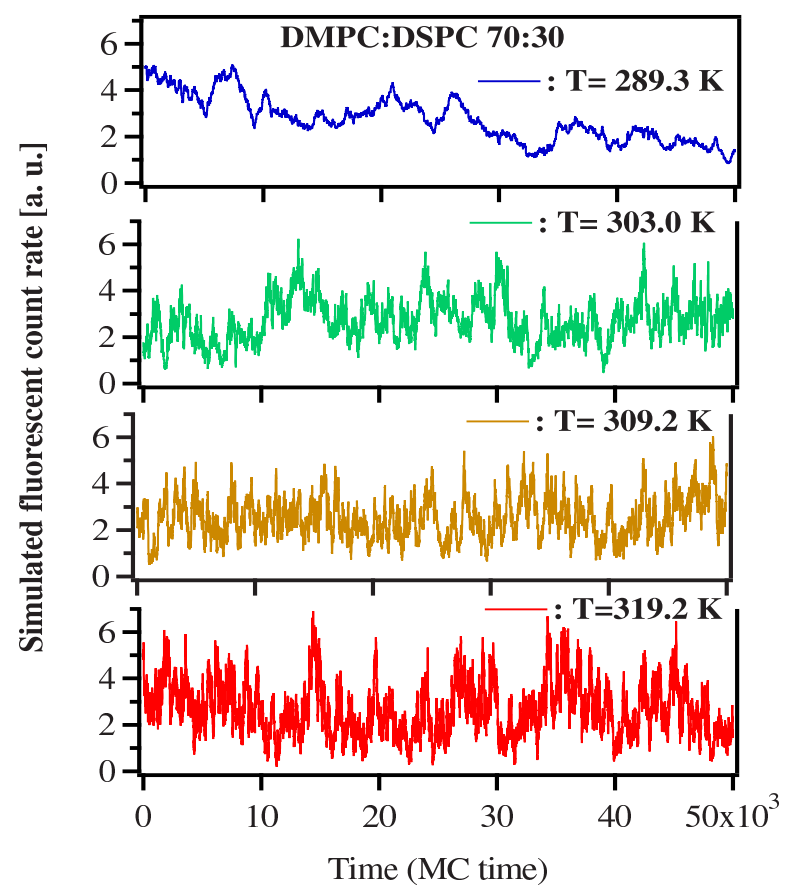

Figure 4.24: The MC fluorescent noise for a DMPC:DSPC 70:30 mixture at various temperatures.

As seen the slowest fluctuations occur in a gel phase (at $289.3 \mathrm{~K}$ ), the superposition of slow and fast fluctuations occur in coexistence phases (at $303 \mathrm{~K}$ and $309.2 \mathrm{~K}$ ) and the faster fluctuations occur in a fluid phase (at $319.2 \mathrm{~K}$ ). These simulations are in a good agreement with the expectations of fast fluctuations in a fluid phase whereas it is possible a lot molecular motions. Slow fluctuations occur in a gel phase whereas the molecular motion is reduced, because of closer molecular packing. In the intermediate temperature regime, (see Figure 4.24 at $303 \mathrm{~K}$ ), one can provide a line corresponding to the slow fluctuations on which fast fluctuations occur. Slow and fast diffusing fluorescent molecules with FCS are shown visually in Figure 4.2 in Section 4.1. 
The Monte Carlo procedure does not provide a realistic time scale. The time scales can be only determined by comparison with the FCS experiment. Only the samples measured in crosscorrelation mode, which reduces noise on fast time scales, were compared with MC simulations. MCS are in quantitative agreement with the observed data (see Figure 4.25, Figure 4.26, Figure 4.27, and Figure 4.20).

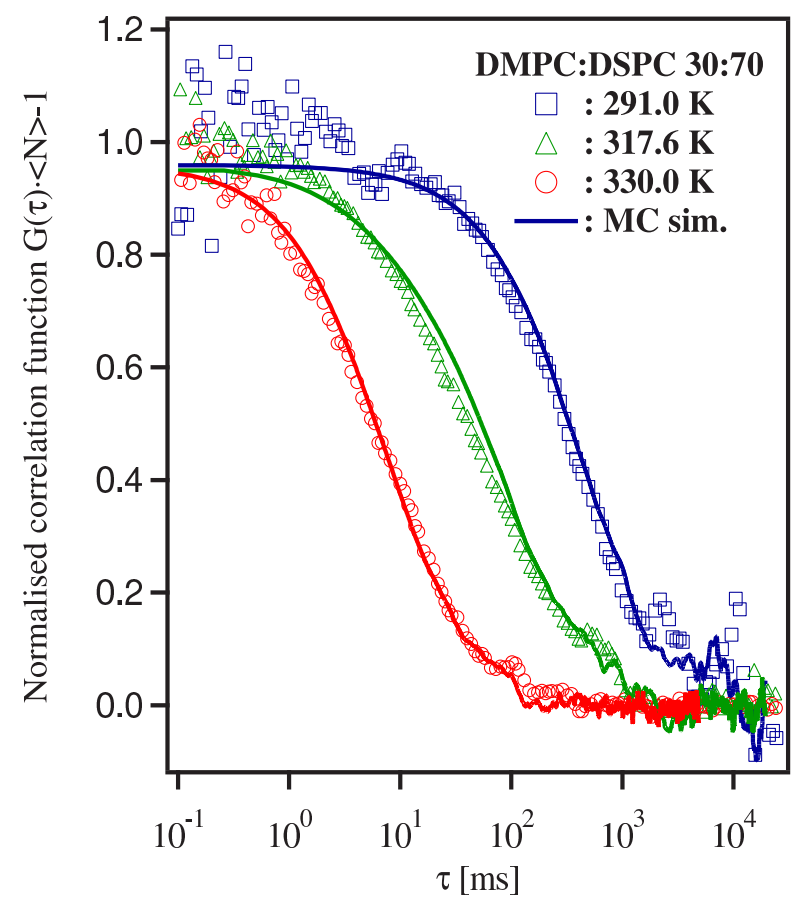

Figure 4.25: Normalised cross-correlation profiles of DiIC18 diffusing in a DMPC:DSPC 30:70 mixture at various temperatures. Markers represent FCS measurements. Solid lines are predictions from Monte Carlo simulations.

MCS provides a good representation of the experimental curves in all temperatures for every mixture. There are small deviations (e.g. for the mixture DMPC:DSPC 50:50, see Figure 4.26), which are the result of inaccurate temperature measurements in the FCS experiments. The values of the measured temperatures on the coverslips, the sample holder and on the objective differs quite a lot (even several K). The different temperatures refer to different amounts of gel and fluid phases than expected from the phase diagram. (refer to Table 4.2). Also, the amount of detected gel and fluid phase depends on the partition of the marker in both phases. DiIC18 has the best partition in a gel and in a fluid phase from the available dyes (see Section 3.2.1).

Another observed phenomena, is the pronounced quenching of the DiIC18 in the gel . This 


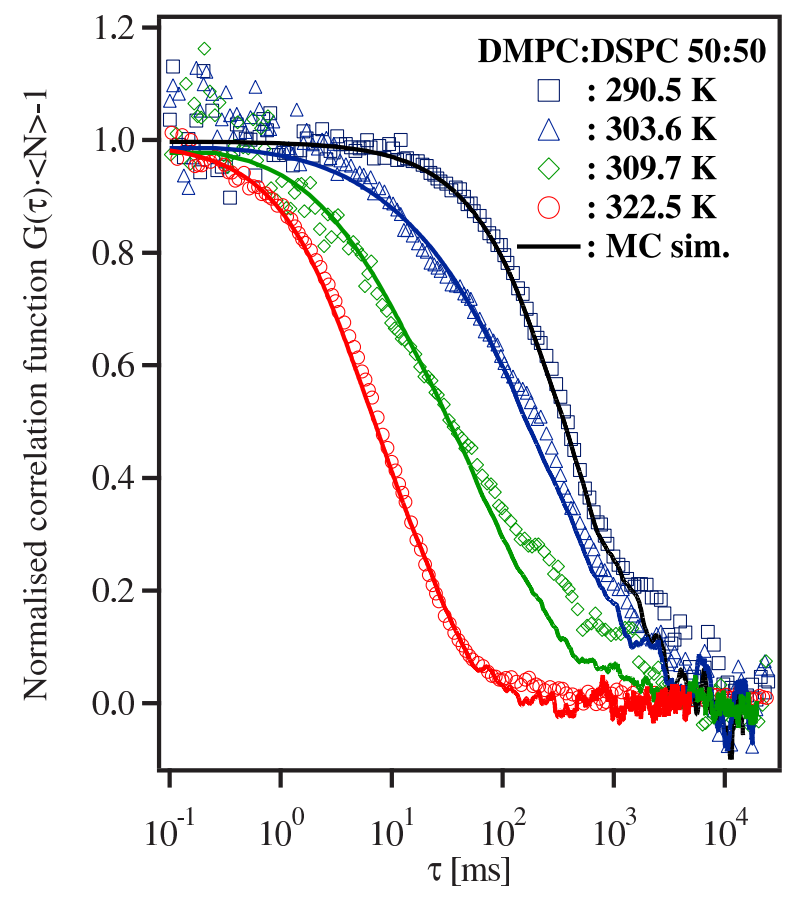

Figure 4.26: Normalised cross-correlation profiles of DiIC18diffusing in a DMPC:DSPC 50:50 mixture at various temperatures. Markers represent FCS measurements. Solid lines are predictions from Monte Carlo simulations.

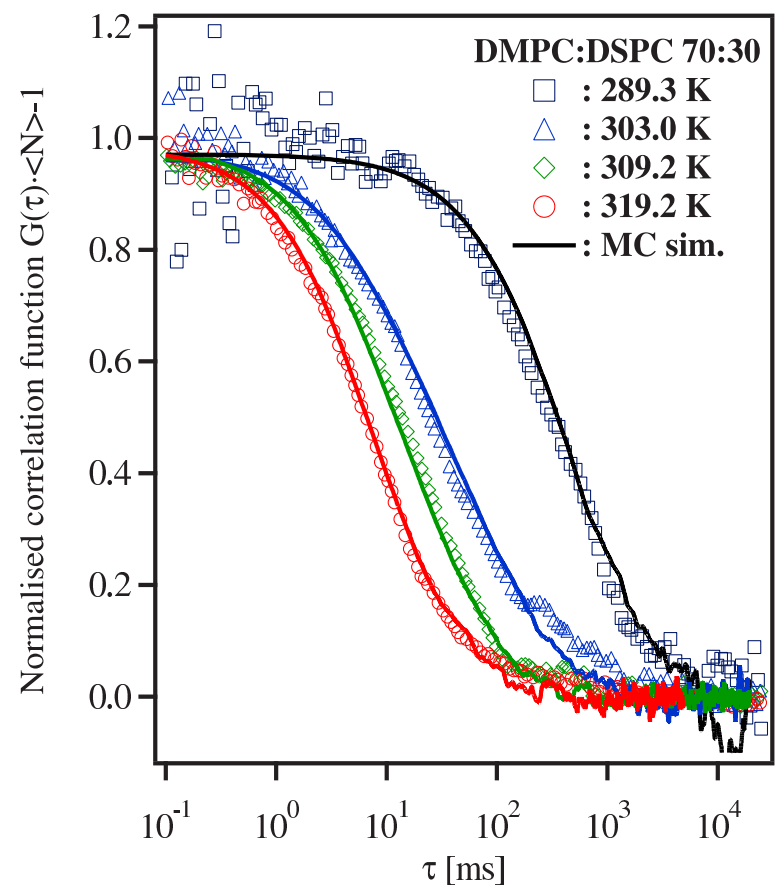

Figure 4.27: Normalised cross-correlation profiles of DiIC18diffusing in a DMPC:DSPC 70:30 mixture at various temperatures. Markers represent FCS measurements. Solid lines are predictions from Monte Carlo simulations. 
indicates that clustering of label in the gel domains may be occurring. This appears as a reduced count rate per molecule. When the same probe was heated the count rate per molecule increased again. The marker clusters diffuse slower since they are bigger complexes than a single dye molecule (refer to Figure 4.26).

There are also small abbreviations in MC simulations on a slow time scales (s). At the end of the MC simulated correlation curve is noise which reduced with longer simulation times (more MC steps) or with matrix smaller sizes. However, increasing the number of MC cycles has the disadvantage that the time of the simulations increasing rapidly.

Monte Carlo simulations are consistent with FCS measurements, therefore information about the domain and microdomain formation as a result of adjacent lipid interactions was obtained. On MC snapshots, gel and fluid macroscopic and microscopic domains in coexisting phases were seen. Also, the shape of FCS profiles predicts microdomain formation in the intermediate temperature regime (as discussed in Section 4.3 and 4.4.1.). The diffusion coefficient in fluid domains is lowered since there are also gel microdomains, whereas the diffusion coefficient in gel domains increases because of the presence of fluid microdomains. The good agreement between theoretical and experimental curves proves existence of microheterogenities in membranes. Therefore, the two-component fitting procedure, based on macroscopic phase separation, does not give the correct interpretation of diffusion processes occurring in the gel and fluid domains in a coexistent region.

Since Monte Carlo simulations make use of thermodynamical properties of the lipid system, they give us information not only about the structure of lipid membranes, but also can help with interpretation of their kinetics. MC has the advantage that they give us information about diffusion times and also about relaxation times. The relaxation time, $\tau_{r} \propto T^{3} \cdot c_{p}$, describes how quickly domains grow and disappear (i.e., how fast and often lipids change the state gel-fluid [51]). The highest fluctuations are at the melting transition and on the domain boundaries.

\subsection{Ionic strength dependence of diffusion}

The effect of electrostatic interactions of ions with phospholipids has previously been studied by calorimetry [17; 116]. Chapman has studied the influence of divalent cations $\mathrm{Ca}^{2+}, \mathrm{Mg}^{2+}$ and $\mathrm{UO}_{2}{ }^{2+}$ on the phase transition in 1,2-diplalmitoyl lecithin and ox brain phosphatidylser- 
ine. Taylor has investigated the modification of the phase transition of DPPC in the presence of mono- di- $\left(\mathrm{Ca}^{2+}, \mathrm{Mg}^{2+}\right)$ and tri-valent $\left(\mathrm{Pr}^{3+}, \mathrm{Eu}^{3+}, \mathrm{Gd}^{3+}\right)$ ions. They found that ions shift the melting transition to higher temperatures. These studies encourage us to further investigate the structure and the dynamics of uncharged membranes (POPC) in the presence of different ions. Since biological membranes are surrounded by an aquas buffer containing various ions like $\mathrm{Na}^{+}, \mathrm{K}^{+}, \mathrm{Ca}^{2+}, \mathrm{Mg}^{2+}$ and $\mathrm{Cl}^{-}$, the interactions of these ions were investigated with the zwitterionic lipid, POPC ${ }^{10}$. Ions $\mathrm{Na}^{+}$and $\mathrm{K}^{+}$are involved in the $\left(\mathrm{Na}^{+}, \mathrm{K}^{+}\right)$ATPase whereas ions $\mathrm{Ca}^{2+} \mathrm{Mg}^{2+}$ involved in $\left(\mathrm{Ca}^{2+}, \mathrm{Mg}^{2+}\right)$ ATPase play an important role as neurotransmitters in the nervous system. This study combines FCS experiments with Molecular Dynamic simulations that provides a new picture on the influence of various salt concentrations of $\mathrm{NaCl}$ on lateral diffusion of individual lipids in pure POPC membranes [11].

\subsubsection{Calorimetric measurements}

POPC has a melting temperature below the freezing point of water $\left(T_{m}=269.7 \mathrm{~K}\right.$, see Figure 4.28). Therefore, it has been measured in a capillary cell with a smaller volume. This was inserted in an ethylenglycol water solution 40:60 mass \%. MLVs ${ }^{11}$ of POPC have been measured in the presence of different $\mathrm{NaCl}, \mathrm{KCl}, \mathrm{MgCl}_{2}$ and $\mathrm{CaCl}_{2}$ concentrations. The scan rate was $5 \mathrm{~K} / \mathrm{hr}$. A melting enthalpy of $19.8 \mathrm{~kJ} / \mathrm{mol}$ was determined.

The heat capacity profiles of POPC with $\mathrm{NaCl}$ are showed in Figure 4.28, with $\mathrm{KCl}$ in Fig 4.29, with $\mathrm{CaCl}_{2}$ in Figure 4.30 and with $\mathrm{MgCl}_{2}$ in Figure 4.31 .

In the case of POPC with $\mathrm{NaCl}$ and $\mathrm{KCl}$, a small but measurable effect on the heat capacity curve can be seen. In the case of $\mathrm{MgCl}_{2}$ and $\mathrm{CaCl}_{2}$ this effect is clearly visible. Increasing the ionic strength changes the shape of the calorimetric profiles. The ionic strength, $c$, depends on the concentration of positive $\left(C_{+}^{0}\right)$ and negative $\left(C_{-}^{0}\right)$ ions and their valence, $z$, as given by [114],

$$
c=\frac{1}{2} \sum_{i}\left(z_{i+}^{2} \cdot C_{i+}^{0}+z_{i-}^{2} \cdot C_{i-}^{0}\right) .
$$

The interactions of the divalent ions, $\mathrm{Ca}^{2+}$ and $\mathrm{Mg}^{2+}$, with membranes can be observed at lower concentrations than in the case of monovalent ions, $\mathrm{Na}^{+}$and $\mathrm{K}^{+}$(refer to Figure 4.28 and Figure 4.29). In the presence of ions, the value of the maximum heat capacity decreases and the overall profile is shifted to higher temperatures. The reduction in the shifted original

\footnotetext{
10 16:0-18:1 PC, 1-palmitoyl-2-oleoyl-sn-glycero-3-phosphocholine.

${ }^{11}$ Multilamellar Vesicles.
} 


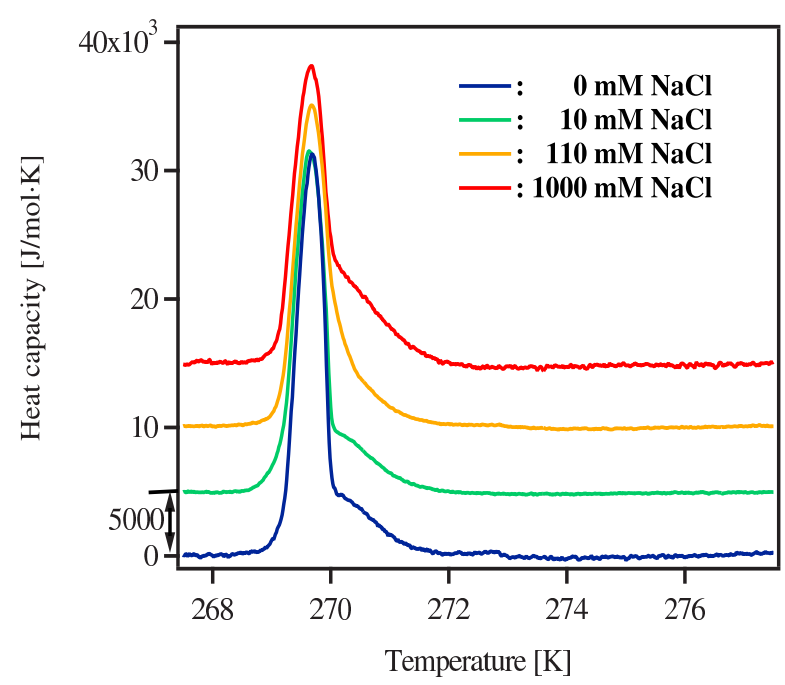

Figure 4.28: Calorimetric profiles of POPC multilayers at different $\mathrm{NaCl}$ concentrations.

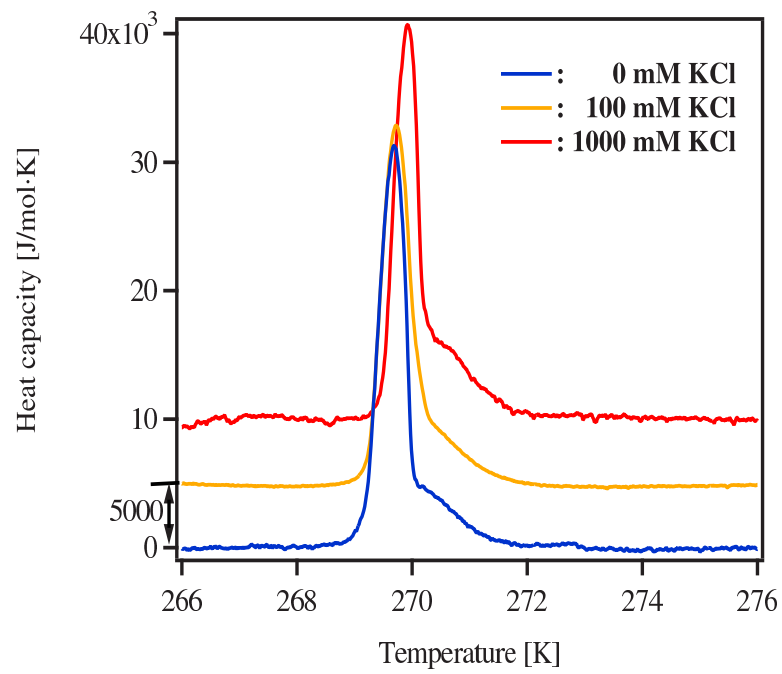

Figure 4.29: Calorimetric profiles of POPC multilayers at different $\mathrm{KCl}$ concentrations.

transition peak and the appearance of a new peak at a higher temperature was found for divalent ions. The effect of $\mathrm{Ca}^{2+}$ is even stronger than that produced by similar concentrations of $\mathrm{Mg}^{2+}$. The shift in the melting temperatures indicates that these ions $\left(\mathrm{Na}^{+}, \mathrm{K}^{+}, \mathrm{Ca}^{2+}\right.$, $\mathrm{Mg}^{2+}$ ) prefer to interact with the gel ordered phase of the lipid membrane. Interactions of ions on a sodium example with FCS were studied and analysed with MD simulations in a collaboration with Böckmann and Grubmüller of the Theoretical Molecular Biophysics Group of of MPI for biophysical Chemistry. 


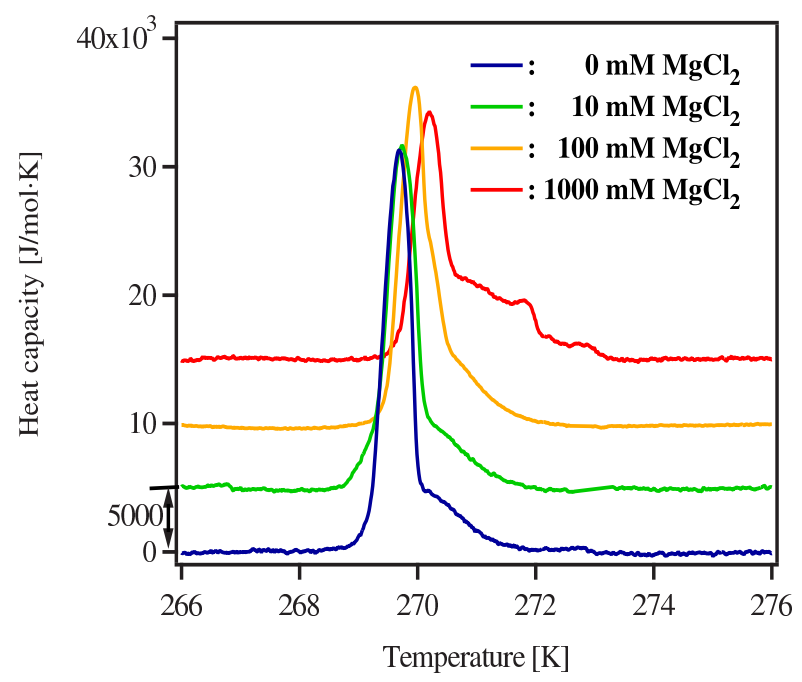

Figure 4.30: Calorimetric profiles of POPC multilayers at different $\mathrm{MgCl}_{2}$ concentrations.

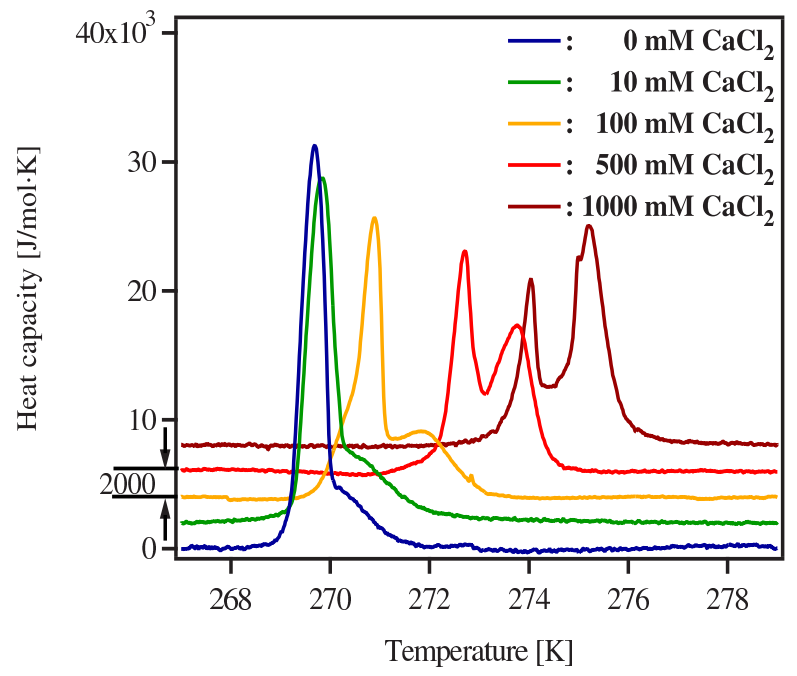

Figure 4.31: Calorimetric profiles of POPC multilayers at different $\mathrm{CaCl}_{2}$ concentrations.

\subsubsection{FCS experiment}

For the FCS measurements, dry POPC membranes $(20 \mu \mathrm{l}$ of $10 \mathrm{mM}$ lipid soluted in a Dichloromethane:Methanol 2:1) on quartz were hydrated with $30 \mu \mathrm{l}$ of a buffer with various $\mathrm{NaCl}$ concentrations and heated above the melting temperature of POPC. Samples were then equalise for half an hour in order to ensure complete hydration. As a diffusing particle, one of the following two fluorescence markers was used: TRITC DHPE ${ }^{12}$ (see Figure 3.9), with

\footnotetext{
${ }^{12} \mathrm{~N}$-(6-tetramethylrhodaminethiocarbamoyl)-1,2-dihexadecanoyl-sn-glycero-3-phosphoethanolamine, triethylammonium salt.
} 
a fluorescent labelled and charged headgroup or an uncharged BODIPY-C12-HPE ${ }^{13}$ with a fluorescent labelled the second chain (see Figure 3.12). Because of the charge on TRITC DHPE, it may have a different ionic strength dependence than zwitterionic lipids. However it has similar chain composition as POPC. The second marker has longer chains that POPC which can reduce the diffusion coefficient.

The fluorescence signal was detected by two avalanche photodiodes (APD) and correlated on-line in crosscorrelation or autocorrelation mode. Measurements were performed with a pinhole $100 \mu \mathrm{m}$ and OD filter 3. Measurements for POPC in bidistilled water and POPC with different $\mathrm{NaCl}$ concentrations were performed, each at three different temperatures within the fluid phase. Each sample was measured at every temperature, approximately 20 times with a duration $120 \mathrm{~s}$ and $300 \mathrm{~s}$ for each measurement. Several samples, at several different locations were measured at every temperature. Time scales were calibrated with rhodamine $6 \mathrm{G}$ in bidistilled water at $296 \mathrm{~K}$.

Cross-correlation profiles could be fitted assuming a one-component diffusion rate (refer to Eq. 4.3). The temperature dependence diffusion of BODIPY-C12-HPE and TRITC DHPE in planar multilamellar membranes of POPC on quartz, hydrated by $110 \mathrm{mM} \mathrm{NaCl}$ is showed in Figure 4.32 and in Figure 4.33 respectively. Time scales were calibrated with rhodamine $6 \mathrm{G}$ in bidistilled water at $296 \mathrm{~K}$.

The fluorescence traces, analysed by the correlation function for several salts concentrations, are shown in Figure 4.34 for BODIPY-C12-HPE and in Figure 4.35 for TRITC DHPE.

The average values of translational diffusion coefficients for both modes, $\left\langle D_{\tau}\right\rangle=\frac{D_{\tau}}{n}$, over $n$ measurements and their standard deviations, $\sigma_{\left\langle D_{\tau}\right\rangle}$, are shown for BODIPY-C12-HPE in Table 4.8 and for TRITC DHPE in Table 4.9.

Figures with various salt concentrations (see Figure 4.34 for BODIPY-C12-HPE and Figure 4.35 for TRITC DHPE) presents ionic strength dependence for both labels. Increasing the sodium chloride concentration was found to decrease the self-diffusion of POPC lipids within the bilayer (refer to Tables 4.8 and 4.9).

For TRITC DHPE at $\sim 300 \mathrm{~K}$, it was observed that the translational diffusion coefficient of $\left\langle D_{\tau}\right\rangle=7.75 \cdot 10^{-8} \mathrm{~cm}^{2} / \mathrm{s}$ in the absence of $\mathrm{NaCl}$ decreases to $\left\langle D_{\tau}\right\rangle=1.71 \cdot 10^{-8} \mathrm{~cm}^{2} / \mathrm{s}$ for $110 \mathrm{mM} \mathrm{NaCl}$ and to $\left\langle D_{\tau}\right\rangle=1.38 \cdot 10^{-8} \mathrm{~cm}^{2} / s$ for $5 \mathrm{M} \mathrm{NaCl}$ (refer to Table 4.9). In the

\footnotetext{
13-(4,4-difluoro-5,7-diphenyl-4-bora-3a, 4a-diaza-s-indacene-3-dodecanoyl)-1-hexadecanoyl-sn-glycero-3phosphoethanolamine.
} 


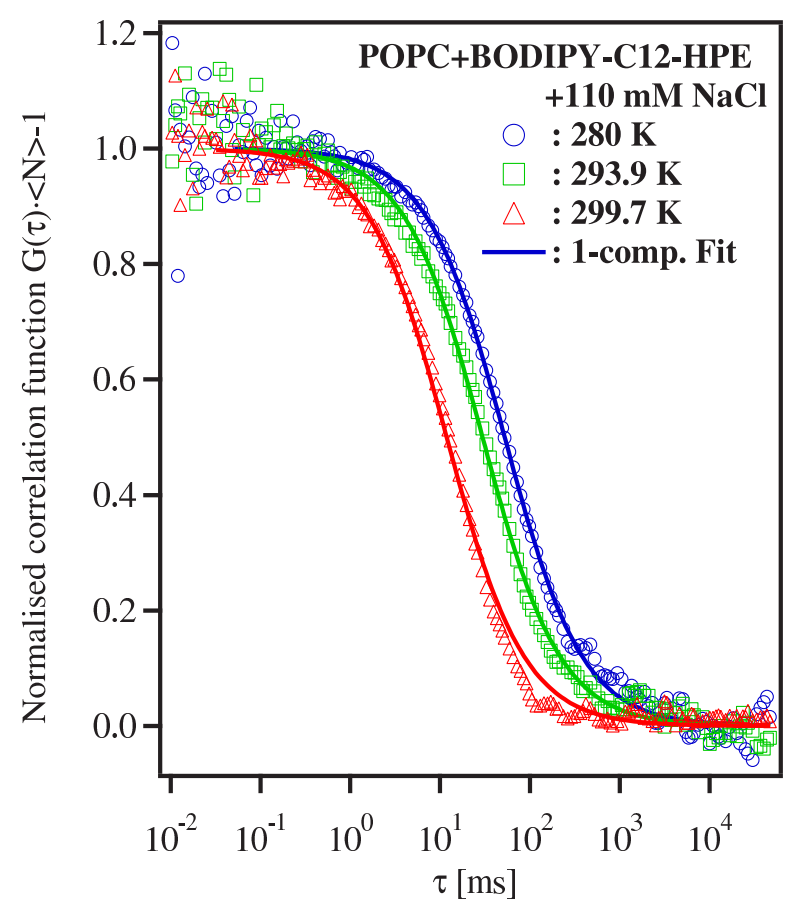

Figure 4.32: Normalised cross-correlation profiles of planar POPC membranes using BODIPY-C12-HPE hydrated by $110 \mathrm{mM} \mathrm{NaCl}$ at different temperatures. Markers represent FCS measurements, solid lines represent fits.

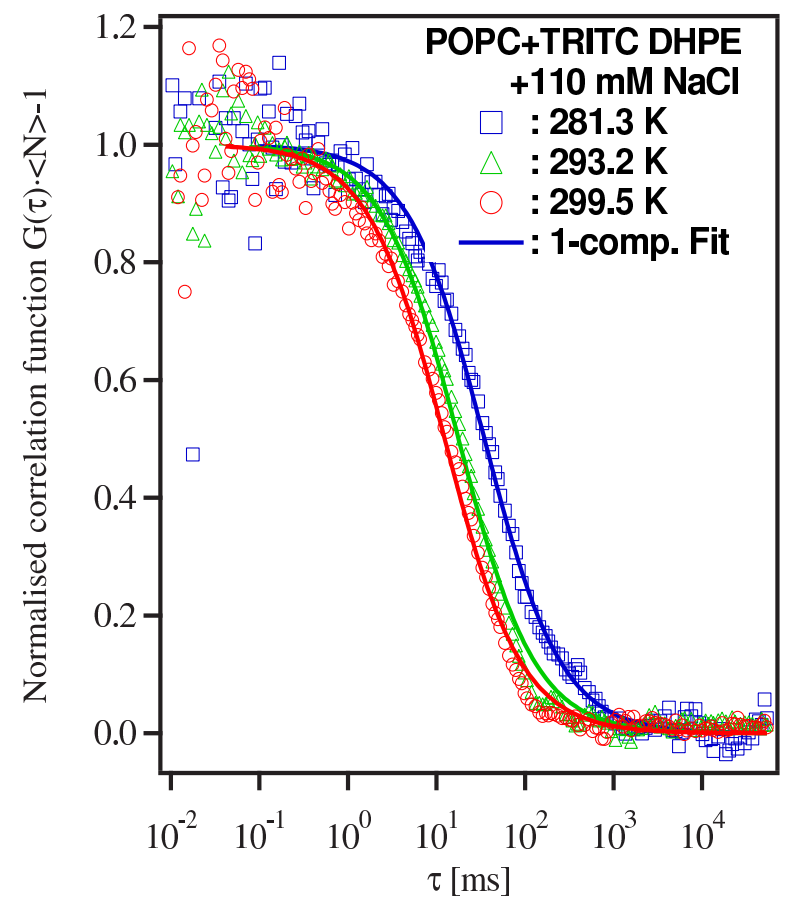

Figure 4.33: Normalised cross-correlation profiles of the TRITC DHPE diffusing in planar POPC membranes hydrated by $110 \mathrm{mM} \mathrm{NaCl}$ at different temperatures. Markers represent FCS measurements, solid lines represent fits. 


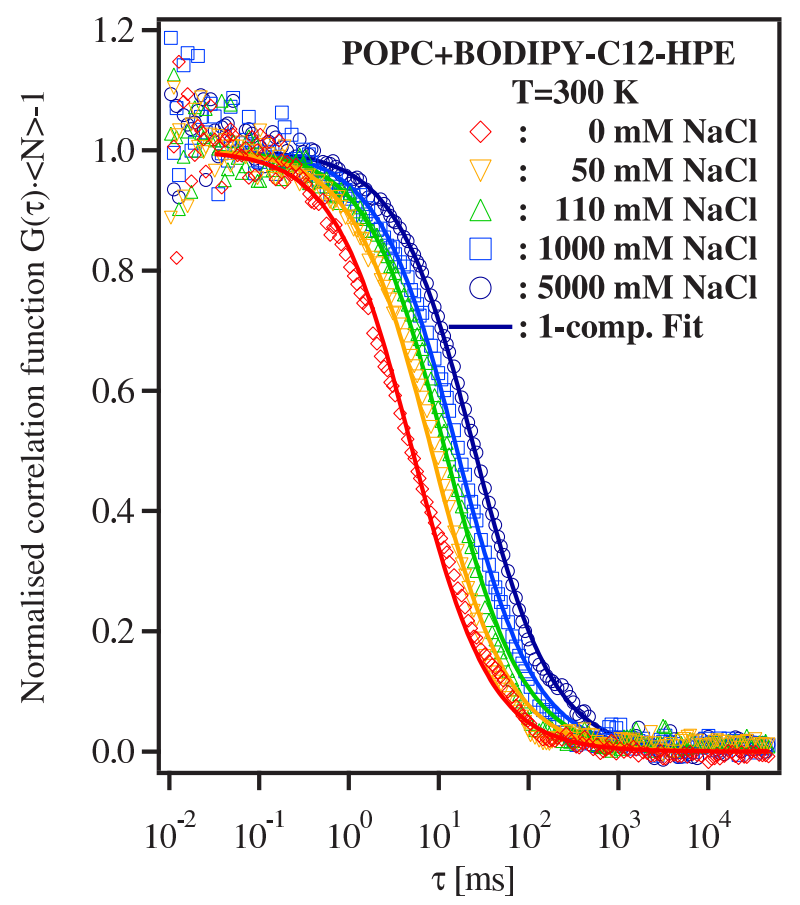

Figure 4.34: Normalised cross-correlation profiles of planar POPC membranes using BODIPY-C12-HPE as a function of $\mathrm{NaCl}$ concentration. Markers represent FCS measurements, solid lines represent fits.

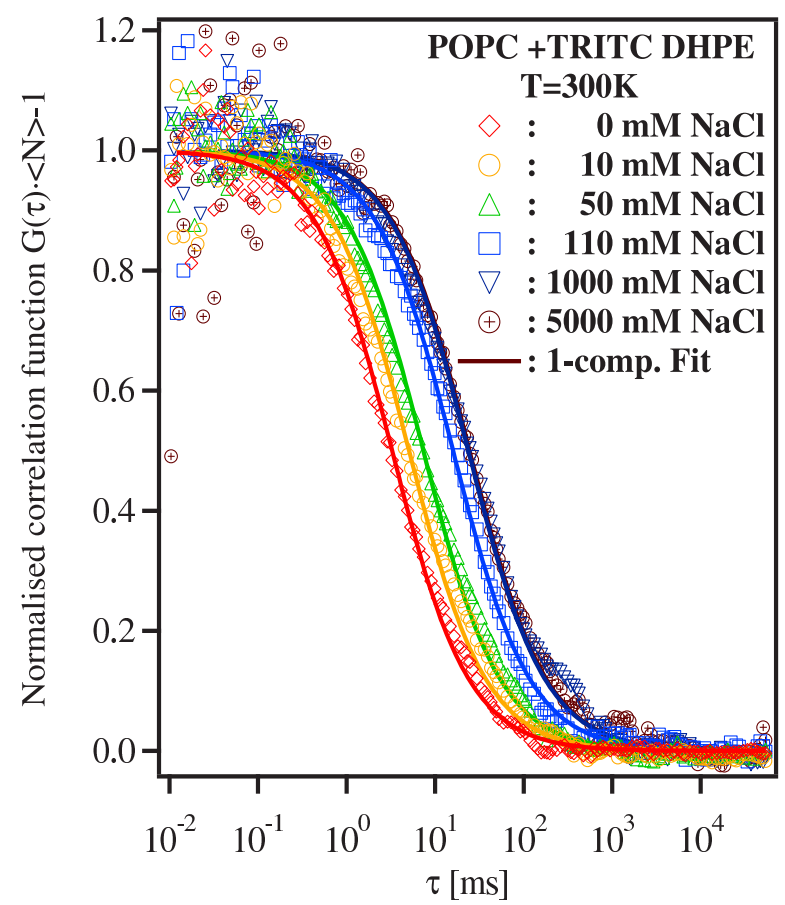

Figure 4.35: Normalised cross-correlation profiles of planar POPC membranes using TRITC DHPE as a function of $\mathrm{NaCl}$ concentration. Markers represent FCS measurements, solid lines represent fits. 
Table 4.8: Mean values of the diffusion coefficient, $\left\langle D_{\tau}\right\rangle$, of BODIPY-C12-HPE in POPC membranes at various temperatures with different $\mathrm{NaCl}$ concentrations recorded in crosscorrelation mode. $\sigma_{\left\langle D_{\tau}\right\rangle}$ is the standard deviation of $\left\langle D_{\tau}\right\rangle$.

\begin{tabular}{|c|c|c|c|}
\hline $\begin{array}{c}\mathrm{C}_{\mathrm{NaCl}} \\
\frac{M}{l}\end{array}$ & $\begin{array}{c}\text { Temp. } \\
\mathrm{K}\end{array}$ & $\begin{array}{c}\left\langle D_{\tau}\right\rangle \\
\cdot 10^{-8} \frac{\mathrm{cm}}{\mathrm{s}}\end{array}$ & $\begin{array}{c}\sigma_{\left\langle D_{\tau}\right\rangle} \\
\cdot 10^{-8} \frac{\mathrm{cm}}{\mathrm{s}}\end{array}$ \\
\hline \multirow{3}{*}{0} & 280.8 & 1.76 & 0.21 \\
& 293.4 & 4.04 & 0.33 \\
& 299.9 & 6.54 & 0.38 \\
\hline \multirow{3}{*}{0.05} & 278.9 & 1.25 & 0.21 \\
& 293.9 & 2.13 & 0.12 \\
& 299.7 & 3.46 & 0.28 \\
\hline \multirow{3}{*}{0.11} & 280.0 & 0.81 & 0.06 \\
& 293.9 & 1.69 & 0.32 \\
& 299.7 & 3.40 & 0.49 \\
\hline \multirow{3}{*}{1} & 281.3 & 0.61 & 0.06 \\
& 293.6 & 1.09 & 0.18 \\
& 299.7 & 2.20 & 0.29 \\
\hline \multirow{5}{*}{5} & 279.0 & 0.24 & 0.05 \\
& 293.0 & 0.73 & 0.06 \\
& 299.4 & 1.12 & 0.10 \\
\hline
\end{tabular}

case of BODIPY-C12-HPE at $\sim 300 \mathrm{~K}$, the diffusion coefficient is $\left\langle D_{\tau}\right\rangle=6.54 \cdot 10^{-8} \mathrm{~cm}^{2} / \mathrm{s}$ for POPC hydrated with bidistilled water. When $\mathrm{NaCl}$ concentration increases to $110 \mathrm{mM}$, then the diffusion coefficient decreases to $\left\langle D_{\tau}\right\rangle=3.40 \cdot 10^{-8} \mathrm{~cm}^{2} / \mathrm{s}$ and for $5 \mathrm{M} \mathrm{NaCl}$ to $\left\langle D_{\tau}\right\rangle=1.12 \cdot 10^{-8} \mathrm{~cm}^{2} / \mathrm{s}$ (refer to Table 4.8). Increasing the $\mathrm{NaCl}$ concentration more than $110 \mathrm{mM}$ for both markers produced the same diffusion coefficient (within the one standard deviation).

One can see that diffusion becomes slower not only in the present of salt but also by decreasing the temperature (refer to Figure 4.32 and Figure 4.33).

Cross-correlation profiles performed with TRITC DHPE differ from the crosscorrelation profiles performed with BODIPY-C12-HPE. The reason for more stretched curves may be due to a charge on TRITC DHPE marker. Overall, the time dependence of the correlation curve is slightly more stretched than the fitted profile independent of $\mathrm{NaCl}$ concentrations. Probably, a slight heterogeneity of the diffusion coefficient and the assumption of a Gaussian profile of the focus are sources of this observation (see Section 2.2.1).

The same effect of saturated membranes above given salt concentrations was found by Rappolt using x-ray crystallography [91]. Rappolt investigated POPC, DMPC and DPPC MLVs 
Table 4.9: Mean values of the diffusion coefficient, $\left\langle D_{\tau}\right\rangle$, of TRITC-DHPE in POPC membranes at various temperatures with different $\mathrm{NaCl}$ concentrations recorded in crosscorrelation mode. $\sigma_{\left\langle D_{\tau}\right\rangle}$ is the standard deviation of $\left\langle D_{\tau}\right\rangle$.

\begin{tabular}{|c|c|c|c|}
\hline $\mathrm{C}_{\mathrm{NaCl}}$ & Temp. & $\left\langle D_{\tau}\right\rangle$ & $\sigma_{\left\langle D_{\tau}\right\rangle}$ \\
$\frac{M}{l}$ & $\mathrm{~K}$ & $\cdot 10^{-8} \frac{\mathrm{cm}^{2}}{\mathrm{~s}}$ & $\cdot 10^{-8} \frac{\mathrm{cm}^{2}}{\mathrm{~s}}$ \\
\hline \multirow{3}{*}{0} & 279.6 & 2.69 & 0.12 \\
& 293.5 & 3.40 & 0.35 \\
& 299.5 & 7.75 & 1.19 \\
\hline \multirow{3}{*}{0.01} & 281.6 & 2.39 & 0.39 \\
& 293.0 & 3.94 & 0.29 \\
& 300.8 & 5.52 & 0.69 \\
\hline \multirow{3}{*}{0.05} & 282.2 & 1.65 & 0.17 \\
& 292.7 & 2.52 & 0.39 \\
& 300.8 & 4.52 & 0.32 \\
\hline \multirow{3}{*}{0.08} & 281.0 & 1.56 & 0.16 \\
& 291.9 & 1.89 & 0.22 \\
& 299.6 & 2.84 & 0.28 \\
\hline \multirow{3}{*}{0.11} & 281.3 & 1.24 & 0.43 \\
& 293.2 & 1.83 & 0.14 \\
& 299.2 & 1.71 & 0.52 \\
\hline \multirow{3}{*}{1} & 280.0 & 1.47 & 0.17 \\
& 293.0 & 1.69 & 0.19 \\
& 300.7 & 1.62 & 0.52 \\
\hline \multirow{3}{*}{5} & 278.6 & 0.88 & 0.32 \\
& 293.6 & 1.89 & 0.48 \\
& 299.7 & 1.38 & 0.10 \\
\hline
\end{tabular}

in the presence of monovalent ions: $\mathrm{Li}^{+}, \mathrm{Na}^{+}, \mathrm{K}^{+}$and $\mathrm{Cl}^{-}$. At concentrations above $70 \mathrm{mM}$, a decrease in the area per lipid molecule and an increase in the bilayer thickness was found. This is relevant to a gel ordered phase. The strongest effect of induced phase separation was found for lithium ions with respect to its concentration. However, this effect stays constant above a certain salt concentration. The membrane saturation with salt is an interesting observation since the concentration of $\mathrm{NaCl}$ is $\sim 150 \mathrm{mM}$ in biological cells. 


\subsubsection{Molecular Dynamic simulations}

Molecular Dynamic simulations (MD) were performed by Böckmann in a collaboration with the Theoretical Molecular Biophysics Group of MPI for biophysical Chemistry, lead by Grubmüller. MD simulations are time consuming, but in contrast to the Ising model used for the current MC simulations, they yield molecular details in terms of position and motion of atoms. Vibrations in the atomic structure of molecules are modelled in MD simulation according to Newton first law of motion. This method gives information about the conformation and dynamics of all atoms in the lipid membrane. But, for exact calculations, a large amount of computational time is required. Usually, one calculates with them processes that are occurring on the 1-100 ns time scale. But biological processes take a longer time (e.g. diffusion of the label trough the focus takes ms).

A bilayer system with 128 POPC lipids well hydrated (40 waters molecules per one lipid molecule) was studied. A pre-equilibrated POPC bilayer provided from Tieleman was used as a starting structure [117]. Force field parameters for the lipids were taken from the GROMOS87 forcefield [8]. Electrostatic interactions were calculated with the Particle-Mesh Ewald Method (PME) [20]. Three simulations at $300 \mathrm{~K}$ of more than $100 \mathrm{~ns}$ each were carried out. The first simulation was performed in the absence of salt (i.e. only with water molecules), the second simulation for $50 \pm 30 \mathrm{mM} \mathrm{NaCl}$ and the third simulation for $220 \pm 30 \mathrm{mM} \mathrm{NaCl}$. The diffusion coefficient, $D_{\tau}$, was estimated from the slope of the averaged mean-square displacement of the centre of mass, $x(t)$, of single lipids,

$$
D_{\tau}=\frac{1}{4} \lim _{t \rightarrow \infty} \frac{1}{\tau}\left\langle\left|x\left(t_{0}\right)-x\left(t_{0}+\tau\right)\right|^{2}\right\rangle_{t_{0}} .
$$

In Figure 4.36, displacements for POPC with water and with both $\mathrm{NaCl}$ concentrations as well as a distribution of displacements for pure POPC after 100 ps, $6 \mathrm{~ns}$ and $40 \mathrm{~ns}$ are shown.

Figure 4.38 and Figure 4.37 show the diffusion coefficient derived from the one-component fits of FCS profiles and the results from the MD simulations.

Solid lines represent $D \propto \ln [\mathrm{NaCl}]$ dependence fitted to experimental data. The adsorption of sodium ions affects the electrostatic potential of the membrane. In high-field Gouy-Chapman theory, the electrostatic potential, $\Psi_{0}$, has a logarithmic dependence on a surface charge density, $\sigma$, (for $\sigma>0$ ) and ionic strength, $c$, as given by [73], 


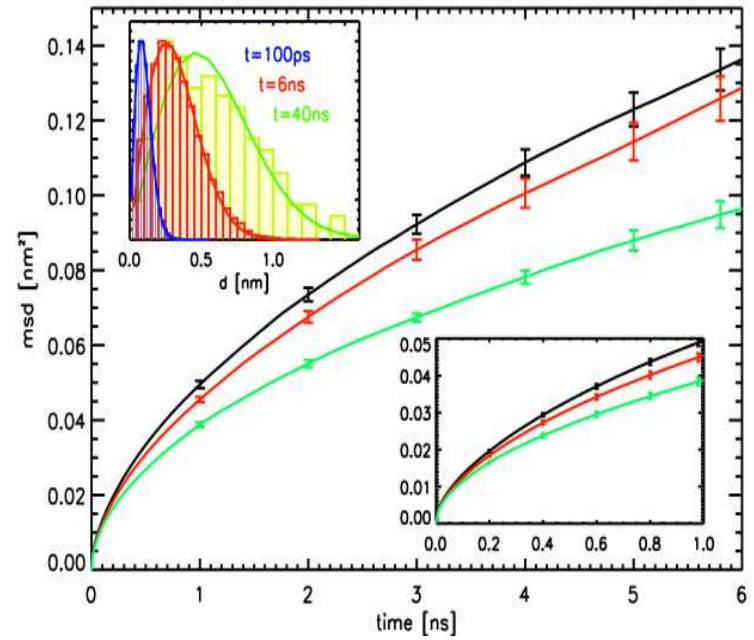

Figure 4.36: Mean-square displacements of POPC lipids computed from the MD simulations without $\mathrm{NaCl}$ (black), at $50 \mathrm{mM} \mathrm{NaCl}$ (red), and at $220 \mathrm{mM} \mathrm{NaCl}$ (green curves). The lower inset shows the same data for $0-1 \mathrm{~ns}$. The distribution of displacements for the simulations without ions, $n(d)$, is given in the upper inset at three different times, $t$. The solid lines display a fit to the solution of the two-dimensional diffusion equation, $n(d) \sim d \cdot \exp \left(-\frac{d^{2}}{4 D_{\tau} t}\right)[11]$.

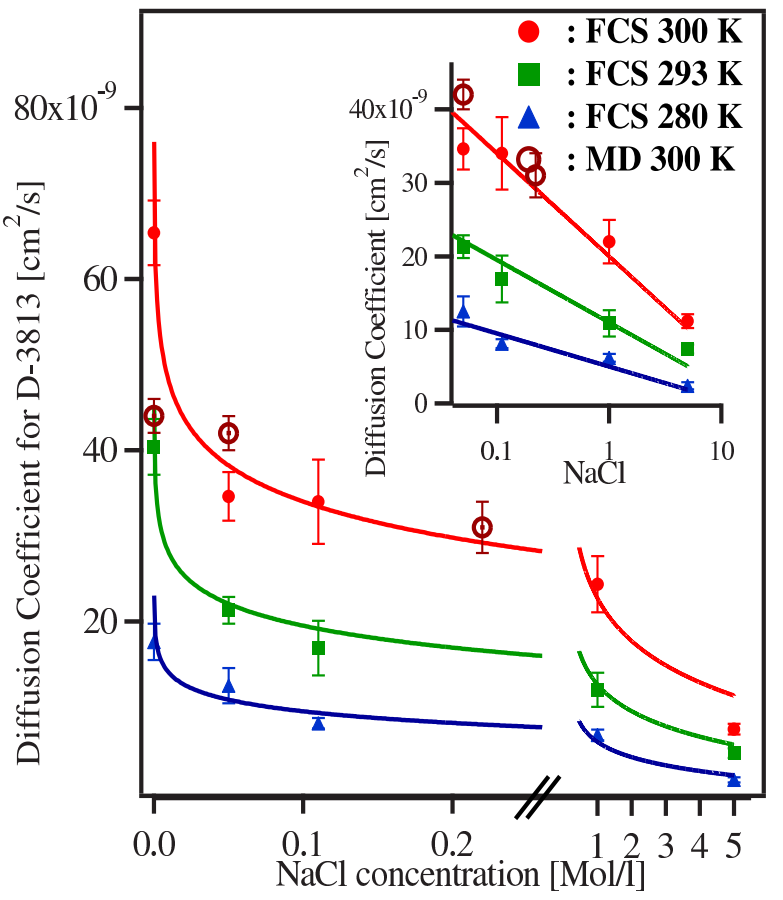

Figure 4.37: Diffusion coefficient of BODIPY-C12-HPE in POPC multilayers as a function of $\mathrm{NaCl}$ concentration and temperature. The filled symbols represents FCS experiments and the open symbols the Molecular Dynamic simulation. Solid lines illustrate the trend only. Semilogarithmic plots (inserts) display a linear behaviour. 


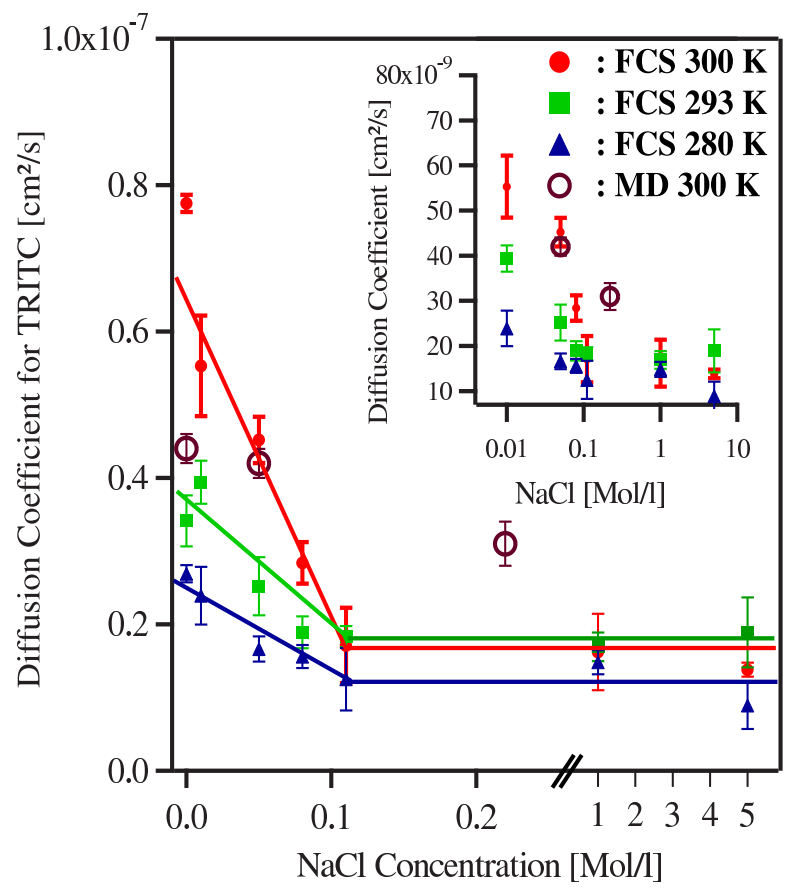

Figure 4.38: Diffusion coefficient of TRITC DHPE in POPC multilayers as a function of $\mathrm{NaCl}$ concentration and temperature. The filled symbols represents FCS experiments and open symbols represent the Molecular Dynamic simulation. Solid lines illustrate the trend only.

$$
\Psi_{0}=\frac{2 k T}{e} \ln \left(\sqrt{\frac{2 \pi \cdot z^{2}}{\epsilon c k_{B} T}} \cdot \sigma\right)
$$

where: $e$ is the charge,

$k_{B}$ is Boltzmann's constant,

$T$ is the absolute temperature,

$z$ is the valence of ions and

$\epsilon$ is the dieelectric constant.

Therefore, both data sets in a semilogarithmic plot are given (see inserts of Figure 4.38 and Figure 4.37).

The values of $D_{\tau}$, its standard deviation , $\sigma_{D_{\tau}}$, coming from MD simulations after $30 \mathrm{~ns}$ of going to equilibrium are shown in Table 4.10 .

Diffusion coefficients obtained from both FCS experiments and MD simulations are in good agreement (compare Table 4.33), with Table 4.32 
Table 4.10: The diffusion coefficient, $D_{\tau}$, and its standard deviation, $\sigma_{D_{\tau}}$, at $300 \mathrm{~K}$ for different salt concentration obtained in Molecular Dynamic simulations. $\frac{A}{L}$ is the computed area per lipid and $\sigma_{\frac{A}{L}}$ is its standard deviation. $a$ is the increase in the distance between the two monolayers.

\begin{tabular}{|c|c|c|c|c|c|}
\hline $\begin{array}{c}\mathrm{C}_{\mathrm{NaCl}} \\
\frac{\mathrm{mM}}{l}\end{array}$ & $\begin{array}{c}D_{\tau} \\
\cdot 10^{-8} \frac{\mathrm{cm}^{2}}{\mathrm{~s}}\end{array}$ & $\begin{array}{c}\sigma_{D_{\tau}} \\
\cdot 10^{-8} \frac{\mathrm{cm}^{2}}{\mathrm{~s}}\end{array}$ & $\begin{array}{c}\frac{A}{L} \\
n m^{2}\end{array}$ & $\begin{array}{c}\sigma_{\frac{A}{L}} \\
\mathrm{~nm}^{2}\end{array}$ & $\begin{array}{c}a \\
\cdot 10^{-10} \mathrm{~m}\end{array}$ \\
\hline 0 & 3.9 & 0.3 & 0.655 & 0.011 & 0 \\
\hline $50 \pm 30$ & 3.7 & 0.4 & 0.625 & 0.011 & 1.1 \\
\hline $220 \pm 30$ & 2.6 & 0.3 & 0.606 & 0.009 & 2.2 \\
\hline
\end{tabular}

The diffusion coefficient, calculated for the first 100 to $500 \mathrm{ps}$ of simulation time, has values between $0.8 \cdot 10^{-7} \mathrm{~cm}^{2} / \mathrm{s}$ and $1.6 \cdot 10^{-7} \mathrm{~cm}^{2} / \mathrm{s}$ which are comparable to $D_{\tau}$, obtained from neutron scattering experiments [117]. However, the self-diffusion coefficients calculated after $30 \mathrm{~ns}$ of going to equilibrium from the $100 \mathrm{~ns}$ simulations $\left(D_{\tau}=2.6 \div 3.9 \cdot 10^{-8} \mathrm{~cm}^{2} / \mathrm{s}\right)$, are consistent with those measured on a millisecond time scale (see Figure 4.37). This suggests that most of the relaxation processes relevant for lipid diffusion are faster than the simulation time scale ( $<100 \mathrm{~ns})$. The values of $D_{\tau}$, calculated according to the extention by Saffman and Delbrück Einstein for Brownian motion which contains the viscosity of the membrane, (Eq. 2.10) are equal to $4.2 \cdot 10^{-8} \mathrm{~cm}^{2} / \mathrm{s}$ for pure POPC and $3.9 \cdot 10^{-8} \mathrm{~cm}^{2} / \mathrm{s}$ in the presence of salt. These values only partially explain the decrease in the self-diffusion coefficient observed in the experiment and in the simulations meaning that the Saffman-Delbrück model is not an adequate approach for diffusion of lipids in membranes. Although the viscosity is a factor affecting diffusion processes in membranes, it only partially describe a lowering of diffusion constant.

The analysis of MD simulated membranes in the presence of salt explains the decrease in the diffusion coefficient when increasing the salt concentration. In MD snapshots (Figure 4.39), one can see that the sodium ions (red spheres) penetrate deeply into the membrane. The molecular dynamic simulations show a (tight) binding of sodium ions to an average of three lipids (see Figure 4.40).

However, this binding forms larger complexes (nanodomains) with reduced mobility. These nanodomains are probably not long-lived enough in FCS experiment. The correlation profiles were well fitted with a one component fit, although there were nanodomains. This suggests that our fitting method is not adequate for analysing microstructures and nanostructures smaller than the focal diameter. 


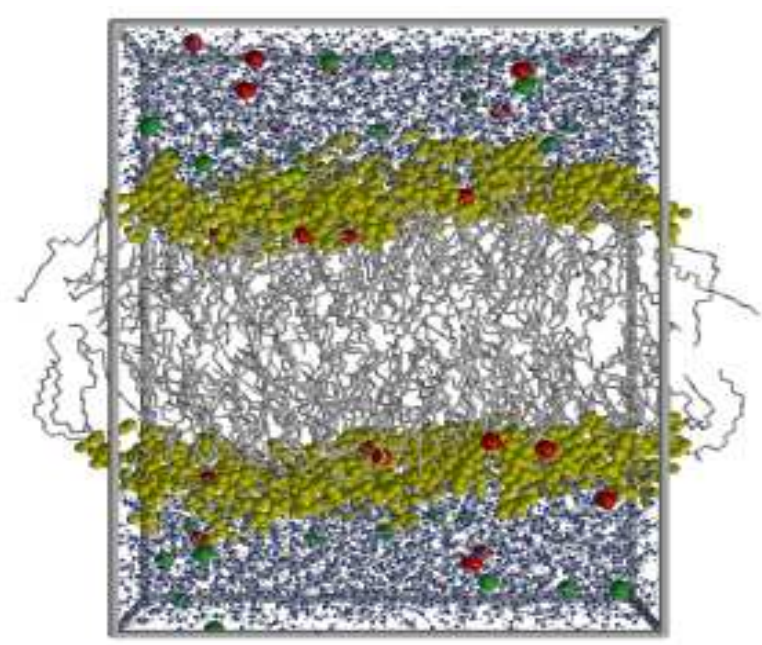

Figure 4.39: Simulation system (128 POPC lipids: grey carbon tails, yellow hydrophilic headgroups, light blue water molecules, red sodium ions, green chlorine ions) [11].

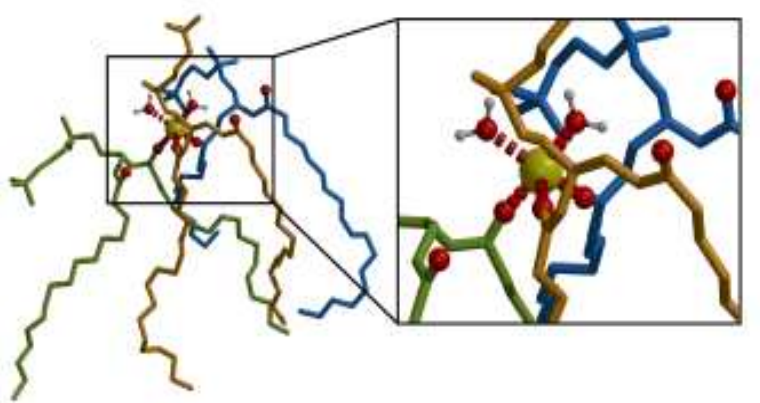

Figure 4.40: Typical coordination of a bound sodium ion (yellow) by three POPC lipids and two water molecules [11].

As a consequence of cluster formation, it was observed that the average area per lipid decreases (see Table 4.i0). The third effect was that the thickness of the bilayer increases by approximately $2 \cdot 10^{-10} \mathrm{~m}$ (refer to Table 4.10). These effects increase the order parameter of the fatty acyl chains. They are characteristic for a gel ordered phase. It was proposed that this deep binding at the interface between the hydrophobic and the hydrophilic region is an explanation for the phase separation in POPC membranes. This was confirmed by x-ray crystallography in that $\mathrm{NaCl}$ concentrations induce phase separation in POPC membranes [9i].

This study showed that ions affect the lateral organisation of artificial membranes and therefore the relevant behaviour in biological membranes. Moreover, salt influences the diffusion processes in membranes so it is important to investigate the influence of salt on the membrane structure if one wants to understand the signal transduction in a neuronal cell. The interpreta- 
tion of calorimetric profiles in biomembranes has been also improved since they are sinked in various salt buffers. The changing chain fluidity of lipids can be important for many biological situations, particularly when transient effects occur in biological membranes. This study also demonstrated the role of monovalent ions in organising the membrane. The role of the membrane as a buffer for ions so far has been underestimated.

This work was published in the Biophysical Journal, 2003 [11]. 


\section{Chapter 5}

\section{Discussion and Conclusions}

The aim of this study was to link domain and microdomain formation to diffusion behaviour in artificial planar membranes using Fluorescence Correlation Spectroscopy and Monte Carlo simulations. The important part of the study was to construct a confocal fluorescence setup which is able to record single molecules, including fast data acquisition with hardware correlators. It consists of a continuous wave 532-nm green Nd:Yag laser, water immersion objective equipment with a nanoposition XYZ-system and two avalanche photodiodes.

For years the investigation of membrane structure and composition has been of a great interest $[6 ; 32 ; 52 ; 55 ; 56 ; 59 ; 72 ; 91 ; 115 ; 122]$. It has been established from experimental [22; $13 ; 36 ; 59 ; 118]$ and theoretical $[56 ; 81 ; 82]$ studies that the membrane structure is not homogenous but contains various heterogenities. The most attractive region in lipid phase diagrams of binary mixtures is that corresponding to the coexistence of gel and fluid domains. Superposition of gel and fluid domains in the phase coexistence region on Giant Unilamellar Vesicles (GUVs) was observed. The direct observation and analysis of gel and fluid domains were possible by Confocal Fluorescence Microscopy (CFM). There, phases can be identified on the known partition behaviour of a given fluorescent marker. With CFM, macroscopic domains can be visualised. Their shapes depend on the lipid composition and on the temperature of the measurement. In the case of DLPC:DPPC mixtures, the gel domains are presented as thin lines on a vesicles surface. In the case of DMPC:DSPC mixtures, the gel domains show a "dendritic shape". Good agreement with the work done by Bagatolli [6; 22] or Korlach [59] was obtained.

The other method which makes it possible to produce an image of domain formation is Atomic Force Microscopy (AFM) [85]. Nielsen at al. was able to detect domains in DMPC 
and DSPC monolayers supported on a mica at their melting transitions. Hollars at al. presents submicron size DPPC domains by using CFM, AFM and Near-Field Scanning Optical Microscopy (NSOM) [48]. However, one should keep in mind that the mica support can interact weakly with lipids and therefore influence domain formation.

There are also indirect methods of mapping domains e.g. Fluorescence Recovery After Photobleaching (FRAP) or Fluorescence Correlation Spectroscopy (FCS). It is possible to distinguish fluid and gel domains by the different values of their translational diffusion coefficient, $D_{\tau}$.

Feigenson, Vaz and Periasamy have investigated diffusion processes by using Fluorescence Recovery after Photobleaching $[1 ; 86 ; 92 ; 108]$. The values of diffusion coefficients obtained with FRAP ranged between $D_{\tau}=10^{-10} \mathrm{~cm}^{2} / \mathrm{s}$ and $10^{-11} \mathrm{~cm}^{2} / \mathrm{s}$ in a gel phase and between $D_{\tau}=10^{-8} \mathrm{~cm}^{2} / \mathrm{s}$ and $10^{-7} \mathrm{~cm}^{2} / \mathrm{s}$ in a fluid phase [70].

Vaz et al. has studied DMPC bilayers in excess water labelled with NBD-DMPE [1]. In a fluid phase, at $307 \mathrm{~K}$, Vaz et al. obtained a diffusion coefficient equal to $6.9 \cdot 10^{-8} \mathrm{~cm}^{2} / \mathrm{s}$, while at $319 \mathrm{~K}$ a value of $11.0 \cdot 10^{-8} \mathrm{~cm}^{2} / \mathrm{s}$ was obtained and at $331 \mathrm{~K}$ a value of 16.2 . $10^{-8} \mathrm{~cm}^{2} / \mathrm{s}$ was obtained. In a gel phase at $283 \mathrm{~K}$, Vaz et al. obtained values smaller than $10^{-10} \mathrm{~cm}^{2} / \mathrm{s}$. For other fluid bilayers like DSPC at $331 \mathrm{~K}$, the diffusion coefficient equal to $13.2 \cdot 10^{-8} \mathrm{~cm}^{2} / \mathrm{s}$ has been found and for DLPC at $293 \mathrm{~K}, D_{\tau} \sim 5 \cdot 10^{-8} \mathrm{~cm}^{2} / \mathrm{s}$.

There is also a difference in $D_{\tau}$ depending on the method used for the measurement. The diffusion coefficient in a fluid membrane derived from FCS and FRAP is slower than that derived from neutron scattering measurements $\left(D_{\tau}=4 \cdot 10^{-6} \mathrm{~cm}^{2} / \mathrm{s}\right.$ and $\left.D_{\tau}=1.6 \cdot 10^{-7} \mathrm{~cm}^{2} / \mathrm{s}\right)$ [87; 112] or excimers technique $\left(D_{\tau}=1.6 \cdot 10^{-7} \mathrm{~cm}^{2} / \mathrm{s}\right.$ for DPPC at $323 \mathrm{~K}$ and $D_{\tau}=0.65$. $10^{-7} \mathrm{~cm}^{2} / \mathrm{s}$ for DMPC at $303 \mathrm{~K}$ ) [34]. The difference in these values is due to the neutron scattering technique and excimers technique observing microscopic diffusion behaviour as explained by the free volume model, whereas FCS or FRAP measures the macroscopic diffusion.

Fluorescence Correlation Spectroscopy (FCS) was utilised as a tool to characterise molecular mobility in different phases. Diffusion processes in artificial lipid membranes containing different amount of gel phase at various temperatures were investigated. The systems consisted of hydrated multilamellar planar membranes (stacks) made from mixtures of various phospholipids with different chain lengths (DLPC:DPPC, DMPC:DSPC) supported on coverslips. 
The confocal setup makes it possible to detect even single molecules (i.e. very diluted label solutions that do not disturb original properties of lipid bilayers could be used). Moreover, according to the free volume theory, diffusion of a fluorescent lipid analogue is almost independent of its chain length and it is only dependent on the temperature [34]. Galla et al. has reported that the values of diffusion constant of PDA in various lipids such as DPPE, DPPA, and DPPC at $343 \mathrm{~K}$ differ at most by $20 \%$. This shows that the diffusion coefficients of diluted dye molecules ( 1 dye per 100 lipids) of similar size and mass to the lipid molecules are comparable to the lipid diffusion coefficients.

With FCS, it was indirectly observed that existence of gel and fluid domains in the phase coexistence region occurs. Fluorescent intensity fluctuations, analysed via a correlation function, gave information on the translational diffusion coefficient, $D_{\tau}$, and the diffusion time, $\tau_{D}$, as well as on the mean number of dye molecules in the laser focus $\langle N\rangle$.

The fluorescent marker moves faster in fluid domains than in gel domains since the fluid phase is loosely packed allowing greater molecular motions. In the more ordered gel phase molecular motions are reduced.

A simply diffusion model in a pure gel and a pure fluid phase was used assuming that there are not any obstacles or heregonities influencing diffusion processes. Therefore the FCS curves were fitted with a one component fit. A fast diffusion coefficient in fluid domains at high temperatures was observed $\left(e . g . D_{\tau}=5.04 \cdot 10^{-8} \mathrm{~cm}^{2} / \mathrm{s}\right.$ for DSPC at $330.4 \mathrm{~K}$ ) in comparison to gel domains at low temperatures (e.g. $D_{\tau}=8.6 \cdot 10^{-10} \mathrm{~cm}^{2} / \mathrm{s}$ for DSPC at $290.6 \mathrm{~K}$ ). The same observation was found for DLPC:DPPC mixtures with a fast diffusion coefficient in fluid domains of DLPC at high temperatures (e.g. $\left.302 \mathrm{~K}, D_{\tau}=6.72 \cdot 10^{-8} \mathrm{~cm}^{2} / \mathrm{s}\right)$ and in gel domains at low temperatures (e.g. $281.7 \mathrm{~K}, D_{\tau}=2.08 \cdot 10^{-9} \mathrm{~cm}^{2} / \mathrm{s}$ ).

The results are consistent with those of Korlach et al. [59] or Scherfeld from the Biophysics group of MPI for biophysical Chemistry led by Prof. Schwille [58].

Korlach investigated GUVs labelled with BODIPY-PC with a 5 mol\% charged POPS as vesicle stabiliser at room temperature. Korlach reported $D_{\tau}$ equal to $3 \cdot 10^{-8} \mathrm{~cm}^{2} / \mathrm{s}$ for pure DLPC, whereas for DLPC:DPPC mixtures (80:20, 50:50, 60:40, 20:80), the diffusion constant of a fast component was equal to 4 to $5 \cdot 10^{-8} \mathrm{~cm}^{2} / \mathrm{s}$ and $D_{\tau}$ for a slow component was equal to $2 \cdot 10^{-10} \mathrm{~cm}^{2} / \mathrm{s}$.

Scherfeld has studied diffusion on GUVs marked with $\mathrm{DiIC}_{18}$ as a fluorescent dye at room temperature. Scherfeld has used a $5 \mathrm{~mol} \%$ solution of charged DPPS in order to stabilise 
vesicles. Scherfeld has observed $D_{\tau}$ on a level of $7 \cdot 10^{-8} \mathrm{~cm}^{2} / \mathrm{s}$ for DLPC. For the mixture, DLPC:DPPC 60:40, and DLPC:DPPC 40:60, the diffusion constant of a fast component was equal to $6 \cdot 10^{-8} \mathrm{~cm}^{2} / \mathrm{s}$ and $D_{\tau}$ of a slow component was equal to $4 \cdot 10^{-10} \mathrm{~cm}^{2} / \mathrm{s}$. For GUVs made of DMPC:DSPC 50:50, $D_{\tau}$ of a fast component was equal to $4 \cdot 10^{-8} \mathrm{~cm}^{2} / \mathrm{s}$ and $D_{\tau}$ of a slow component was equal to $1 \cdot 10^{-10} \mathrm{~cm}^{2} / \mathrm{s}$.

However, a faster diffusion constant in the gel phase in comparison to measurements on GUVs was found in the current study. The difference may be due to the additional undulations on a GUV surface on long time scales, thus resulting in a lower diffusion coefficient as well as from differences in FCS setups.

In this study also diffusion processes in a gel and fluid coexistence phase have been studied. In an intermediate temperature regime, simple diffusion was found to be insufficient to describe the observed correlation curves. Assuming the sum of two diffusing species improved curve fitting. Firstly the diffusion behaviour was explained with the superposition of two diffusion coefficients as a result of the gel and fluid domain coexistence. The following phenomena in a gel and fluid coexistence region was observed: that the diffusion coefficient of a fast component (e.g. $3.34 \cdot 10^{-8} \mathrm{~cm}^{2} / \mathrm{s}$ for DMPC:DSPC $70: 30$ at $309.2 \mathrm{~K}$ ) is a little bit lower in comparison to the diffusion coefficient in pure fluid domains $\left(e . g .5 .17 \cdot 10^{-8} \mathrm{~cm}^{2} / \mathrm{s}\right.$ for DMPC:DSPC 70:30 at 319.2 K). The diffusion coefficient of a slow component (e.g. $2.58 \cdot 10^{-9} \mathrm{~cm}^{2} / \mathrm{s}$ for DMPC:DSPC 70:30 at $309.2 \mathrm{~K}$ ) is a little bit faster in comparison to the diffusion coefficient in pure gel domains (e.g. $1.39 \cdot 10^{-9} \mathrm{~cm}^{2} / \mathrm{s}$ for DMPC:DSPC 70:30 at $289.3 \mathrm{~K}$ ). It was observed that a decrease in temperature results in a lower mean diffusion coefficient. This is due to a relative increase in the gel domain area with respect to the fluid domain. These observation were found for both mixtures, DLPC:DPPC and DMPC:DSPC, respectively, for mainly all molar fractions.

Values of diffusion coefficients were obtained by using the fitting procedure assuming macroscopic gel and fluid phase separation. However, the shape of the cross-correlation profiles in a gel and fluid coexistence region (because of decreasing fast $D_{\tau}$ and increasing slow $D_{\tau}$ ) may predict that there are microdomains, whose length is smaller than the focus diameter (754 nm). It has been hypothesised that a diffusion constant of a lipid which diffuses slowly inside gel microclusters contribute to the fast diffusion constant of a lipid diffusing inside fluid macrodomains and therefore, the observed value of fast $D_{\tau}$ is lower. The fast diffusion constant of a lipid diffusing inside fluid microdomains which are proposed inside gel macrodomains increases the value of slow $D_{\tau}$. The existence of microdomains, which could 
not be resolved by conventional methods as they are below the resolution limit of e.g. CFM, was proposed. On the other hand a change of the fast and slow diffusing component in comparison to the pure phases may be explained due to the gel-fluid fluctuations in the system, which are neglected with the two diffusion component fitting procedure. Also the changed shape of FCS profiles in coexistent phases may be a result of an interplay of microdomains and fluctuations. These hypotheses required additional investigation.

Therefore, the study of membrane thermodynamics with the help of Monte Carlo simulations (MC) based on the two-state Ising model, was employed. In an Ising model, lipids are represented by points on a hexagonal lattice. Every lattice point is occupied by one lipid chain which can exist in either a gel or a fluid state. The MC simulations make use of thermodynamical properties of lipid mixtures, such as heat capacities, melting points and melting entropies derived from calorimetric measurements.

The simulated $c_{p}$-profiles are in quantitative agreement with the measured heat capacity curves over a broad temperature range, thus provide an adequate description of our two component model system. The heat capacity curves for the molar fraction of the DMPC:DSPC mixture from $10 \%$ to $100 \%$ DSPC were reproduced.

This MC model is used in order to describe the main phase transition behaviour of DMPC:DSPC lipid mixtures. Effects which result in a pretransition like they can be clearly seen in one component systems are neglected (see Figure 2.16). The formation of the ripple phase at the given temperature regime was observed experimentally by using AFM [64 and also described theoretically [41]. Passing over the influence of a pretransition and a ripple phase formation should not produce a high deviation in explaining the main transition.

From these simulations, one can deduce matrix snapshots. The MC snapshots showed existence of microscopic gel and fluid inheterogenities in huge fluid and gel macrodomains. On MC snapshots lipids also fluctuate between gel and fluid state. The highest gel-fluid fluctuations have been obtained on the phase limits.

Our experimental correlation profiles can be very well described with theoretical calculations, thus suggesting that the simple two-state Ising model is good enough to study interactions between lipids resulting in micro- and macrodomain formations in membranes. However real lipids are not two states entities and they exist in intermediate states. These ones could be also found in current model through averaging the states of one single lipid [105]. It might be that the fluid or gel microdomains seen in the snapshots are not real gel or real fluid domains, but intermediates in which the highest fluctuations are monitored. 
Subdiffusion in such microdomians with intermediates might explain many interesting biological questions, since it is based on the thermodynamics of the system. Using superposition of two diffusing species restricts the understanding of all of the diffusion processes going on membranes to only a few. Moreover, even some of them can not be analysed since they contribute to one of the two diffusion processes.

MC simulations gave information not only about membrane dynamics (via diffusion), but also about membrane kinetics via the relaxation time [51]. Since the FCS results are well described by the MC simulations, one can analyse the simulations in more detail to arrive at a deeper understanding of the micro-, meso- and nanoscopic ${ }^{1}$ details of the membrane structure.

More recent studies have shown that huge gel and fluid domains are not homogeneous structures as originally predicted. Domains and microdomains differ in size depending on lipid composition and the method of measurement used.

Gliss et al. detected $10 \mathrm{~nm}$ gel microdomains of DAPC in a DMPC:DAPC mixture by AFM and less than $10 \mathrm{~nm}$ gel microdomains of DSPC in a DMPC:DSPC mixture by neutron scattering experiments [36]. Microdomains have been visualised in the gel and the coexistent phase of mixtures.

Ratto et al. has observed 80-140 nm gel domains in DLPC:DPPS supported bilayers by using AFM and FRAP [92]. Ratto et al. found that fluid phase domains decrease in size whereas the gel fraction increases. According to our classification of micro- and macrodomains, Ratto et $a l$. has detected macrodomians (microdomains do not vary in size, see Section 4.2.2.). However, it is difficult to precisely measure the microdomain size since they are close together and begin to aggregate and form clusters. Another important observation found by Ratto et $a l$. with FRAP is that the diffusion coefficient decreases from $8.5 \cdot 10^{-8} \mathrm{~cm}^{2} / \mathrm{s}$ for pure DLPC membranes to $3.49 \cdot 10^{-8} \mathrm{~cm}^{2} / \mathrm{s}$ for a 50:50 DLPC:DSPC mixture and finally to a value of $5 \cdot 10^{-10} \mathrm{~cm}^{2} / \mathrm{s}$ for a 35:65 DLPC:DSPC mixture. For domain diameters larger than $200 \mathrm{~nm}$, the $D_{\tau}$ is no longer size dependent. The lowering of the diffusion constant with increasing gel fraction is consistent with our results.

The microdomains, between $25 \mathrm{~nm}$ and $50 \mathrm{~nm}$, have been found in DLPC:DPPC as well as in DLPC:DPPC:cholesterol mixtures using AFM by Tokumasu [118]. In this study, huge domains were mainly observed only at the edges of membrane whereas microdomains were

\footnotetext{
${ }^{1}$ The distinction between the micro- and nanoscopic domains is as follows: microscopic length scale $\leq 1 \mu \mathrm{m}$ : mesoscopic in the range $\approx 10 \mathrm{~nm}-100 \mathrm{~nm}$; nanoscopic length scale $\leq 10 \mathrm{~nm}$.
} 
homogeneously distributed.

All of these AFM experiments only measure the longer lipid fraction in gel microdomains, however a gel microdomain also contains shorter chains in a gel state. Probably, they are very small and hidden by the presence of taller lipid molecules covering or blocking the AFM tip from detecting the shorter chain domains.

In a related project, the influence of various salt concentrations of $\mathrm{NaCl}$ on lateral diffusion of individual lipids in pure POPC membranes was investigated by Fluorescent Correlation Spectroscopy experiments and Molecular Dynamic simulations (MD). MD Simulations were performed in a collaboration with Böckmann from the Theoretical Molecular Biophysics Group of MPI for biophysical Chemistry, lead by Grubmüller [11].

Increasing sodium chloride concentration was found to decrease the self-diffusion of POPC lipids within the bilayer from $D_{\tau}=6.54 \cdot 10^{-8} \mathrm{~cm}^{2} / \mathrm{s}$ to $D_{\tau}=1.12 \cdot 10^{-8} \mathrm{~cm}^{2} / \mathrm{s}$ by $5 \mathrm{M}$ $\mathrm{NaCl}$ at $300 \mathrm{~K}$.

The values of the diffusion coefficient are in good agreement with those found by Vaz et al. [18]. Using FRAP, Vaz et al. has found a diffusion constant of $D_{\tau}=6.1 \cdot 10^{-8} \mathrm{~cm}^{2} / \mathrm{s}$ at $303 \mathrm{~K}$ for POPC membranes marked with NBD-POPE.

The MD simulations showed a tight binding of sodium ions to an average of three lipids. It is assumed that the resulting formation of nanodomains explains the reduced mobility. Even though ion-lipid clusters exist correlation profiles can be well fitted with a one component fit which is proposed for describing a simply diffusion of similar species. It may indicate that microstructures much smaller than focal diameter cannot be seen using this fitting procedure. This makes it necessary to revise the fitting procedure.

This study shows that salt influences diffusion processes in membranes, therefore it is important to investigate this influence for better understanding the signal transudation in $e$.g. a neuronal cell.

Further investigation of domain and microdomain formation via FCS experiments and MC simulations is required. Since there is a good correlation between the measured FCS curve and the calculated curve using Monte Carlo simulations, this method can be used in the future to look at more complex systems such as biological membranes. Biological membranes are composed of a large variety of lipids and proteins and are supported by a cytoskeleton. The 
simple two-component artificial membranes are excellent models for studying micro- and macrodomain formation and provide physical insights relevant to native biological systems

This study is an important step in understanding the micro-, meso- and nanoscopic details of the membrane structure. All of these heterogenities may play an important role in investigating the physiological function of membranes since they are involved in biological processes such as membrane fusion, signal transduction, and virus release at the cell membrane [118]. 


\section{Chapter 6}

\section{Outlook}

The experimental and theoretical studies try to describe the structure and dynamics of a membrane. The dynamics include diffusion and rotation processes of lipids and proteins in membranes. The lipid rotation covers nanoseconds time scales [39; 54; 79], whereas the diffusion spans milliseconds time scales [1].

FCS can be used for studying rotation processes [3; 26; 54]. The polarised light predominantly excites fluorophores of a certain dipole orientation (photoselection) which than emit polarised light. The orientation of the emitting dipoles can be changed due to rotation. Therefore the polarisation of the emitted light reflects the rotational movements. Including the polarising beam splitter into the optical detection path, and recording the parallel and perpendicular polarised fluorescence light with two detectors (e.g. APDs) allows to study molecule anisotropy.

In this work, the excitation light was additionally rotated by means of a half wave retarder (LINOS). A $\lambda / 2$ plate introduces a phase shift (retardation) of $180^{\circ}$ between two orthogonal polarisations. If its crystal axis is oriented at an angle $\alpha$ with respect to the polarisation of linear polarised incident light, it will lead to linear polarised light leaving the $\lambda / 2$ plate with the polarisation rotated by $2 \alpha$ (see Figure 6.1).

It is therefore proposed to use a half wave retarder for further anisotropy measurements on membranes. The future analysis of parallel and perpendicular polarised light may provide information about lipid rotation as in [37; 113]. Changing the incident polarisation in the anisotropy experiments allows to account for non-isotropic dipole orientation in the sample (encountered in labelled membranes) as well as for a calibration of the individual detector sensitivity (g-factor). The visualisation of rotation mobility was studied in single molecule anisotropy imaging [39; 102]. In this method the sequence of signals are simultaneously re- 


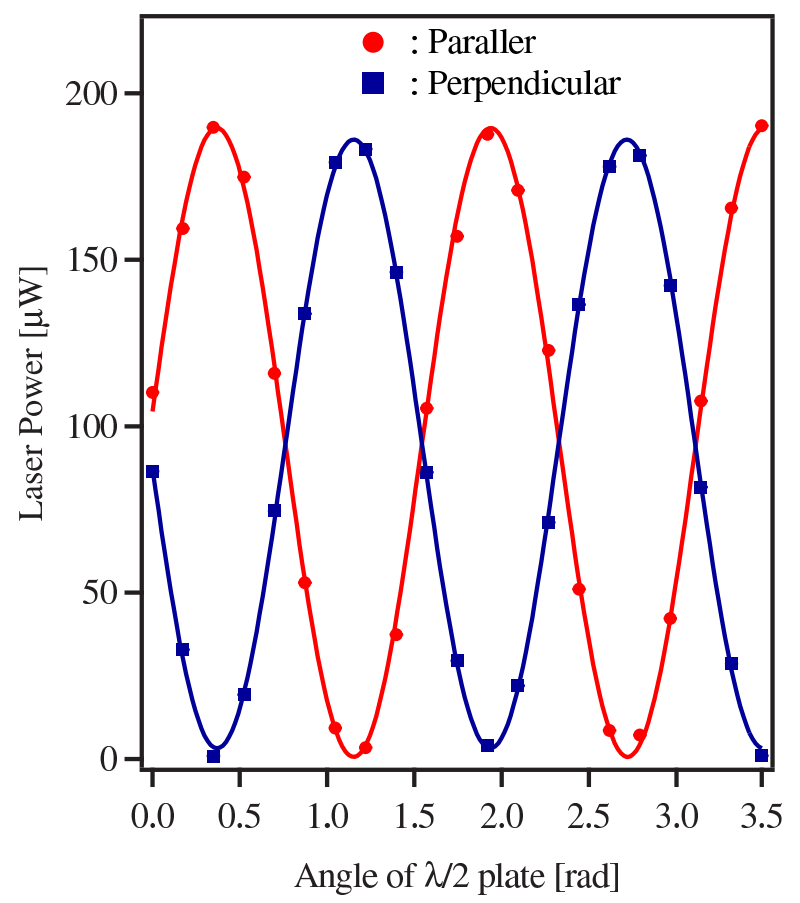

Figure 6.1: The power of the laser (for OD filter 1) measured behind a polarising filter oriented parallel and perpendicular to the laser polarisation as a function of the $\lambda / 2$ plate orientation.

ported on parallel and perpendicular channel with a CCD camera and further investigated. Harms et al. and Moser et al. showed that lipids in a gel state rotate slower as lipids in a fluid state [39; 79]. The rotational mobility of a molecule is highly sensitive to the phase state of the surrounding membrane, therefore its analysis yields information about membrane heterogenities.

Well defined artificial membranes provide physical and chemical insights relevant to native biological systems. In biological systems, diffusion is much more complex in addition to lipid diffusion protein diffusion is also present. Lipids and proteins may form various kinds of heterogenities e.g. rafts which affect diffusion processes. In the near future the diffusion in the cell membrane will be studied by FCS in cooperation with Kirchhoff from the Institute for Botanic of the University of Münster. Since FCS is a minimally invasive method it has been considered to be a very valuable and extremely useful technique for both in vitro and in vivo applications [104]. 


\section{Abbreviations}

AFM: Atomic Force Microscopy,

CFM: Confocal Fluorescence Microscopy,

DSC: Differential Scanning Calorimetry,

FCS: Fluorescence Correlation Spectroscopy,

FRAP: Fluorescence Recovery After Photobleaching,

MC: Monte Carlo simulations,

MD: Molecular Dynamic simulations,

SM: Single Molecule,

MLVs: Multilamellar Vesicles,

GUVs: Giant Unilamellar Vesicles,

DLPC: 12:0-12:0 1,2-dilauroyl-sn-glycero-3-phosphocholine

DMPC: 14:0-14:0 1,2-dimyristoyl-sn-Glycero-3-phosphocholine,

DPPC: 16:0-16:0 1,2-dipalmitoyl-sn-glycero-3-phosphocholine,

DSPC: 18:0-18:0 1,2-distearoyl-sn-glycero-3-phosphocholine,

POPC: 16:0-18:1 PC, 1-palmitoyl-2-oleoyl-sn-glycero-3-phosphocholine,

DiIC16: 1,1'-dihexadecyl-3,3,3',3'- tetramethylindocarbocyanine perchlorate,

DiIC18: 1,1'-dioctadecyl-3,3,3',3'- tetramethylindocarbocyanine perchlorate, 
$\beta$-BODIPY(r) C12-HPE: 2-(4,4-difluoro-5,7-diphenyl-4-bora-3a, 4a-diaza-s-indacene-3-dodecanoyl)1-hexadecanoyl-sn-glycero-3-phosphoethanolamine,

TRITC DHPE: N-(6- tetramethylrhodaminethiocarbamoyl)-1,2-dihexadecanoyl-sn-glycero3-phosphoethanolamine, triethylammonium salt,

TFE: 2,2,2 - Trifluoroethanol,

APD: Avalanche Photodiode,

IC: Internal Conversion,

ISC: InterSystem Crossing,

OD: Optical Density filter 


\section{Bibliography}

[1] Almeida P., Vaz W. Lateral Diffusion in Membranes. In R. Lipowski, E. Sackmann, eds., Structure and Dynamics of Membranes: From Cells to Membranes, Handbook of Biological Physics, pp. 305-357. Elsevier, Amsterdam, 1995.

[2] de Almeida R.F.M., Fedorov A., Prieto M. Spingomyelin/Phospatidylcholine/Cholesterol Phase Diagram: Boundaries and Composition of Lipid Rafts. Biophys. J., 85, pp. 2406-2416, 2003.

[3] Aragón S.R., Pecora R. Fluorescence Correlation Spectroscopy as a probe of molecular dynamics. J. Comput. Phys., 64, pp. 1791-1803, 1976.

[4] Bach D. Calorimetric Studies of Model and Natural Biomembranes. In D. Chapman, ed., Topics in Molecular and structural Biology: Biomembrane Structure and Function, pp. 1-41. Verlag Chemie, Weinheim, 1984.

[5] Bagatolli L.A., Graton E. Two-Photon Fluorescence Microscopy Observation of Shape Changes at the Phase Transition in Phospholipid Giant Unilamellar vesicles. Biophys. J., 77, pp. 2090-2101, 1999.

[6] Bagatolli L.A., Graton E. A Correlation Between Lipid Domain Shape and Binary Phospholipid Mixture Composition in Free Standing Bilayers: A Two-Photon Fluorescence Microscopy Study. Biophys. J., 79, pp. 437-447, 2000.

[7] Bagatolli L.A., Graton E., Khan T.K., Chong P.L.G. A Two-Photon Fluorescence Microscopy Studies of Bipolar Tetraether Giant Liposomes from Thermoacidophilic Archaebacteria Sulfolobus acidocaldarius. Biophys. J., 79, pp. 416-425, 2000.

[8] Berger O., Edholm O., Jähnig F. Molecular Dynamics Simulations of a Fluid Bilayer of Dipalmitoylphosphatidylcholine at Full Hydration, Constant Pressure, and Constant Temperature. Biophys. J., 72, pp. 2002-2013, 1997. 
[9] Biltonen R.L. A Statistical-Thermodynamic View of Cooperative Structural Changes in Phospholipid Bilayer Membranes: Their Potential Role in Biological Function. $J$. Chem. Thermodynamics, 22, pp. 1-19, 1990.

[10] Bloom M., Smith I.C.P. Manifestations of Lipid-Protein Interactions in Deuterium NMR. In A. Watts, J.J.H.H.M.D. Pont, eds., Progress in Protein-Lipid Interactions, pp. 61-88. Elsevier, Amsterdam, 1985.

[11] Böckmann R.A., Hac A., Heimburg T., Grubmüller H. Effect of Sodium Chloride on a Lipid Bilayer. Biophys. J., 85, pp. 1647-1655, 2003.

[12] Boyer D., Tamarat P., Maali A., Lounis B., Orrit M. Photothermal Imaging of Nanometer-Sized Metal Particles Among Scatteres. Science, 297, pp. 1160-1163, 2002.

[13] Brown D.A., London E. Structure and Origin of Ordered Lipid Domains in Biologocal Membranes. J. Membr. Biol., 164, pp. 103-114, 1998.

[14] Caillé A., Pink D.A., de Verteuil F., Zuckermann M.J. Theoretical models for quasitwo-dimensional mesomorpic monolayers and membrane bilayers.

[15] Calvert J.G., Pitts J.N.J. Photochemistry. Wiley, New York, 1989.

[16] Chapman D. Lipid Phase Transitions. In M. Shinitzky, ed., Biomembranes. Physical Aspects, pp. 1-28. VCH, Weinheim, 1993.

[17] Chapman D., Urbina J. Biomembrane Phase Transitions. Studies of Lipid-Water Systems Using Differential Scanning Calorimetry. J. Biol. Chem., 249, pp. 2512-2521, 1974.

[18] Clegg R.M., Vaz W.L.C. Translational Diffusion of Proteins and Lipids in Artificial Lipid Bilayer Membranes. A Comparison of Experiment with Theory. In A. Watts, J.J.H.H.M.D. Pont, eds., Progress in Protein-Lipid Interactions, pp. 173-230. Elsevier, Amsterdam, 1985.

[19] Cohen M., Turnbull D. Molecular Transport in Liquids and Glasses. J. Chem. Phys., 31, pp. 1164-1169, 1959.

[20] Darden T., York D., Pedersen L. Particle Mesh Ewald-an Nlog(N) Method for Ewald Sums in Large Systems. J. Chem. Phys., 98, pp. 10089-10092, 1993. 
[21] Davies M. Infra-Red Spectroscopy and Molecular Structure. Elsevier, New York, 1963.

[22] Dietrich C., Bagatolli L.A., Volovyk Z.N., Thompson N.L., Levi M., Jacobson K., Graton E. Lipids Rafts Reconstructed in Model Mmebranes. Biophys. J., 80, pp. 1417-1428, 2001.

[23] Doniach S. Thermodynamic Fluctuations in Phospholipid Bilayers. J. Phys. Chem., 68, pp. 4912-4916, 1978.

[24] Eggeling C. Analyse von photochemischer Kinetik und Moleküldynamik durch mehrdimensionale Einzelmolekül-Fluoreszenzspektroskopie. Ph.D. thesis, University of Göttingen, 1999.

[25] Eggeling C., Widengren J., Rigler R., Seidel C. Photobleaching of Fluorescent Dyes under Conditions Used for Single-Molecule Detection: Evidence of Two-Step Photolysis. Anal. Chem., 70, pp. 2651-2659, 1998.

[26] Ehrenberg M., Rigler R. Rotational Brownian Motion and Fluorescence Intensity Fluctuations. Chem. Phys., 4, pp. 390-401, 1974.

[27] Eigen M., Rigler R. Sorting Single Molecules: Application to Diagnostics and Evolutionary Biotechnology. Proc. Natl. Acad. Sci. USA, 91, pp. 5740-5747, 1994.

[28] Einstein A. Über die von der molekularkinetischen Theorie der Wärme gefordete Bewegung von in ruhenden Flüssigkeiten suspendieren Teilchen. Ann. Phys. (Leipzig), 17, pp. 548-560, 1905.

[29] Einstein A. Zur Theorie der Brownschen Bewegung. Ann. Phys. (Leipzig), 19, pp. 370-381, 1906.

[30] Elson E.L., Magde D. Fluorecsence Correlation Spectroscopy. I. Conceptual Basis and Theory. Biopolymers, 13, pp. 1-27, 1974.

[31] Fattal D.R., Ben-Shaul A. Lipid Chain Packing and Lipid-Protein Interaction in Membranes. Physica A, 220, pp. 192-216, 1995.

[32] Finean J.B., Michell R.H. Isolation, Composition and General Structure of Membranes. In A. Neuberger, L.L.M. van Deenen, eds., New Comprehensive Biochemistry, vol. 1: Membrane Structure, pp. 1-36. Elsevier/North-Holland Biomedical Press, Amsterdam, 1981. 
[33] Fries J.R., Brand L., Eggeling C., Kölner M., Seidel C.A.M. Quantitative Identification of Diffrent Single Molecules by Selective Time-Resolved Confocal Fluorescence Spectroscopy. J. Phys. Chem., 102, pp. 6601-6613, 1998.

[34] Galla H., Hartmann W., Theilen U., Sackmann E. On Two-Dimensional Passive Randomwalk in Lipid Bilayers and fluid Pathways in Biomembranes. J. Membr. Biol., 48, pp. 215-236, 1979.

[35] Gennis R.B. Biomembranes. Molecular structure and function. Springer, New York, 1989.

[36] Gliss C., Clausen-Schaumann H., Günther R., Odenbach S., Randl O., Bayerl T.M. Direct Detection of Domains in Phospholipid Bilayers by Grazing Incidence Diffraction of Neutrons and Atomic Force Microscopy. Biophys. J., 74, pp. 2443-2450, 1998.

[37] Ha T., Glass J., Enderle T., Chemla D.S., Weiss S. Hindered Rotational Diffusion and Rotational Jumps of Single Molecules. Phys. Rev. Lett., 80, pp. 2093-2096, 1998.

[38] Halstenberg S., Heimburg T., Hianik T., Kaatze U., Krivanek R. Cholesterol-Induced Variations in the Volume and Enthalpy Fluctuations of Lipid Bilayers. Biophys. J., 75, pp. 264-271, 1998.

[39] Harms G.S., Sonnleitner M., Schütz G.J., Gruber H.J., Schmidt T. Single Molecule Anisotropy Imaging. Biophys. J., 77, pp. 2864-2870, 1999.

[40] Heimburg T. Mechanical Aspects of Membrane Thermodynamics. Estimation of the Mechanical Properties of Lipid Membranes Close to the Chain Melting Transition from Calorimetry. Biochim. Biophys. Acta, 1415, pp. 147-162, 1998.

[41] Heimburg T. A Model for the Lipid Pretransition: Coupling of Ripple Formation with the Chain-Melting Transition. Biophys. J., 78, pp. 1154-1165, 2000.

[42] Heimburg T. Monte Carlo Simulations of Lipid Bilayers and Lipid protein Interactions in the Light of Recent Experiments. Current Opinion in Colloid and Interface Science, 5, pp. 224-231, 2000.

[43] Heimburg T. Coupling of Chain Melting and Bilayer Structure: Domains, Rafts, Elasticity and Fusion. In H.T. Tien, A. Ottova-Leitmannova, eds., Planar Lipid Bilayer (BLMs) and Their Applications, pp. 269-293. Elsevier, Amsterdam, 2003. 
[44] Heimburg T., Biltonen R.L. A Monte Carlo Simulation Study of Protein-Induced Heat Capacity Changes and Lipid-Induced Protein Clustering. Biophys. J., 70, pp. 84-96, 1996.

[45] Heimburg T., Marsh D. Thermodynamics of the interaction of proteins with lipid membranes. In K.M. Merz, B. Roux, eds., Biological Membranes: A Molecular Perspective from Computation and Experiment, pp. 405-462. Birkhäuser, Boston, 1996.

[46] Hess S.T., Shaohui H., Heikal A.A., Webb W.W. Biological and Chemical Applications of Fluorescence Correlation Spectroscopy: A Review. Biochemistry, 41, pp. 697-706, 2002.

[47] Hess S.T., Webb W.W. Focal Volume Optics and Experimental Artifacts in Confocal Fluorescence Spectroscopy. Biophys. J., 83, pp. 2300-2317, 2002.

[48] Hollars C.W., Dunn R.C. Submicron Structure in L-alphaDipalmitoylphosphatidylcholine Monolayers and Bilayers Probed with Confocal, Atomic Force, and Near-Field Microscopy. Biophys. J., 75, pp. 342-353, 1998.

[49] Ipsen J.H., Jörgensen K., Mouritsen O.G. Density Fluctuations in Saturated Phospholipid Bilayers Increase as the Acyl-Chain Length Decreases. Biophys. J., 58, pp. 1099-1107, 1990.

[50] Ising E. Beitrag zur Theorie des Ferromagnetismus. Z. Phys., 31, pp. 253-258, 1925.

[51] Ivanova V.P. Theoretical and experimental study of protein-lipid interactions. Ph.D. thesis, University of Göttingen, 2000. The thesis can be downloaded from the web-site of the corresponding author.

[52] Jacobson K., Sheets E.D., Simson R. Revisiting the Fluid Mosaic Model of Membranes. Science, 268, pp. 1441-1442, 1995.

[53] Jähnig F. Molecular Theory of Lipid Membrane Order. J. Phys. Chem., 70, pp. 3279_ 3290, 1979.

[54] Jähnig F. Structural Order of Lipids and Proteins in Membranes: Evaluation of Fluorescence Anisotropy Data. Proc. Natl. Acad. Sci. USA, 76, pp. 6361-6365, 1979.

[55] Jain M.K. Introduction to Biological Membranes. Wiley, New York, 1988. 
[56] Jain M.K., White H.B. Long-Range Order in Biomembranes. Adv. Lipid Res., 15, pp. $1-60,1977$.

[57] Jörgensen K., Klinger A., Braiman M., Biltonen R.L. Slow Nonequilibrium Dynamical Rearrangement of the Lateral Structure of a Lipid Membrane. J. Phys. Chem., 100.

[58] Kahya N., Scherfeld D., Bacia D., Poolman K., Schwille P. Probing Lipid Mobility of Raft-Exibiting Model Membranes by Fluorescence Correlation Spectroscopy. J. Biol. Chem., 278, pp. 28109-28115, 2003.

[59] Korlach J., Schwille P., Webb W.W., Feignson G.W. Characterization of Lipid Bilayer Phases by Confocal Microscopy and Fluorescence Correlation Spectroscopy. Proc. Natl. Acad. Sci. USA, 96, pp. 8461-8466, 1999.

[60] Kuśba J., Li L., Gryczynski I., Piszczek G., Johnson M., Lakowicz J.R. Lateral Diffusion Coefficients in Membranes Measured by Resonance Energy Transfer and a New Algorithm for Diffusion in Two Dimensions. Biophys. J., 82, pp. 1358-1372, 2002.

[61] Lakowicz J.R. Topics in Fluorescence Spectroscopy. Volume 1: Techniques. Plenum Press, New York, 1991.

[62] Lakowicz J.R. Topics in Fluorescence Spectroscopy. Volume 2: Principles. Plenum Press, New York, 1991.

[63] Lee A.G. Lipid Phase Transition and Phase Diagram. Biochim. Biophys. Acta, 472, pp. 285-344, 1977.

[64] Leidy C., Kaasgaarad T., Crowe J.H., Mouritsen O.G., Jörgensen K. Ripples and the Formation of Anisotropic Lipid Domains: Imaging Two- Component Supported Double Bilayers by Atomic Force Microscopy. Biophys. J., 83, pp. 2625-2633, 2002.

[65] Lichtenberg D. Micelles and Liposomes. In M. Shinitzky, ed., Biomembranes. Physical Aspects, pp. 63-95. VCH, Weinheim, 1993.

[66] MacCarthy J., Kozak J. Lateral Diffusion in Fluid Systems. J. Chem. Phys., 77, pp. 2214-2216, 1982.

[67] Magde D., Elson E.L., Webb W.W. Thermodynamic Fluctuations in a Reacting System-Measurement by Fluorecsence Correlation Spectroscopy. Phys. Rev. Lett., 29, pp. 705-708, 1972. 
[68] Makowski L., Li J. X-ray Diffraction and Electron Microscope Studies of the Molecular Structure of Biological Membranes. In D. Chapman, ed., Topics in Molecular and structural Biology: Biomembrane Structure and Function, pp. 43-166. Verlag Chemie, Weinheim, 1984.

[69] Marćelja S. Chain ordering in liquid crystals. I. Even-odd effect. J. Chem. Phys., 60, pp. 3599-3604, 1974.

[70] Marsch D. CRC Handbook of Lipid Bilayers. CRC Press, Boca Raton, Florida, 1990.

[71] Marsh D. ESR Spin Label Studies of Lipid-Protein Interactions. In A. Watts, J.J.H.H.M.D. Pont, eds., Progress in Protein-Lipid Interactions, pp. 143-172. Elsevier, Amsterdam, 1985.

[72] Marsh D. Bilayers, Monolayers, Multilayers and Non-Lamellar Lipid Phases. In M. Shinitzky, ed., Biomembranes. Physical Aspects, pp. 1-28. VCH, Weinheim, 1993.

[73] McLaughlin S. The Electrostatic Properties of Membranes. Ann. Rev. Biophys. Biophys. Chem., 18, pp. 113-136, 1989.

[74] Meseth U., Wohland T., Rigler R., Vogel H. Resolution of Fluorescence Correlation Measurements. Biophys. J., 76, pp. 1619-1631, 1999.

[75] Metropolis N., Rosenbluth A.W., Rosenbluth M.N., Teller A.H., Teller E. Equation of the state calculations by fast comuting machines. J. Chem. Phys., 21, pp. 1087-1092, 1953.

[76] Michonova-Alexova E.I., Sugár I.P. Component and State Separation in DMPC/DSPC Lipid Bilayers: A Monte Carlo Simulation Study. Biophys. J., 83, pp. 1820-1833, 2002.

[77] Milon S., Hovius R., Vogel H., Wohland T. Factors Influencing Fluorecsence Correlation Spectroscopy Measurements on Membranes: Simulations and Experiments. Chem. Phys., 288, pp. 171-186, 2003.

[78] Moerner W.E., Orrit M. Illuminating Single Molecules in Condensed Matter. Science, 283, pp. 1670-1676, 1999.

[79] Moser M., Marsh D., Meier P., Wassmer K.H., Kothe G. Chain Configuration and Flexibility Gradient in Phospholipid membranes. Comparison Between Spin-Label 
Electron Spin Resonance and deuteron Nuclear Magnetic Resonance, and Identification of New Conformations. Biophys. J., 55, 1989.

[80] Mouritsen O.G. Computer Simulation of cooperative phenomena in lipid membranes. In R. Brasseur, ed., Molecular description of biological membrane components by computer aided conformational analysis, pp. 3-83. CRC Press, Boca Raton, 1990.

[81] Mouritsen O.G. Theoretical Models of Phospholipid Phase Transitions. Chem. Phys. Lett., 57, pp. 179-194, 1991.

[82] Mouritsen O.G., Bloom M. Theoretical Models of Phospholipid Phase Transitions. Biophys. J., 46, pp. 141-153, 1984.

[83] Mouritsen O.G., Sperotto M.M. Thermodynamics of Lipid-Protein Interactions in Lipid Membranes: The Hydrophobic Matching Condition. In M. Jackson, ed., Thermodynamics of Cell Surface Receptors, pp. 127-181. CRC Press, Boca Raton, 1992.

[84] Nagle J.F. Theory of the Main Lipid Bilayer Phase Transition. Ann. Rev. Phys. Chem., 31, pp. 157-195, 1980.

[85] Nielsen L.K., Björnholm T., Mouritsen O.G. Fluctuations Caught in the Act. Nature, 404.

[86] Periasamy N., Verkman A.S. Analysis of Fluorophore Diffusion by Continuous Distributions of Diffusion Coefficients: Application to Photobleaching Measurements of Multicomponent and Anomalous Diffusion. Biophys. J., 75, pp. 557-567, 1998.

[87] Pfeiffer W., Schlossbauer G., Knoll W., Farago B., Steyer A., Sackmann E. Ultracold Neutron Scattering Study of Local Lipid Mobility in Bilayer Membranes. J. Phys.I France, 49, pp. 1077-1082, 1988.

[88] Pink D.A., Chapman D. Protein-lipid interactions in bilayer membranes: A lattice model. Proc. Natl. Acad. Sci. USA, 76, pp. 1542-1546, 1979.

[89] Pink D.A., Green T.J., Chapman D. Raman Scattering in Bilayers of Saturated Phosphatidylcholines. Biochem., 19, pp. 349-356, 1980.

[90] Plotnikov V.V., J. M. Brandts L.N.L., Brandts J.F. A New Ultrasensitive Scanning Calorimeter. Analyt. Biochem., 250, pp. 237-244, 1997. 
[91] Rappolt M., Pabst G., Amenitsch H., Laggner P. Salt-induced Phase Separation in the Liquid Crystaline Phase of Phosphatidylcholines. Coll. Surf. A, 183, pp. 171-181, 2001.

[92] Ratto T.V., Longo M.L. Obstructed Diffusion in Phase-Separated Supported Lipid Bilayers: A Combined Atomic Force Microscopy and Fluorescence Recovery after Photobleaching Approach. Biophys. J., 83, pp. 3380-3392, 2002.

[93] Rigler R., Mets U., Widengren J., Kask P. Fluorescence Correlation Spectriscopy with High Count Rate and Low Background: Analysis of Translational Diffusion. Europ. Biophys. J., 22, pp. 169-175, 1993.

[94] Saffman P., Delbrück M. Brownian Motion in Biological Membranes. Proc. Natl. Acad. Sci. USA, 72, pp. 3111-3113, 1975.

[95] Salton M.R.J. Cell Membranes: Their Structure and Functions. In J.R. Villanueva, F. Ponz, eds., Membranes. Structure and Function, pp. 3-7. Academic Press, London, 1970.

[96] Saxton M.J. Lateral Diffusion in an Archipelago. The Effect of Mobile Obstacles. Biophys. J., 52, pp. 989-997, 1987.

[97] Saxton M.J. Single-Particle Tracking: Effect of Corrals. Biophys. J., 69, pp. 389-398, 1993.

[98] Schaffer J., Volkmer A., Eggeling C., Subramaniam V., Striker G., Seidel C.A.M. Identification of Single Molecules in Aqueous Solution by Time-Resolved Fluorescence Anisotropy. J. Phys. Chem., 103, pp. 331-336, 1999.

[99] Schlötzer T. Domänen in Lipidmembranen: Untersuchungen zum Phasenverhalten von binären Lipidmischungen mit Kalorimetrie und konfokaler Fluoreszenmikroskopie. Master's thesis, University of Göttingen, 2002. Masters Thesis.

[100] Schmidt T., Schütz G.J., Baumgartner W., Gruber H.J., Schindler H. Characterization of Photophysics and Mobility of Single Molecules in a Fluid Lipid Membrane. J. Phys. Chem., 99, pp. 17662-17668, 1995.

[101] Schneider M.F., Marsh D., Jahn W., Kloesgen B., Heimburg T. Network Formation of Lipid Membranes: Triggering Structural Transitions by Chain Melting. Proc. Natl. Acad. Sci. USA, 96, pp. 1-6, 1999. 
[102] Schütz G.J., Schindler H., Schmidt T. Imaging Single-Molecule Dichroism. Optics Letters, 22, pp. 651-653, 1997.

[103] Schütz G.J., Schindler H., Schmidt T. Single-Molecule Microscopy on Model Membranes Reveals Anomalous Diffusion. Biophys. J., 73, pp. 1073-1080, 1997.

[104] Schwille P., Korlach J., Webb W.W. Fluorescence Correlation Spectroscopy With Single-Molecule Sensitivity on Cell and Model Membranes. Cytometry, 36, pp. 176$182,1999$.

[105] Seeger H. Theoretische und experimentelle Beschreibung von Diffusionprozessen in einem zweikomponentigen Lipidsystem. Master's thesis, University of Göttingen, 2002. Masters Thesis.

[106] Simons K., Ikonen E. Functional Rafts in Cell Membranes. Nature, 387, pp. 569-572, 1997.

[107] Singer S.J., Nicolson G.L. The Fluid Mosaic Model of Cell Membranes. Science, 175, pp. 720-731, 1972.

[108] Spink C.H., Yeager M.D., Feigenson G.W. Partitioning Behavior of Indcarbocyanine Probes BetweenCoexistin Gel and Fluid Phases in Model Membranes. Biochim. Biophys. Acta, 1023, pp. 25-33, 1990.

[109] Sugár I.P., Biltonen R.L., Mitchard N. Monte Carlo Simulation of Membranes: Phase Transition of Small Unilamellar Dipalmitoylphosphatidylcholine Vesicles. Methods Enzymol., 240, pp. 569-593, 1994.

[110] Sugár I.P., Michonova-Alexova E., Chong P.L.G. Geometrical Properties of Gel and Fluid Clusters in DMPC/DSPC Bilayers: Monte Carlo Simulation Approach using a Two-State Model. Biophys. J., 81, pp. 2425-2441, 2001.

[111] Sugár I.P., Thompson T.E., Biltonen R.L. Monte Carlo Simulation of Two-Component Bilayers: DMPC/DSPC Mixtures. Biophys. J., 76, pp. 2099-2110, 1999.

[112] Tabony J., Perly B. Quasielastic Neutron Scattering Measurements of Fast Local Translational Diffusion of Lipid Molecules in Phospholipid Bilayers. Biochim. Biophys. Acta, 1063, pp. 67-72, 1990.

[113] Talley C.E., Dunn R.C. Single Molecules as Probes of Lipid Membrane Microenvironments. J. Chem. Phys., 103, pp. 10214-10220, 1999. 
[114] Tanford C. Physical Chemistry of Macromolecules. Wiley, New York, 1961.

[115] Tanford C. The Hydrofbic Effect. Wiley, New York, 1973.

[116] Taylor K.M.G., Morris R.M. Thermal Analysis of Phase Transition Behaviour in Liposomes. Theor. Chim. Acta, 248, pp. 289-301, 1995.

[117] Tieleman D.P., Berendsen H.J.C., Sansom M.S.P. Surfice binding of Alamethicin Stabilizes its Helical Structure: Molecular Dynamics Simulations. Biophys. J., 76, pp. 3186-3191, 1999.

[118] Tokumasu F., Jin A.J., Feigenson G.W., Dvorak J.A. Nanoscopic Lipid Domain Dynamics Revealed by Atomic Force Microscopy. Biophys. J., 84, pp. 2609-2618, 2003.

[119] Vaz W., Almeida P. Microscopic Versus Macroscopic Diffusion in One-component Fluid Phase Lipid Bilayer Membranes. Biophys. J., 60, pp. 1553-1554, 1991.

[120] Vaz W., Criado M., Madeira V.M.C., Schoellmann G., Jovin T.M. Size Dependence of the Translational Diffusion of Large Integral Membrane Protein in Liquid-Crystalline Phase Lipid Bilayers. A Study Using Fluorescence Recovery after Photobleaching. Biochemistry, 21, pp. 5608-5612, 1982.

[121] Wahren J., Ekberg K., Johansson J., Henriksson M., Pramanik A., Johansson B.L., Rigler R., Jörnvall H. Role of C-Peptide in Human Physiology. Advanced Materials.

[122] Werma S.P., Wallach D.F.H. Raman Spectroscopy of Lipids and Biolmembranes. In D. Chapman, ed., Topics in Molecular and structural Biology: Biomembrane Structure and Function, pp. 43-166. Verlag Chemie, Weinheim, 1984.

[123] Widengren J. Fluorescence Correlation Spectroscopy, Photophysical Aspects and Applications. Ph.D. thesis, Karolinska Instytut, Stockholm, 1996.

[124] Widengren J. Photophysical Aspects of FCS Measurements. In R. R., E.E. L., eds., Fluorescence Correlation Spectroscopy: Theory and Application, pp. 276-301. Springer, New York, 2001.

[125] Widengren J., Mets U., Rigler R. Fluorescernce Correlation Spectroscopy of Triplet States in Solutions: A Theoretical and Experimental Study. J. Phys. Chem., 99, pp. 13368-13379, 1995. 
[126] Widengren J., Rigler R., Mets U. Triplet State Monitoring by Fluorescernce Correlation Spectroscopy. J. of Fluoresc., 4, pp. 255-258, 1994.

[127] Wiener N. Extrapolation, Interpolation, and Smoothing of Stationary Time Series. The Technology Press of The Massacgusetts Institute of Technology and Wiley, New York, 1950.

[128] Zondervan R., Kulzer F., Orlinskii S.B., Orrit M. Photoblinking of Rhodamine 6G in Poly(vinyl alcohol): Radical Dark State Formed through the Triplet. J. Phys. Chem., 107, pp. 6770-6776, 2003. 


\section{Lebenslauf}

\section{Persönliche Daten}

Name: $\quad$ Agnieszka Hac

Geboren am 22.04.1975 in Zawiercie, Polen

Nationalität: polnisch

Ausbildung

1982-1990

1990-1994

1994-1999

06.1999

$1999-2002$

11.2000-01.2004
Grundschule in Poreba, Polen

Gymnasium "Stefan Zeromski" in Zawiercie, Polen

Studium der Physik in der Fakultät für Physik und Kerntechnik an der Hütten- und Bergakademie, Krakow, Polen.

Fachgebiet: Medizinische Physik und Dosimetrie Magisterarbeit, summa cum laude. Thema: "Water Imaging Phantom of Breast for Clinical Application of the Gamma Probe Modelo-2 in Intraoperative Localization of the Sentinel Node" Leiter: Prof. Marta Wasilewska-Radwanska

Promotionsstudium in der Fakultät für Physik und Kerntechnik an der Hütten- und Bergakademie, Krakow, Polen

Wissenschaftliche Mitarbeiterin an dem Max-Planck-Institut in in Göttingen für biophysikalische Chemie, Arbeitsgruppe:

Biophysik und Thermodynamik von Membranen.

Leiter: Prof. Thomas Heimburg

\section{Berufserfahrung und Praktika}

10.1998-07.2000 Assistentin an der Fakultät für Physik und Kerntechnik an der Hütten-und Bergakademie in Krakow, Polen

05.1998-07.1998 Praktikum an der Universität Torino in Italien im Rahmen Sokratesprogrammes

\section{Auszeichnungen}

07.2000 Der Preis der " Polish Nuclear Society" für die beste Magisterarbeit in der Atomistik

1995-1999 4 Auszeichnungen des Rektors der Hütten- und Bergakademie für sehr gute Studienleistungen 